MÉMOIRES DE LA SMF 91

\title{
GLOBAL SOLUTIONS FOR SMALL NONLINEAR LONG RANGE PERTURBATIONS OF TWO DIMENSIONAL SCHRÖDINGER EQUATIONS
}

\author{
Jean-Marc Delort
}

Société Mathématique de France 2002

Publié avec le concours du Centre National de la Recherche Scientifique 


\section{J.-M. Delort}

Laboratoire Analyse Géométrie et Applications, UMR CNRS 7539, Institut Galilée, Université Paris-Nord, 99, Avenue J.-B. Clément, F-93430 Villetaneuse.

E-mail : delort@math.univ-paris13.fr

2000 Mathematics Subject Classification. - 35Q55, 35S50.

Key words and phrases. - Global existence, Nonlinear Schrödinger equation.

Part of this work has been done while the author was visiting N. Hayashi at the Science University of Tokyo, and during a stay at the Erwin Schrödinger Institute in Vienna in the framework of the START project "Nonlinear Schrödinger and quantum Boltzmann equations" (FWF Y137-TEC) of N.J. Mauser. The author expresses his acknowledgements to both institutions. 


\title{
GLOBAL SOLUTIONS FOR SMALL NONLINEAR LONG RANGE PERTURBATIONS OF TWO DIMENSIONAL SCHRÖDINGER EQUATIONS
}

\author{
Jean-Marc Delort
}

\begin{abstract}
Let $Q_{1}, Q_{2}$ be two quadratic forms, and $u$ a local solution of the two dimensional Schrödinger equation $\left(i \partial_{t}+\Delta\right) u=Q_{1}\left(u, \nabla_{x} u\right)+Q_{2}\left(\bar{u}, \nabla_{x} \bar{u}\right)$. We prove that if $Q_{1}$ and $Q_{2}$ do depend on the derivatives of $u$, and if the Cauchy datum is small enough and decaying enough at infinity, the solution exists for all times. The difficulty of the problem originates in the fact that the nonlinear perturbation is a long range one: by this, we mean that it can be written as the product of (a derivative of) $u$ and of a potential whose $L^{\infty}$ space-norm is not time integrable at infinity.
\end{abstract}

Résumé (Solutions globales pour des perturbations nonlinéaires à longue portée de l'équation de Schrödinger en dimension 2)

Soient $Q_{1}, Q_{2}$ deux formes quadratiques et $u$ solution locale de l'équation de Schrödinger en dimension 2 d'espace $\left(i \partial_{t}+\Delta\right) u=Q_{1}\left(u, \nabla_{x} u\right)+Q_{2}\left(\bar{u}, \nabla_{x} \bar{u}\right)$. Nous prouvons que si $Q_{1}$ et $Q_{2}$ dépendent effectivement des dérivées de $u$, et si la donnée de Cauchy est assez petite et assez décroissante à l'infini, la solution existe globalement en temps. La difficulté du problème réside dans le fait que la perturbation nonlinéaire est à longue portée, en ce sens qu'elle s'écrit comme un produit (d'une dérivée) de $u$ par un potentiel dont la norme $L^{\infty}$ en espace n'est pas intégrable lorsque $t \rightarrow+\infty$. 



\section{CONTENTS}

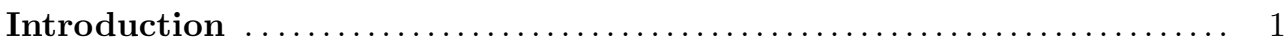

1. The nonlinear Schrödinger equation $\ldots \ldots \ldots \ldots \ldots \ldots \ldots \ldots \ldots \ldots \ldots$

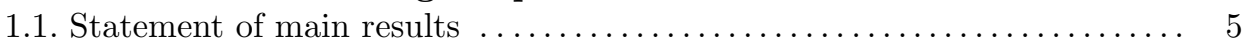

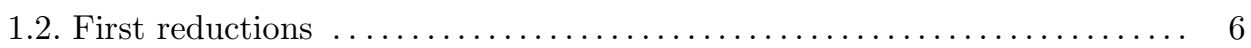

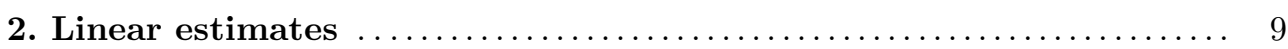

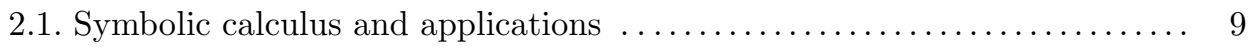

2.2. Spaces of distributions and linear inequality $\ldots \ldots \ldots \ldots \ldots \ldots \ldots \ldots \ldots$

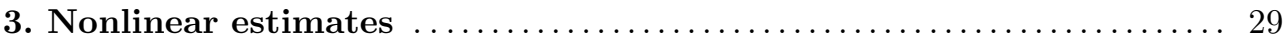

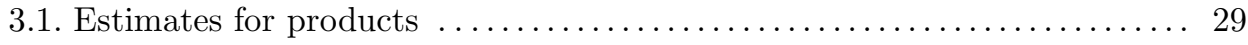

3.2. Conjugation by an oscillatory exponential $\ldots \ldots \ldots \ldots \ldots \ldots \ldots \ldots \ldots$

4. Proof of the main theorem $\ldots \ldots \ldots \ldots \ldots \ldots \ldots \ldots \ldots \ldots \ldots \ldots \ldots \ldots \ldots \ldots \ldots \ldots \ldots \ldots$

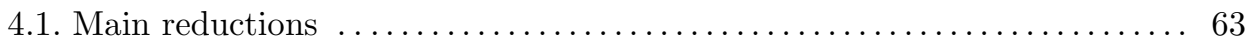

4.2. Existence of the global solution $\ldots \ldots \ldots \ldots \ldots \ldots \ldots \ldots \ldots \ldots \ldots$

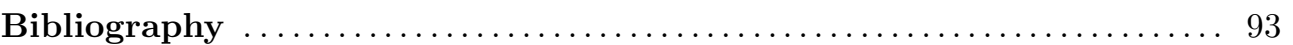





\section{INTRODUCTION}

This paper is devoted to the proof of existence of global solutions for a nonlinear Schrödinger equation in two space dimension with small Cauchy data. Consider the equation

$$
\begin{aligned}
\left(i \partial_{t}+\Delta\right) u & =F\left(u, \nabla_{x} u, \bar{u}, \nabla_{x} \bar{u}\right) \\
\left.u\right|_{t=0} & =\varepsilon u_{0}
\end{aligned}
$$

where $t \in \mathbb{R}, x \in \mathbb{R}^{d}, F$ is a polynomial vanishing at least at order 2 at 0 and $\varepsilon>0$.

The problem of local existence for the above equation with a general nonlinearity and for small Cauchy data (i.e. small $\varepsilon$ ) in a convenient Sobolev space has been solved by Kenig, Ponce and Vega [19]. Hayashi and Ozawa [18] obtained local existence in one space dimension for large Cauchy data. The case of any space dimension was treated by Chihara [4]. More recently, Kenig, Ponce and Vega [20] proved the similar result for a generalized Schrödinger equation, i.e. an equation in which $\Delta$ is replaced by a more general operator.

We are interested in this paper in global solutions for small enough $\varepsilon$. When the space dimension is larger or equal to 3 , and $F$ vanishes at least at order 3 at 0 , Chihara [5], [6] proved that there is a global solution if the data are small enough in a (weighted) Sobolev space. He also proved the same result in two space dimensions under a convenient restriction on the cubic part of the nonlinearity.

For quadratic nonlinearities, and space dimension larger or equal to three, global existence for small data has been obtained under convenient assumptions on the nonlinearity. The most recent results are due to Hayashi and Hirata [10], Hayashi and Kato [11], Hayashi, Miao and Naumkin [12]. We refer the reader to the introduction of [15] for a detailed discussion of these results as well as further references.

The results we have mentioned so far could be qualified of "short range" type ones. By this, we mean the following: the nonlinearity $F$ can be written as a sum of products of a nonlinear potential $V\left(u, \nabla_{x} u, \bar{u}, \nabla_{x} \bar{u}\right)$ times $u$ or $\nabla_{x} u$ or $\bar{u}$ or $\nabla_{x} \bar{u}$. 
Denote by $k \geqslant 1$ the order of vanishing of $V$ at the origin. Since linear solutions of the Schrödinger operator decay in $L^{\infty}$ like $t^{-d / 2}$ when $t \rightarrow+\infty$, we see that $V$ computed on such a solution decays like $t^{-k d / 2}$ when $t \rightarrow+\infty$. We say that $F$ is a short range perturbation of the linear Schrödinger equation if this quantity is integrable when $t \rightarrow+\infty$ i.e. if $k d / 2>1$. All the results we have indicated above fall into this category.

We are interested in this paper in the long range case, more precisely in the limiting case $k d / 2=1$. There are only two such possibilities: either the space dimension $d$ is 1 and $F$ is cubic, or $d=2$ and $F$ is quadratic. The former case has been solved in general by Hayashi and Naumkin [14]: they found a sufficient condition on the cubic nonlinearity under which solutions are global for small enough Cauchy data in a weighted Sobolev space. Their method relies on the use of the smoothing property of Doi [9]. They could also in [17] reduce a particular quadratic nonlinearity to a cubic one, thus obtaining global existence in this case as well.

The case of quadratic nonlinearities in two space dimensions is studied by Cohn [7] for a very specific nonlinearity, and by Hayashi and Naumkin [15], [16] in the special case of real analytic Cauchy data. Such an assumption allows one to avoid the difficulty of the loss of one derivative in the right hand side of the equation.

Our aim in this paper is to study this quadratic two dimensional problem when the Cauchy datum lies in a weighted Sobolev space. We are thus obliged to cope with the problem of recovering the derivative lost in the right hand side. We state our main theorem of global existence in the first chapter, together with precise assumption on the quadratic nonlinearity we consider. Let us just describe here our general strategy in the special case

$$
\left(D_{t}+D_{x}^{2}\right) u=u\left(D_{x_{1}} u\right)
$$

where $D_{t}=\frac{1}{i} \partial_{t}, D_{x_{j}}=\frac{1}{i} \partial_{x_{j}}, j=1,2$, and where the datum is given at $t=1$, the solution being looked for on $\{t \geqslant 1\}$. We first take new coordinates $T=t, X=x / t$ and look for $u$ in terms of a new unknown $w(T, X)$ through $u(t, x)=\frac{1}{t} e^{i x^{2} / 4 t} w(t, x / t)$. We get for $w$ an equation of form

$$
\left(D_{T}+\frac{D_{X}^{2}}{T^{2}}\right) w=\frac{1}{T} e^{i \theta(T, X)} w\left(\frac{D_{X_{1}}}{T}+\frac{X_{1}}{2}\right) w
$$

where $\theta=T X^{2} / 4$. Let us take a simplified model forgetting the $X_{1} / 2$ term above:

$$
\left(D_{T}+\frac{D_{X}^{2}}{T^{2}}\right) w=\frac{1}{T} e^{i \theta(T, X)} w\left(\frac{D_{X_{1}}}{T} w\right) .
$$

Remark that $D_{X_{j}}$ is the translation in the new coordinates of the operator $t D_{x_{j}}-x_{j} / 2$, which is of constant use in the study of global problems for nonlinear Schrödinger equations with small Cauchy data. Consequently, smoothness relatively to $D_{X}$ will play an essential role. The form of the right hand side of $(0.2)$ shows immediately what are the difficulties we will encounter. First of all, we have a loss of a $D_{X} / T$ derivative in the nonlinearity. To remove this problem, we shall use the Kato local smoothing 
property in the version due to Kenig, Ponce and Vega [20], adapted to our long time framework. Secondly, the right hand side of the equation contains the oscillating factor $e^{i \theta}$, which cannot have any $D_{X}$-smoothness uniformly as time $T \rightarrow+\infty$. To treat these oscillating contributions, we introduce spaces of the following type

$$
\left\{v \in L^{2} ;\left(D_{X} / \sqrt{T}\right)^{s}\left(D_{X} / T\right)^{s^{\prime}} v \in L^{2}\right\}
$$

where $s$ and $s^{\prime}$ are integers. The smoothness relatively to $D_{X} / T$ corresponds to what is gained by the local smoothing property - and to what is lost in the nonlinearity $w \frac{D_{X_{1}}}{T} w$. The smoothness relatively to $D_{X} / \sqrt{T}$ should be understood as a weak version of smoothness relatively to $t D_{x}-x / 2$ for $u(t, x)$. This type of derivative is natural for the problem because of the form of $\theta(T, X)=(\sqrt{T} X)^{2} / 4$. To study products of elements in (0.3), we will need to have $s$ and $s^{\prime}$ large enough. There will be no problem to ensure that for $s^{\prime}$, but as $\frac{D_{X}}{\sqrt{T}} e^{i \theta}=\frac{\sqrt{T} X}{2} e^{i \theta}$ we cannot expect the right hand side of $(0.2)$ to be in a space of type (0.3) with a positive $s$. Consequently, instead of trying to find directly $w$ in a space of type (0.3), we shall look for $w$ as an expansion $w=v+V_{1}(v) e^{i \theta}$ where $v$ and $V_{1}(v)$ will be essentially in a space (0.3) with large enough $s, s^{\prime}$, and where $V_{1}(v)$ will moreover decay like $(\sqrt{T}|X|)^{-2}$ when $\sqrt{T}|X| \rightarrow+\infty$. When plugging such an expression in $e^{i \theta(T, X)} w\left(\frac{D_{X_{1}}}{T} w\right)$, one gets a first contribution of form $e^{i \theta(T, X)} v\left(\frac{D_{X_{1}}}{T} v\right)$, and remainders decaying like $\langle\sqrt{T} X\rangle^{-2}$. One will choose $V_{1}(v)$ as a function of $v$ such that $\left(D_{T}+\frac{D_{X}^{2}}{T^{2}}\right)\left(V_{1} e^{i \theta}\right)$ equals $\frac{1}{T} v\left(\frac{D_{X_{1}}}{T} v\right) e^{i \theta}$ modulo remainders. This is possible because $\theta$ is a non characteristic phase for the operator $D_{T}+\frac{D_{X}^{2}}{T^{2}}$. In that way, one gets an equation

$$
\left(D_{T}+\frac{D_{X}^{2}}{T^{2}}\right) v=\frac{1}{T} R
$$

where $R$ will be a combination of terms $e^{i m \theta}$ with coefficients decaying at least like $\langle\sqrt{T} X\rangle^{-2}$. Since $\langle\sqrt{T} X\rangle^{-2} e^{i m \theta}$ has some smoothness relatively to $D_{X} / \sqrt{T}$ uniformly in $T$ (actually, this expression accepts two $D_{X} / \sqrt{T}$ derivatives), this shows that we have gained some smoothness in comparison with the right hand side of $(0.2)$. Actually one has to repeat such a method once again, to reduce the equation to $(0.4)$ with a right hand side $R=R\left(v, \frac{D_{X}}{T} v\right)$ with values in a space of type (0.3) with $s \sim 4$. This last equation can then be solved globally using the local smoothing property as mentioned before.

Our paper is organized as follows. In the first chapter, we state our main theorem and perform first reductions. The second chapter is devoted to the proof of the local smoothing inequality that will be essential in the rest of the article. We make use of Littlewood-Paley decompositions to define convenient Sobolev spaces, and to prove the smoothing inequality as a consequence of propagation of singularities. Section 3 is devoted to nonlinear estimates. We make extensive use of the ideas of paradifferential calculus of Bony [1] to study nonlinear operators acting on the Sobolev spaces defined in chapter 2. We also prove results concerning products or conjugation of an element 
of such a Sobolev space with an oscillatory exponential. Section 4 gives the proof of the theorem. We perform the method of elimination of oscillatory exponentials outlined above. The main tool is again paradifferential calculus, which allows us to decompose the right hand side of the equation as a sum of a nice term, and of a really oscillating contribution, that we eliminate using the non charactericity of the phase. Since the true equation is (0.1) rather than $(0.2)$, we use weighted versions of the Sobolev spaces defined in chapter 2 to treat the contribution coming from $X$ in the right hand side of (0.1). When all oscillatory contributions have been cancelled, the proof of the theorem, as well as the description of the asymptotics of the solution, follow from standard arguments. 
CHAPTER 1

\section{THE NONLINEAR SCHRÖDINGER EQUATION}

\subsection{Statement of main results}

Denote by $(t, x)=\left(t, x_{1}, x_{2}\right)$ coordinates on $\mathbb{R} \times \mathbb{R}^{2}, D_{t}=\frac{1}{i} \frac{\partial}{\partial t}, D_{x_{j}}=\frac{1}{i} \frac{\partial}{\partial x_{j}}$, $D_{x}=\left(D_{x_{1}}, D_{x_{2}}\right)$. Let us denote by $Q_{1}\left(X_{0}, X_{1}, X_{2}\right), Q_{2}\left(X_{0}, X_{1}, X_{2}\right)$ two polynomials in three indeterminates, with complex coefficients, homogeneous of degree 2 , satisfying the following assumption:

$$
Q_{1} \text { and } Q_{2} \text { vanish identically along }\left(X_{1}, X_{2}\right)=(0,0) .
$$

We will be interested in solutions to the following Schrödinger equation

$$
\left(D_{t}+D_{x}^{2}\right) u=Q_{1}\left(u, D_{x} u\right)+Q_{2}\left(\bar{u}, \overline{D_{x} u}\right) .
$$

We want to prove global existence for small Cauchy data. Since (1.1.2) is invariant under translations relatively to the time variable, we can without any loss of generality take Cauchy data at $t=1$ instead of $t=0$, and study solutions for $t \geqslant 1$. Our initial datum will have to be smooth enough and will have to decay rapidly enough at infinity. We thus introduce for $M \in \mathbb{N}$

$$
\begin{aligned}
\mathcal{H}^{M}\left(\mathbb{R}^{2}\right) & =\left\{u \in L^{2}\left(\mathbb{R}^{2}\right) ; \forall \alpha, \beta \in \mathbb{N}^{2},|\alpha|+|\beta| \leqslant M, x^{\alpha} \partial_{x}^{\beta} u \in L^{2}\left(\mathbb{R}^{2}\right)\right\} \\
& =\left\{u \in H^{M}\left(\mathbb{R}^{2}\right) ; \forall \gamma \in \mathbb{N}^{2},|\gamma| \leqslant M, x^{\gamma} u \in H^{M-\gamma}\left(\mathbb{R}^{2}\right)\right\}
\end{aligned}
$$

where $H^{M}\left(\mathbb{R}^{2}\right)$ denotes the usual Sobolev space. Remark that the first characterization of $\mathcal{H}^{M}\left(\mathbb{R}^{2}\right)$ implies that if $\lambda$ is any real number, $\mathcal{H}^{M}\left(\mathbb{R}^{2}\right)$ is invariant under the transformation

$$
u \longmapsto e^{i \lambda x^{2}} u
$$

Let us state our main result:

THEOREM 1.1.1. - There is $M_{0} \in \mathbb{N}$ with the following properties: for any even integer $M \geqslant M_{0}$, there is $\varepsilon_{0}>0$ such that for any $u_{0}$ in the unit ball of $\mathcal{H}^{M+4}\left(\mathbb{R}^{2}\right)$, and any $\varepsilon \in] 0, \varepsilon_{0}\left[\right.$, equation (1.1.2) with Cauchy datum $\left.u\right|_{t=1}=\varepsilon u_{0}$ has a unique global solution $u \in C^{0}\left(\left[1,+\infty\left[, \mathcal{H}^{M}\left(\mathbb{R}^{2}\right)\right)\right.\right.$. 


\section{REMARKS}

- The problem of local existence for equation (1.1.2) has been solved by Kenig, Ponce and Vega [19] for small Cauchy data. Local existence for arbitrary Cauchy data has been proved by Hayashi and Ozawa [18] in one space dimension, by Chihara [4] in higher space dimension, and by Kenig, Ponce and Vega $[\mathbf{2 0}]$ when $\Delta$ is replaced by a more general operator. The uniqueness assertion of our theorem follows from the local uniqueness obtained by these authors (see [20], theorem 1.2). Consequently, we will have to prove only global existence.

- The number $M_{0}$ in the statement of the theorem has to be taken large enough to apply the aforementioned results of uniqueness. For the proof of existence in the large, we will just have to assume $M_{0} \geqslant 6$.

- In the statement of the theorem, there is apparently a loss of smoothness between the assumptions made on the Cauchy data $-u_{0} \in \mathcal{H}^{M+4}\left(\mathbb{R}^{2}\right)$ - and the conclusion saying that at time $t$ the solution $u(t, \cdot)$ belongs to $\mathcal{H}^{M}\left(\mathbb{R}^{2}\right)$. This comes from the fact that the above spaces are not optimal to solve our problem. An optimal statement, without any artificial loss of smoothness, will be given in theorem 4.2.1 after several reductions and the definition of convenient spaces.

The solution of theorem 1.1.1 has the same asymptotic behaviour as linear solutions.

THEOREM 1.1.2. - Under the assumptions of theorem 1.1.1 there is a $L^{\infty}$ function $v_{\infty}(x)$, satisfying $(1+|x|)^{M} v_{\infty}(x) \in L^{\infty}$, and such that for any $\left.\delta \in\right] 0,1 / 2[$ the solution $u$ has the following asymptotic behaviour:

$$
\left\|\left(1+\left|\frac{x}{t}\right|\right)^{M}\left(u(t, x)-\frac{1}{t} e^{i x^{2} / 4 t} v_{\infty}(x / t)\right)\right\|_{L^{\infty}(d x)}=O\left(t^{-1-\delta}\right), t \longrightarrow+\infty .
$$

\subsection{First reductions}

We look for a solution to (1.1.2) in new coordinates

$$
T=t, \quad X=\frac{x}{t}
$$

and take as a new unknown the function $w(T, X)$ related to $u$ through the relation

$$
u(t, x)=\frac{1}{t} e^{i x^{2} / 4 t} w(t, x / t) .
$$

Let us set

$$
\theta(T, X)=\frac{T X^{2}}{4}, \quad Z_{j}=\frac{D_{X_{j}}}{T}+\frac{X_{j}}{2}, \quad j=1,2, Z=\left(Z_{1}, Z_{2}\right) .
$$


Then

$$
\begin{gathered}
D_{x} u(t, x)=\left.\frac{1}{T} e^{i \theta(T, X)}(Z w)(T, X)\right|_{T=t, X=x / t} \\
\left(D_{t}+D_{x}^{2}\right) u(t, x)=\left.\frac{1}{T} e^{i \theta(T, X)}\left[D_{T}+\frac{D_{X}^{2}}{T^{2}}\right] w(T, X)\right|_{T=t, X=x / t} .
\end{gathered}
$$

We deduce from these relations that $u$ is a solution of (1.1.2) if and only if $w$ solves

$$
\left(D_{T}+\frac{D_{X}^{2}}{T^{2}}\right) w=\frac{1}{T} e^{i \theta(T, X)} Q_{1}(w, Z w)+\frac{1}{T} e^{-3 i \theta(T, X)} Q_{2}(\bar{w}, \overline{Z w}) .
$$

Since the transformation $u \mapsto e^{-i x^{2} / 4} u$ leaves $\mathcal{H}^{M+4}\left(\mathbb{R}^{2}\right)$ invariant, as remarked before, the initial condition for (1.2.5) will be

$$
\left.w\right|_{T=1}=\varepsilon w_{0}
$$

with $w_{0}$ given in a fixed ball of $\mathcal{H}^{M+4}\left(\mathbb{R}^{2}\right)$.

Let us indicate what will be our general strategy to find a global solution to (1.2.5) for small Cauchy data. If, in the right hand side of (1.2.5), we had no oscillating factor like $e^{i \theta}, e^{-3 i \theta}$, a proof similar to the one of $[8]$ for global existence for the nonlinear Klein-Gordon equation would work: namely, we could look for a solution $w$ in a Sobolev space of smooth enough functions of $X$, endowed with norms depending conveniently on $T$. One would then have to prove $L^{2}$ inequalities for these Sobolev norms, and to supplement them by an $L^{\infty}$ estimate deduced from the equation. The only new difficulty, in comparison with the Klein-Gordon case, would come from the loss of one derivative in the right hand side, and we would have to compensate for this loss using the smoothing property of the equation.

The oscillating factors $e^{i \theta}, e^{-3 i \theta}$ prevent us from applying directly such a strategy: actually, taking $D_{X}$-derivatives of such exponentials makes appear a $T X$ factor, which has bad behaviour as $T \rightarrow+\infty$. In other words, we cannot expect to put the right hand side of (1.2.5) in any space of smooth functions in $X$ uniformly in $T \rightarrow+\infty$. The idea will be to use the fact that the phases $\theta$ and $-3 \theta$ are non-characteristic for the operator $D_{T}+D_{X}^{2} / T^{2}$. We shall use this property looking for the solution $w$ as $w=v+V_{1} e^{i \theta}+V_{-3} e^{-3 i \theta}$ where $V_{1}, V_{-3}$ will be expressions in terms of the new unknown $v$. These functions $V_{1}, V_{-3}$ will have the same smoothness as $v$, but will enjoy better decay assumptions when $\sqrt{T}|X| \rightarrow+\infty$, and will be chosen to cancel the worst contributions to the right hand side of (1.2.5). In that way, we will reduce (1.2.5) to an equation on $v$, in which the right hand side will no longer contain any harmful oscillating factors, and to which the strategy discussed above can be applied.

REMARK. - Let us comment on the assumptions on the nonlinearity. The fact that we exclude nonlinearities of type $B\left(u, D_{x} u ; \bar{u}, \overline{D_{x} u}\right)$, where $B$ is a bilinear form, comes from the fact that they would induce in the right hand side of (1.2.5) terms in $e^{-i \theta}$, where $-\theta$ is a characteristic phase for $D_{T}+D_{X}^{2} / T^{2}$ when $T \rightarrow+\infty$. Because of that, we could not get rid of these terms by a normal form method as indicated above. We 
believe that this difficulty is not just a technical one: if one tries to find asymptotic solutions through an ansatz inspired by the asymptotics of linear solutions, the same problem appears, preventing one from constructing an asymptotic series as a formal solution.

The second restriction on the nonlinearity coming from (1.1.1), namely the fact that terms like $u^{2}$ or $\bar{u}^{2}$ are excluded, is related to the fact that $\theta$ has a critical point at $X=0$. Because of that $\theta$ is a really oscillating factor only when $\sqrt{T}|X| \rightarrow+\infty$, and the normal form method alluded to above can be carried out only in a region $\sqrt{T}|X| \geqslant 1$. To be able to treat the contribution of the domain $\sqrt{T}|X| \leqslant 1$, we need some extra-vanishing of the non-linearity at $X=0$, which is provided by at least one $Z$-derivative of $w$. We have no conjecture on what happens when (1.1.1) is not satisfied. We remark anyway that in three space dimension Hayashi and Naumkin [13] have been able to get global solutions for nonlinearities of type $u^{2}$ or $\bar{u}^{2}$. 


\section{CHAPTER 2}

\section{LINEAR ESTIMATES}

In the rest of that paper, we will only use the coordinates introduced in (1.2.1). We will thus employ the notation $(t, x)$ instead of $(T, X)$ without any risk of confusion. The aim of this chapter is to introduce, using dyadic decompositions, the spaces of distributions in which we will look for the solutions of equation (1.2.5). The first problem will be to prove a linear estimate in these spaces for solutions of $D_{t}+D_{x}^{2} / t^{2}$, reflecting the Kato smoothing property. We will do so adapting to our long time framework the method used by Kenig, Ponce and Vega [20] in the local in time setting.

\subsection{Symbolic calculus and applications}

The spaces we shall introduce in the following section will be of uniformly local type. Their definition will rely on the use of cut-off functions relatively to the $x$-variable. On the other hand, to be able to prove smoothing estimates in those spaces, we shall have to decompose their elements using pseudo-differential partitions of identity invariant under the hamiltonian flow of the Schrödinger operator. To simplify the study of the interaction of these different localizations, we first establish some general results. We shall use throughout the whole paper the notation $\langle x\rangle=\left(1+x^{2}\right)^{1 / 2}$.

Definition 2.1.1. - A $\left(C_{0}, N_{0}\right)$-temperate weight (for a given positive constant $C_{0}$ and a given $N_{0} \in \mathbb{N}$ ) will be a $C^{\infty}$ function $A: \mathbb{R}^{d} \times \mathbb{R}^{d} \rightarrow \mathbb{R},(x, \xi) \mapsto A(x, \xi)$, satisfying for any $(x, \xi) \in \mathbb{R}^{2 d}$, any $(y, \eta) \in \mathbb{R}^{2 d}$

$$
A(x, \xi) \geqslant 1, \quad \frac{A(x, \xi)}{A(y, \eta)}+\frac{A(y, \eta)}{A(x, \xi)} \leqslant C_{0}(\langle x-y\rangle+\langle\xi-\eta\rangle)^{N_{0}} .
$$

Definition 2.1.2. - Let $A$ be a temperate weight. We shall denote by $S\left(A^{-\infty}\right)$ the space of all smooth functions $(x, \xi) \mapsto a(x, \xi)$, defined on $\mathbb{R}^{d} \times \mathbb{R}^{d}$, with values in $\mathbb{C}$, 
such that for any $N \in \mathbb{N}, n \in \mathbb{N}$

$$
|a|_{A, N, n} \stackrel{\text { def }}{=} \sup _{|\alpha|+|\beta| \leqslant n} \sup _{(x, \xi)}\left|A(x, \xi)^{N} \partial_{x}^{\alpha} \partial_{\xi}^{\beta} a(x, \xi)\right|<+\infty .
$$

We define in the obvious way the notion of a bounded family of symbols.

Remark. - Since $A \geqslant 1$, it follows that $S\left(A^{-\infty}\right) \subset S_{0,0}^{0}$. Consequently, by the Calderón-Vaillancourt theorem [2], any pseudo-differential operator with symbol in $S\left(A^{-\infty}\right)$ is bounded on $L^{2}\left(\mathbb{R}^{d}\right)$, its operator norm being controlled in terms of a semi-norm (2.1.2) of the symbol.

We will need a result of symbolic calculus. If $A$ and $B$ are two $\left(C_{0}, N_{0}\right)$-temperate weights, if $a \in S\left(A^{-\infty}\right), b \in S\left(B^{-\infty}\right)$, we denote by $a \# b$ the symbol of the operator $a(x, D) \circ b(x, D)$, given by the oscillatory integral

$$
a \# b(x, \eta)=\frac{1}{(2 \pi)^{d}} \int e^{-i y \xi} a(x, \eta-\xi) b(x-y, \eta) d y d \xi .
$$

In the same way, the adjoint $a(x, D)^{*}$ of $a(x, D)$ has symbol $a^{*}(x, \eta)$ given by

$$
a^{*}(x, \eta)=\frac{1}{(2 \pi)^{d}} \int e^{-i y \xi} \bar{a}(x-y, \eta-\xi) d y d \xi .
$$

Theorem 2.1.3. - For any $C_{0}>0, N_{0} \in \mathbb{N}, N \in \mathbb{N}, n \in \mathbb{N}$, there are $C_{N, n}^{\prime}>0$ and $n^{\prime} \in \mathbb{N}$ such that the following two statements are true:

(i) For any couple of $\left(C_{0}, N_{0}\right)$-temperate weights $A, B$ and for any $a \in S\left(A^{-\infty}\right)$, $b \in S\left(B^{-\infty}\right)$, one has $a b \in S\left((A B)^{-\infty}\right), a \# b-a b \in S\left((A B)^{-\infty}\right)$ with the following estimates

$$
\begin{aligned}
|a b|_{A B, N, n} & \leqslant C_{N, n}^{\prime}|a|_{A, N, n}|b|_{B, N, n} \\
|a \# b-a b|_{A B, N, n} & \leqslant C_{N, n}^{\prime}\left|\partial_{\xi} a\right|_{A, N, n^{\prime}}\left|\partial_{x} b\right|_{B, N, n^{\prime}} .
\end{aligned}
$$

(ii) For any $\left(C_{0}, N_{0}\right)$-temperate weight $A$ and for any $a \in S\left(A^{-\infty}\right)$, one has $a^{*} \in$ $S\left(A^{-\infty}\right)$ with the estimate

$$
\left|a^{*}-\bar{a}\right|_{A, N, n} \leqslant C_{N, n}^{\prime}\left|\partial_{x} \partial_{\xi} a\right|_{A, N, n^{\prime}}
$$

Proof. - (i) The proof of such a result is quite classical. We just have to pay attention to the fact that $C_{N, n}^{\prime}$ depends on $C_{0}, N_{0}, N, n$ but not on the weights themselves. The first inequality (2.1.5) is clear. To prove the second one we write

$$
\begin{aligned}
a \# b(x, \eta)-a b(x, \eta)= & \frac{1}{(2 \pi)^{d}} \int e^{-i y \xi}(a(x, \eta-\xi)-a(x, \eta)) b(x-y, \eta) d y d \xi \\
& +\frac{1}{(2 \pi)^{d}} \int e^{-i y \xi} a(x, \eta)(b(x-y, \eta)-b(x, \eta)) d y d \xi
\end{aligned}
$$

where both integrals are oscillatory ones. The last contribution is zero, as the coefficient of the exponential does not depend on $\xi$ and vanishes for $y=0$. In the first 
integral, let us write $a(x, \eta-\xi)-a(x, \eta)=-\xi \widetilde{a}(x, \xi, \eta)$ with

$$
\widetilde{a}(x, \xi, \eta)=\int_{0}^{1}\left(\partial_{\eta} a\right)(x, \eta-t \xi) d t
$$

We have if $|\alpha|+|\beta|+|\gamma| \leqslant n$

$$
\begin{aligned}
\left|\partial_{x}^{\alpha} \partial_{\xi}^{\beta} \partial_{\eta}^{\gamma} \widetilde{a}(x, \xi, \eta)\right| & \leqslant\left|\partial_{\eta} a\right|_{A, N, n} \int_{0}^{1} A(x, \eta-t \xi)^{-N} d t \\
& \leqslant\left|\partial_{\eta} a\right|_{A, N, n} A(x, \eta)^{-N} C_{0}^{N}\langle\xi\rangle^{N_{0} N}
\end{aligned}
$$

by (2.1.2), (2.1.1). By integration by parts, the first contribution to the right hand side of $(2.1 .7)$ is

$$
\frac{1}{(2 \pi)^{d}} \int e^{-i y \xi} \widetilde{a}(x, \xi, \eta)\left(D_{x} b\right)(x-y, \eta) d y d \xi .
$$

Let $L_{1}\left(y, D_{\xi}\right)=\langle y\rangle^{-2}\left(1-y \cdot D_{\xi}\right), L_{2}\left(\xi, D_{y}\right)=\langle\xi\rangle^{-2}\left(1-\xi \cdot D_{y}\right)$. Integrating by parts, we get that (2.1.9) equals

$$
\left.\frac{1}{(2 \pi)^{d}} \int e^{-i y \xi(t} L_{1}\left(y, D_{\xi}\right)\right)^{k}\left({ }^{t} L_{2}\left(\xi, D_{y}\right)\right)^{k}\left[\widetilde{a}(x, \xi, \eta)\left(D_{x} b\right)(x-y, \eta)\right] d y d \xi .
$$

Using (2.1.8), and the analogous inequality

$$
\left|\partial_{x}^{\alpha} \partial_{\eta}^{\beta} D_{x} b(x-y, \eta)\right| \leqslant\left|\partial_{x} b\right|_{B, N, n} B(x, \eta)^{-N} C_{0}^{N}\langle y\rangle^{N_{0} N}
$$

for $|\alpha|+|\beta| \leqslant n$, we see that the modulus of (2.1.10) is smaller than

$$
C_{k, N} \int\langle y\rangle^{-k+N_{0} N}\langle\xi\rangle^{-k+N_{0} N} d y d \xi(A(x, \eta) B(x, \eta))^{-N}\left|\partial_{\eta} a\right|_{A, N, k}\left|\partial_{x} b\right|_{B, N, k} .
$$

If $k$ is large enough relatively to $N$, we get the inequality

$$
|a \# b-a b|_{A B, N, 0} \leqslant C_{N, k}\left|\partial_{\eta} a\right|_{A, N, k}\left|\partial_{x} b\right|_{B, N, k}
$$

where $C_{N, k}$ depends on $N, C_{0}, N_{0}$ but not on $A$ nor $B$. The derivatives of $a \# b-a b$ being estimated in the same way, the conclusion follows.

(ii) The proof is similar.

Example. - We will consider in the following families of weights $A_{q, \delta}(x, \xi)$, or $A_{q, \delta}(t, x, \xi)$, depending on parameters $\left.\left.q \in \mathbb{Z}^{d}, \delta \in\right] 0,1\right]$, and eventually $t \in[1,+\infty[$, satisfying (2.1.1) with $C_{0}$ and $N_{0}$ uniform in $t, \delta, q$. We will then consider symbols $a_{q, \delta}(x, \xi)$ or $a_{q, \delta}(t, x, \xi)$ satisfying for any $\alpha, \beta \in \mathbb{N}^{d}$

$$
\left|\partial_{x}^{\alpha} \partial_{\xi}^{\beta} a_{q, \delta}(t, x, \xi)\right| \leqslant C_{\alpha, \beta, N} \delta^{|\alpha|+|\beta|} A_{q}(x, \xi)^{-N}
$$

with $C_{\alpha, \beta, N}$ independent of $q, \delta$.

In other words, we will have for any $\alpha, \beta \in \mathbb{N}^{d}, n \in \mathbb{N}$

$$
\sup _{q, t}\left|\partial_{x}^{\alpha} \partial_{\xi}^{\beta} a_{q, \delta}(t, \cdot, \cdot)\right|_{A_{q, \delta}, N, n}=O\left(\delta^{|\alpha|+|\beta|}\right), \quad \delta \longrightarrow 0 .
$$

This introduction of $\delta$ gives us, according to (2.1.12) and (2.1.5), (2.1.6), a class of symbols in which we do have a symbolic calculus when $\delta \rightarrow 0$. 
As an example, let us take $\phi \in C_{0}^{\infty}(]-1,1\left[^{d}\right), 0 \leqslant \phi \leqslant 1$, such that if we set $\phi_{q}(x)=\phi(x-q)$ we have $\sum_{q \in \mathbb{Z}^{d}} \phi_{q}(x) \equiv 1$. For $\left.\left.\delta \in\right] 0,1\right]$ put $\phi_{q, \delta}(x)=\phi_{q}(\delta x)$. If we set $D_{q, \delta}(x, \xi)=\langle\delta x-q\rangle$, we see that (2.1.1) is satisfied by this weight with a uniform $\left(C_{0}, N_{0}\right)$. Moreover, $\phi_{q, \delta}(x)$ satisfies $(2.1 .12)$ with $A_{q, \delta}$ replaced by $D_{q, \delta}$.

We will need the following proposition:

Proposition 2.1.4. - Let $A_{q, \delta}(t, x, \xi)$ be a family of weights satisfying (2.1.1) with a uniform $\left(C_{0}, N_{0}\right)$. Assume moreover that there is $N_{1} \in \mathbb{N}, C_{1}>0$ with

$$
\sum_{q} A_{q, \delta}(t, x, \xi)^{-N_{1}} \leqslant C_{1}
$$

for any $t \geqslant 1, \delta \in] 0,1],(x, \xi) \in \mathbb{R}^{d} \times \mathbb{R}^{d}$. Let $a_{q, \delta}(t, \cdot) \in S\left(A_{q, \delta}(t, \cdot)^{-\infty}\right)$ satisfying (2.1.12). Assume that there is a positive constant $c$ such that $\sum_{q}\left|a_{q, \delta}(t, x, \xi)\right|^{2} \geqslant c$ for any $t \geqslant 1, \delta \in] 0,1],(x, \xi) \in \mathbb{R}^{d} \times \mathbb{R}^{d}$. Let $r \in[1,+\infty]$. There is $\delta_{0}>0, C>0$ such that for any $\left.\delta \in] 0, \delta_{0}\right]$, any interval $I \subset\left[1,+\infty\left[\right.\right.$, any $u \in L^{2}\left(I \times \mathbb{R}^{d}\right)$ one has the estimate

$$
\begin{aligned}
& C^{-1}\|\| \mathbb{1}_{I}(t) \phi_{p, \delta}(x) u(t, x)\left\|_{L^{2}\left(\frac{d t}{t} d x\right)}\right\|_{\ell_{p}^{r}} \\
& \leqslant\left\|\left(\sum_{q}\left\|\phi_{p, \delta} \mathbb{1}_{I}(t) a_{q, \delta}(t, x, D) u(t, x)\right\|_{L^{2}\left(\frac{d t}{t} d x\right)}^{2}\right)^{1 / 2}\right\|_{\ell_{p}^{r}} \\
& \quad \leqslant C\|\| \mathbb{1}_{I}(t) \phi_{p, \delta}(x) u(t, x)\left\|_{L^{2}\left(\frac{d t}{t} d x\right)}\right\|_{\ell_{p}^{r}}
\end{aligned}
$$

where $\ell_{p}^{r}$ means the $\ell^{r}$ norm relatively to $p$.

If $u \in C^{0}\left(\left[1,+\infty\left[, L^{2}\left(\mathbb{R}^{d}\right)\right)\right.\right.$, one has for any $t$ the inequality

$$
\begin{array}{r}
C^{-1}\|\| \phi_{p, \delta}(x) u(t, x)\left\|_{L^{2}(d x)}\right\|_{\ell_{p}^{r}} \\
\leqslant\left\|\left(\sum_{q}\left\|\phi_{p, \delta} a_{q, \delta}(t, x, D) u(t, x)\right\|_{L^{2}(d x)}^{2}\right)^{1 / 2}\right\|_{\ell_{p}^{r}} \\
\leqslant C\|\| \phi_{p, \delta}(x) u(t, x)\left\|_{L^{2}(d x)}\right\|_{\ell_{p}^{r}}
\end{array}
$$

with a constant $C$ independent of $t$.

The last inequality in (2.1.14), (2.1.15) holds without the assumption $\sum_{q}\left|a_{q, \delta}\right|^{2} \geqslant c$.

Proof. - Let us write $L_{I}^{2}$ for $L^{2}\left(\frac{d t}{t} d x\right)$ over the interval $I$. We have

$$
\sum_{q}\left\|\phi_{p, \delta} a_{q, \delta}(t, x, D) u\right\|_{L_{I}^{2}}^{2}=\int_{I}\left\langle\sum_{q} a_{q, \delta}(t, x, D)^{*} \phi_{p, \delta}^{2} a_{q, \delta}(t, x, D) u, u\right\rangle \frac{d t}{t} .
$$

Let us define

$$
b_{q, \delta}(t, x, \xi)=\left|a_{q, \delta}(t, x, \xi)\right|^{2}, b_{\delta}(t, x, \xi)=\sum_{q} b_{q, \delta}(t, x, \xi) .
$$


Then $b_{q, \delta} \in S\left(A_{q, \delta}^{-\infty}\right)$ and satisfies (2.1.12). Moreover, assumption (2.1.13) implies that $b_{\delta} \in S(1)$ and satisfies $\left|\partial_{x}^{\alpha} \partial_{\xi}^{\beta} b_{\delta}(t, x, \xi)\right| \leqslant C \delta^{|\alpha|+|\beta|}$. By assumption, we also have $b_{\delta} \geqslant c$.

Since $a_{q, \delta} \in S\left(A_{q, \delta}^{-\infty}\right), \phi_{p, \delta} \in S\left(D_{p, \delta}^{-\infty}\right)$ with $D_{p, \delta}=\langle\delta x-p\rangle$, and since these weights satisfy condition (2.1.1) with uniform $\left(C_{0}, N_{0}\right)$, properties (2.1.5) and (2.1.6) imply that

$$
a_{q, \delta}(t, x, D)^{*} \phi_{p, \delta}^{2} a_{q, \delta}(t, x, D)=\phi_{p, \delta} b_{q, \delta}(t, x, D) \phi_{p, \delta}+R_{p, q, \delta}(t, x, D)
$$

where $R_{p, q, \delta}(t, x, \xi) \in S\left(\left(A_{q, \delta} D_{p, \delta}\right)^{-\infty}\right)$. Moreover, since $a_{q, \delta}(t, x, \xi)$ and $\phi_{p, \delta}$ satisfy estimates of type (2.1.12), inequalities (2.1.5), (2.1.6) imply that the semi-norms of $R_{p, q, \delta}$ are $O\left(\delta^{2}\right)$ when $\delta \rightarrow 0$ uniformly in $p, q$. Summing (2.1.18) in $q$ we get

$$
\sum_{q} a_{q, \delta}(t, x, D)^{*} \phi_{p, \delta}^{2} a_{q, \delta}(t, x, D)=\phi_{p, \delta} b_{\delta}(t, x, D) \phi_{p, \delta}+R_{p, \delta}(t, x, D)
$$

where, because of (2.1.13), $R_{p, \delta}(t, x, \xi) \in S\left(D_{p, \delta}^{-\infty}\right)$, the semi-norms of this symbol being $O\left(\delta^{2}\right)$ uniformly in $p$. Define now

$$
C_{\delta}(t, x, \xi)=\left[b_{\delta}(t, x, \xi)-\frac{c}{2}\right]^{1 / 2} .
$$

Since $b_{\delta} \geqslant c$, we get that $C_{\delta}(t, x, \xi) \in S(1)$ with estimates $\left|\partial_{x}^{\alpha} \partial_{\xi}^{\beta} C_{\delta}\right| \leqslant C \delta^{|\alpha|+|\beta|}$. Applying again theorem 2.1.3, we see that

$$
b_{\delta}(t, x, D)=C_{\delta}(t, x, D)^{*} C_{\delta}(t, x, D)+\frac{c}{2} \operatorname{Id}+R_{\delta}(t, x, D)
$$

where $R_{\delta} \in S(1)$, with semi-norms in this space $O\left(\delta^{2}\right), \delta \rightarrow 0$. Plugging into (2.1.19) we get, changing the notation for $R_{p, \delta}$,

$$
\sum_{q} a_{q, \delta}(t, x, D)^{*} \phi_{p, \delta}^{2} a_{q, \delta}(t, x, D)=\phi_{p, \delta} C_{\delta}^{*} C_{\delta} \phi_{p, \delta}+\frac{c}{2} \phi_{p, \delta} \phi_{p, \delta}+R_{p, \delta}(t, x, D)
$$

where $R_{p, \delta}(t, x, \xi) \in S\left(D_{p, \delta}^{-\infty}\right)$, with semi-norms $O\left(\delta^{2}\right)$ uniformly in $p$. The right hand side of (2.1.16) is thus bounded from below by

$$
\frac{c}{2} \int_{I}\left\|\phi_{p, \delta}(x) u(t, x)\right\|_{L^{2}(d x)}^{2} \frac{d t}{t}+\int_{I}\left\langle R_{p, \delta}(t, x, D) u, u\right\rangle \frac{d t}{t}
$$

and from above by

$$
\begin{aligned}
\frac{c}{2} \int_{I}\left\|\phi_{p, \delta}(x) u(t, x)\right\|_{L^{2}(d x)}^{2} \frac{d t}{t}+\int_{I}\left\|C_{\delta} \phi_{p, \delta} u(t, x)\right\|_{L^{2}(d x)}^{2} \frac{d t}{t} \\
+\int_{I}\left\langle R_{p, \delta}(t, x, D) u, u\right\rangle \frac{d t}{t} .
\end{aligned}
$$

Since $C_{\delta}(t, x, D)$ is bounded on $L^{2}$ uniformly in $\delta$, the conclusion will follow from the following lemma. 
LEMMA 2.1.5. - With the above notations,

$$
\left\|\left|\int_{I}\left\langle R_{p, \delta}(t, x, D) u, u\right\rangle \frac{d t}{t}\right|^{1 / 2}\right\|_{\ell_{p}^{r}} \leqslant C \delta\|\| \mathbb{1}_{I}(t) \phi_{p, \delta} u\left\|_{L^{2}\left(\frac{d t}{t} d x\right)}\right\|_{\ell_{p}^{r}}
$$

for a uniform constant $C>0$.

Proof. - Write

$$
\int_{I}\left\langle R_{p, \delta}(t, x, D) u, u\right\rangle \frac{d t}{t}=\sum_{\ell} \sum_{\ell^{\prime}} \int_{I}\left\langle\widetilde{\phi}_{\ell^{\prime}, \delta}(x) R_{p, \delta} \widetilde{\phi}_{\ell, \delta}\left(\phi_{\ell, \delta} u\right), \phi_{\ell^{\prime}, \delta} u\right\rangle \frac{d t}{t}
$$

where $\widetilde{\phi}_{\ell, \delta}=\widetilde{\phi}(\delta x-\ell)$ for a function $\widetilde{\phi} \in C_{0}^{\infty}\left(\mathbb{R}^{d}\right), \widetilde{\phi} \equiv 1$ close to Supp $\phi$. Since $R_{p, \delta}(t, x, \xi) \in S\left(D_{p, \delta}^{-\infty}\right), \widetilde{\phi}_{\ell, \delta} \in S\left(D_{\ell, \delta}^{-\infty}\right)$, we deduce from theorem 2.1.3 that the symbol $R_{p, \delta}^{\ell, \ell^{\prime}}(t, x, \xi)$ of the operator $\widetilde{\phi}_{\ell^{\prime}, \delta} \circ R_{p, \delta}(t, x, D) \circ \widetilde{\phi}_{\ell, \delta} \operatorname{lies}$ in $S\left(\left(D_{\ell, \delta} D_{p, \delta} D_{\ell^{\prime}, \delta}\right)^{-\infty}\right)$. Moreover, its semi-norms are $O\left(\delta^{2}\right)$ when $\delta \rightarrow 0$ uniformly in $p, \ell, \ell^{\prime}$ since the same is true for $R_{p, \delta}(t, x, \xi)$ i.e. we have

$$
\left|\partial_{x}^{\alpha} \partial_{\xi}^{\beta} R_{p, \delta}^{\ell, \ell^{\prime}}(t, x, \xi)\right| \leqslant C_{\alpha, \beta, N} \delta^{2}\langle\delta x-\ell\rangle^{-N}\left\langle\delta x-\ell^{\prime}\right\rangle^{-N}\langle\delta x-p\rangle^{-N}
$$

with a constant independent of $\delta$ and $p, \ell, \ell^{\prime}$. We deduce from that the uniform estimates

$$
\left|\partial_{x}^{\alpha} \partial_{\xi}^{\beta} R_{p, \delta}^{\ell, \ell^{\prime}}(t, x, \xi)\right| \leqslant C_{\alpha, \beta, N} \delta^{2}\langle\ell-p\rangle^{-N}\left\langle\ell^{\prime}-p\right\rangle^{-N}
$$

whence a similar estimate for the $\mathcal{L}\left(L^{2}\right)$ operator norm of $R_{p, \delta}(t, x, D)$. We deduce from $(2.1 .25)$

$$
\left|\int_{I}\left\langle R_{p, \delta}(t, x, D) u, u\right\rangle \frac{d t}{t}\right|^{1 / 2} \leqslant C \delta \sum_{\ell}\langle p-\ell\rangle^{-N}\left\|\mathbb{1}_{I}(t) \phi_{\ell, \delta} u\right\|_{L^{2}\left(\frac{d t}{t} d x\right)} .
$$

The conclusion of the lemma follows from this inequality.

\subsection{Spaces of distributions and linear inequality}

Let us introduce the dyadic decompositions that will be used in the rest of that paper. Following for instance $[\mathbf{3}]$ chapter 2 , we choose $\varphi \in C_{0}^{\infty}\left(\mathbb{R}^{d}-\{0\}\right), \varphi \geqslant 0$, $\operatorname{Supp} \varphi \subset\{\xi ; 3 / 4<|\xi|<8 / 3\}$ and $\chi \in C_{0}^{\infty}(\{\xi ;|\xi|<4 / 3\}), \chi \geqslant 0, \chi \equiv 1$ close to 0 such that

$$
\begin{gathered}
\chi(\xi)+\sum_{0}^{\infty} \varphi\left(2^{-j} \xi\right) \equiv 1 \\
\left|j-j^{\prime}\right| \geqslant 2 \Longrightarrow \operatorname{Supp} \varphi\left(2^{-j} \cdot\right) \cap \operatorname{Supp} \varphi\left(2^{-j^{\prime}} \cdot\right)=\varnothing \\
j \geqslant 1 \Longrightarrow \operatorname{Supp} \chi \cap \operatorname{Supp} \varphi\left(2^{-j} \cdot\right)=\varnothing .
\end{gathered}
$$


We denote by $\Delta_{j}, j \in \mathbb{N}$ (resp. $\Delta_{-1}$ ) the Fourier multiplier with symbol $\varphi\left(2^{-j} \xi\right)$ (resp. $\chi(\xi))$ so that $\sum_{j=-1}^{+\infty} \Delta_{j}=$ Id. For $j \in \mathbb{N}$, we set

$$
S_{j}=\sum_{k=-1}^{j-1} \Delta_{k}=\chi\left(2^{-j} D\right)
$$

For $\ell \in \mathbb{N}$ set $\Gamma_{\ell}(t)=\mathbb{1}_{\left\{2^{\ell}<t<2^{\ell+1}\right\}}$.

Definition 2.2.1. - Let $s, s^{\prime}, \alpha, \alpha^{\prime}$ be real numbers, $r \in[1,+\infty]$.

(i) One denotes by $F_{\alpha, \alpha^{\prime}, r}^{s, s^{\prime}}$ the space of all distributions $u$ belonging to $L_{\text {loc }}^{2}\left(\left[1,+\infty\left[, \mathcal{S}^{\prime}\left(\mathbb{R}^{d}\right)\right)\right.\right.$ such that there is a constant $C>0$, a sequence $\left(c_{j q}\right)_{j q}$ indexed by $j \geqslant-1, q \in \mathbb{Z}^{d}$, satisfying $\sum_{j}\left(\sum_{q}\left|c_{j q}\right|^{r}\right)^{2 / r} \leqslant 1$, such that

$$
\begin{aligned}
& \left\|\phi_{q}(x) \Delta_{j} u \Gamma_{\ell}(t)\right\|_{L^{2}\left(\frac{d t}{t} d x\right)} \\
& \quad \leqslant C c_{j q} 2^{(j-\ell / 2) \alpha}\left(1+2^{j-\ell / 2}\right)^{-s-\alpha} 2^{(j-\ell) \alpha^{\prime}}\left(1+2^{j-\ell}\right)^{-s^{\prime}-\alpha^{\prime}}
\end{aligned}
$$

for any $j \geqslant-1, q \in \mathbb{Z}^{d}, \ell \in \mathbb{N}$.

(ii) One denotes by $H_{\alpha, \alpha^{\prime}, r}^{s, s^{\prime}}$ the space of all distributions $u$ belonging to $L_{\text {loc }}^{\infty}\left(\left[1,+\infty\left[, \mathcal{S}^{\prime}\left(\mathbb{R}^{d}\right)\right)\right.\right.$ such that there is a constant $C>0$, a sequence $\left(c_{j q}\right)_{j q}$ indexed by $j \geqslant-1, q \in \mathbb{Z}^{d}$, satisfying $\sum_{j}\left(\sum_{q}\left|c_{j q}\right|^{r}\right)^{2 / r} \leqslant 1$, such that

$$
\begin{aligned}
& \left\|\phi_{q}(x) \Delta_{j} u \Gamma_{\ell}(t)\right\|_{L^{\infty}\left(d t, L^{2}(d x)\right)} \\
& \quad \leqslant C c_{j q} 2^{(j-\ell / 2) \alpha}\left(1+2^{j-\ell / 2}\right)^{-s-\alpha} 2^{(j-\ell) \alpha^{\prime}}\left(1+2^{j-\ell}\right)^{-s^{\prime}-\alpha^{\prime}}
\end{aligned}
$$

for any $j \geqslant-1, q \in \mathbb{Z}^{d}, \ell \in \mathbb{N}$.

(iii) One denotes by $\underline{H}_{\alpha, \alpha^{\prime}}^{s, s^{\prime}}$ the space of all distributions $u$ belonging to $L_{\text {loc }}^{\infty}\left(\left[1,+\infty\left[, \mathcal{S}^{\prime}\left(\mathbb{R}^{d}\right)\right)\right.\right.$ such that there is a constant $C>0$, and a sequence $\left(c_{j}\right)_{j}$ in the unit ball of $\ell^{2}$, with

$$
\begin{aligned}
& \left\|\Delta_{j} u \Gamma_{\ell}(t)\right\|_{L^{\infty}\left(d t, L^{2}(d x)\right)} \\
& \quad \leqslant C c_{j} 2^{(j-\ell / 2) \alpha}\left(1+2^{j-\ell / 2}\right)^{-s-\alpha} 2^{(j-\ell) \alpha^{\prime}}\left(1+2^{j-\ell}\right)^{-s^{\prime}-\alpha^{\prime}}
\end{aligned}
$$

for any $j \geqslant-1, \ell \in \mathbb{N}$.

We define the norms in these spaces as the best constant $C>0$.

We will use the notation

$$
\left\|c_{j q}\right\|_{\ell_{j}^{2} \ell_{q}^{r}}=\left(\sum_{j}\left(\sum_{q}\left|c_{j q}\right|^{r}\right)^{2 / r}\right)^{1 / 2}
$$


The definition of the above space is clearer if we write it in the following equivalent way: a distribution $u$ is in $F_{\alpha, \alpha^{\prime}, r}^{s, s^{\prime}}$ if and only if there is a family of $L_{\text {loc }}^{2}([1,+\infty[)-$ functions $\left(c_{j q}(t)\right)_{j q}$ and $C>0$ such that

$$
\begin{aligned}
& \left\|\phi_{q}(x) \Delta_{j} u(t, \cdot)\right\|_{L^{2}(d x)} \\
& \leqslant C c_{j q}(t)\left(\frac{2^{j}}{\sqrt{t}}\right)^{\alpha}\left(1+\frac{2^{j}}{\sqrt{t}}\right)^{-s-\alpha}\left(\frac{2^{j}}{t}\right)^{\alpha^{\prime}}\left(1+\frac{2^{j}}{t}\right)^{-s^{\prime}-\alpha^{\prime}}, \\
& \sup _{\ell}\left\|c_{j q}(t) \Gamma_{\ell}(t)\right\|_{L^{2}(d t / t)} \text { is in the unit ball of } \ell_{j}^{2} \ell_{q}^{r} .
\end{aligned}
$$

One has a similar characterization of $H_{\alpha, \alpha^{\prime}, r}^{s, s^{\prime}}$ replacing in the last condition the $L^{2}(d t / t)$-norm by the $L^{\infty}$ one, and of $\underline{H}_{\alpha, \alpha^{\prime}}^{s, s^{\prime}}$, by

$$
\left\|\Delta_{j} u(t, \cdot)\right\|_{L^{2}(d x)} \leqslant C c_{j}\left(\frac{2^{j}}{\sqrt{t}}\right)^{\alpha}\left(1+\frac{2^{j}}{\sqrt{t}}\right)^{-s-\alpha}\left(\frac{2^{j}}{t}\right)^{\alpha^{\prime}}\left(1+\frac{2^{j}}{t}\right)^{-s^{\prime}-\alpha^{\prime}}
$$

with $\left(c_{j}\right)_{j}$ in the unit ball of $\ell^{2}$. The difference between spaces $\underline{H}_{\alpha, \alpha^{\prime}}^{s, s^{\prime}}$ and $H_{\alpha, \alpha^{\prime}, r}^{s, s^{\prime}}$ is that the latter imposes some $x$-decay when $|x| \rightarrow+\infty$ (for $r<+\infty$ ). Since $\left\|\Delta_{j} u(t, \cdot)\right\|_{L^{2}}^{2} \leqslant C \sum_{q}\left\|\phi_{q} \Delta_{j} u(t, \cdot)\right\|_{L^{2}}^{2}$ we have an injection $H_{\alpha, \alpha^{\prime}, 2}^{s, s^{\prime}} \subset \underline{H}_{\alpha, \alpha^{\prime}}^{s, s^{\prime}}$

The meaning of conditions (2.2.7) is as follows: the weight in the right hand side corresponds to two types of derivatives: a stronger one $D_{x} / \sqrt{t}$ and a weaker one $D_{x} / t$. The indices $s, s^{\prime}$ represent the amount of such derivatives available when $2^{j} / \sqrt{t}$ or $2^{j} / t$ go to infinity. The indices $\alpha, \alpha^{\prime}$ measure the behaviour when $2^{j} / \sqrt{t} \ll 1$ or $2^{j} / t \ll 1$. The weaker derivative $D_{x} / t$ is the one to which the Kato smoothing property will apply, uniformly in $t$, when solving the linear Schrödinger equation. As a last remark, let us say that the above spaces do not depend on the choice of $\varphi, \chi, \phi$.

Let us remark that in definitions $(2.2 .3),(2.2 .4),(2.2 .7)$, we could reverse the orders of $\phi_{q}$ and $\Delta_{j}$ :

Proposition 2.2.2. - A distribution $u$ belongs to $F_{\alpha, \alpha^{\prime}, r}^{s, s^{\prime}}\left(\mathrm{resp} . H_{\alpha, \alpha^{\prime}, r}^{s, s^{\prime}}\right)$ if and only if there is a family of $L_{\mathrm{loc}}^{2}\left(\left[1,+\infty[)\right.\right.$-functions (resp. a family of $L_{\mathrm{loc}}^{\infty}([1,+\infty[)-$ functions) $\left(c_{j q}(t)\right)_{j q}$ and $C>0$ such that

$$
\begin{aligned}
& \left\|\Delta_{j} \phi_{q} u(t, \cdot)\right\|_{L^{2}(d x)} \\
& \leqslant C c_{j q}(t)\left(\frac{2^{j}}{\sqrt{t}}\right)^{\alpha}\left(1+\frac{2^{j}}{\sqrt{t}}\right)^{-s-\alpha}\left(\frac{2^{j}}{t}\right)^{\alpha^{\prime}}\left(1+\frac{2^{j}}{t}\right)^{-s^{\prime}-\alpha^{\prime}}
\end{aligned}
$$

with $\sup _{\ell}\left\|c_{j q}(t) \Gamma_{\ell}(t)\right\|_{L^{2}(d t / t)}\left(\operatorname{resp} . \sup _{\ell}\left\|c_{j q}(t) \Gamma_{\ell}(t)\right\|_{L^{\infty}(d t)}=\left\|c_{j q}\right\|_{L^{\infty}(d t)}\right)$ in the unit ball of $\ell_{j}^{2} \ell_{q}^{r}$.

Proof. - Assume that (2.2.7) is satisfied and let us prove (2.2.8). Choose $\widetilde{\phi} \in$ $C_{0}^{\infty}\left(\mathbb{R}^{d}\right), \widetilde{\phi} \equiv 1$ close to $\operatorname{Supp} \phi, \widetilde{\varphi} \in C_{0}^{\infty}\left(\mathbb{R}^{d}-\{0\}\right), \widetilde{\varphi} \equiv 1$ close to $\operatorname{Supp} \varphi$, 
$\widetilde{\chi} \in C_{0}^{\infty}\left(\mathbb{R}^{d}\right), \widetilde{\chi} \equiv 1$ close to Supp $\chi$, and define $\widetilde{\phi}_{q}(x)=\widetilde{\phi}(x-q), \widetilde{\Delta}_{j}=\widetilde{\varphi}\left(2^{-j} D\right)$, $j \in \mathbb{N}, \widetilde{\Delta}_{-1}=\tilde{\chi}(D)$. Write

$$
\Delta_{j} \phi_{q} u=\sum_{q^{\prime}} \sum_{j^{\prime} \geqslant-1}\left(\Delta_{j} \phi_{q}(x) \widetilde{\Delta}_{j^{\prime}} \widetilde{\phi}_{q^{\prime}}(x)\right)\left(\phi_{q^{\prime}}(x) \Delta_{j^{\prime}} u\right) .
$$

It is enough to show that for any $N \in \mathbb{N}$, there is $C_{N}>0$ with

$$
\left\|\Delta_{j} \phi_{q} \widetilde{\Delta}_{j^{\prime}} \widetilde{\phi}_{q^{\prime}}\right\|_{\mathcal{L}\left(L^{2}\right)} \leqslant C_{N}\left\langle q-q^{\prime}\right\rangle^{-N} 2^{-\left|j-j^{\prime}\right| N}
$$

since the assumption (2.2.7) implies that

$$
\begin{aligned}
& \left\|\phi_{q^{\prime}}(x) \Delta_{j^{\prime}} u(t, x)\right\|_{L^{2}(d x)} \\
& \quad \leqslant C 2^{\left|j-j^{\prime}\right| N_{0}} c_{j^{\prime} q^{\prime}}(t)\left(\frac{2^{j}}{\sqrt{t}}\right)^{\alpha}\left(1+\frac{2^{j}}{\sqrt{t}}\right)^{-s-\alpha}\left(\frac{2^{j}}{t}\right)^{\alpha^{\prime}}\left(1+\frac{2^{j}}{t}\right)^{-s^{\prime}-\alpha^{\prime}}
\end{aligned}
$$

for some large enough $N_{0}$. To prove (2.2.10) we write for any function $w$

$$
\begin{aligned}
\Delta_{j} \phi_{q}(x) & \widetilde{\Delta}_{j^{\prime}} \widetilde{\phi}_{q^{\prime}}(x) w \\
& =\sum_{p} \sum_{p^{\prime}} \sum_{p^{\prime \prime}}\left[\phi_{p}(D) \Delta_{j} \phi_{q}(x) \phi_{p^{\prime}}(D) \widetilde{\Delta}_{j^{\prime}} \widetilde{\phi}_{q^{\prime}}(x) \widetilde{\phi}_{p^{\prime \prime}}(D)\right]\left(\phi_{p^{\prime \prime}}(D) w\right) .
\end{aligned}
$$

Remark that $\phi_{p}(\xi) \varphi\left(2^{-j} \xi\right) \in S\left(D_{p}(\xi)^{-\infty}\right)$ where $D_{p}(\xi)=\langle\xi-p\rangle$, with semi-norms uniformly controlled in $p$. In the same way $\phi_{p^{\prime}}(\xi) \widetilde{\varphi}\left(2^{-j^{\prime}} \xi\right) \in S\left(D_{p^{\prime}}(\xi)^{-\infty}\right), \widetilde{\phi}_{p^{\prime \prime}}(\xi) \in$ $S\left(D_{p^{\prime \prime}}(\xi)^{-\infty}\right), \phi_{q}(x) \in S\left(D_{q}(x)^{-\infty}\right), \widetilde{\phi}_{q^{\prime}}(x) \in S\left(D_{q^{\prime}}(x)^{-\infty}\right)$, with uniform control of semi-norms. By theorem 2.1.3, the operator inside the bracket in the right hand side of (2.2.11) is given by a symbol in $S\left(A_{p, p^{\prime}, p^{\prime \prime}, q, q^{\prime}}(x, \xi)^{-\infty}\right)$, with

$$
A_{p, p^{\prime}, p^{\prime \prime}, q, q^{\prime}}(x, \xi)=D_{p}(\xi) D_{p^{\prime}}(\xi) D_{p^{\prime \prime}}(\xi) D_{q}(x) D_{q^{\prime}}(x),
$$

its semi-norms being controlled uniformly in $p, p^{\prime}, p^{\prime \prime}, q, q^{\prime}$. Since (2.2.12) is bounded from below by $c\left\langle p-p^{\prime}\right\rangle^{1 / 2}\left\langle p-p^{\prime \prime}\right\rangle^{1 / 2}\left\langle q-q^{\prime}\right\rangle$, and since in the summation (2.2.11), $p$ (resp. $p^{\prime}$ ) stays of magnitude $2^{j}$ (resp. $2^{j^{\prime}}$ ), the fact that the $\mathcal{L}\left(L^{2}\right)$-norm of an operator is bounded by the semi-norms of its symbol implies that

$$
\begin{aligned}
& \left\|\Delta_{j} \phi_{q}(x) \widetilde{\Delta}_{j^{\prime}} \widetilde{\phi}_{q^{\prime}}(x) w\right\|_{L^{2}}^{2} \\
& \leqslant C \sum_{p}\left[\sum_{p^{\prime}} \sum_{p^{\prime \prime}} 2^{-N\left|j-j^{\prime}\right|}\left\langle p-p^{\prime}\right\rangle^{-N}\left\langle p-p^{\prime \prime}\right\rangle^{-N}\left\langle q-q^{\prime}\right\rangle^{-N}\left\|\phi_{p^{\prime \prime}}(D) w\right\|_{L^{2}}\right]^{2} \\
& \quad \leqslant C 2^{-2 N\left|j-j^{\prime}\right|}\left\langle q-q^{\prime}\right\rangle^{-2 N}\left\|\sum_{p^{\prime \prime}}\left\langle p-p^{\prime \prime}\right\rangle^{-N}\right\| \phi_{p^{\prime \prime}}(D) w\left\|_{L^{2}}\right\|_{\ell_{p}^{2}}^{2}
\end{aligned}
$$

where we used almost orthogonality in $p$. This inequality implies (2.2.10) and concludes the proof.

The reverse implication $(2.2 .8) \Rightarrow(2.2 .7)$ is shown in a similar way.

Our main objective is to prove the following version of the Kato smoothing property in the framework of the spaces introduced in definition 2.2.1. 
THEOREM 2.2.3. - Let $s, s^{\prime}, \alpha, \alpha^{\prime}$ be real numbers satisfying

$$
\frac{\alpha}{2}+\alpha^{\prime}<0, \quad s+\alpha \geqslant 0, \quad s^{\prime}+\alpha^{\prime} \geqslant 0 .
$$

Take $v_{0} \in H^{s+s^{\prime}}\left(\mathbb{R}^{d}\right), f \in F_{\alpha, \alpha^{\prime}, 1}^{s, s^{\prime}-1 / 2}$. Then the solution $v$ of the linear problem

$$
\begin{aligned}
\left(D_{t}+\frac{D_{x}^{2}}{t^{2}}\right) v & =\frac{1}{t} f \\
\left.v\right|_{t=1} & =v_{0}
\end{aligned}
$$

belongs to $\underline{H}_{\alpha, \alpha^{\prime}}^{s, s^{\prime}} \cap F_{\alpha, \alpha^{\prime}, \infty}^{s, s^{\prime}+1 / 2}$ and we have the smoothing inequality

$$
\|v\|_{\underline{H}_{\alpha, \alpha^{\prime}}^{s, s^{\prime}}}+\|v\|_{F_{\alpha, \alpha^{\prime}, \infty}^{s, s^{\prime}+1 / 2}} \leqslant C\left[\left\|v_{0}\right\|_{H^{s+s^{\prime}}}+\|f\|_{F_{\alpha, \alpha^{\prime}, 1}^{s, s^{\prime}-1 / 2}}\right] .
$$

The main step in the proof will be to obtain estimates for dyadic frequency cut-offs of $v$. For $\delta \in] 0,1], q \in \mathbb{Z}^{d}$, we use the notation $\phi_{q, \delta}(x)=\phi(\delta x-q)$ introduced before proposition 2.1 .4 and $\Gamma_{\ell}(t)=\mathbb{1}_{\left\{2^{\ell}<t<2^{\ell+1}\right\}}$.

THEOREM 2.2.4. - There is $\left.\left.\delta_{0} \in\right] 0,1\right]$ and for any $\left.\left.\delta \in\right] 0, \delta_{0}\right]$ a positive constant $C_{\delta}$ such that for any $v_{0}, f, v$, satisfying the assumptions of theorem 2.2 .3 , any $j \in \mathbb{Z}$, $j \geqslant-1$, any $\ell \in \mathbb{N}$

$$
\begin{aligned}
& \sup _{p}\left\|\Gamma_{\ell}(t) \phi_{p, \delta}(x) \Delta_{j} v\right\|_{L^{2}\left(\frac{d t}{t} d x\right)} \\
& \leqslant C_{\delta}\left(1+2^{j-\ell}\right)^{-1 / 2}\left\|\Delta_{j} v_{0}\right\|_{L^{2}} \\
& \quad+C_{\delta}\left(1+2^{j-\ell}\right)^{-1 / 2} \sum_{m=0}^{\ell}\left(1+2^{j-m}\right)^{-1 / 2}\|\| \Gamma_{m}(t) \phi_{p, \delta} \Delta_{j} f\left\|_{L^{2}\left(\frac{d t}{t} d x\right)}\right\|_{\ell_{p}^{1}} .
\end{aligned}
$$

The proof of this theorem will be done in several steps. Define first

$$
\begin{aligned}
& a_{q, \delta}(t, x, \xi)=\phi_{q, \delta}\left(x+2 \xi\left(\frac{1}{t}-1\right)\right)=\phi\left(\delta\left(x+2 \xi\left(\frac{1}{t}-1\right)\right)-q\right) \\
& A_{q, \delta}(t, x, \xi)=\left\langle\delta\left(x+2 \xi\left(\frac{1}{t}-1\right)\right)-q\right\rangle .
\end{aligned}
$$

We see that $A_{q, \delta}$ is a family of $\left(C_{0}, N_{0}\right)$-temperate weights, with constants $C_{0}, N_{0}$ uniform in $\left.\left.q \in \mathbb{Z}^{d}, \delta \in\right] 0,1\right]$, and that this family satisfies (2.1.13). Moreover $\left.a_{q, \delta}\right|_{t=1}=\phi_{q, \delta}(x)$ and $a_{q, \delta} \in S\left(A_{q, \delta}^{-\infty}\right)$, the semi-norms of these symbols in these spaces being controlled uniformly in $q, \delta$. More precisely, we have for $N \in \mathbb{N}, n \in \mathbb{N}$

$$
\sup _{q}\left|\partial_{x}^{\alpha} \partial_{\xi}^{\beta} a_{q, \delta}\right|_{A_{q, \delta}, N, n}=O\left(\delta^{|\alpha|+|\beta|}\right), \quad \delta \longrightarrow 0 .
$$

The symbols $a_{q, \delta}$ are essentially constant along the bicharacteristics of $D_{t}+D_{x}^{2} / t^{2}$, so that they will enjoy nice commutation properties with this operator. More precisely, denote

$$
b_{q, \delta}(t, x, \xi)=-(\Delta \phi)\left(\delta\left(x+2 \xi\left(\frac{1}{t}-1\right)\right)-q\right) .
$$


We have

$$
\left[D_{t}+\frac{D_{x}^{2}}{t^{2}}, a_{q, \delta}(t, x, D)\right]=\frac{\delta^{2}}{t^{2}} b_{q, \delta}(t, x, D)
$$

whence the equation

$$
\begin{aligned}
\left(D_{t}+\frac{D_{x}^{2}}{t^{2}}\right) a_{q, \delta}(t, x, D) \Delta_{j} v & =\frac{1}{t} a_{q, \delta}(t, x, D) \Delta_{j} f+\frac{\delta^{2}}{t^{2}} b_{q, \delta}(t, x, D) \Delta_{j} v \\
\left.a_{q, \delta}(t, x, D) \Delta_{j} v\right|_{t=1} & =\phi_{q, \delta}(x) \Delta_{j} v_{0} .
\end{aligned}
$$

The first step of the proof is to get an upper bound for the left hand side of $(2.2 .16)$ in terms of $a_{q, \delta}(t, x, D) \Delta_{j} v$ cut-off in convenient time domains.

Proposition 2.2.5. - Choose $\psi \in C_{0}^{\infty}\left(\mathbb{R}^{d}\right), \psi \equiv 1$ on $[-2,2]^{d}, \psi \geqslant 0$, and for $\left.\left.q \in \mathbb{Z}^{d}, \delta \in\right] 0,1\right]$ set $\psi_{q, \delta}(t, \xi)=\psi\left(2 \delta \xi\left(\frac{1}{t}-1\right)-q\right)$. Denote by $\widetilde{\Delta}_{j}$ the dyadic cut-offs introduced at the beginning of the proof of proposition 2.2.2. There $\left.\left.i s \delta_{0} \in\right] 0,1\right]$ and $C>0$ such that for any $\left.\delta \in] 0, \delta_{0}\right]$, any $j \geqslant-1$, any compact interval $I \subset[1,+\infty[$, any $v \in L_{\text {loc }}^{2}\left(\left[1,+\infty\left[, L^{2}(d x)\right)\right.\right.$ one has

$$
\text { (2.2.22) } \sup _{p \in \mathbb{Z}^{d}}\left\|\phi_{p, \delta}(x) \Delta_{j} v(t, x)\right\|_{L_{I}^{2}}^{2} \leqslant C \sup _{p \in \mathbb{Z}^{d}} \sum_{q \in \mathbb{Z}^{d}}\left\|\psi_{q-p, \delta}(t, D) \widetilde{\Delta}_{j} a_{q, \delta}(t, x, D) \Delta_{j} v\right\|_{L_{I}^{2}}^{2}
$$

where $L_{I}^{2}=L^{2}\left(I, \frac{d t}{t} d x\right)$.

To prove this proposition, we shall decompose $\phi_{p, \delta}(x) \Delta_{j} v(t, x)$ as an almost orthogonal sum using $\left(a_{q, \delta}\right)_{q}$. Each term of this decomposition will have microlocal support contained inside $\operatorname{Supp} \phi_{p, \delta}(x) \cap \operatorname{Supp} a_{q, \delta}(t, x, \xi)$, which will imply a $(t, \xi)$ localization, given by $\psi_{q-p, \delta}$ in the right hand side of (2.2.22). This can be visualized on the following picture where, at fixed $\xi$, we represent $u=\frac{1}{t}-1$ as a function of $x$ :

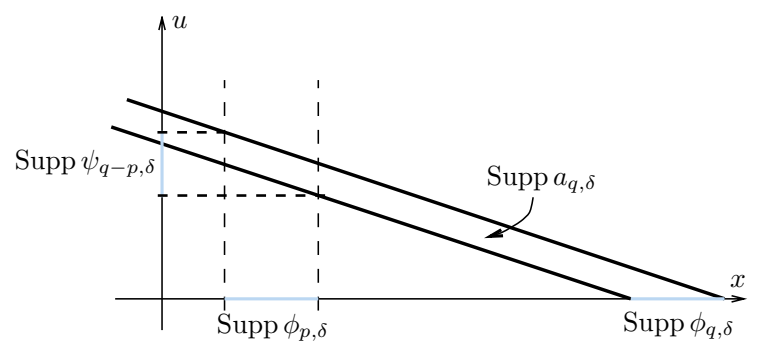

The formal proof will use the following lemma.

Lemma 2.2.6. - Let $A_{q, \delta}(t, x, \xi)$ be a family of temperate weights with uniform constants $\left(C_{0}, N_{0}\right)$, satisfying for some $N_{1} \in \mathbb{N}, C_{1}>0$ the inequality $\sum_{q} A_{q, \delta}(t, x, \xi)^{-N_{1}} \leqslant C_{1}$, uniformly in $t, \delta$. Let $r \in[1,+\infty]$. Denote $D_{q^{\prime}, \delta}(x)=\left\langle\delta x-q^{\prime}\right\rangle$.

Let $R_{q, q^{\prime}, \delta}(t, x, \xi)$ be a family of symbols of $S\left(A_{q, \delta}^{-\infty} D_{q^{\prime}, \delta}(x)^{-\infty}\right)$ with semi-norms in these spaces bounded uniformly in $\delta \in] 0,1], q, q^{\prime} \in \mathbb{Z}^{d}$. There is $C>0$ such that 
for any $w \in L_{\mathrm{loc}}^{2}\left(\left[1,+\infty\left[, L^{2}(d x)\right)\right.\right.$, any compact interval $I$,

$$
\left\|\left(\sum_{q}\left\|R_{q, q^{\prime}, \delta}(t, x, D) w\right\|_{L_{I}^{2}}^{2}\right)^{1 / 2}\right\|_{\ell_{q^{\prime}}^{r}} \leqslant C\|\| \phi_{q^{\prime}, \delta} w\left\|_{L_{I}^{2}}\right\|_{\ell_{q^{\prime}}^{r}} .
$$

If $R_{q, \delta}(x, \xi)$ is a family in $S\left(A_{q, \delta}^{-\infty}\right)$ at fixed $t$, with uniformly bounded semi-norms, there is $C>0$, and for any $w \in L^{2}\left(\mathbb{R}^{2}\right)$

$$
\sum_{q}\left\|R_{q, \delta}(x, D) w\right\|_{L^{2}}^{2} \leqslant C\|w\|_{L^{2}}^{2} .
$$

Proof. - We decompose $w=\sum_{p} \phi_{p, \delta}(x) w$ and write

$$
\begin{aligned}
& \sum_{q}\left\|R_{q, q^{\prime}, \delta}(t, x, D) w\right\|_{L_{I}^{2}}^{2} \\
& \quad=\sum_{p, p^{\prime}, q} \int_{I}\left\langle\left[\widetilde{\phi}_{p^{\prime}, \delta}(x) R_{q, q^{\prime}, \delta}(t, x, D)^{*} R_{q, q^{\prime}, \delta}(t, x, D) \widetilde{\phi}_{p, \delta}\right] \phi_{p, \delta} w, \phi_{p^{\prime}, \delta} w\right\rangle \frac{d t}{t} .
\end{aligned}
$$

By theorem 2.1.3, the operator between brackets $L_{q, q^{\prime}, \delta}^{p, p^{\prime}}(t, x, D)$ has symbol

$$
L_{q, q^{\prime}, \delta}^{p, p^{\prime}}(t, x, \xi) \in S\left(A_{q, \delta}^{-\infty} D_{q^{\prime}, \delta}(x)^{-\infty} D_{p, \delta}(x)^{-\infty} D_{p^{\prime}, \delta}(x)^{-\infty}\right)
$$

with uniformly controlled semi-norms. Since for $N \geqslant N_{1}$,

$$
\begin{aligned}
\sum_{q} A_{q, \delta}(t, x, \xi)^{-N} D_{q^{\prime}, \delta}(x)^{-2 N} D_{p, \delta}(x)^{-N} D_{p^{\prime}, \delta}(x)^{-N} & \\
& \leqslant C_{N}\left\langle q^{\prime}-p\right\rangle^{-N}\left\langle q^{\prime}-p^{\prime}\right\rangle^{-N}
\end{aligned}
$$

$\sum_{q} L_{q, q^{\prime}, \delta}^{p, p^{\prime}}(t, x, \xi)$ is in $S_{0,0}^{0}$ with semi-norms controlled by the right hand side of (2.2.26), uniformly in $\delta$. We deduce from (2.2.25)

$$
\sum_{q}\left\|R_{q, q^{\prime}, \delta}(t, x, D) w\right\|_{L_{I}^{2}}^{2} \leqslant \sum_{p} \sum_{p^{\prime}} C_{N}\left\langle q^{\prime}-p\right\rangle^{-N}\left\langle q^{\prime}-p^{\prime}\right\rangle^{-N}\left\|\phi_{p, \delta} w\right\|_{L_{I}^{2}}\left\|\phi_{p^{\prime}, \delta} w\right\|_{L_{I}^{2}}
$$

from which (2.2.23) follows. One gets (2.2.24) applying the fixed time version of (2.2.23) to $R_{q, q^{\prime}, \delta}(t, x, \xi) \stackrel{\text { def }}{=} \phi_{q^{\prime}, \delta}(x) R_{q, \delta}(x, \xi)$ and $r=2$.

Proof of proposition 2.2.5. - By proposition 2.1.4, for small enough $\delta_{0}$ we have

$$
\sup _{p}\left\|\phi_{p, \delta}(x) \Delta_{j} v(t, x)\right\|_{L_{I}^{2}}^{2} \leqslant C \sup _{p} \sum_{q}\left\|\phi_{p, \delta}(x) a_{q, \delta}(t, x, D) \Delta_{j} v\right\|_{L_{I}^{2}}^{2} .
$$

Remark that if $(t, x, \xi)$ is in the support of $\phi_{p, \delta}(x) a_{q, \delta}(t, x, \xi)$ one has $\delta x-p \in Q$, $\delta\left(x+2 \xi\left(\frac{1}{t}-1\right)\right)-q \in Q$, where $Q$ is the cube $]-1,1\left[{ }^{d}\right.$, whence $\psi_{q-p, \delta}(t, \xi)=1$. We thus write

$$
\phi_{p, \delta}(x) a_{q, \delta}(t, x, D) \Delta_{j}=\phi_{p, \delta}(x) a_{q, \delta}(t, x, D)\left(\widetilde{\Delta}_{j} \psi_{q-p, \delta}(t, D)\right) \Delta_{j} .
$$


The family $a_{q, \delta}(t, x, \xi)$ has semi-norms in $S\left(A_{q, \delta}^{-\infty}\right)$ uniformly bounded (where $A_{q, \delta}$ is defined by (2.2.17)) and moreover, for $N \in \mathbb{N}, n \in \mathbb{N}$

$$
\sup _{q}\left|\partial_{x}^{\alpha} \partial_{\xi}^{\beta} a_{q, \delta}(t, x, \xi)\right|_{A_{q, \delta}, N, n}=O\left(\delta^{|\alpha|+|\beta|}\right), \delta \longrightarrow 0 .
$$

The family $\psi_{q-p, \delta}(t, \xi) \widetilde{\varphi}\left(2^{-j} \xi\right)$ has semi-norms uniformly bounded in the space of symbols $S(1)=S_{0,0}^{0}$. By theorem 2.1.3 we see, taking (2.2.29) into account,

$$
\begin{aligned}
\phi_{p, \delta}(x)\left[a_{q, \delta}(t, x, D)\right]\left[\widetilde{\Delta}_{j} \psi_{q-p, \delta}(t, D)\right] \\
=\phi_{p, \delta}(x)\left[\widetilde{\Delta}_{j} \psi_{q-p, \delta}(t, D)\right]\left[a_{q, \delta}(t, x, D)\right]+\delta R_{q, p, \delta}(t, x, D)
\end{aligned}
$$

where $R_{q, p, \delta}(t, x, \xi)$ is in $S\left(A_{q, \delta}^{-\infty} D_{p, \delta}(x)^{-\infty}\right)$ with uniformly bounded semi-norms. We deduce from (2.2.27) and from lemma 2.2.6 with $r=\infty$

$$
\begin{aligned}
\sup _{p}\left\|\phi_{p, \delta}(x) \Delta_{j} v\right\|_{L_{I}^{2}}^{2} \leqslant C \sup _{p} \sum_{q}\left\|\psi_{q-p, \delta}(t, D) \widetilde{\Delta}_{j} a_{q, \delta}(t, x, D) \Delta_{j} v\right\|_{L_{I}^{2}}^{2} & \\
& +C \delta_{p}^{2} \sup _{p}\left\|\phi_{p, \delta}(x) \Delta_{j} v\right\|_{L_{I}^{2}}^{2} .
\end{aligned}
$$

The conclusion follows taking $\delta$ small enough.

COROLlary 2.2.7. - With the above assumptions and notations, there is $C>0$ such that for any $\left.\delta \in] 0, \delta_{0}\right], \ell \in \mathbb{N}, v \in L_{\mathrm{loc}}^{\infty}\left(\left[1,+\infty\left[, L^{2}\right)\right.\right.$

$$
\begin{aligned}
& \sup _{p}\left\|\Gamma_{\ell}(t) \phi_{p, \delta}(x) \Delta_{j} v(t, x)\right\|_{L^{2}\left(\frac{d t}{t} d x\right)} \\
& \leqslant C \inf \left(1, \frac{2^{(\ell-j) / 2}}{\sqrt{\delta}}\right)\left(\sum_{q}\left\|\sup _{t}\left|\Gamma_{\ell}(t) \mathcal{F}\left(a_{q, \delta}(t, x, D) \Delta_{j} v\right)(t, \xi)\right|\right\|_{L^{2}(d \xi)}^{2}\right)^{1 / 2}
\end{aligned}
$$

where we denoted by $\mathcal{F}$ the Fourier transform in $x$.

Proof. - By proposition 2.2.5, the square of the left hand side is smaller than

$$
C \sup _{p \in \mathbb{Z}^{d}} \sum_{q}\left\|\Gamma_{\ell}(t) \psi_{q-p, \delta}(t, D) \widetilde{\Delta}_{j} a_{q, \delta}(t, x, D) \Delta_{j} v(t, x)\right\|_{L^{2}\left(\frac{d t}{t} d x\right)}^{2} .
$$

The general term of this sum is bounded by Plancherel by

$$
C \int \sup _{t}\left|\Gamma_{\ell}(t) \mathcal{F}\left(a_{q, \delta}(t, x, D) \Delta_{j} v\right)(t, \xi)\right|^{2} d \xi \times \int \Gamma_{\ell}(t) \frac{d t}{t} .
$$

This gives $(2.2 .30)$ when $1 \leqslant 2^{(\ell-j) / 2} / \sqrt{\delta}$. In the other case, we can always assume $j \geqslant 0$. Using that on the support of $\psi_{q-p, \delta}(t, \xi)$ we have the inequality

$$
\left|2 \delta \xi\left(\frac{1}{t}-1\right)-(q-p)\right| \leqslant K,
$$


we estimate the general term of (2.2.31) by

$$
\begin{array}{r}
\int\left|\Gamma_{\ell}(t) \psi_{q-p, \delta}(t, \xi) \widetilde{\varphi}\left(2^{-j} \xi\right) \mathcal{F}\left(a_{q, \delta}(t, x, D) \Delta_{j} v\right)(t, \xi)\right|^{2} d \xi \frac{d t}{t} \\
\leqslant C 2^{\ell} \int \mathbb{1}_{\{|2 \delta \xi(u-1)-(q-p)| \leqslant K\}} \Gamma_{\ell}(1 / u) \\
\quad \times\left.\widetilde{\varphi}\left(2^{-j} \xi\right) \mathcal{F}\left(a_{q, \delta}(1 / u, x, D) \Delta_{j} v\right)(1 / u, \xi)\right|^{2} d \xi d u \\
\leqslant \\
\quad C \frac{2^{\ell-j}}{\delta} \int \sup _{t}\left|\Gamma_{\ell}(t) \mathcal{F}\left(a_{q, \delta}(t, x, D) \Delta_{j} v\right)(t, \xi)\right|^{2} d \xi
\end{array}
$$

where the last inequality follows from the fact that $u$ stays in an interval of length $K / \delta|\xi|$, with $K$ independent of $p, q, \delta$, and $|\xi| \sim 2^{j}$. This concludes the proof.

The factor $2^{(\ell-j) / 2}$ in the right hand side of (2.2.30), represents the gain coming from the smoothing property of the operator. The next step is to estimate the right hand side of (2.2.30) using the equation. To simplify notations let us set

$$
\begin{aligned}
& v_{j q}=a_{q, \delta}(t, x, D) \Delta_{j} v, f_{j q}=a_{q, \delta}(t, x, D) \Delta_{j} f \\
& v_{j q}^{0}=\phi_{q, \delta} \Delta_{j} v_{0}, r_{j q}=b_{q, \delta}(t, x, D) \Delta_{j} v .
\end{aligned}
$$

Proposition 2.2.8. - There are $\left.\left.\delta_{0} \in\right] 0,1\right], C>0$ such that for any $\left.\left.\delta \in\right] 0, \delta_{0}\right]$, any $j \geqslant-1, \ell \in \mathbb{N}, v \in L_{\mathrm{loc}}^{\infty}\left(\left[1,+\infty\left[, L^{2}\right)\right.\right.$ one has

$$
\begin{aligned}
& \|\| \sup _{t} \mid \Gamma_{\ell}(t) \widehat{v}_{j q}(t, \xi)\|\|_{L^{2}(d \xi)} \|_{\ell_{q}^{2}} \\
& \quad \leqslant C\left[\|\| \widehat{v}_{j q}^{0}(\xi)\left\|_{L^{2}(d \xi)}\right\|_{\ell_{q}^{2}}+\|\| \sum_{m=0}^{\ell} \int \Gamma_{m}(t)\left|\widehat{f}_{j q}(t, \xi)\right| \frac{d t}{t}\left\|_{L^{2}(d \xi)}\right\|_{\ell_{q}^{2}}\right] .
\end{aligned}
$$

Proof. - Equation (2.2.21) may be written

$$
\begin{aligned}
\left(D_{t}+\frac{\xi^{2}}{t^{2}}\right) \widehat{v}_{j q}(t, \xi) & =\frac{1}{t} \widehat{f}_{j q}(t, \xi)+\frac{\delta^{2}}{t^{2}} \widehat{r}_{j q}(t, \xi) \\
\widehat{v}_{j q}(1, \xi) & =\widehat{v}_{j q}^{0}(\xi)
\end{aligned}
$$

whence

$$
\begin{aligned}
\widehat{v}_{j q}(t, \xi)=\exp \left(i \frac{\xi^{2}}{t}-i \xi^{2}\right) \widehat{v}_{j q}^{0}(\xi) & \\
& +i \int_{1}^{t} \exp \left(i \xi^{2}\left(\frac{1}{t}-\frac{1}{s}\right)\right)\left[\frac{1}{s} \widehat{f}_{j q}(s, \xi)+\frac{\delta^{2}}{s^{2}} \widehat{r}_{j q}(s, \xi)\right] d s
\end{aligned}
$$

which implies for any $\xi$, any $\ell \in \mathbb{N}$,

$\sup _{t}\left|\Gamma_{\ell}(t) \widehat{v}_{j q}(t, \xi)\right| \leqslant\left|\widehat{v}_{j q}^{0}(\xi)\right|+\sum_{m=0}^{\ell} \int \Gamma_{m}(s)\left|\widehat{f}_{j q}(s, \xi)\right| \frac{d s}{s}+\delta^{2} \sum_{m=0}^{\ell} \int \Gamma_{m}(s)\left|\widehat{r}_{j q}(s, \xi)\right| \frac{d s}{s^{2}}$. 
Taking the $L^{2}(d \xi)$ norm and the $\ell_{q}^{2}$ norm, and setting

$$
\begin{aligned}
\alpha_{\ell j} & =\|\| \sup _{t}\left|\Gamma_{\ell}(t) \widehat{v}_{j q}(t, \xi)\right|\|\|_{L^{2}(d \xi)} \|_{\ell_{q}^{2}} \\
a_{\ell j} & =\|\| \widehat{v}_{j q}^{0}\left\|_{L^{2}(d \xi)}\right\|_{\ell_{q}^{2}}+\|\| \sum_{m \leqslant \ell} \int \Gamma_{m}(s)\left|\widehat{f}_{j q}(s, \xi)\right| \frac{d s}{s}\|\|_{L^{2}(d \xi)} \|_{\ell_{q}^{2}} \\
\beta_{j}(s) & =\|\| \widehat{r}_{j q}(s, \xi)\left\|_{L^{2}(d \xi)}\right\|_{\ell_{q}^{2}}
\end{aligned}
$$

we get the inequality

$$
\alpha_{\ell j} \leqslant a_{\ell j}+\delta^{2} \sum_{m=0}^{\ell} \int \Gamma_{m}(s) \beta_{j}(s) \frac{d s}{s^{2}} .
$$

Applying the second and the first inequalities (2.1.15) with $r=2$, we have by definition of $r_{j q}, v_{j q}$

$$
\beta_{j}(s) \leqslant C\left\|\Delta_{j} v(s, \cdot)\right\|_{L^{2}} \leqslant C\|\| \widehat{v}_{j q}(s, \xi) \mid\left\|_{L^{2}(d \xi)}\right\|_{\ell_{q}^{2}}
$$

with a constant $C$ independent of $s$. Plugging into (2.2.35), we get an inequality

$$
\alpha_{\ell j} \leqslant a_{\ell j}+C \delta^{2} \sum_{m=0}^{\ell} \alpha_{m j} 2^{-m} .
$$

Using that $a_{\ell j}$ is increasing in $\ell$, we deduce from (2.2.36) that if $\delta$ is small enough $\alpha_{\ell j} \leqslant 2 a_{\ell j}$ which is the conclusion.

The last step of the proof is to estimate the right hand side of (2.2.33).

Proposition 2.2.9. - One has the following estimates, for a uniform constant $C$ :

$$
\begin{aligned}
\|\| \int \Gamma_{m}(t)\left|\widehat{f}_{j q}(t, \xi)\right| \frac{d t}{t} & \left\|_{L^{2}(d \xi)}\right\|_{\ell_{q}^{2}} \\
& \leqslant C \inf \left[\frac{2^{(m-j) / 2}}{\sqrt{\delta}}, 1\right] \sum_{p \in \mathbb{Z}^{d}}\left\|\Gamma_{m}(t) \phi_{p, \delta} \Delta_{j} f\right\|_{L^{2}\left(\frac{d t}{t} d x\right)}
\end{aligned}
$$

for any $m \in \mathbb{N}, j \geqslant-1$.

Proof. - We use the notation $\psi_{r, \delta}(t, \xi)$ introduced in the statement of proposition 2.2.6. We also denote by $\widetilde{\sim}_{r, \delta}(t, \xi)$ the function defined like $\psi_{r, \delta}(t, \xi)$, replacing $\psi$ by a function $\widetilde{\psi} \in C_{0}^{\infty}\left(\mathbb{R}^{d}\right), \widetilde{\psi} \geqslant 0, \widetilde{\psi} \equiv 1$ on $\operatorname{Supp} \psi$. Write

$$
\int \Gamma_{m}(t)\left|\widehat{f}_{j q}(t, \xi)\right| \frac{d t}{t} \leqslant C \sum_{r \in \mathbb{Z}^{d}} \sum_{j^{\prime}} \int \Gamma_{m}(t)\left|\psi_{r, \delta}(t, \xi) \varphi\left(2^{-j^{\prime}} \xi\right) \widehat{f}_{j q}(t, \xi)\right| \frac{d t}{t}
$$


where $\varphi\left(2^{-j^{\prime}} \xi\right)$ should be read $\chi(\xi)$ when $j^{\prime}=-1$. The general term of the above sum can be estimated by

$$
\begin{aligned}
\left(\int \widetilde{\varphi}\left(2^{-j^{\prime}} \xi\right) \widetilde{\psi}_{r, \delta}(t, \xi)^{2}\right. & \left.\Gamma_{m}(t) \frac{d t}{t}\right)^{1 / 2} \\
& \times\left(\int \Gamma_{m}(t) \psi_{r, \delta}(t, \xi)^{2} \varphi\left(2^{-j^{\prime}} \xi\right)^{2}\left|\widehat{f}_{j q}(t, \xi)\right|^{2} \frac{d t}{t}\right)^{1 / 2}
\end{aligned}
$$

By the same computation as in the proof of corollary 2.2.7, the first factor is smaller than $C \inf \left[1,2^{\left(m-j^{\prime}\right) / 2} / \sqrt{\delta}\right]$. The left hand side of $(2.2 .37)$ will be smaller than

$$
\begin{aligned}
& \sum_{j^{\prime}} C \inf \left[\frac{2^{\left(m-j^{\prime}\right) / 2}}{\sqrt{\delta}}, 1\right] \\
& \times\left\|\sum_{r}\right\| \Gamma_{m}(t) \psi_{r, \delta}(t, D) \Delta_{j^{\prime}} a_{q, \delta}(t, x, D) \Delta_{j} f\left\|_{L^{2}\left(\frac{d t}{t} d x\right)}\right\|_{\ell_{q}^{2}}
\end{aligned}
$$

Let us write

$$
\begin{aligned}
\Gamma_{m}(t) \psi_{r, \delta}(t, D) & \Delta_{j^{\prime}} a_{q, \delta}(t, x, D) \Delta_{j} f \\
& =\sum_{\ell, \ell^{\prime}, \ell^{\prime \prime}} \sum_{p} K_{\ell, \ell^{\prime}, \ell^{\prime \prime}, p, q}^{j, j^{\prime}, r}(t, x, D)\left(\Gamma_{m}(t) \phi_{\ell^{\prime \prime}, \delta}(D) \phi_{p, \delta}(x) \Delta_{j} f\right)
\end{aligned}
$$

where

$$
\begin{aligned}
& K_{\ell, \ell^{\prime}, \ell^{\prime \prime}, p, q}^{j, j^{\prime}, r}(t, x, D) \\
& \quad=\Delta_{j^{\prime}} \psi_{r, \delta}(t, D) \phi_{\ell, \delta}(D) a_{q, \delta}(t, x, D) \widetilde{\Delta}_{j} \phi_{\ell^{\prime}, \delta}(D) \widetilde{\phi}_{p, \delta}(x) \widetilde{\phi}_{\ell^{\prime \prime}, \delta}(D) .
\end{aligned}
$$

We write

$$
\begin{aligned}
& \left\|\Gamma_{m}(t) \psi_{r, \delta}(t, D) \Delta_{j^{\prime}} a_{q, \delta}(t, x, D) \Delta_{j} f\right\|_{L^{2}\left(\frac{d t}{t} d x\right)} \\
& \quad \leqslant C\|\| \sum_{\ell^{\prime}, \ell^{\prime \prime}, p} K_{\ell, \ell^{\prime}, \ell^{\prime \prime}, p, q}^{j, j^{\prime}, r}(t, x, D)\left(\Gamma_{m}(t) \phi_{\ell^{\prime \prime}, \delta}(D) \phi_{p, \delta}(x) \Delta_{j} f\right)\left\|_{L^{2}\left(\frac{d t}{t} d x\right)}\right\|_{\ell_{\ell}^{2}}
\end{aligned}
$$

since the $\ell$-sum is an almost orthogonal one. By theorem 2.1.3 and (2.2.42) the symbol $K_{\ell, \ell^{\prime}, \ell^{\prime \prime}, p, q}^{j,,^{\prime}, r}(t, x, \xi) \in S\left(A^{-\infty}\right)$ where

$$
A=\left\langle 2 \delta \xi\left(\frac{1}{t}-1\right)-r\right\rangle\langle\delta \xi-\ell\rangle\left\langle\delta\left(x+2 \xi\left(\frac{1}{t}-1\right)\right)-q\right\rangle\left\langle\delta \xi-\ell^{\prime}\right\rangle\langle\delta x-p\rangle\left\langle\delta \xi-\ell^{\prime \prime}\right\rangle
$$

with uniformly controlled semi-norms. This implies that the symbol is in $S(1)=S_{0,0}^{0}$ with semi-norms controlled by

$$
C_{N}\left\langle 2 \ell^{\prime \prime}\left(\frac{1}{t}-1\right)-r\right\rangle^{-N}\left\langle\ell-\ell^{\prime}\right\rangle^{-N}\left\langle\ell-\ell^{\prime \prime}\right\rangle^{-N}\langle r-(q-p)\rangle^{-N}
$$

uniformly in $\delta \in] 0,1], t \geqslant 1$. At fixed $t$, the $\mathcal{L}\left(L^{2}\right)$ operator norm of $K_{\ell, \ell^{\prime}, \ell^{\prime \prime}, p, q}^{j, j^{\prime}, r}(t, x, D)$ is thus smaller than (2.2.44). Moreover, since (2.2.42) implies that $\ell$ (resp. $\ell^{\prime}$ ) is of 
magnitude $2^{j^{\prime}}\left(\right.$ resp. $\left.2^{j}\right)$, the $\left\langle\ell-\ell^{\prime}\right\rangle^{-N}$ term of (2.2.44) provides a $2^{-N\left|j-j^{\prime}\right|}$ factor. At fixed $t$, we thus have

$$
\begin{aligned}
& \sum_{\ell^{\prime \prime}}\left\|\sum_{\ell^{\prime}, p} K_{\ell, \ell^{\prime}, \ell^{\prime \prime}, p, q}^{j, j^{\prime}, r}(t, x, D)\left(\Gamma_{m} \phi_{\ell^{\prime \prime}, \delta}(D) \phi_{p, \delta}(x) \Delta_{j} f\right)\right\|_{L^{2}(d x)} \\
& \leqslant C_{N} \sum_{\ell^{\prime}, \ell^{\prime \prime}, p}\left\langle 2 \ell^{\prime \prime}\left(\frac{1}{t}-1\right)-r\right\rangle^{-N}\left\langle\ell-\ell^{\prime}\right\rangle^{-N}\left\langle\ell-\ell^{\prime \prime}\right\rangle^{-N}\langle r-(q-p)\rangle^{-N} \\
& \times 2^{-N\left|j-j^{\prime}\right|}\left\|\Gamma_{m}(t) \phi_{\ell^{\prime \prime}, \delta}(D) \phi_{p, \delta}(x) \Delta_{j} f(t, \cdot)\right\|_{L^{2}(d x)} .
\end{aligned}
$$

Denote by

$$
\begin{aligned}
k_{\ell, \ell^{\prime \prime}, q}^{j, j^{\prime}, r}(t) & =\left\|\sum_{\ell^{\prime}, p} K_{\ell, \ell^{\prime}, \ell^{\prime \prime}, p, q}^{j, j^{\prime}, r}(t, x, D)\left(\Gamma_{m} \phi_{\ell^{\prime \prime}, \delta}(D) \phi_{p, \delta}(x) \Delta_{j} f\right)\right\|_{L^{2}(d x)} \\
\gamma_{\ell^{\prime \prime}, p}^{j}(t) & =\left\|\Gamma_{m}(t) \phi_{\ell^{\prime \prime}, \delta}(D) \phi_{p, \delta}(x) \Delta_{j} f(t, \cdot)\right\|_{L^{2}(d x)} .
\end{aligned}
$$

The norm to estimate in (2.2.40) is by (2.2.43) bounded from above by

$$
\left\|\sum_{r}\right\|\left\|\sum_{\ell^{\prime \prime}} k_{\ell, \ell^{\prime \prime}, q}^{j, j^{\prime}, r}(t)\right\|_{L^{2}(d t / t)}\left\|_{\ell_{\ell}^{2}}\right\|_{\ell_{q}^{2}} .
$$

Using (2.2.45), this last term can be controlled by

$$
\begin{aligned}
\left\|\sum_{r}\right\| \sum_{\ell^{\prime}, \ell^{\prime \prime}, p}\left\langle\ell-\ell^{\prime}\right\rangle^{-N}\left\langle\ell-\ell^{\prime \prime}\right\rangle^{-N}\langle r-(q-p)\rangle^{-N} 2^{-N\left|j-j^{\prime}\right|} \\
\times\left(\int\left\langle 2 \ell^{\prime \prime}\left(\frac{1}{t}-1\right)-r\right\rangle^{-2 N}\left|\gamma_{\ell^{\prime \prime}, p}^{j}(t)\right|^{2} \frac{d t}{t}\right)^{1 / 2}\left\|_{\ell_{\ell}^{2}}\right\|_{\ell_{q}^{2}} .
\end{aligned}
$$

Put

$$
\begin{aligned}
\beta_{\ell^{\prime \prime}, r, p}^{j} & =\left(\int\left\langle 2 \ell^{\prime \prime}\left(\frac{1}{t}-1\right)-r\right\rangle^{-2 N}\left|\gamma_{\ell^{\prime \prime}, p}^{j}(t)\right|^{2} \frac{d t}{t}\right)^{1 / 2} \\
\widetilde{\beta}_{r, p}^{j} & =\left\|\beta_{\ell^{\prime \prime}, r, p}^{j}\right\|_{\ell_{\ell^{\prime \prime}}^{2}} .
\end{aligned}
$$

We have an upper bound for (2.2.47)

$$
\begin{array}{r}
2^{-N\left|j-j^{\prime}\right|}\left\|\sum_{r}\right\| \sum_{\ell^{\prime \prime}, p}\left\langle\ell-\ell^{\prime \prime}\right\rangle^{-N}\langle r-(q-p)\rangle^{-N} \beta_{\ell^{\prime \prime}, r, p}^{j}\|\|_{\ell_{\ell}^{2}} \|_{\ell_{q}^{2}} \\
\leqslant C 2^{-N\left|j-j^{\prime}\right|}\left\|\sum_{r} \sum_{p}\langle r-(q-p)\rangle^{-N} \widetilde{\beta}_{r, p}^{j}\right\|_{\ell_{q}^{2}} \\
\leqslant C 2^{-N\left|j-j^{\prime}\right|} \sum_{p}\left\|\sum_{r}\langle r-q\rangle^{-N} \widetilde{\beta}_{r, p}^{j}\right\|_{\ell_{q}^{2}} \\
\leqslant C 2^{-N\left|j-j^{\prime}\right|} \sum_{p}\left\|\widetilde{\beta}_{r, p}^{j}\right\|_{\ell_{r}^{2}}
\end{array}
$$




$$
\begin{aligned}
\leqslant C 2^{-N\left|j-j^{\prime}\right|} \sum_{p}\left(\sum_{r}\right. & \left.\sum_{\ell^{\prime \prime}} \int\left\langle 2 \ell^{\prime \prime}\left(\frac{1}{t}-1\right)-r\right\rangle^{-2 N}\left|\gamma_{\ell^{\prime \prime}, p}^{j}(t)\right|^{2} \frac{d t}{t}\right)^{1 / 2} \\
& \leqslant C 2^{-N\left|j-j^{\prime}\right|} \sum_{p}\left\|\Gamma_{m} \phi_{p, \delta}(x) \Delta_{j} f\right\|_{L^{2}\left(\frac{d t}{t} d x\right)} .
\end{aligned}
$$

Going back to (2.2.40) we see that we proved that the left hand side of (2.2.37) is smaller than

$$
C \sum_{j^{\prime}} \inf \left[2^{\left(m-j^{\prime}\right) / 2} / \sqrt{\delta}, 1\right] 2^{-N\left|j-j^{\prime}\right|} \sum_{p}\left\|\Gamma_{m}(t) \phi_{p, \delta}(x) \Delta_{j} f\right\|_{L^{2}\left(\frac{d t}{t} d x\right)} .
$$

The conclusion of the proposition follows.

Proof of theorem 2.2.4. - One has just to apply corollary 2.2 .7 followed by proposition 2.2.8 and proposition 2.2.9, remarking that we have a bound for \|\|$\widehat{v}_{j q}^{0}(\xi)\left\|_{L^{2}(d \xi)}\right\|_{\ell_{q}^{2}}$ in terms of $\left\|\Delta_{j} v_{0}\right\|_{L^{2}}$.

Proof of theorem 2.2.3. - From now on, we shall fix $\delta$ small enough so that inequality (2.2.16) of theorem 2.2.4 holds true. We can then, in the left and right hand sides of (2.2.16), replace $\phi_{p, \delta}$ by $\phi_{p}$, loosing a bigger constant in the right hand side. Let us prove first that $\|v\|_{F_{\alpha, \alpha^{\prime}, \infty}^{s, s^{\prime}+1 / 2}}$ is smaller than the right hand side of (2.2.15). We deduce from (2.2.16) that for any $q$

$$
\begin{array}{r}
2^{-\left(j-\frac{\ell}{2}\right) \alpha}\left(1+2^{j-\frac{\ell}{2}}\right)^{s+\alpha} 2^{-(j-\ell) \alpha^{\prime}}\left(1+2^{j-\ell}\right)^{s^{\prime}+\frac{1}{2}+\alpha^{\prime}}\left\|\Gamma_{\ell}(t) \phi_{q} \Delta_{j} v\right\|_{L^{2}\left(\frac{d t}{t} d x\right)} \\
\leqslant C a_{j \ell}\left\|\Delta_{j} v_{0}\right\|_{L^{2}} 2^{j\left(s+s^{\prime}\right)} \\
+C \sum_{m=0}^{\ell} b_{j \ell m} 2^{-(j-m / 2) \alpha}\left(1+2^{j-m / 2}\right)^{s+\alpha} 2^{-(j-m) \alpha^{\prime}}\left(1+2^{j-m}\right)^{s^{\prime}-1 / 2+\alpha^{\prime}} \\
\times\|\| \Gamma_{m} \phi_{p}(x) \Delta_{j} f\left\|_{L^{2}\left(\frac{d t}{t} d x\right)}\right\|_{\ell_{p}^{1}}
\end{array}
$$

with

$$
\begin{gathered}
a_{j \ell}=2^{-j\left(s+s^{\prime}+\alpha+\alpha^{\prime}\right)+\ell\left(\alpha+2 \alpha^{\prime}\right) / 2}\left(1+2^{j-\ell / 2}\right)^{s+\alpha}\left(1+2^{j-\ell}\right)^{s^{\prime}+\alpha^{\prime}} \\
b_{j \ell m}=2^{(\ell-m)\left(\alpha+2 \alpha^{\prime}\right) / 2}\left(1+2^{j-\ell / 2}\right)^{s+\alpha}\left(1+2^{j-m / 2}\right)^{-s-\alpha} \\
\times\left(1+2^{j-\ell}\right)^{s^{\prime}+\alpha^{\prime}}\left(1+2^{j-m}\right)^{-s^{\prime}-\alpha^{\prime}} .
\end{gathered}
$$

Since $s+\alpha \geqslant 0, s^{\prime}+\alpha^{\prime} \geqslant 0$, we get $a_{j \ell} \leqslant C 2^{\ell\left(\alpha+2 \alpha^{\prime}\right) / 2}$ and $b_{j \ell m} \leqslant 2^{(\ell-m)\left(\alpha+2 \alpha^{\prime}\right) / 2}$. Since $\frac{\alpha}{2}+\alpha^{\prime}<0$, we get taking the $\ell_{j}^{2} \ell_{q}^{\infty}$ norm of $(2.2 .49)$

$$
\begin{aligned}
\|v\|_{F_{\alpha, \alpha^{\prime}, \infty}^{s, s^{\prime}+1 / 2}} & \leqslant C\left\|v_{0}\right\|_{H^{s+s^{\prime}}}+C \sum_{m=0}^{\ell} 2^{(\ell-m)\left(\alpha+2 \alpha^{\prime}\right) / 2}\|f\|_{F_{\alpha, \alpha^{\prime}, 1}^{s, s^{\prime}-1 / 2}} \\
& \leqslant C\left(\left\|v_{0}\right\|_{H^{s+s^{\prime}}}+\|f\|_{F_{\alpha, \alpha^{\prime}, 1}^{s, 1 / 2}} .\right.
\end{aligned}
$$


We still have to prove that $v \in \underline{H}_{\alpha, \alpha^{\prime}}^{s, s^{\prime}}$. Using inequality (2.1.15) with $r=2$ we get for any fixed $t$

$$
\begin{aligned}
\left\|\Delta_{j} v(t, \cdot)\right\|_{L^{2}(d x)} & \leqslant C\|\| \phi_{p} \Delta_{j} v(t, \cdot)\left\|_{L^{2}(d x)}\right\|_{\ell_{p}^{2}} \\
& \leqslant C\|\|\left\|\phi_{p} a_{q}(t, x, D) \Delta_{j} v(t, \cdot)\right\|_{L^{2}(d x)}\left\|_{\ell_{q}^{2}}\right\|_{\ell_{p}^{2}} \\
& \leqslant C\|\| \mathcal{F}\left(a_{q}(t, x, D) \Delta_{j} v\right)(t, \xi)\left\|_{L^{2}(d \xi)}\right\|_{\ell_{q}^{2}}
\end{aligned}
$$

Using notations (2.2.32), for $t$ in the support of $\Gamma_{\ell}(t)$, we can bound $\left\|\Delta_{j} v(t, \cdot)\right\|_{L^{2}}$ by (2.2.33), whence using estimate (2.2.37) the inequality

$$
\left\|\Gamma_{\ell}(t) \Delta_{j} v(t, \cdot)\right\|_{L^{2}} \leqslant C\left[\left\|\Delta_{j} v_{0}\right\|_{L^{2}}+\sum_{m=0}^{\ell}\left(1+2^{j-m}\right)^{-1 / 2}\|\| \Gamma_{m}(t) \phi_{p} \Delta_{j} f\left\|_{L^{2}\left(\frac{d t}{t} d x\right)}\right\|_{\ell_{p}^{1}}\right] .
$$

Arguing as for inequality (2.2.49), we deduce from that

$$
\|v\|_{\underline{H}_{\alpha, \alpha^{\prime}}^{s, s^{\prime}}} \leqslant C\left[\left\|v_{0}\right\|_{H^{s+s^{\prime}}}+\|f\|_{F_{\alpha, \alpha^{\prime}, 1}^{s, s^{\prime}-1 / 2}}\right] \text {. }
$$

This concludes the proof of theorem 2.2.3. 



\section{CHAPTER 3}

\section{NONLINEAR ESTIMATES}

\subsection{Estimates for products}

The aim of this section is to study regularity of products in the framework of spaces $F_{\alpha, \alpha^{\prime}, r}^{s, s^{\prime}}$ and $H_{\alpha, \alpha^{\prime}, r}^{s, s^{\prime}}$ introduced in definition 2.2.1. Let us define a few more notations: let $\chi \in C_{0}^{\infty}\left(\mathbb{R}^{d}\right), \chi \equiv 1$ close to 0 .

Definition 3.1.1. - One denotes by $\widetilde{H}_{\alpha, \alpha^{\prime}, r}^{s, s^{\prime}}\left(\operatorname{resp} . \underline{\widetilde{H}}_{\alpha, \alpha^{\prime}}^{s, s^{\prime}}\right)$ the space of those $v \in$ $H_{\alpha, \alpha^{\prime}, r}^{s, s^{\prime}}$ (resp. in $\left.\underline{H}_{\alpha, \alpha^{\prime}}^{s, s^{\prime}}\right)$ such that

$$
\left(\left\|\phi_{q}(x) \chi(D / \sqrt{t}) v(t, x)\right\|_{L^{\infty}(d t d x)}\right)_{q} \in \ell_{q}^{r},
$$

(resp.

$$
\left.\chi(D / \sqrt{t}) v(t, x) \in L^{\infty}(d t d x)\right) .
$$

The above definition depends on the choice of $\chi$ except in the case $\alpha^{\prime}>d / 2$, which is the only one we will encounter:

Lemma 3.1.2. - Assume $\alpha^{\prime}>d / 2$. Then definition 3.1.1 is independent of the choice of $\chi$. More generally, if $\psi(t, \xi)$ is a smooth function satisfying $\operatorname{Supp} \psi \subset$ $\{(t, \xi) ;|\xi|<C \sqrt{t}\}$ and $\left|\partial_{\xi}^{\beta} \psi(t, \xi)\right| \leqslant C_{\beta} t^{-|\beta| / 2}$ for any $\beta \in \mathbb{N}^{d}$, we have for $v \in \widetilde{H}_{\alpha, \alpha^{\prime}, r}^{s, s^{\prime}}$ (resp. $\left.v \in \underline{\widetilde{H}}_{\alpha, \alpha^{\prime}}^{s, s^{\prime}}\right)$

$$
\left(\left\|\phi_{q}(x) \psi(t, D) v\right\|_{L^{\infty}(d t d x)}\right)_{q} \in \ell_{q}^{r}
$$

(resp.

$$
\left.\psi(t, D) v \in L^{\infty}(d t d x) .\right)
$$

If, moreover, $\alpha+2 \alpha^{\prime}>0$, then $\widetilde{H}_{\alpha, \alpha^{\prime}, r}^{s, s^{\prime}}=H_{\alpha, \alpha^{\prime}, r}^{s, s^{\prime}}$ (resp. $\underline{\widetilde{H}}_{\alpha, \alpha^{\prime}}^{s, s^{\prime}}=\underline{H}_{\alpha, \alpha^{\prime}}^{s, s^{\prime}}$ ) and one has more precisely

$$
\left(\left\|\phi_{q}(x) t^{\delta} \chi(D / \sqrt{t}) v\right\|_{L^{\infty}(d t d x)}\right)_{q} \in \ell_{q}^{r}
$$


(resp.

$$
\left.t^{\delta} \chi(D / \sqrt{t}) v \in L^{\infty}(d t d x)\right)
$$

for any $\delta \in\left[0, \min \left(\frac{\alpha}{2}+\alpha^{\prime}, \frac{\alpha^{\prime}}{2}-\frac{d}{4}\right)[\right.$.

Proof. - For $v$ satisfying (3.1.1), write

$$
\phi_{q} \psi(t, D) v=\phi_{q} \psi(t, D) \chi(D / \sqrt{t}) v+\phi_{q} \psi(t, D)(1-\chi)(D / \sqrt{t}) v .
$$

We shall again denote by $\widetilde{\phi}_{q^{\prime}}$ the function $\widetilde{\phi}_{q^{\prime}}(x)=\widetilde{\phi}\left(x-q^{\prime}\right)$ for $\widetilde{\phi} \in C_{0}^{\infty}\left(\mathbb{R}^{d}\right), \widetilde{\phi} \equiv 1$ close to $\operatorname{Supp} \phi$. Let us remark that an operator of form $K_{q, q^{\prime}}^{t}=\phi_{q}(x) \circ \psi(t, D) \circ \widetilde{\phi}_{q^{\prime}}(x)$ has a kernel whose modulus is bounded from above by

$$
C t^{d / 2}(1+\sqrt{t}|x-y|)^{-2 N}\left|\phi_{q}(x)\right|\left|\widetilde{\phi}_{q^{\prime}}(y)\right| \leqslant C t^{d / 2}(1+\sqrt{t}|x-y|)^{-N}\left\langle q-q^{\prime}\right\rangle^{-N}
$$

whence

$$
\begin{gathered}
\left\|K_{q q^{\prime}}^{t}\right\|_{\mathcal{L}\left(L^{\infty}\right)} \leqslant C_{N}\left\langle q-q^{\prime}\right\rangle^{-N} \\
\left\|K_{q q^{\prime}}^{t}\right\|_{\mathcal{L}\left(L^{2}, L^{\infty}\right)} \leqslant C_{N} t^{d / 4}\left\langle q-q^{\prime}\right\rangle^{-N} .
\end{gathered}
$$

We estimate the first contribution to (3.1.7) by

$$
\begin{aligned}
\left\|\phi_{q} \psi(t, D) \chi(D / \sqrt{t}) v\right\|_{L^{\infty}} & \leqslant \sum_{q^{\prime}}\left\|\left(\phi_{q} \psi(t, D) \widetilde{\phi}_{q^{\prime}}\right)\left(\phi_{q^{\prime}} \chi(D / \sqrt{t}) v\right)\right\|_{L^{\infty}(d t d x)} \\
& \leqslant \sum_{q^{\prime}} C_{N}\left\langle q-q^{\prime}\right\rangle^{-N}\left\|\phi_{q^{\prime}} \chi(D / \sqrt{t}) v\right\|_{L^{\infty}(d t d x)}
\end{aligned}
$$

which shows that this sequence is in $\ell_{q}^{r}$ because of assumption (3.1.1). The second contribution to the right hand side of (3.1.7) is estimated at fixed $t$ by

$$
\begin{aligned}
& \left\|\phi_{q} \psi(t, D)(1-\chi)(D / \sqrt{t}) v\right\|_{L^{\infty}} \\
& \quad \leqslant \sum_{q^{\prime}} \sum_{j}\left\|\left(\phi_{q} \psi(t, D)(1-\chi)(D / \sqrt{t}) \widetilde{\phi}_{q^{\prime}}\right)\left(\phi_{q^{\prime}} \Delta_{j} v\right)(t, \cdot)\right\|_{L^{\infty}(d x)}
\end{aligned}
$$

where the $j$-sum is restricted to indices satisfying $C_{1} \sqrt{t}<2^{j}<C_{2} \sqrt{t}$. By (3.1.9), we get the upper bound

$$
\begin{aligned}
\sum_{q^{\prime}} \sum_{j ; 2^{j} \sim \sqrt{t}} 2^{j d / 2}\left\langle q-q^{\prime}\right\rangle^{-N}\left\|\phi_{q^{\prime}} \Delta_{j} v(t, \cdot)\right\|_{L^{2}(d x)} & \\
& \leqslant \sum_{q^{\prime}} \sum_{j ; 2^{j} \sim \sqrt{t}} 2^{j d / 2}\left\langle q-q^{\prime}\right\rangle^{-N} c_{j q^{\prime}}\left(2^{j} / \sqrt{t}\right)^{\alpha}\left(2^{j} / t\right)^{\alpha^{\prime}}
\end{aligned}
$$

for a sequence $\left(c_{j q^{\prime}}\right)_{j q^{\prime}}$ in $\ell_{j}^{2} \ell_{q^{\prime}}^{r}$ using the definition of $H_{\alpha, \alpha^{\prime}, r}^{s, s^{\prime}}$. The supremum in $t$ of this last quantity is smaller than $C c_{q}^{\prime}$ with $\left(c_{q}^{\prime}\right)_{q} \in \ell_{q}^{r}$ since $\alpha^{\prime}>d / 2$.

Finally, when $\alpha+2 \alpha^{\prime}>0,\left\|\phi_{q} \chi(D / \sqrt{t}) v\right\|_{L^{\infty}(d x)}$ is bounded from above by the sum in the right hand side of (3.1.11), extended to $2^{j}<C \sqrt{t}$ i.e by

$$
\sum_{2^{j}<C \sqrt{t}} 2^{j\left(\frac{d}{2}+\alpha+\alpha^{\prime}\right)} t^{-\alpha / 2-\alpha^{\prime}} c_{j q} \leqslant \frac{C}{t^{\delta}} c_{q}^{\prime}
$$


for $0 \leqslant \delta<\min \left[\frac{\alpha}{2}+\alpha^{\prime}, \frac{\alpha^{\prime}}{2}-\frac{d}{4}\right]$ and $\left(c_{q}^{\prime}\right)_{q} \in \ell^{r}$.

The proof for $v \in \underline{\tilde{H}}_{\alpha, \alpha^{\prime}}^{s, s^{\prime}}$ is similar and simpler.

We will study products of elements of $F_{\alpha, \alpha^{\prime}, r}^{s, s^{\prime}}, H_{\alpha, \alpha^{\prime}, r}^{s, s^{\prime}}$ using Bony's paraproducts [1]. Define for $u, v \in \mathcal{S}^{\prime}\left(\mathbb{R}^{d}\right)$

$$
T_{u} v=\sum_{j \geqslant 1} S_{j-1} u \Delta_{j} v
$$

The series converge in $\mathcal{S}^{\prime}\left(\mathbb{R}^{d}\right)$ and each of its terms has Fourier transform supported inside a ring of size $C 2^{j}$. Moreover, we set

$$
R(u, v)=\sum_{j, j^{\prime} ;\left|j-j^{\prime}\right| \leqslant 1} \Delta_{j} u \Delta_{j^{\prime}} v
$$

when the above series converges. We will use the same notations when $u, v$ depend on the parameter $t \in[1,+\infty[$. When (3.1.13) has a meaning, one gets Bony's decomposition of the product $u v$ :

$$
u v=T_{u} v+T_{v} u+R(u, v) .
$$

We will also use notations similar to (3.1.12), (3.1.13) in a slightly more general framework. Assume we are given constants $C_{1}>0, C_{2}>0$ and families of $C^{\infty}$ functions $\left(\varphi_{j}(\xi)\right)_{j \in \mathbb{N}},\left(\chi_{j}(\xi)\right)_{j \in \mathbb{N}}$ satisfying

$$
\begin{aligned}
& \operatorname{Supp} \varphi_{j}(\xi) \subset\left\{\xi ; C_{1}^{-1} 2^{j}<|\xi|<C_{1} 2^{j}\right\}, \quad\left|\partial_{\xi}^{\beta} \varphi_{j}(\xi)\right| \leqslant C_{\beta} 2^{-j|\beta|} \\
& \operatorname{Supp} \chi_{j}(\xi) \subset\left\{\xi ;|\xi|<C_{2} 2^{j}\right\}, \quad\left|\partial_{\xi}^{\beta} \chi_{j}(\xi)\right| \leqslant C_{\beta} 2^{-j|\beta|} .
\end{aligned}
$$

We will use the generic notation $\widetilde{T}_{u} v$ and $\widetilde{R}(u, v)$ for operators

$$
\begin{aligned}
\widetilde{T}_{u} v & =\sum_{j \geqslant N_{1}}\left(\chi_{j-N_{1}}(D) u\right)\left(\varphi_{j}(D) v\right) \\
\widetilde{R}(u, v) & =\sum_{j, j^{\prime} ;\left|j-j^{\prime}\right| \leqslant N_{2}}\left(\varphi_{j}(D) u\right)\left(\varphi_{j^{\prime}}(D) v\right)
\end{aligned}
$$

with $N_{1}, N_{2} \in \mathbb{N}$, with $N_{1}$ large enough so that the Fourier transform of each contribution to $\widetilde{T}_{u} v$ be supported inside a ring of size $2^{j}$. In the definition of $\widetilde{R}$, we also eventually allow low frequency contributions of type $\left(\chi_{0}(D) u\right)\left(\varphi_{j^{\prime}}(D) v\right),\left|j^{\prime}\right| \leqslant N_{2}$, $\left(\varphi_{j}(D) u\right)\left(\chi_{0}(D) v\right),|j| \leqslant N_{2}$.

\section{Proposition 3.1.3}

(i) Let $u \in L^{\infty}\left(\frac{d t}{t} d x\right)$ and $v \in F_{\alpha, \alpha^{\prime}, r}^{s, s^{\prime}}$ (resp. $v \in H_{\alpha, \alpha^{\prime}, r}^{s, s^{\prime}}$. Then $T_{u} v$ and $\widetilde{T}_{u} v$ are in $F_{\alpha, \alpha^{\prime}, r}^{s, s^{\prime}}\left(\right.$ resp. $\left.H_{\alpha, \alpha^{\prime}, r}^{s, s^{\prime}}\right)$.

(ii) Let $s_{j}, s_{j}^{\prime}, \alpha_{j}, \alpha_{j}^{\prime}, r_{j}, j=1,2$ be indices with $r_{j} \in[1,+\infty]$. Assume $\alpha_{1}^{\prime}>$ $d / 2, s_{1} \neq d$. Let $u \in \widetilde{H}_{\alpha_{1}, \alpha_{1}^{\prime}, r_{1}}^{s_{1}, s_{1}^{\prime}}, v \in F_{\alpha_{2}, \alpha_{2}^{\prime}, r_{2}}^{s_{2}, s_{2}^{\prime}}$ (resp. $v \in \underline{H}_{\alpha_{2}, \alpha_{2}^{\prime}}^{s_{2}, s_{2}^{\prime}}$. Then $T_{u} v$ and 
$\widetilde{T}_{u} v$ belong to $F_{\alpha, \alpha^{\prime}, r}^{s, s^{\prime}}\left(\right.$ resp. $\left.H_{\alpha, \alpha^{\prime}, r_{1}}^{s, s^{\prime}}\right)$ where

$$
\begin{aligned}
\alpha & =\alpha_{2}+\left(\alpha_{1}+2 \alpha_{1}^{\prime}\right)_{+}, \quad \alpha^{\prime}=\alpha_{2}^{\prime}, \\
s & =s_{2}-\left(d-s_{1}\right)_{+}, \quad s^{\prime}=s_{2}^{\prime}-\left[-s_{1}^{\prime}-\frac{d}{2}\right]_{+}, \\
\frac{1}{r} & =\min \left[1, \frac{1}{r_{1}}+\frac{1}{r_{2}}\right] .
\end{aligned}
$$

Proof

(i) For fixed $t$ we write

$$
\left\|\phi_{q}(x) \Delta_{j}\left(T_{u} v\right)(t, \cdot)\right\|_{L^{2}(d x)} \leqslant \sum_{j^{\prime} ;\left|j-j^{\prime}\right| \leqslant N_{0}}\left\|\phi_{q}(x) \Delta_{j}\left(S_{j^{\prime}-1} u \Delta_{j^{\prime}} v\right)(t, \cdot)\right\|_{L^{2}(d x)}
$$

for a large enough integer $N_{0}$. The general term of the above sum is smaller than

$$
\sum_{q^{\prime}}\left\|\left(\phi_{q} \Delta_{j} \widetilde{\phi}_{q^{\prime}}\right)\left(S_{j^{\prime}-1} u \phi_{q^{\prime}}(x) \Delta_{j^{\prime}} v\right)(t, \cdot)\right\|_{L^{2}(d x)} \cdot
$$

Using that the $\mathcal{L}\left(L^{2}\right)$ operator norm of the composition $\phi_{q} \Delta_{j} \widetilde{\phi}_{q^{\prime}}$ is smaller than $C_{N}\left\langle q-q^{\prime}\right\rangle^{-N}$ we get for (3.1.18) the upper bound

$$
\sum_{q^{\prime}} \sum_{j^{\prime} ;\left|j-j^{\prime}\right| \leqslant N_{0}} C_{N}\left\langle q-q^{\prime}\right\rangle^{-N}\left\|S_{j^{\prime}-1} u\right\|_{L^{\infty}}\left\|\phi_{q^{\prime}}(x) \Delta_{j^{\prime}} v(t, \cdot)\right\|_{L^{2}(d x)} .
$$

Since $\left\|S_{j^{\prime}-1} u\right\|_{L^{\infty}} \leqslant C\|u\|_{L^{\infty}}$ the result follows multiplying by a cut-off $\Gamma_{\ell}(t)$ and computing the $L^{2}(d t / t)$ (resp. $\left.L^{\infty}(d t)\right)$ norm.

(ii) To argue in the same way, we need an estimate for quantities like

$$
\left\|\phi_{q} S_{j} u(t, \cdot)\right\|_{L^{\infty}(d x)} .
$$

LEMMA 3.1.4. - Let $u \in \widetilde{H}_{\alpha, \alpha^{\prime}, r}^{s, s^{\prime}}$ with $\alpha^{\prime}>d / 2, s \neq d$. We have for any $j$ and any $t$ the estimate

$$
\begin{aligned}
\left\|\phi_{q}(x) S_{j} u(t, \cdot)\right\|_{L^{\infty}(d x)} \leqslant c_{j q}\left(2^{j} / \sqrt{t}\right)^{\left(\alpha+2 \alpha^{\prime}\right)} & \left(1+2^{j} / \sqrt{t}\right)^{-\left(\alpha+2 \alpha^{\prime}\right)_{+}+(d-s)_{+}} \\
& \times\left(1+2^{j} / t\right)^{\left[-\frac{d}{2}-s^{\prime}\right]+}\|u\|_{\widetilde{H}_{\alpha, \alpha^{\prime}, r}^{s, s^{\prime}}}
\end{aligned}
$$

where $\left(c_{j q}\right)_{j q}$ is a sequence in $\ell_{j}^{\infty} \ell_{q}^{r}$.

Proof. - Consider first $j, t$ with $2^{j}<\sqrt{t}$. When $\alpha+2 \alpha^{\prime} \leqslant 0$, we argue as in (3.1.10) to obtain

$$
\begin{aligned}
\left\|\phi_{q} S_{j} u(t, \cdot)\right\|_{L^{\infty}(d x)} & =\left\|\phi_{q} S_{j} \chi(D / \sqrt{t}) u(t, \cdot)\right\|_{L^{\infty}(d x)} \\
& \leqslant \sum_{q^{\prime}}\left\|\left(\phi_{q} S_{j} \widetilde{\phi}_{q^{\prime}}\right)\left(\phi_{q^{\prime}} \chi(D / \sqrt{t}) u(t, \cdot)\right)\right\|_{L^{\infty}(d x)} \\
& \leqslant C_{N} \sum_{q^{\prime}}\left\langle q-q^{\prime}\right\rangle^{-N}\left\|\phi_{q^{\prime}} \chi(D / \sqrt{t}) u(t, \cdot)\right\|_{L^{\infty}(d x)}
\end{aligned}
$$

MÉMOIRES DE LA SMF 91 
and we use condition (3.1.1) of the definition of $\widetilde{H}_{\alpha, \alpha^{\prime}, r}^{s, s^{\prime}}$ to get an upper bound in terms of a $\ell_{q}^{r}$ sequence. When $\alpha+2 \alpha^{\prime}>0$, we write instead

$$
\begin{aligned}
\left\|\phi_{q} S_{j} u(t, \cdot)\right\|_{L^{\infty}(d x)} & \leqslant \sum_{k \leqslant j-1}\left\|\phi_{q} \Delta_{k} u(t, \cdot)\right\|_{L^{\infty}(d x)} \\
& \leqslant \sum_{k \leqslant j-1} \sum_{q^{\prime}}\left\|\left(\phi_{q} \widetilde{\Delta}_{k} \widetilde{\phi}_{q^{\prime}}\right)\left(\phi_{q^{\prime}} \Delta_{k} u\right)\right\|_{L^{\infty}(d x)}
\end{aligned}
$$

where $\widetilde{\Delta}_{k}$ is such that $\widetilde{\Delta}_{k} \Delta_{k}=\Delta_{k}$. Since the $\mathcal{L}\left(L^{2}, L^{\infty}\right)$ operator norm of $\phi_{q} \widetilde{\Delta}_{k} \widetilde{\phi}_{q^{\prime}}$ is smaller than $C 2^{k d / 2}\left\langle q-q^{\prime}\right\rangle^{-N}$ we get the upper bound

$$
\begin{aligned}
C \sum_{k \leqslant j-1} \sum_{q^{\prime}} 2^{k d / 2}\left\langle q-q^{\prime}\right\rangle^{-N}\left\|\phi_{q^{\prime}} \Delta_{k} u(t, \cdot)\right\|_{L^{2}} \\
\quad \leqslant C \sum_{k \leqslant j-1} \sum_{q^{\prime}}\left\langle q-q^{\prime}\right\rangle^{-N} 2^{k d / 2}\left(2^{k} / \sqrt{t}\right)^{\alpha}\left(2^{k} / t\right)^{\alpha^{\prime}} c_{k q^{\prime}}
\end{aligned}
$$

with $\left(c_{k q^{\prime}}\right)_{k q^{\prime}} \in \ell_{k}^{2} \ell_{q^{\prime}}^{r}$. We write (3.1.21) as $\left(2^{j} / \sqrt{t}\right)^{\alpha+2 \alpha^{\prime}} d_{j q}$ with

$$
d_{j q}=\sum_{k} \mathbb{1}_{\{k \leqslant j-1\}} 2^{k \frac{d}{2}+k\left(\alpha+\alpha^{\prime}\right)-j\left(\alpha+2 \alpha^{\prime}\right)} c_{k q}^{\prime}
$$

with $\left(c_{k q}^{\prime}\right)_{k q}$ in $\ell_{k}^{2} \ell_{q}^{r}$. Since $\alpha+2 \alpha^{\prime}>0, \alpha^{\prime}>d / 2,\left(d_{j q}\right)_{j q}$ is a $\ell_{j}^{\infty} \ell_{q}^{r}$ sequence.

Consider next the case $C \sqrt{t}<2^{j}<C t$. Arguing as in (3.1.20), (3.1.21) we get the upper bound

$$
\begin{aligned}
\left\|\phi_{q} S_{j} u(t, \cdot)\right\|_{L^{\infty}} \leqslant\left\|\phi_{q} \chi(D / \sqrt{t}) u(t, \cdot)\right\|_{L^{\infty}(d x)} & \\
& +C_{N} \sum_{C \sqrt{t}<2^{k} \leqslant 2^{j-1}} \sum_{q^{\prime}} 2^{k d / 2}\left\langle q-q^{\prime}\right\rangle^{-N}\left\|\phi_{q^{\prime}} \Delta_{k} u(t, \cdot)\right\|_{L^{2}} .
\end{aligned}
$$

The first term in the right hand side is smaller than a $\ell_{q}^{r}$ sequence, uniformly in $t$, by definition of $\widetilde{H}_{\alpha, \alpha^{\prime}, r}^{s, s^{\prime}}$, and the second one is smaller than

$$
\sum_{q^{\prime}} \sum_{k} \mathbb{1}_{\left\{C \sqrt{t}<2^{k}<2^{j}<C t\right\}}\left\langle q-q^{\prime}\right\rangle^{-N} 2^{k d / 2}\left(2^{k} / \sqrt{t}\right)^{-s}\left(2^{k} / t\right)^{\alpha^{\prime}} c_{k q^{\prime}} .
$$

When $\frac{s}{2}-\alpha^{\prime} \geqslant 0$, the supremum in $t$ of (3.1.22) is smaller than the $\operatorname{sum} \sum_{k} 2^{k\left(\frac{d}{2}-\alpha^{\prime}\right)} c_{k q}^{\prime}$ for a $\ell_{k}^{2} \ell_{q}^{r}$ sequence $\left(c_{k q}^{\prime}\right)_{k q}$, and so smaller than a $\ell_{q}^{r}$ sequence since $\alpha^{\prime}>d / 2$.

When $\frac{s}{2}-\alpha^{\prime}<0$ and $d<s$, the supremum in $t$ of (3.1.22) is smaller than

$$
\sum_{k} \mathbb{1}_{\{k \leqslant j-1\}} 2^{k \frac{d}{2}-\left(k-\frac{j}{2}\right) s+(k-j) \alpha^{\prime}} c_{k q}^{\prime} \leqslant \sum_{k} 2^{\frac{k}{2}(d-s)} c_{k q}^{\prime}
$$

which is a $\ell_{q}^{r}$ sequence. Assume now $\frac{s}{2}-\alpha^{\prime}<0$ and $d>s$. We write (3.1.22) as $\left(2^{j} / \sqrt{t}\right)^{d-s} d_{j q}$ with

$$
d_{j q}=\sum_{k} \mathbb{1}_{\left\{C \sqrt{t}<2^{k}<2^{j}<C t\right\}} 2^{(k-j)(d-s)}\left(2^{k} / t\right)^{\alpha^{\prime}-\frac{d}{2}} c_{k q}^{\prime} .
$$


The supremum in $t$ is smaller than $C \sum_{k} \mathbb{1}_{\{k \leqslant j-1\}} 2^{(k-j)\left(\frac{d}{2}-s+\alpha^{\prime}\right)} c_{k q}^{\prime}$ which is a $\ell_{j}^{2} \ell_{q}^{r}$ sequence. This gives again (3.1.19).

Finally, consider the case $C t \leqslant 2^{j}$. We estimate

$$
\begin{aligned}
\left\|\phi_{q} S_{j} u(t, \cdot)\right\|_{L^{\infty}} \leqslant \| \phi_{q} \chi(D / t) & u(t, \cdot) \|_{L^{\infty}(d x)} \\
& +C_{N} \sum_{C t \leqslant 2^{k}<2^{j}} \sum_{q^{\prime}} 2^{k d / 2}\left\langle q-q^{\prime}\right\rangle^{-N}\left\|\phi_{q^{\prime}} \Delta_{k} u(t, \cdot)\right\|_{L^{2}} .
\end{aligned}
$$

The first term is estimated by the right hand side of (3.1.19), as easily seen reproducing the computations of the two preceding cases. We are left with estimating

$$
\sum_{C t<2^{k}<2^{j}} 2^{k d / 2}\left(2^{k} / \sqrt{t}\right)^{-s}\left(2^{k} / t\right)^{-s^{\prime}} c_{k q}^{\prime} \leqslant\left(2^{j} / t\right)^{\left[-\frac{d}{2}-s^{\prime}\right]_{+}} \sum_{C t<2^{k}<2^{j}}\left(2^{k} / \sqrt{t}\right)^{d-s} c_{k q}^{\prime}
$$

for a $\ell_{k}^{2} \ell_{q^{\prime}}^{r}$ sequence $\left(c_{k q}^{\prime}\right)_{k q}$. If $d-s>0$, the last sum is smaller than $\left(2^{j} / \sqrt{t}\right)^{d-s} c_{j q}^{\prime \prime}$ for a $\ell_{j}^{2} \ell_{q}^{r}$ sequence $\left(c_{j q}^{\prime \prime}\right)_{j q}$. If $d-s<0$, the supremum in $t$ of that sum is smaller than $\sum_{k} 2^{k(d-s) / 2} c_{k q}^{\prime}$ which is a $\ell_{q}^{r}$ sequence. In both cases, this gives the upper bound of (3.1.19).

End of the proof of proposition 3.1.3. - When $v \in F_{\alpha_{2}, \alpha_{2}^{\prime}, r_{2}}^{s_{2}, s_{2}^{\prime}}$, we estimate the general term of the right hand side of (3.1.18) by

$$
\begin{aligned}
& \sum_{q^{\prime}}\left\|\left(\phi_{q} \Delta_{j} \widetilde{\phi}_{q^{\prime}}\right)\left(\widetilde{\phi}_{q^{\prime}} S_{j^{\prime}-1} u\right)\left(\phi_{q^{\prime}} \Delta_{j^{\prime}} v\right)(t, \cdot)\right\|_{L^{2}(d x)} \\
& \quad \leqslant C_{N} \sum_{q^{\prime}}\left\langle q-q^{\prime}\right\rangle^{-N}\left\|\widetilde{\phi}_{q^{\prime}} S_{j^{\prime}-1} u(t, \cdot)\right\|_{L^{\infty}}\left\|\phi_{q^{\prime}} \Delta_{j^{\prime}} v(t, \cdot)\right\|_{L^{2}(d x)} .
\end{aligned}
$$

We just have to apply lemma 3.1.4 to get the conclusion.

When $v \in \underline{H}_{\alpha_{2}, \alpha_{2}^{\prime}}^{s_{2}, s^{\prime}}$, we bound the general term of the right hand side of (3.1.18) by

$$
\begin{aligned}
& \sum_{q^{\prime}}\left\|\left(\phi_{q} \Delta_{j} \widetilde{\phi}_{q^{\prime}}\right)\left(\left(\phi_{q^{\prime}} S_{j^{\prime}-1} u\right)\left(\Delta_{j^{\prime}} v\right)(t, \cdot)\right)\right\|_{L^{2}(d x)} \\
& \quad \leqslant C_{N} \sum_{q^{\prime}}\left\langle q-q^{\prime}\right\rangle^{-N}\left\|\phi_{q^{\prime}} S_{j^{\prime}-1} u(t, \cdot)\right\|_{L^{\infty}}\left\|\Delta_{j^{\prime}} v(t, \cdot)\right\|_{L^{2}(d x)} .
\end{aligned}
$$

and we apply again lemma 3.1.4.

We will now prove an estimate for the remainder.

Proposition 3.1.5

(i) Let $\left(s_{1}, s_{1}^{\prime}, \alpha_{1}, \alpha_{1}^{\prime}, r_{1}\right),\left(s_{2}, s_{2}^{\prime}, \alpha_{2}, \alpha_{2}^{\prime}, r_{2}\right)$ be real numbers satisfying

$$
\begin{aligned}
& s_{1}+s_{2}+s_{1}^{\prime}+s_{2}^{\prime}>0, \quad s_{1}+s_{2}>\alpha_{1}^{\prime}+\alpha_{2}^{\prime} \\
& \alpha_{1}+\alpha_{2}+\alpha_{1}^{\prime}+\alpha_{2}^{\prime}<0, \quad r_{j} \geqslant 1, j=1,2 .
\end{aligned}
$$


Let $u \in F_{\alpha_{1}, \alpha_{1}^{\prime}, r_{1}}^{s_{1}, s^{\prime}}$ (resp. $u \in \underline{H}_{\alpha_{1}, \alpha_{1}^{\prime}}^{s_{1}, s_{1}^{\prime}}$ ) and $v \in H_{\alpha_{2}, \alpha_{2}^{\prime}, r_{2}}^{s_{2}, s_{2}^{\prime}}$. Then $R(u, v)$ belongs to $F_{\alpha, \alpha^{\prime}, r}^{s, s^{\prime}}\left(\right.$ resp. to $\left.H_{\alpha, \alpha^{\prime}, r_{2}}^{s, s^{\prime}}\right)$ with

$$
\begin{array}{r}
s=s_{1}+s_{2}-d, \quad s^{\prime}=s_{1}^{\prime}+s_{2}^{\prime}+\frac{d}{2} \\
\alpha=\alpha_{1}+\alpha_{2}+d, \quad \alpha^{\prime}=\alpha_{1}^{\prime}+\alpha_{2}^{\prime}-\frac{d}{2} \\
\frac{1}{r}=\min \left[1, \frac{1}{r_{1}}+\frac{1}{r_{2}}\right] .
\end{array}
$$

(ii) Let us assume instead of (3.1.23)

$$
\begin{aligned}
& s_{1}+s_{2}+s_{1}^{\prime}+s_{2}^{\prime}>0, \quad s_{1}+s_{2}>\alpha_{1}^{\prime}+\alpha_{2}^{\prime} \\
& \alpha_{1}+\alpha_{1}^{\prime}<0, \quad \alpha_{2}^{\prime}>\frac{d}{2}, \quad r_{j} \geqslant 1, j=1,2
\end{aligned}
$$

and suppose that $v \in \widetilde{H}_{\alpha_{2}, \alpha_{2}^{\prime}, r_{2}}^{s_{2}, s_{2}^{\prime}}$. We then have $R(u, v) \in F_{\alpha_{1}, \alpha_{1}^{\prime}, r}^{s, s^{\prime}}$ (resp. $R(u, v) \in$ $H_{\alpha_{1}, \alpha_{1}^{\prime}, r_{2}}^{s,{ }^{\prime}}$ ) for the values of $s, s^{\prime}, r$ given by (3.1.24).

Proof. — We will estimate for $u \in F_{\alpha_{1}, \alpha_{1}^{\prime}, r_{1}}^{s_{1}, s_{1}^{\prime}}$

$$
\phi_{q} \Delta_{j} R(u, v)=\sum_{\substack{k, k^{\prime} \\\left|k-k^{\prime}\right| \leqslant 1 \\ k \geqslant j-N_{0}}} \sum_{q^{\prime}}\left(\phi_{q} \Delta_{j} \widetilde{\phi}_{q^{\prime}}\right)\left[\left(\widetilde{\phi}_{q^{\prime}} \Delta_{k} u\right)\left(\phi_{q^{\prime}} \Delta_{k^{\prime}} v\right)\right]
$$

where $N_{0}$ is a large enough integer. At fixed $t$, the $L^{2}(d x)$ norm of the general term of (3.1.26) is bounded from above by

$$
C_{N}\left\langle q-q^{\prime}\right\rangle^{-N} 2^{j d / 2}\left\|\widetilde{\phi}_{q^{\prime}} \Delta_{k} u(t, \cdot)\right\|_{L^{2}(d x)}\left\|\phi_{q^{\prime}} \Delta_{k^{\prime}} v(t, \cdot)\right\|_{L^{2}(d x)} \cdot
$$

We multiply this expression by $\Gamma_{\ell}(t)$ and compute the $L^{2}(d t / t)$ norm in function of $\left\|\widetilde{\phi}_{q^{\prime}} \Delta_{k} u \Gamma_{\ell}(t)\right\|_{L^{2}\left(\frac{d t}{t} d x\right)}$ and of $\left\|\phi_{q^{\prime}} \Delta_{k^{\prime}} v \Gamma_{\ell}(t)\right\|_{L^{\infty}\left(d t, L^{2}(d x)\right)}$. By definition of the spaces, we get an upper bound given by an expression $C_{N}\left\langle q-q^{\prime}\right\rangle^{-N} d_{j k \ell q^{\prime}}$ with

$$
\begin{aligned}
d_{j k \ell q^{\prime}}=2^{j d / 2} 2^{(k-\ell / 2)\left(\alpha_{1}+\alpha_{2}\right)}( & \left.+2^{k-\ell / 2}\right)^{-s_{1}-s_{2}-\alpha_{1}-\alpha_{2}} \\
& \times 2^{(k-\ell)\left(\alpha_{1}^{\prime}+\alpha_{2}^{\prime}\right)}\left(1+2^{k-\ell}\right)^{-s_{1}^{\prime}-s_{2}^{\prime}-\alpha_{1}^{\prime}-\alpha_{2}^{\prime}} c_{k q^{\prime}}
\end{aligned}
$$

with $\left(c_{k q^{\prime}}\right)_{k q^{\prime}} \in \ell_{k}^{1} \ell_{q^{\prime}}^{r}$, where we used that $\left|k-k^{\prime}\right| \leqslant 1$. We must show that

$$
\sum_{k \geqslant j-N_{0}} d_{j k \ell q^{\prime}} \leqslant C 2^{(j-\ell / 2) \widetilde{\alpha}}\left(1+2^{j-\ell / 2}\right)^{-s-\widetilde{\alpha}} 2^{(j-\ell) \widetilde{\alpha}^{\prime}}\left(1+2^{j-\ell}\right)^{-s^{\prime}-\widetilde{\alpha}^{\prime}} c_{j q^{\prime}}^{\prime}
$$

with $\left(c_{j q^{\prime}}^{\prime}\right)_{j q^{\prime}}$ in the unit ball of $\ell_{j}^{1} \ell_{q^{\prime}}^{r} \subset \ell_{j}^{2} \ell_{q^{\prime}}^{r}$, and where $\left(\widetilde{\alpha}, \widetilde{\alpha}^{\prime}\right)=\left(\alpha, \alpha^{\prime}\right)$ in case (i), and $\left(\widetilde{\alpha}, \widetilde{\alpha}^{\prime}\right)=\left(\alpha_{1}, \alpha_{1}^{\prime}\right)$ in case (ii). 
Case $j \geqslant \ell$. We have

$$
\begin{aligned}
\sum_{k \geqslant j-N_{0}} d_{j k \ell q^{\prime}} & \leqslant C \sum_{k \geqslant j-N_{0}} 2^{j d / 2} 2^{-k\left(s_{1}+s_{2}+s_{1}^{\prime}+s_{2}^{\prime}\right)} 2^{\ell\left(\frac{s_{1}+s_{2}}{2}+s_{1}^{\prime}+s_{2}^{\prime}\right)} c_{k q^{\prime}} \\
& \leqslant C 2^{-(j-\ell / 2) s-(j-\ell) s^{\prime}} c_{j q^{\prime}}^{\prime}
\end{aligned}
$$

with $\left(c_{j q^{\prime}}^{\prime}\right)_{j q^{\prime}} \in \ell_{j}^{1} \ell_{q^{\prime}}^{r}$ because of assumptions (3.1.23) or (3.1.25), $s$ and $s^{\prime}$ being given by (3.1.24). This gives (3.1.29) both in cases (i) and (ii).

Case $\ell>j \geqslant \ell / 2$. - We have

$$
\begin{aligned}
\sum_{k \geqslant j-N_{0}} d_{j k \ell q^{\prime}} \leqslant & C 2^{j \frac{d}{2}-\frac{\ell}{2}\left(s_{1}+s_{2}\right)} c_{\ell q^{\prime}}^{\prime} \\
& +\sum_{k ; \ell>k>j-N_{0}} 2^{j d / 2} 2^{-k\left(s_{1}+s_{2}-\alpha_{1}^{\prime}-\alpha_{2}^{\prime}\right)} 2^{\ell\left(\frac{s_{1}+s_{2}}{2}-\alpha_{1}^{\prime}-\alpha_{2}^{\prime}\right)} c_{k q^{\prime}}
\end{aligned}
$$

where the first contribution is given by the sum (3.1.30) taken for $k \geqslant \ell$ instead of $k \geqslant j-N_{0}$, and where $\left(c_{\ell q^{\prime}}^{\prime}\right)_{\ell q^{\prime}} \in \ell_{\ell}^{1} \ell_{q^{\prime}}^{r}$. The right hand side of (3.1.31) is smaller than

$$
C 2^{-(j-\ell / 2)\left(s_{1}+s_{2}-d\right)} 2^{(j-\ell)\left(\alpha_{1}^{\prime}+\alpha_{2}^{\prime}-d / 2\right)} \times\left[2^{(j-\ell)\left(s_{1}+s_{2}-\alpha_{1}^{\prime}-\alpha_{2}^{\prime}\right)} c_{\ell q^{\prime}}^{\prime}+c_{j q^{\prime}}\right]
$$

because of (3.1.23), (3.1.25). The sequence between brackets is smaller than a $\ell_{j}^{1} \ell_{q^{\prime}}^{r}$ sequence independent of $\ell$. This gives the estimate (3.1.29) in cases (i) and (ii), remembering for the last one that $\alpha_{2}^{\prime}>d / 2$.

Case $\ell / 2>j$. - We first treat case (i). We have

$$
\begin{aligned}
\sum_{k \geqslant j-N_{0}} d_{j k \ell q^{\prime}} & \leqslant C\left[2^{j \frac{d}{2}-\frac{\ell}{2}\left(s_{1}+s_{2}\right)} c_{\ell q^{\prime}}^{\prime}+2^{j \frac{d}{2}-\frac{\ell}{2}\left(\alpha_{1}^{\prime}+\alpha_{2}^{\prime}\right)} c_{\ell q^{\prime}}^{\prime \prime}\right] \\
& +\sum_{k ; \ell / 2>k>j-N_{0}} 2^{j d / 2} 2^{k\left(\alpha_{1}+\alpha_{2}+\alpha_{1}^{\prime}+\alpha_{2}^{\prime}\right)} 2^{-\frac{\ell}{2}\left(\alpha_{1}+\alpha_{2}+2\left(\alpha_{1}^{\prime}+\alpha_{2}^{\prime}\right)\right)} c_{k q^{\prime}}
\end{aligned}
$$

where the first two terms are given by the sum (3.1.31) taken for $k \geqslant \ell / 2$ instead of $k \geqslant j-N_{0}$, and where $\left(c_{\ell q^{\prime}}^{\prime}\right)_{\ell q^{\prime}},\left(c_{\ell q^{\prime}}^{\prime \prime}\right)_{\ell q^{\prime}}$ are in $\ell_{\ell}^{1} \ell_{q^{\prime}}^{r}$. Using (3.1.23) we get for the right hand side of (3.1.32) the estimate

$$
\begin{aligned}
& C 2^{(j-\ell / 2)\left(\alpha_{1}+\alpha_{2}+d\right)} 2^{(j-\ell)\left(\alpha_{1}^{\prime}+\alpha_{2}^{\prime}-d / 2\right)} \\
& \times\left[2^{-(j-\ell / 2)\left(\alpha_{1}+\alpha_{2}+\alpha_{1}^{\prime}+\alpha_{2}^{\prime}\right)} 2^{-\frac{\ell}{2}\left(s_{1}+s_{2}-\alpha_{1}^{\prime}-\alpha_{2}^{\prime}\right)} c_{\ell q^{\prime}}^{\prime}\right. \\
&\left.+2^{-(j-\ell / 2)\left(\alpha_{1}+\alpha_{2}+\alpha_{1}^{\prime}+\alpha_{2}^{\prime}\right)} c_{\ell q^{\prime}}^{\prime \prime}+\widetilde{c}_{j q^{\prime}}\right]
\end{aligned}
$$

with $\left(\widetilde{c}_{j q^{\prime}}\right)_{j q^{\prime}} \in \ell_{j}^{1} \ell_{q^{\prime}}^{r}$. The term between brackets is smaller than a sequence indexed by $\left(j, q^{\prime}\right)$, independent of $\ell$, and belonging to $\ell_{j}^{1} \ell_{q^{\prime}}^{r}$. This gives conclusion (3.1.29) in case (i). In case (ii), we estimate the $L^{2}(d x)$ norm of the general term of (3.1.26) 
using (3.1.27) when $k \geqslant \ell / 2$, and writing

$$
\begin{aligned}
\left\|\left(\phi_{q} \Delta_{j} \widetilde{\phi}_{q^{\prime}}\right)\left[\left(\widetilde{\phi}_{q^{\prime}} \Delta_{k} u\right)\left(\phi_{q^{\prime}} \Delta_{k^{\prime}} v\right)\right](t, \cdot)\right\|_{L^{2}} \\
\quad \leqslant C\left\langle q-q^{\prime}\right\rangle^{-N}\left\|\widetilde{\phi}_{q^{\prime}} \Delta_{k} u(t, \cdot)\right\|_{L^{2}}\left\|\phi_{q^{\prime}} \Delta_{k^{\prime}} v(t, \cdot)\right\|_{L^{\infty}}
\end{aligned}
$$

when $\ell / 2>k \geqslant j-N_{0}$. The assumption $\left(\left\|\phi_{q} \chi(D / \sqrt{t}) v\right\|_{L^{\infty}}\right)_{q} \in \ell_{q}^{r_{2}}$ implies that there is $\left(c_{q^{\prime}}\right)_{q^{\prime}} \in \ell_{q^{\prime}}^{r_{2}}$ such that for any $k^{\prime}$ with $k^{\prime} \leqslant \ell / 2+N_{1},\left\|\phi_{q^{\prime}} \Delta_{k^{\prime}} v\right\|_{L^{\infty}} \leqslant c_{q^{\prime}}$. Consequently, if we multiply (3.1.34) by $\Gamma_{\ell}(t)$ and compute the $L^{2}(d t / t)$ norm, we get an upper bound of form $C_{N}\left\langle q-q^{\prime}\right\rangle^{-N} e_{k \ell q^{\prime}}$ with

$$
e_{k \ell q^{\prime}}=2^{(k-\ell / 2) \alpha_{1}} 2^{(k-\ell) \alpha_{1}^{\prime}} c_{k q^{\prime}}
$$

with $\left(c_{k q^{\prime}}\right)_{k q^{\prime}} \in \ell_{k}^{2} \ell_{q^{\prime}}^{r}$. To finish the proof, we must show that

$$
\sum_{k ; \ell / 2>k \geqslant j-N_{0}} e_{k \ell q^{\prime}}+\sum_{k \geqslant \ell / 2} d_{j k \ell q^{\prime}}
$$

is smaller than the right hand side of (3.1.29) with $\widetilde{\alpha}=\alpha_{1}, \widetilde{\alpha}^{\prime}=\alpha_{1}^{\prime}$. The contribution of the second sum in (3.1.35) is smaller than the first two contributions in the right hand side of (3.1.32) i.e. to

$$
C 2^{(j-\ell / 2) \alpha_{1}} 2^{(j-\ell) \alpha_{1}^{\prime}}\left[2^{j\left(d / 2-\alpha_{2}^{\prime}\right)} 2^{(j-\ell / 2)\left(\alpha_{2}^{\prime}-\alpha_{1}-\alpha_{1}^{\prime}\right)} \widetilde{c}_{\ell q^{\prime}}\right]
$$

with $\left(\widetilde{c}_{\ell q^{\prime}}\right)_{\ell q^{\prime}} \in \ell_{\ell}^{1} \ell_{q^{\prime}}^{r}$, and the term between brackets is smaller than a sequence in $\ell_{j}^{1} \ell_{q^{\prime}}^{r}$, because of the assumption (3.1.25) $\alpha_{2}^{\prime}>d / 2, \alpha_{1}+\alpha_{1}^{\prime}<0$. The first sum in (3.1.35) is smaller than

$$
C 2^{(j-\ell / 2) \alpha_{1}} 2^{(j-\ell) \alpha_{1}^{\prime}}\left[\sum_{k ; \ell / 2>k \geqslant j-N_{0}} 2^{(k-j)\left(\alpha_{1}+\alpha_{1}^{\prime}\right)} c_{k q^{\prime}}\right]
$$

and the term between brackets is a $\ell_{j}^{2} \ell_{q^{\prime}}^{r}$ sequence since $\alpha_{1}+\alpha_{1}^{\prime}<0$. This concludes the proof in the case $u \in F_{\alpha_{1}, \alpha_{1}^{\prime}, r_{1}}^{s_{1}, s_{1}^{\prime}}$.

To prove (i) and (ii) for $u \in \underline{H}_{\alpha_{1}, \alpha_{1}^{\prime}}^{s_{1}, s^{\prime}}$ we write instead of (3.1.26)

$$
\phi_{q} \Delta_{j} R(u, v)=\sum_{\substack{k, k^{\prime} \\\left|k-k^{\prime}\right| \leqslant 1 \\ k \geqslant j-N_{0}}} \sum_{q^{\prime}}\left(\phi_{q} \Delta_{j} \widetilde{\phi}_{q^{\prime}}\right)\left[\left(\Delta_{k} u\right)\left(\phi_{q^{\prime}} \Delta_{k^{\prime}} v\right)\right] .
$$

The $L^{2}(d x)$ norm of the general term of the above sum is smaller than

$$
C_{N}\left\langle q-q^{\prime}\right\rangle^{-N} 2^{j d / 2}\left\|\Delta_{k} u(t, \cdot)\right\|_{L^{2}(d x)}\left\|\phi_{q^{\prime}} \Delta_{k^{\prime}} v(t, \cdot)\right\|_{L^{2}(d x)} .
$$

We multiply this expression by $\Gamma_{\ell}(t)$ and compute the $L^{\infty}(d t)$ norm in function of $\left\|\Delta_{k} u(t, \cdot)\right\|_{L^{\infty}\left(d t, L^{2}(d x)\right)}$ and of $\left\|\phi_{q^{\prime}} \Delta_{k^{\prime}} v(t, \cdot) \Gamma_{\ell}(t)\right\|_{L^{\infty}\left(d t, L^{2}(d x)\right)}$. We get an upper bound given by (3.1.28) with a sequence $\left(c_{k q^{\prime}}\right)_{k q^{\prime}}$ in $\ell_{k}^{1} \ell_{q^{\prime}}^{r_{2}}$ instead of $\ell_{k}^{1} \ell_{q^{\prime}}^{r}$. The rest of the proof is then identical as above.

We will also need: 
Proposition 3.1.6

(i) Let $\kappa_{1} \in \mathbb{N}, \kappa_{2} \in \mathbb{N}^{d}$, s, $s^{\prime}, \alpha, \alpha^{\prime}$ be real numbers satisfying

$$
\begin{aligned}
& s+s^{\prime}>2 \kappa_{1}+\left|\kappa_{2}\right|, \quad s>2 \kappa_{1}+\alpha^{\prime} \\
& s>2 \kappa_{1}+\alpha+2 \alpha^{\prime}, \quad \alpha+\alpha^{\prime}<d / 2 .
\end{aligned}
$$

Let $m \in \mathbb{Z}^{*}, r \in[1,+\infty]$, and $w \in F_{\alpha, \alpha^{\prime}, r}^{s, s^{\prime}}$ (resp. $w \in H_{\alpha, \alpha^{\prime}, r}^{s, s^{\prime}}$ ). Then

$$
R\left(w,\left(t D_{t}\right)^{\kappa_{1}}\left(D_{x} / t\right)^{\kappa_{2}} e^{i m \theta}\right) \in F_{\alpha, \alpha^{\prime}, r}^{s-\kappa_{1}, s^{\prime}-\kappa_{2}}
$$

(resp.

$$
\left.R\left(w,\left(t D_{t}\right)^{\kappa_{1}}\left(D_{x} / t\right)^{\kappa_{2}} e^{i m \theta}\right) \in H_{\alpha, \alpha^{\prime}, r}^{s-\kappa_{1}, s^{\prime}-\kappa_{2}}\right) .
$$

(ii) Let $\kappa_{1} \in\{0,1\},\left|\kappa_{2}\right|=1, s, s^{\prime}, \alpha, \alpha^{\prime}$ be real numbers satisfying

$$
\begin{gathered}
s+s^{\prime}>2 \kappa_{1}+1, \quad s>2 \kappa_{1}+\alpha^{\prime}, \quad s>2 \kappa_{1}-2+2\left(\alpha+2 \alpha^{\prime}+2\right) \\
-\frac{d}{2}-1<\alpha+\alpha^{\prime}, \quad 2 \alpha+3 \alpha^{\prime}+1<\frac{d}{2} .
\end{gathered}
$$

Then if $\beta=2\left(\alpha+\alpha^{\prime}+1\right), w \in F_{\alpha^{\prime}, \alpha^{\prime}, r}^{s, s^{\prime}}\left(\right.$ resp. $\left.w \in H_{\alpha^{\prime}, \alpha^{\prime}, r}^{s, s^{\prime}}\right)$ one has

$$
R\left(w,\left(t D_{t}\right)^{\kappa_{1}}\left(D_{x} / t\right)^{\kappa_{2}} e^{i m \theta}\right) \in F_{\beta, \alpha^{\prime}, r}^{s-2 \kappa_{1}, s^{\prime}-1}
$$

(resp.

$$
\left.R\left(w,\left(t D_{t}\right)^{\kappa_{1}}\left(D_{x} / t\right)^{\kappa_{2}} e^{i m \theta}\right) \in H_{\beta, \alpha^{\prime}, r}^{s-2 \kappa_{1}, s^{\prime}-1}\right) .
$$

The same results hold true for $\widetilde{R}$ instead of $R$.

Proof. - We write with $f=\left(t D_{t}\right)^{\kappa_{1}}\left(D_{x} / t\right)^{\kappa_{2}} e^{i m \theta}$

$$
\phi_{q} \Delta_{j} R(w, f)(t, \cdot)=\sum_{\substack{k, k^{\prime} \\\left|k-k^{\prime}\right| \leqslant 1}} \sum_{q^{\prime}}\left(\phi_{q} \Delta_{j} \widetilde{\phi}_{q^{\prime}}\right)\left(\left(\phi_{q^{\prime}} \Delta_{k} w\right)\left(\Delta_{k^{\prime}} f\right)\right) .
$$

We first treat case (i). Remark that since

we have

$$
t D_{t}\left(e^{i m \theta}\right)=m \frac{t x^{2}}{4} e^{i m \theta}=\frac{1}{m}\left[\frac{D_{x}^{2}}{t}+i m\right] e^{i m \theta}
$$

$$
\begin{aligned}
&\left\|\Delta_{k}\left(\left(t D_{t}\right)^{\kappa_{1}}\left(\frac{D_{x}}{t}\right)^{\kappa_{2}} e^{i m \theta}\right)\right\|_{L^{\infty}(d x)} \leqslant C\left(1+\frac{2^{k}}{\sqrt{t}}\right)^{2 \kappa_{1}}\left(\frac{2^{k}}{t}\right)^{\left|\kappa_{2}\right|} \\
&\left\|\Delta_{k}\left(t D_{t}\right)^{\kappa_{1}}\left(\frac{D_{x}}{t}\right)^{\kappa_{2}} e^{i m \theta}\right\|_{L^{2}(d x)} \leqslant C\left(\frac{2^{k}}{t}\right)^{d / 2}\left(1+\frac{2^{k}}{\sqrt{t}}\right)^{2 \kappa_{1}}\left(\frac{2^{k}}{t}\right)^{\left|\kappa_{2}\right|} .
\end{aligned}
$$

This shows in particular that it is enough to prove (3.1.37), (3.1.38) in the special case $\kappa_{1}=\kappa_{2}=0$, to which one can reduce changing the values of $s, s^{\prime}$. We deduce from (3.1.42) that for $p=2$ (resp. $p=\infty)$

$$
\left\|\phi_{q} \Delta_{j} R(w, f) \Gamma_{\ell}(t)\right\|_{L^{p}\left(\frac{d t}{t}, L^{2}(d x)\right)} \leqslant C \sum_{q^{\prime}}\left\langle q-q^{\prime}\right\rangle^{-N} d_{j \ell q^{\prime}}
$$


with

$$
\begin{aligned}
d_{j \ell q^{\prime}}= & \sum_{\substack{k, k^{\prime} \\
j-N_{0} \leqslant k<\ell-j \\
\left|k-k^{\prime}\right| \leqslant 1}} 2^{j \frac{d}{2}}\left\|\phi_{q^{\prime}} \Gamma_{\ell}(t) \Delta_{k} w\right\|_{L^{p}\left(\frac{d t}{t}, L^{2}(d x)\right)}\left\|\Delta_{k^{\prime}} f \Gamma_{\ell}(t)\right\|_{L^{\infty}\left(L^{2}\right)} \\
& +\sum_{\substack{k, k^{\prime} \\
k \geqslant j-N_{0} \\
k \geqslant \ell-j \\
k-k^{\prime} \mid \leqslant 1}}\left\|\phi_{q^{\prime}} \Gamma_{\ell}(t) \Delta_{k} w\right\|_{L^{p}\left(\frac{d t}{t}, L^{2}(d x)\right)}\left\|\Delta_{k^{\prime}} f \Gamma_{\ell}(t)\right\|_{L^{\infty}(d t d x)} .
\end{aligned}
$$

We estimate (3.1.45) in the following cases:

Case $j \geqslant \ell$. - In this case, only the second sum in (3.1.45) remains. It is bounded from above by

$$
\sum_{k \geqslant j-N_{0}} 2^{-(k-\ell / 2) s-(k-\ell) s^{\prime}} c_{k q^{\prime}} \leqslant C 2^{-(j-\ell / 2) s-(j-\ell) s^{\prime}} c_{j q^{\prime}}^{\prime}
$$

for $\ell^{2} \ell^{r}$ sequences $\left(c_{k q^{\prime}}\right)_{k q^{\prime}},\left(c_{j q^{\prime}}^{\prime}\right)_{j q^{\prime}}$, since $s+s^{\prime}>0$. This gives the needed conclusion. Case $\ell>j \geqslant \ell / 2$. - Again, only the second sum of (3.1.45) contributes. We get the upper bound

$$
\begin{aligned}
& \sum_{k=j-N_{0}}^{\ell} 2^{-(k-\ell / 2) s+(k-\ell) \alpha^{\prime}} c_{k q^{\prime}}+2^{-\ell s / 2} c_{\ell q^{\prime}}^{\prime} \\
& =2^{-(j-\ell / 2) s+(j-\ell) \alpha^{\prime}}\left[\sum_{k=j-N_{0}}^{\ell} 2^{-(k-j)\left(s-\alpha^{\prime}\right)} c_{k q^{\prime}}+2^{(j-\ell)\left(s-\alpha^{\prime}\right)} c_{\ell q^{\prime}}^{\prime}\right]
\end{aligned}
$$

for $\ell^{2} \ell^{r}$ sequences $\left(c_{k q^{\prime}}\right)_{k q^{\prime}},\left(c_{\ell q^{\prime}}^{\prime}\right)_{\ell q^{\prime}}$, and since $s>\alpha^{\prime}$, the expression between brackets is a $\ell_{j}^{2} \ell_{q^{\prime}}^{r}$ sequence, which gives the result.

Case $\ell / 2>j$. - The first sum in (3.1.45) is bounded from above, using (3.1.43), by

$$
\begin{aligned}
& \sum_{k=j-N_{0}}^{\ell / 2} 2^{j d / 2} 2^{(k-\ell / 2) \alpha+(k-\ell)\left(\alpha^{\prime}+d / 2\right)} c_{k q^{\prime}}+\sum_{k=\ell / 2}^{\ell-j} 2^{j d / 2} 2^{-(k-\ell / 2) s+(k-\ell)\left(\alpha^{\prime}+d / 2\right)} c_{k q^{\prime}} \\
& =2^{(j-\ell / 2) \alpha+(j-\ell) \alpha^{\prime}}\left[\sum_{k=j-N_{0}}^{\ell / 2} 2^{(j-k)\left(d / 2-\alpha-\alpha^{\prime}\right)+(k-\ell / 2) d} c_{k q^{\prime}}\right. \\
& \left.+\sum_{k=\ell / 2}^{\ell-j} 2^{(j+k-\ell)\left(d / 2-\alpha-\alpha^{\prime}\right)+(k-\ell / 2)\left(\alpha+2 \alpha^{\prime}-s\right)} c_{k q^{\prime}}\right]
\end{aligned}
$$

and since $d / 2>\alpha+\alpha^{\prime}, s>\alpha+2 \alpha^{\prime}$, the term between brackets is smaller than a $\ell_{j}^{2} \ell_{q^{\prime}}^{r}$ sequence independent of $\ell$. This gives the wanted estimate. We still have to study 
the contribution to the second sum in (3.1.45). We get an upper bound

$$
\begin{gathered}
C 2^{(j-\ell / 2) \alpha+(j-\ell) \alpha^{\prime}}\left[\sum_{k=\ell-j}^{\ell} 2^{-(j+k-\ell)\left(s-\alpha^{\prime}\right)+(j-\ell / 2)\left(s-\alpha-2 \alpha^{\prime}\right)} c_{k q^{\prime}}\right. \\
\left.\quad+\sum_{k>\ell} 2^{-(k-\ell)\left(s+s^{\prime}\right)+(j-\ell / 2)\left(s-\alpha-2 \alpha^{\prime}\right)-j\left(s-\alpha^{\prime}\right)} c_{k q^{\prime}}\right] .
\end{gathered}
$$

Since $s>\alpha^{\prime}, s>\alpha+2 \alpha^{\prime}, s+s^{\prime}>0$, the term between brackets is in $\ell_{j}^{2} \ell_{q^{\prime}}^{r}$. This concludes the proof of the proposition in case (i).

To treat case (ii), let us remark first that assumption (3.1.39) implies the first two relations (3.1.36). Moreover, conclusions (3.1.40), (3.1.41) coincide with (3.1.37), (3.1.38) when the frequency $j$ in the left hand side of (3.1.44) satisfies $j \geqslant \ell / 2$. Since, in case (i), the estimates of (3.1.44) when $j \geqslant \ell / 2$ used only the first two relations (3.1.36), we see that we just need to study the case $j<\ell / 2$. We can also reduce to $\kappa_{1}=0$, replacing $s$ by $s-2 \kappa_{1}$. Using (3.1.43) with $\left|\kappa_{2}\right|=1$, we estimate the first sum in (3.1.45) by

$$
\begin{aligned}
2^{(j-\ell / 2) \beta+(j-\ell) \alpha^{\prime}}\left[\sum_{k=j}^{\ell / 2} 2^{(k-\ell / 2)\left(\alpha+\alpha^{\prime}+\frac{d}{2}+1\right)} 2^{-(j-\ell / 2)\left(2 \alpha+3 \alpha^{\prime}+1-\frac{d}{2}\right)} 2^{-j} c_{k q^{\prime}}\right. \\
\\
\left.\quad+\sum_{k=\ell / 2}^{\ell-j} 2^{\left(\alpha^{\prime}+\frac{d}{2}+1-s\right)(k+j-\ell)} 2^{(j-\ell / 2)\left(s+2-2\left(\alpha+2 \alpha^{\prime}+2\right)\right)} 2^{-j} c_{k q^{\prime}}\right] .
\end{aligned}
$$

Since by (3.1.39), $\alpha+\alpha^{\prime}+\frac{d}{2}+1>0$ and $2 \alpha+3 \alpha^{\prime}+1-\frac{d}{2}<0$, the first sum inside the bracket is a $\ell_{j}^{2} \ell_{q^{\prime}}^{r}$ sequence. When the coefficient of $(k+j-\ell)$ in the second sum is strictly positive, we also get that this sum is a $\ell_{j}^{2} \ell_{q^{\prime}}^{r}$ sequence since, by (3.1.39), $s>-2+2\left(\alpha+2 \alpha^{\prime}+2\right)$. When the coefficient of $(k+j-\ell)$ is non-positive, we get for this sum an upper bound

$$
C 2^{(j-\ell / 2)\left(\frac{d}{2}-1-2 \alpha-3 \alpha^{\prime}-\delta\right)} 2^{-j} c_{\ell q^{\prime}}^{\prime}
$$

with $\left(c_{\ell q^{\prime}}^{\prime}\right)_{\ell q^{\prime}} \in \ell_{\ell}^{2} \ell_{q^{\prime}}^{r}, \delta>0$ when the coefficient of $k+j-\ell$ is zero, $\delta=0$ otherwise. Since $\frac{d}{2}-1-2 \alpha-3 \alpha^{\prime}>0$, we see that the supremum in $\ell>2 j$ of (3.1.46) is in $\ell_{j}^{2} \ell_{q^{\prime}}^{r}$.

Let us estimate now the second sum in (3.1.45): using (3.1.43), we get the estimate

$$
\begin{gathered}
2^{(j-\ell / 2) \beta+(j-\ell) \alpha^{\prime}}\left[\sum_{k=\ell-j}^{\ell} 2^{-(k-\ell)\left(s-1-\alpha^{\prime}\right)} 2^{-(j-\ell / 2)\left(2\left(\alpha+2 \alpha^{\prime}+2\right)-s-2\right)} 2^{-j\left(s-\alpha^{\prime}\right)} c_{k q^{\prime}}\right. \\
\left.\quad+\sum_{k=\ell}^{+\infty} 2^{-(k-\ell)\left(s+s^{\prime}-1\right)} 2^{(j-\ell / 2)\left(s+2-2\left(\alpha+2 \alpha^{\prime}+2\right)\right)} 2^{-j\left(s-\alpha^{\prime}\right)} c_{k q^{\prime}}\right] .
\end{gathered}
$$

When $s-1-\alpha^{\prime}>0$, the first sum is smaller than

$$
2^{-(j-\ell / 2)\left(2\left(\alpha+2 \alpha^{\prime}+2\right)-s-2\right)} 2^{-j} c_{\ell-j, q^{\prime}}^{\prime}
$$


with $\left(c_{\ell q^{\prime}}^{\prime}\right)_{\ell q^{\prime}} \in \ell_{\ell}^{2} \ell_{q^{\prime}}^{r}$, so smaller than a $\ell_{j}^{2} \ell_{q^{\prime}}^{r}$ sequence using assumption (3.1.39) and $j \leqslant \ell / 2$. When $s-1-\alpha^{\prime} \leqslant 0$, we get an upper bound

$$
2^{(j-\ell / 2)\left(s+2-2\left(\alpha+2 \alpha^{\prime}+2\right)\right)} 2^{-j\left(s-\alpha^{\prime}\right)+j \delta} c_{\ell q^{\prime}},
$$

with $\delta>0$ arbitrarily small, which gives again a $\ell_{j}^{2} \ell_{q^{\prime}}^{r}$ upper bound using $s>\alpha^{\prime}$. For the second sum, we have $s+s^{\prime}>1$, whence again an upper bound in terms of a $\ell_{j}^{2} \ell_{q^{\prime}}^{r}$ sequence.

We want to deduce from the preceding results a statement concerning products. Let us set the following definition.

Definition 3.1.7. - Let $s, s^{\prime}, \alpha, \alpha^{\prime}$ be real numbers, $r \in[1,+\infty], M \in \mathbb{N}$. Denote by $\frac{1}{\widetilde{r}}=\min \left[1, \frac{1}{r}+\frac{1}{2}\right]$. We set

$$
\begin{aligned}
E_{\alpha, \alpha^{\prime}, r}^{s, s^{\prime}} & =H_{\alpha, \alpha^{\prime}, \widetilde{r}}^{s, s^{\prime}} \cap F_{\alpha, \alpha^{\prime}, r}^{s, s^{\prime}+1 / 2}, \quad \underline{E}_{\alpha, \alpha^{\prime}, r}^{s, s^{\prime}}=\underline{H}_{\alpha, \alpha^{\prime}}^{s, s^{\prime}} \cap F_{\alpha, \alpha^{\prime}, r}^{s, s^{\prime}+1 / 2} \\
\widetilde{E}_{\alpha, \alpha^{\prime}, r}^{s, s^{\prime}} & =\widetilde{H}_{\alpha, \alpha^{\prime}, \widetilde{r}}^{s, s^{\prime}} \cap F_{\alpha, \alpha^{\prime}, r}^{s, s^{\prime}+1 / 2}, \quad \underline{\widetilde{E}}_{\alpha, \alpha^{\prime}, r}^{s, s^{\prime}}=\underline{\widetilde{H}}_{\alpha, \alpha^{\prime}}^{s, s^{\prime}} \cap F_{\alpha, \alpha^{\prime}, r}^{s, s^{\prime}+1 / 2} \\
\mathcal{E}_{\alpha, \alpha^{\prime}, r}^{s, s^{\prime}}(M) & =\left\{u \in E_{\alpha, \alpha^{\prime}, r}^{s, s^{\prime}} ; \forall \gamma \in \mathbb{N}^{d},|\gamma| \leqslant M, x^{\gamma} u \in E_{\alpha, \alpha^{\prime}, r}^{s, s^{\prime}-|\gamma|}\right\} \\
\mathcal{E}_{\alpha, \alpha^{\prime}, r}^{s, s^{\prime}}(M) & =\left\{u \in \underline{E}_{\alpha, \alpha^{\prime}, r}^{s, s^{\prime}} ; \forall \gamma \in \mathbb{N}^{d},|\gamma| \leqslant M, x^{\gamma} u \in \underline{E}_{\alpha, \alpha^{\prime}, r}^{s, s^{\prime}|\gamma|}\right\} \\
\widetilde{\mathcal{E}}_{\alpha, \alpha^{\prime}, r}^{s, s^{\prime}}(M) & =\left\{u \in \widetilde{E}_{\alpha, \alpha^{\prime}, r}^{s, s^{\prime}} ; \forall \gamma \in \mathbb{N}^{d},|\gamma| \leqslant M, x^{\gamma} u \in \widetilde{E}_{\alpha, \alpha^{\prime}, r}^{s, s^{\prime}-|\gamma|}\right\} \\
\widetilde{\mathcal{E}}_{\alpha, s^{\prime}, r}^{s, s^{\prime}}(M) & =\left\{u \in \underline{\widetilde{E}}_{\alpha, \alpha^{\prime}, r}^{s, s^{\prime}} ; \forall \gamma \in \mathbb{N}^{d},|\gamma| \leqslant M, x^{\gamma} u \in \underline{\widetilde{E}}_{\alpha, \alpha^{\prime}, r}^{s, s^{\prime}-|\gamma|}\right\} .
\end{aligned}
$$

Corollary 3.1.8. - Let $s, s^{\prime}, \alpha, \alpha^{\prime}, \sigma_{1}, \sigma_{2}, \sigma_{1}^{\prime}, \sigma_{2}^{\prime}$ be real numbers, $M \in \mathbb{N}, \lambda \in \mathbb{R}_{+}$ a parameter, satisfying

$$
\begin{gathered}
\alpha^{\prime}>d / 2, \quad s>\alpha^{\prime}+\frac{\sigma_{1}+\sigma_{2}}{2}, \quad M>s^{\prime}-\lambda+\frac{d}{2} \\
\sigma_{j} \geqslant 0, \quad s-\sigma_{j} \neq d, \quad \sigma_{j}^{\prime} \in[0,1], \quad j=1,2, \quad s \geqslant d .
\end{gathered}
$$

(i) Assume moreover

$$
-\left(2 \alpha^{\prime}+1\right)<\alpha<-\left(\alpha^{\prime}+1\right) .
$$

Then if $\gamma \in \mathbb{N}^{d},|\gamma| \leqslant 2 M-d-1, u_{1} \in \underline{\widetilde{\mathcal{E}}}_{\alpha+2, \alpha^{\prime}, \infty}^{s-\sigma_{1}, s^{\prime}-\sigma_{1}^{\prime}}(M), u_{2} \in \underline{\widetilde{\mathcal{E}}}_{\alpha, \alpha^{\prime}, \infty}^{s-\sigma_{2}, s^{\prime}-\sigma_{2}^{\prime}}(M)$ and if

$$
s+s^{\prime}>\frac{\sigma_{1}+\sigma_{2}}{2}+\frac{\sigma_{1}^{\prime}+\sigma_{2}^{\prime}}{2}+\frac{|\gamma|}{2}+\frac{d}{2}
$$

we have that $x^{\gamma} u_{1} u_{2} \in \widetilde{E}_{\beta, \alpha^{\prime}, 1}^{\widehat{s}, \widehat{s}^{\prime}(\gamma)}$ where

$$
\begin{aligned}
\widehat{s} & =s-\sigma_{1}-\sigma_{2}, \beta=2\left(\alpha+\alpha^{\prime}+1\right) \\
\widehat{s}^{\prime}(\gamma) & =s^{\prime}-\max \left(\sigma_{1}^{\prime}, \sigma_{2}^{\prime}\right)-\left[1+\lambda-s^{\prime}+|\gamma|+\delta+\frac{d}{2}\right]_{+}
\end{aligned}
$$

where $\delta>0$ is arbitrarily small.

(ii) Assume (3.1.50), (3.1.52) and

$$
\alpha+\alpha^{\prime}<0 \text {. }
$$

Then if $u_{1} \in \underline{\widetilde{\mathcal{E}}}_{\alpha, \alpha^{\prime}, \infty}^{s-\sigma_{1}, s^{\prime}-\sigma_{1}^{\prime}}(M), u_{2} \in \underline{\widetilde{\mathcal{E}}}_{\alpha, \alpha^{\prime}, \infty}^{s-\sigma_{2}, s^{\prime}-\sigma_{2}^{\prime}}(M)$, one has $x^{\gamma} u_{1} u_{2} \in \widetilde{E}_{\alpha, \alpha^{\prime}, 1}^{\widehat{s}, \widehat{s}^{\prime}(\gamma)}$. 
We prove first:

LEMMA 3.1.9. - Let $u \in \underline{\widetilde{E}}_{\alpha, \alpha^{\prime}, r}^{s, s^{\prime}}$ with $x^{\gamma} u \in \underline{\widetilde{E}}_{\alpha, \alpha^{\prime}, r}^{s, s^{\prime}-|\gamma|}$ for $|\gamma| \leqslant d+1$. Then for any $\delta>0, u \in \widetilde{E}_{\alpha, \alpha^{\prime}, 1}^{s, s^{\prime}-d-\delta}$.

Proof. - Write for $q \in \mathbb{Z}^{d}, \ell \in \mathbb{N}$

$$
\begin{aligned}
\left\|\phi_{q} \Delta_{j} u \Gamma_{\ell}(t)\right\|_{L^{\infty}\left(d t, L^{2}(d x)\right)} & \leqslant C\langle q\rangle^{-d-1} \sum_{|\gamma| \leqslant d+1}\left\|x^{\gamma} \phi_{q} \Delta_{j} u \Gamma_{\ell}(t)\right\|_{L^{\infty}\left(L^{2}\right)} \\
& \leqslant C\langle q\rangle^{-d-1} \sum_{|\gamma| \leqslant d+1}\left\|\widetilde{\Delta}_{j, \gamma}\left(x^{\gamma} u\right) \Gamma_{\ell}(t)\right\|_{L^{\infty}\left(L^{2}\right)}
\end{aligned}
$$

where $\widetilde{\Delta}_{j, \gamma}$ is a family of Fourier multipliers defined by a cut-off satisfying conditions (3.1.15). If we use (2.2.5) for $u$ and $x^{\gamma} u$ (replacing $s^{\prime}$ by $\left.s^{\prime}-|\gamma|\right)$ in the right hand side of (3.1.55), and then interpolate with (2.2.5), we obtain that $u \in H_{\alpha, \alpha^{\prime}, 1}^{s, s^{\prime}-d}$. The fact that $u \in F_{\alpha, \alpha^{\prime}, 1}^{s, s^{\prime}+\frac{1}{2}-d-\delta}$ is proved in the same way. One proves similarly that $\left\|\phi_{q} \chi(D / \sqrt{t}) u\right\|_{L^{\infty}} \leqslant C\langle q\rangle^{-d-1}$. This concludes the proof.

Proof of corollary 3.1.8. - We will prove at the same time assertions (i) and (ii). We set $\widetilde{\alpha}=\alpha+2$ in case (i) and $\widetilde{\alpha}=\alpha$ in case (ii). We decompose

$$
u_{1} u_{2}=T_{u_{1}} u_{2}+T_{u_{2}} u_{1}+R\left(u_{1}, u_{2}\right) .
$$

Let $\gamma \in \mathbb{N}^{d},|\gamma| \leqslant 2 M-d-1$. If $|\gamma| \leqslant M-d-1$, we remark using notation (3.1.16) that $x^{\gamma} T_{u_{1}} u_{2}$ may be written as a linear combination of expressions of type $\widetilde{T}_{\left(x^{\gamma^{\prime}} u_{1}\right)} u_{2}$ with $\left|\gamma^{\prime}\right| \leqslant|\gamma|$. Actually, arguing by induction, only the case $|\gamma|=1$ has to be considered. One then writes

$$
x T_{u_{1}} u_{2}=\sum_{j \geqslant 1}\left(\left[x, S_{j-1}\right] u_{1}\right)\left(\Delta_{j} u_{2}\right)+T_{\left(x u_{1}\right)} u_{2}
$$

and remarks that $\left[x, S_{j-1}\right]=\chi_{j}(D)$ for a function $\chi_{j}(\xi)$ satisfying the second condition (3.1.15).

By assumption, we have

$$
x^{\gamma^{\prime}} x^{\gamma^{\prime \prime}} u_{1} \in \underline{\widetilde{E}}_{\widetilde{\alpha}, \alpha^{\prime}, \infty}^{s-\sigma_{1}, s^{\prime}-\sigma_{1}^{\prime}-\left|\gamma^{\prime}\right|-\left|\gamma^{\prime \prime}\right|}
$$

for $\left|\gamma^{\prime \prime}\right| \leqslant d+1$. By lemma 3.1.9, we deduce $x^{\gamma^{\prime}} u_{1} \in \widetilde{E}_{\widetilde{\alpha}, \alpha^{\prime}, 1}^{s-\sigma^{\prime}-\sigma_{1}^{\prime}-\left|\gamma^{\prime}\right|-d-\delta} \subset$ $\widetilde{H}_{\widetilde{\alpha}, \alpha^{\prime}, 1}^{s-\sigma_{1}, s^{\prime}-\sigma_{1}^{\prime}-\left|\gamma^{\prime}\right|-d-\delta}$ for any $\delta>0$. We apply proposition 3.1 .3 (ii) and get

$$
x^{\gamma} T_{u_{1}} u_{2} \in E_{\alpha+\left(\widetilde{\alpha}+2 \alpha^{\prime}\right)_{+}, \alpha^{\prime}, 1}^{s-\sigma_{2}-\left(d-s+\sigma_{1}\right)_{+}, \sigma_{2}^{\prime}-\left[-s^{\prime}+\sigma_{1}^{\prime}+|\gamma|+\delta+\frac{d}{2}\right]_{+}} .
$$

Since $s \geqslant d, \sigma_{1}^{\prime} \in[0,1], \sigma_{1} \geqslant 0$ and, in case (i), $\alpha+\left(\widetilde{\alpha}+2 \alpha^{\prime}\right)_{+}=\alpha+\left(\alpha+2 \alpha^{\prime}+2\right)_{+} \geqslant \beta$, we see that this last space is contained inside $E_{\beta, \alpha^{\prime}, 1}^{\widehat{s}, \widehat{s}^{\prime}(\gamma)}$ in case (i), and in $E_{\alpha, \alpha^{\prime}, 1}^{\widehat{s}, \widehat{s}^{\prime}(\gamma)}$ in case (ii). When $|\gamma| \geqslant M-d$, we decompose $\gamma=\gamma_{1}+\gamma_{2}$ with $\left|\gamma_{1}\right|=M-d-1,\left|\gamma_{2}\right| \leqslant M$ 
and write $x^{\gamma} T_{u_{1}} u_{2}$ as a linear combination of terms $\widetilde{T}_{\left(x^{\left.\gamma_{1}^{\prime} u_{1}\right)}\right.}\left(x^{\gamma_{2}^{\prime}} u_{2}\right)$ with $\left|\gamma_{1}^{\prime}\right| \leqslant\left|\gamma_{1}\right|$, $\left|\gamma_{2}^{\prime}\right| \leqslant\left|\gamma_{2}\right|$. We get that $x^{\gamma} T_{u_{1}} u_{2}$ is then inside

$$
E_{\alpha+\left(\widetilde{\alpha}+2 \alpha^{\prime}\right)_{+}, \alpha^{\prime}, 1}^{s-\sigma_{2}-\left(s^{\prime}-\sigma_{2}^{\prime}-\left|\gamma_{2}\right|-\left[-s^{\prime}+\sigma_{1}^{\prime}+\left|\gamma_{1}\right|+\delta+\frac{d}{2}\right]_{+}\right.} .
$$

Since

$$
\left|\gamma_{2}\right|+\left[-s^{\prime}+\sigma_{1}^{\prime}+\left|\gamma_{1}\right|+\delta+\frac{d}{2}\right]_{+} \leqslant\left[-s^{\prime}+\lambda+1+|\gamma|+\delta+\frac{d}{2}\right]_{+}
$$

as $-s^{\prime}+\lambda+1+\left|\gamma_{1}\right|+\frac{d}{2}>0$ by (3.1.50), we get that (3.1.59) is contained in $E_{\beta, \alpha^{\prime}, 1}^{\widehat{s}, \widehat{s}^{\prime}(\gamma)}$ in case (i) and in $E_{\alpha, \alpha^{\prime}, 1}^{\widehat{s}, \hat{s}^{\prime}(\gamma)}$ in case (ii).

The proof that $x^{\gamma} T_{u_{2}} u_{1}$ belongs to $E_{\beta, \alpha^{\prime}, 1}^{\widehat{s}, \widehat{s}^{\prime}(\gamma)}$ (resp. $E_{\alpha, \alpha^{\prime}, 1}^{\widehat{s}, \widehat{s}^{\prime}(\gamma)}$ in case (i) (resp. in case (ii)) is similar: one has just to replace in (3.1.58), (3.1.59) the first lower index by $\widetilde{\alpha}+\left(\alpha+2 \alpha^{\prime}\right)_{+}$, which is larger than $\beta$ (resp. $\alpha$ ) in case (i) (resp. case (ii)).

Let us study $R\left(u_{1}, u_{2}\right)$. For $|\gamma| \leqslant 2 M-d-1$, we write $x^{\gamma} R\left(u_{1}, u_{2}\right)$ as a linear combination of terms of type $\widetilde{R}\left(x^{\gamma_{1}} u_{1}, x^{\gamma_{2}} u_{2}\right)$ with $\left|\gamma_{1}\right|+\left|\gamma_{2}\right| \leqslant|\gamma|,\left|\gamma_{1}\right| \leqslant M-d-1$, $\left|\gamma_{2}\right| \leqslant M$. We will thus have $x^{\gamma_{2}} u_{2} \in \underline{\widetilde{E}}_{\alpha, \alpha^{\prime}, \infty}^{s-\sigma^{\prime}-\sigma_{2}^{\prime}-\left|\gamma_{2}\right|}$ and by lemma 3.1.9 $x^{\gamma_{1}} u_{1} \in$ $\widetilde{E}_{\widetilde{\alpha}, \alpha^{\prime}, 1}^{s-s^{\prime}-\sigma_{1}^{\prime}-\left|\gamma_{1}\right|-d-\delta} \subset \widetilde{H}_{\widetilde{\alpha}, \alpha^{\prime}, 1}^{s-\sigma_{1}, s^{\prime}-\sigma_{1}^{\prime}-\left|\gamma_{1}\right|-d-\delta}$. In case (i), we apply (i) of proposition 3.1.5. We check that for $\delta>0$ small enough,

$$
\begin{aligned}
2 s+2 s^{\prime}-\left(\sigma_{1}+\sigma_{2}\right)-\left(\sigma_{1}^{\prime}+\sigma_{2}^{\prime}\right)-d-\delta-|\gamma| & >0 \\
2 s-\left(\sigma_{1}+\sigma_{2}\right) & >2 \alpha^{\prime} \\
2\left(\alpha+\alpha^{\prime}+1\right) & <0
\end{aligned}
$$

because of assumptions (3.1.50), (3.1.51), (3.1.52), i.e. all conditions (3.1.23) are satisfied. Consequently $\widetilde{R}\left(x^{\gamma_{1}} u_{1}, x^{\gamma_{2}} u_{2}\right)$ belongs to

$$
E_{2 \alpha+2+d, 2 \alpha^{\prime}-d / 2,1}^{2 s-\left(\sigma_{1}+\sigma_{2}\right)-d, 2 s^{\prime}-\left(\sigma_{1}^{\prime}+\sigma_{2}^{\prime}\right)-|\gamma|-\delta-\frac{d}{2}}
$$

which is contained, since $s \geqslant d$ and $\alpha^{\prime}>d / 2$, inside $E_{\beta, \alpha^{\prime}, 1}^{\widehat{s}, \widehat{s}^{\prime}}$. This concludes the proof of the fact that $x^{\gamma} u_{1} u_{2} \in \widetilde{E}_{\beta, \alpha^{\prime}, 1}^{\widehat{s}, \widehat{S}^{\prime}(\gamma)}$ in case (i) since (3.1.51) implies $\frac{\beta}{2}+\alpha^{\prime}>0$ whence $\widetilde{E}_{\beta, \alpha^{\prime}, 1}^{\widehat{s}, \widehat{s}^{\prime}(\gamma)}=E_{\beta, \alpha^{\prime}, 1}^{\widehat{s}, \widehat{s}^{\prime}(\gamma)}$ by lemma 3.1.2.

In case (ii), one has to check (3.1.25) to apply proposition 3.1.5 i.e. to verify conditions (3.1.60) with the last inequality replaced by $\alpha+\alpha^{\prime}<0, \alpha^{\prime}>d / 2$. This follows again from (3.1.50), (3.1.52), (3.1.54), and implies that $\widetilde{R}\left(x^{\gamma_{1}} u_{1}, x^{\gamma_{2}} u_{2}\right) \in E_{\alpha, \alpha^{\prime}, 1}^{\widehat{s}, \widehat{s}^{\prime}(\gamma)}$.

To conclude the proof of the corollary, we still have to get the $L^{\infty}$ estimate involved

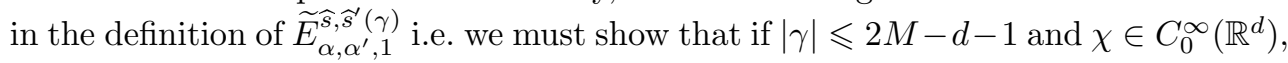
Supp $\chi \subset B(0,1)$,

$$
\left(\left\|\phi_{q}(x) \chi(D / \sqrt{t})\left(x^{\gamma} u_{1} u_{2}\right)\right\|_{L^{\infty}(d t d x)}\right)_{q} \in \ell_{q}^{1} .
$$


Consider $A(t, D)=\sum_{k ; 2^{k}>10 \sqrt{t}} \Delta_{k}$ and split $\gamma=\gamma_{1}+\gamma_{2}$, with the inequalities $\left|\gamma_{1}\right| \leqslant M-d-1,\left|\gamma_{2}\right| \leqslant M$. We have

$$
\begin{aligned}
\phi_{q}(x) \chi(D / \sqrt{t})\left(\left(x^{\gamma_{1}} u_{1}\right)\right. & \left.A(t, D)\left(x^{\gamma_{2}} u_{2}\right)\right) \\
= & \left.\sum_{\substack{k, k^{\prime} \\
\left|k-k^{\prime}\right| \leqslant N_{0} \\
2^{k}>10 \sqrt{t}}} \phi_{q}(x) \chi(D / \sqrt{t})\left[\Delta_{k^{\prime}}\left(x^{\gamma_{1}} u_{1}\right) \Delta_{k}\left(x^{\gamma_{2}} u_{2}\right)\right)\right] .
\end{aligned}
$$

for a large enough integer $N_{0}$. The general term in the sum (3.1.62) may be written

$$
\sum_{q^{\prime}} \phi_{q}(x) \chi(D / \sqrt{t}) \widetilde{\phi}_{q^{\prime}}\left[\phi_{q^{\prime}} \Delta_{k^{\prime}}\left(x^{\gamma_{1}} u_{1}\right) \Delta_{k}\left(x^{\gamma_{2}} u_{2}\right)\right] .
$$

For fixed $t$, the $L^{\infty}(d x)$ norm of (3.1.63) is smaller than

$$
C_{N} \sum_{q^{\prime}}\left\langle q-q^{\prime}\right\rangle^{-N} t^{d / 2}\left\|\phi_{q^{\prime}} \Delta_{k^{\prime}}\left(x^{\gamma_{1}} u_{1}\right)(t, \cdot)\right\|_{L^{2}}\left\|\Delta_{k}\left(x^{\gamma_{2}} u_{2}\right)(t, \cdot)\right\|_{L^{2}} .
$$

The $L^{\infty}$ norm of (3.1.62) is thus smaller than the $q^{\prime}$ sum of $C_{N}\left\langle q-q^{\prime}\right\rangle^{-N}$ times

$$
\begin{aligned}
& \sum_{k ; \sqrt{t}<2^{k}<t} t^{d / 2}\left(\frac{2^{k}}{\sqrt{t}}\right)^{-2 s+\left(\sigma_{1}+\sigma_{2}\right)}\left(\frac{2^{k}}{t}\right)^{2 \alpha^{\prime}} c_{k q^{\prime}} \\
&+\sum_{k ; t<2^{k}} t^{d / 2}\left(\frac{2^{k}}{\sqrt{t}}\right)^{-2 s+\left(\sigma_{1}+\sigma_{2}\right)}\left(\frac{2^{k}}{t}\right)^{-2 s^{\prime}+\left(\sigma_{1}^{\prime}+\sigma_{2}^{\prime}\right)+|\gamma|+d+\delta} c_{k q^{\prime}} \\
& \leqslant \sum_{k ; \sqrt{t}<2^{k}<t} t^{d / 2-\alpha^{\prime}}\left(\frac{2^{k}}{\sqrt{t}}\right)^{-2 s+\left(\sigma_{1}+\sigma_{2}\right)+2 \alpha^{\prime}} c_{k q^{\prime}} \\
& \quad+\sum_{k ; t<2^{k}} t^{\frac{d}{2}+\frac{\sigma_{1}+\sigma_{2}}{2}-s}\left(\frac{2^{k}}{t}\right)^{-2\left(s+s^{\prime}\right)+\left(\sigma_{1}+\sigma_{2}+\sigma_{1}^{\prime}+\sigma_{2}^{\prime}\right)+|\gamma|+d+\delta} c_{k q^{\prime}}
\end{aligned}
$$

for a positive $\delta$ and a $\ell_{k}^{1} \ell_{q^{\prime}}^{1}$ sequence $\left(c_{k q^{\prime}}\right)_{k q^{\prime}}$. Since, by (3.1.50), $\alpha^{\prime}>d / 2, s>$ $\alpha^{\prime}+\frac{\sigma_{1}+\sigma_{2}}{2}>\frac{d}{2}+\frac{\sigma_{1}+\sigma_{2}}{2}$, and because of (3.1.52), we see that the last expression is smaller than $C \sum_{k} 2^{-k \delta^{\prime}} c_{k q^{\prime}}$, for some positive $\delta^{\prime}$, i.e. the $L^{\infty}$ norm of (3.1.62) is smaller than a $\ell_{q}^{1}$ sequence. We are thus reduced to the study of

$$
\left\|\phi_{q}\left[\chi(D / \sqrt{t})\left(\left(x^{\gamma_{1}} u_{1}\right)(\operatorname{Id}-A(t, D))\left(x^{\gamma_{2}} u_{2}\right)\right)\right]\right\|_{L^{\infty}} .
$$

Repeating the preceding reasoning, we further reduce ourselves to

$$
\left\|\phi_{q}\left[\chi(D / \sqrt{t})\left((\operatorname{Id}-A(t, D))\left(x^{\gamma_{1}} u_{1}\right)(\operatorname{Id}-A(t, D))\left(x^{\gamma_{2}} u_{2}\right)\right)\right]\right\|_{L^{\infty}} .
$$

We get that this sequence is in $\ell_{q}^{1}$ applying lemma 3.1.2 with $\psi(t, \xi)=1-A(t, \xi)$. This concludes the proof. 


\subsection{Conjugation by an oscillatory exponential}

Our aim in this section is to study expressions of type

$$
e^{-i m \theta} T_{w}\left((1-\chi)(D / \sqrt{t}) e^{i m \theta}\right)
$$

where $m \in \mathbb{Z}^{*}$ and $w \in \widetilde{E}_{\alpha, \alpha^{\prime}, r}^{s, s^{\prime}}$. We want to show that (3.2.1) has essentially the same smoothness as $w$. This will follow from the fact that making act a $D_{x} / \sqrt{t}$ or a $D_{x} / t$ derivative on (3.2.1) gives either a term like (3.2.1) - with $w$ replaced by $\frac{D_{x}}{\sqrt{t}} w$ or $\frac{D_{x}}{t} w$ - or two contributions coming from the action of the derivative on the exponentials, that will compensate each other modulo remainders.

Let us introduce some notations:

Definition 3.2.1. - For $\mu \in \mathbb{R}, \nu \in \mathbb{R}_{+}$one denotes by $\Sigma_{\nu}^{\mu}$ the space of smooth functions $(t, \xi) \mapsto a(t, \xi)$ defined on $\left[1,+\infty\left[\times \mathbb{R}^{d}\right.\right.$ with values in $\mathbb{C}$, satisfying the following inequalities

$$
\left|\left(t \partial_{t}\right)^{k}\left(\sqrt{t} \partial_{\xi}\right)^{\gamma} a(t, \xi)\right| \leqslant C_{k \gamma}\left(\frac{\langle\xi\rangle}{t}\left(1+\frac{\langle\xi\rangle}{t}\right)^{-1}\right)^{\nu}\left(1+\frac{\langle\xi\rangle}{\sqrt{t}}\right)^{\mu-|\gamma|},
$$

for any $\gamma$ when $|\xi|>c \sqrt{t}$, and for $\gamma=0$ when $|\xi| \leqslant c \sqrt{t}$.

We shall also denote by $S^{\mu}$ the space of usual symbols of order $\mu$ i.e. smooth functions $b$ satisfying for any $\gamma \in \mathbb{N}^{d}$

$$
\left|\partial_{x}^{\gamma} b(x)\right| \leqslant C_{\gamma}\langle x\rangle^{-|\gamma|+\mu} .
$$

Proposition 3.2.2. - Let $s, s^{\prime}, \alpha, \alpha^{\prime}, r, \mu, \rho, \nu$ be real numbers satisfying

$$
\begin{gathered}
r \in[1,+\infty], \quad \alpha+\alpha^{\prime}<d / 2, \quad \alpha^{\prime}>d / 2 \\
\rho>\mu \geqslant 0, \quad \nu \geqslant 0 .
\end{gathered}
$$

Let $a(t, \xi) \in \Sigma_{\nu}^{-\rho}$ and $b(x) \in S^{\mu}$. Assume moreover that for some $c>0$,

$$
\operatorname{Supp} a \subset\{(t, \xi) ;|\xi|>c \sqrt{t}\} \text {. }
$$

and that

$$
\left(\frac{\alpha}{2}+\alpha^{\prime}>0, \alpha+\alpha^{\prime}+\frac{d}{2}>0\right) \text { or }\left(\frac{\alpha}{2}+\alpha^{\prime}<0, \alpha+\alpha^{\prime}+\frac{d}{2}+\nu>0\right) .
$$

Then if $w \in \widetilde{E}_{\alpha, \alpha^{\prime}, r}^{s, s^{\prime}}$, we have for any $m \in \mathbb{Z}^{*}$

$$
e^{-i m \theta} b(\sqrt{t} x) T_{w}\left(a(t, D) e^{i m \theta}\right) \in \widetilde{E}_{\alpha+\zeta, \alpha^{\prime}, r}^{s+\rho-\mu, s^{\prime}} \subset \widetilde{E}_{\alpha, \alpha^{\prime}, r}^{s+\rho-\mu, s^{\prime}}
$$

where $\zeta$ is any number in $] 0, \min \left(\rho-\mu, d, \frac{d}{2}-\alpha-\alpha^{\prime}\right)[$.

We write by definition of paraproduct

$$
\begin{array}{r}
e^{-i m \theta} b(\sqrt{t} x) T_{w}\left[a(t, D) e^{i m \theta}\right]=\sum_{k}\left(S_{k-1} w\right) g_{k} \\
g_{k}=e^{-i m \theta} b(\sqrt{t} x) a(t, D) \Delta_{k}\left(e^{i m \theta}\right) .
\end{array}
$$


The assumption (3.2.5) implies that in the right hand side of (3.2.8) the summation is taken for $k$ satisfying $2^{k}>c \sqrt{t}$. We begin by the following lemma:

Lemma 3.2.3. - For any $N \in \mathbb{N}$, one has the following estimates, uniformly in $t \geqslant 1, k$ with $2^{k}>\sqrt{t}$ :

$$
\begin{aligned}
& \left\|\Delta_{j} g_{k}(t, \cdot)\right\|_{L^{2}} \\
\leqslant & C_{N} 2^{-j \frac{d}{2}}\left(\frac{2^{j+k}}{t}\right)^{d}\left(1+\frac{2^{j+k}}{t}\right)^{-N}\left(\frac{2^{k}}{\sqrt{t}}\right)^{-\rho+\mu}\left[\frac{2^{k}}{t}\left(1+\frac{2^{k}}{t}\right)^{-1}\right]^{\nu}
\end{aligned}
$$

$(3.2 .11) \quad\left\|S_{j} g_{k}(t, \cdot)\right\|_{L^{\infty}}$

$$
\leqslant C_{N}\left(\frac{2^{j+k}}{t}\right)^{d}\left(1+\frac{2^{j+k}}{t}\right)^{-d}\left(\frac{2^{k}}{\sqrt{t}}\right)^{-\rho+\mu}\left[\frac{2^{k}}{t}\left(1+\frac{2^{k}}{t}\right)^{-1}\right]^{\nu} .
$$

Proof. - Compute the Fourier transform of $b(\sqrt{t} x) e^{-i m \theta}$ as the oscillatory integral

$$
\int e^{-i x \xi-i m t x^{2} / 4} b(\sqrt{t} x) d x=\frac{1}{t^{d / 2}} e^{i \xi^{2} / m t} B(\xi / \sqrt{t})
$$

where

$$
B(\eta)=\int e^{-i m x^{2} / 4} b(x-2 \eta / m) d x .
$$

If $L=\left(1+m^{2} x^{2}\right)^{-1}\left(1-2 m x \cdot D_{x}\right)$, so that $L\left(e^{-i m x^{2} / 4}\right)=e^{-i m x^{2} / 4}$, we have

$$
\left|\left({ }^{t} L\right)^{k}[b(x-2 \eta / m)]\right| \leqslant C_{k}\langle x\rangle^{-k}(1+|x-2 \eta / m|)^{\mu}
$$

whence, integrating by parts in (3.2.13), the estimate $B(\eta) \leqslant C\langle\eta\rangle^{\mu}$. Treating the derivatives in the same way, we get that $B \in S^{\mu}$. We now write the Fourier transform of $g_{k}$ as a multiple of

$$
\mathcal{F}\left(e^{-i m \theta} b(\sqrt{t} x)\right) *\left[a(t, \eta) \varphi\left(2^{-k} \eta\right) \frac{e^{-i \eta^{2} / m t}}{t^{d / 2}}\right]
$$

that is, using (3.2.12), (3.2.13),

$$
e^{i \xi^{2} / m t}\left(\frac{2^{k}}{t}\right)^{d} \int e^{-2 i \frac{\xi \eta}{m t} 2^{k}} B\left(\frac{\xi-2^{k} \eta}{\sqrt{t}}\right) \varphi(\eta) a\left(t, 2^{k} \eta\right) d \eta .
$$

Integrating by parts in $\eta$ we obtain an estimate of the modulus of (3.2.14) by

$$
\begin{aligned}
C\left(\frac{2^{k}}{t}\right)^{d}( & \left.+\frac{2^{k}|\xi|}{t}\right)^{-N}\left[\frac{2^{k}}{t}\left(1+\frac{2^{k}}{t}\right)^{-1}\right]^{\nu}\left(\frac{2^{k}}{\sqrt{t}}\right)^{-\rho} \\
& \times \sum_{N^{\prime}=0}^{N} \int\left(1+\frac{\left|\xi-2^{k} \eta\right|}{\sqrt{t}}\right)^{\mu-N^{\prime}}\left(\frac{2^{k}}{\sqrt{t}}\right)^{N^{\prime}} \varphi_{1}(\eta) d \eta,
\end{aligned}
$$

where we used (3.2.2), $B \in S^{\mu}, 2^{k}>c \sqrt{t}$, and where $\varphi_{1}$ has the same support as $\varphi$. Consider first $N^{\prime}$ with $\mu-N^{\prime} \leqslant 0$. When the integration is made over a domain 
$\left|\xi-2^{k} \eta\right|>c 2^{k}$ for a small $c>0$, we get an upper bound

$$
C\left(\frac{2^{k}}{t}\right)^{d}\left(1+\frac{2^{k}|\xi|}{t}\right)^{-N}\left[\frac{2^{k}}{t}\left(1+\frac{2^{k}}{t}\right)^{-1}\right]^{\nu}\left(\frac{2^{k}}{\sqrt{t}}\right)^{\mu-\rho} .
$$

On the other hand, the contribution of the integration for $\left|\xi-2^{k} \eta\right|<c 2^{k}$ gives, since this implies $|\xi| \sim 2^{k}$, and since $-2 N+N^{\prime} \leqslant-N$,

$$
C\left(\frac{2^{k}}{t}\right)^{d}\left[\frac{2^{k}}{t}\left(1+\frac{2^{k}}{t}\right)^{-1}\right]^{\nu}\left(\frac{2^{k}}{\sqrt{t}}\right)^{-\rho-N}
$$

which is still of form (3.2.15) changing the value of $N$.

On the other hand, for $N^{\prime}$ such that $\mu-N^{\prime}>0$, we get immediately an upper bound of type (3.2.15), changing the value of $N$.

Inequality (3.2.10) (resp. (3.2.11)) follows from estimate (3.2.15) computing the $L^{2}$ (resp. $\left.L^{1}\right)$ norm of $\widehat{\Delta_{j} g_{k}}(\xi)$ over a ring (resp. a ball) of radius $2^{j}$.

Proof of proposition 3.2.2. - We decompose (3.2.8) as

$$
\sum_{k ; 2^{k}>c \sqrt{t}} T_{g_{k}}\left(S_{k-1} w\right)+\sum_{k ; 2^{k}>c \sqrt{t}} R\left(g_{k}, S_{k-1} w\right)+\sum_{k ; 2^{k}>c \sqrt{t}} T_{S_{k-1} w} g_{k}
$$

and we shall estimate successively for a fixed $j$

$$
\begin{gathered}
\sum_{k ; 2^{k}>c \sqrt{t}} \Delta_{j}\left(T_{g_{k}}\left(S_{k-1} w\right)\right) \\
\sum_{k ; 2^{k}>c \sqrt{t}} \Delta_{j}\left(R\left(g_{k}, S_{k-1} w\right)\right) \\
\sum_{k ; 2^{k}>c \sqrt{t}} \Delta_{j}\left(T_{S_{k-1} w} g_{k}\right) .
\end{gathered}
$$

The assumptions on $a, b$ of proposition 3.2.2 will enter the proof only through the estimates (3.2.10), (3.2.11). Since the parameters $\rho$ and $\mu$ appear in the right hand sides of these inequalities only in the difference $\rho-\mu$, we can without reducing the generality assume $\mu=0, \rho>0$.

Estimate of (3.2.16). - We write the product of (3.2.16) with a function $\phi_{q}$ as

$$
\sum_{\substack{j^{\prime} \\\left|j-j^{\prime}\right| \leqslant N_{0}}} \sum_{\substack{k \\ 2^{k}>c \sqrt{t} \\ k \geqslant j-N_{0}}} \sum_{q^{\prime}}\left(\phi_{q} \Delta_{j} \widetilde{\phi}_{q^{\prime}}\right)\left(\left(S_{j^{\prime}-1} g_{k}\right) \phi_{q^{\prime}} \Delta_{j^{\prime}} S_{k-1} w\right)
$$

for a large enough integer $N_{0}$. We multiply by $\Gamma_{\ell}(t)$ and compute the $L^{p}\left(\frac{d t}{t}, L^{2}(d x)\right)$ norm for $p=2$ or $p=\infty$ of (3.2.19). We get an upper bound

$$
\begin{aligned}
\sum_{\substack{j^{\prime} \\
\left|j-j^{\prime}\right| \leqslant N_{0}}} \sum_{\substack{k \\
2^{k}>c 2^{\ell / 2} \\
k \geqslant j-N_{0}}} \sum_{q^{\prime}} C_{N}\left\langle q-q^{\prime}\right\rangle^{-N}\left\|\Gamma_{\ell}(t)\left(S_{j^{\prime}-1} g_{k}\right)\right\|_{L^{\infty}(d t d x)} \\
\times\left\|\Gamma_{\ell}(t) \phi_{q^{\prime}} \Delta_{j^{\prime}} S_{k-1} w\right\|_{L^{p}\left(\frac{d t}{t}, L^{2}(d x)\right)} .
\end{aligned}
$$


The last norm in the above formula is bounded from above by

$$
C 2^{(j-\ell / 2) \alpha+(j-\ell) \alpha^{\prime}}\left(1+2^{j-\ell / 2}\right)^{-s-\alpha}\left(1+2^{j-\ell}\right)^{-\widetilde{s}^{\prime}-\alpha^{\prime}} c_{j q^{\prime}}
$$

with $\widetilde{s}^{\prime}=s^{\prime}$ if $p=\infty$ and $\widetilde{s}^{\prime}=s^{\prime}+1 / 2$ if $p=2,\left(c_{j q^{\prime}}\right)_{j q^{\prime}}$ in the unit ball of $\ell_{j}^{2} \ell_{q^{\prime}}^{r}$. On the other hand, we have by $(3.2 .11)$

$$
\begin{aligned}
& \sum_{\substack{k \\
k \geqslant \frac{\ell}{2}-N_{0} \\
k \geqslant j-N_{0}}}\left\|\Gamma_{\ell}(t) S_{j^{\prime}-1} g_{k}\right\|_{L^{\infty}(d t d x)} \\
& \quad \leqslant \sum_{\substack{k \\
k \geqslant \frac{\ell}{2}-N_{0} \\
k \geqslant j-N_{0}}} 2^{(j+k-\ell) d}\left(1+2^{j+k-\ell}\right)^{-d} 2^{-(k-\ell / 2) \rho}\left(2^{k-\ell}\left(1+2^{k-\ell}\right)^{-1}\right)^{\nu} .
\end{aligned}
$$

Denote by $\zeta^{\prime}$ any positive number with $\zeta^{\prime}<\min [\rho, d]$. When $\ell / 2 \geqslant j$, this last sum is smaller than

$$
\sum_{k>\ell-j} 2^{-(k-\ell / 2) \rho}+\sum_{k ; \ell-j \geqslant k \geqslant \ell / 2-N_{0}} 2^{(j+k-\ell) d-(k-\ell / 2) \rho} \leqslant C 2^{(j-\ell / 2) \zeta^{\prime}} .
$$

When $j>\ell / 2$, we have the estimate

$$
\sum_{k \geqslant j-N_{0}} 2^{-(k-\ell / 2) \rho} \leqslant C 2^{-(j-\ell / 2) \rho} .
$$

We see that (3.2.22) is smaller than

$$
2^{(j-\ell / 2) \zeta^{\prime}}\left(1+2^{j-\ell / 2}\right)^{-\rho-\zeta^{\prime}} .
$$

Consequently, (3.2.20), (3.2.21), (3.2.22), (3.2.23) show that (3.2.16) belongs to $E_{\alpha+\zeta^{\prime}, \alpha^{\prime}, r}^{s+\rho, s^{\prime}}$

Estimate of (3.2.17). — We write the product of (3.2.17) with a function $\phi_{q}$ as

$$
\sum_{\substack{j^{\prime}, j^{\prime \prime} \\ j^{\prime}-j^{\prime \prime} \mid \leqslant N_{0} \\ j^{\prime} \geqslant j-N_{0}}} \sum_{\substack{k \\ 2^{k}>c \sqrt{t} \\ k \geqslant j^{\prime \prime}-N_{0}}} \sum_{q^{\prime}}\left(\phi_{q} \Delta_{j} \widetilde{\phi}_{q^{\prime}}\right)\left(\left(\Delta_{j^{\prime}} g_{k}\right)\left(\phi_{q^{\prime}} \Delta_{j^{\prime \prime}} S_{k-1} w\right)\right)
$$

for a large enough integer $N_{0}$. We multiply by $\Gamma_{\ell}(t)$ and compute the $L^{p}\left(\frac{d t}{t}, L^{2}(d x)\right)$ norm for $p=2$ or $p=\infty$ of (3.2.24). We get an upper bound

$$
\begin{aligned}
\sum_{\substack{j^{\prime}, j^{\prime \prime} \\
j^{\prime}-j^{\prime \prime} \mid \leqslant N_{0} \\
j^{\prime} \geqslant j-N_{0}}} & \sum_{\substack{k \\
k \geqslant \ell / 2-N_{0} \\
k \geqslant j^{\prime \prime}-N_{0}}} \sum_{q^{\prime}} C_{N}\left\langle q-q^{\prime}\right\rangle^{-N} \\
& \times 2^{j d / 2}\left\|\Delta_{j^{\prime}} g_{k}\right\|_{L^{\infty}\left(d t, L^{2}(d x)\right)}\left\|\Gamma_{\ell}(t) \phi_{q^{\prime}} \Delta_{j^{\prime \prime}} S_{k-1} w\right\|_{L^{p}\left(\frac{d t}{t}, L^{2}(d x)\right)} .
\end{aligned}
$$

for a large enough $N_{0}$. We use (3.2.10) to estimate the first norm in (3.2.25) and remind that the second norm is bounded from above by (3.2.21) with $j$ replaced by 
$j^{\prime \prime} \sim j^{\prime}$. Consequently, (3.2.25) is smaller than

$$
\begin{gathered}
\sum_{\substack{j^{\prime} \\
j^{\prime} \geqslant j-N_{0}}} \sum_{\substack{k \\
k \geqslant \ell / 2-N_{0} \\
k \geqslant j^{\prime}-N_{0}}} \sum_{q^{\prime}} C_{N}\left\langle q-q^{\prime}\right\rangle^{-N} 2^{\left(j^{\prime}+k-\ell\right) d+\left(j-j^{\prime}\right) d / 2}\left(1+2^{j^{\prime}+k-\ell}\right)^{-N} \\
\times 2^{-(k-\ell / 2) \rho}\left[2^{k-\ell}\left(1+2^{k-\ell}\right)^{-1}\right]^{\nu} 2^{\left(j^{\prime}-\ell / 2\right) \alpha} 2^{\left(j^{\prime}-\ell\right) \alpha^{\prime}} c_{j^{\prime} q^{\prime}}
\end{gathered}
$$

since the terms in $s, \widetilde{s}^{\prime}$ of $(3.2 .21)$ can always be compensated for by $\left(1+2^{j^{\prime}+k-\ell}\right)^{-N}$ as $k \geqslant \frac{\ell}{2}-N_{0}$. This expression is smaller than

$$
\begin{array}{r}
\sum_{\substack{j^{\prime} \\
j^{\prime} \geqslant j-N_{0}}} \sum_{q^{\prime}} C_{N}\left\langle q-q^{\prime}\right\rangle^{-N} c_{j^{\prime} q^{\prime}} 2^{\left(j-j^{\prime}\right) d / 2+\left(j^{\prime}-\ell / 2\right) \alpha+\left(j^{\prime}-\ell\right) \alpha^{\prime}}\left(1+2^{j^{\prime}-\ell / 2}\right)^{-N+d} \\
\times \sum_{\substack{k \\
k \geqslant \ell / 2-N_{0} \\
k \geqslant j^{\prime}-N_{0}}} 2^{\left(j^{\prime}+k-\ell\right) d}\left(1+2^{j^{\prime}+k-\ell}\right)^{-d} 2^{-(k-\ell / 2) \rho}\left[2^{k-\ell}\left(1+2^{k-\ell}\right)^{-1}\right]^{\nu} \\
\leqslant \sum_{\substack{j^{\prime} \\
j^{\prime} \geqslant j-N_{0}}} \sum_{q^{\prime}} C_{N}\left\langle q-q^{\prime}\right\rangle^{-N} c_{j^{\prime} q^{\prime}} 2^{\left(j-j^{\prime}\right) d / 2+\left(j^{\prime}-\ell / 2\right) \alpha+\left(j^{\prime}-\ell\right) \alpha^{\prime}} \\
\times 2^{\left(j^{\prime}-\ell / 2\right) \zeta^{\prime}}\left(1+2^{j^{\prime}-\ell / 2}\right)^{-\rho-\zeta^{\prime}-N+d}
\end{array}
$$

by the estimate (3.2.23) of (3.2.22). If we take $\zeta<\min \left[\zeta^{\prime}, \frac{d}{2}-\alpha-\alpha^{\prime}\right]$, we obtain an upper bound of type

$$
C c_{j q}^{\prime} 2^{(j-\ell / 2)(\alpha+\zeta)+(j-\ell) \alpha^{\prime}}\left(1+2^{j-\ell / 2}\right)^{-N^{\prime}}
$$

for any $N^{\prime} \in \mathbb{N}$, with $\left(c_{j q}^{\prime}\right)_{j q}$ in $\ell_{j}^{2} \ell_{q}^{r}$. This shows that (3.2.17) is in $E_{\alpha+\zeta, \alpha^{\prime}, r}^{+\infty,+\infty}$.

Estimate of (3.2.18). - We write the product of (3.2.18) with a function $\phi_{q}$ as

$$
\sum_{\substack{j^{\prime} \\\left|j-j^{\prime}\right| \leqslant N_{0}}} \sum_{\substack{k \\ 2^{k}>c \sqrt{t}}} \sum_{q^{\prime}}\left(\phi_{q} \Delta_{j} \widetilde{\phi}_{q^{\prime}}\right)\left(\left(\phi_{q^{\prime}} S_{j^{\prime}-1} S_{k-1} w\right)\left(\Delta_{j^{\prime}} g_{k}\right)\right)
$$

for a large enough integer $N_{0}$. We multiply by $\Gamma_{\ell}(t)$ and compute the $L^{p}\left(\frac{d t}{t}, L^{2}(d x)\right)$ norm for $p=2$ or $p=\infty$. We get the upper bound

$$
\begin{array}{r}
\sum_{\substack{j^{\prime} \\
\left|j-j^{\prime}\right| \leqslant N_{0}}} \sum_{\substack{k \\
k \geqslant \ell / 2-N_{0}}} \sum_{q^{\prime}} C_{N}\left\langle q-q^{\prime}\right\rangle^{-N}\left\|\phi_{q^{\prime}} S_{j^{\prime}-1} S_{k-1} w \Gamma_{\ell}(t)\right\|_{L^{p}\left(\frac{d t}{t}, L^{\infty}(d x)\right)} \\
\times\left\|\Delta_{j^{\prime}} g_{k} \Gamma_{\ell}(t)\right\|_{L^{\infty}\left(d t, L^{2}(d x)\right)} .
\end{array}
$$

We consider first the case $\alpha / 2+\alpha^{\prime}>0, \alpha+\alpha^{\prime}+d / 2>0$. Let us show that for some $\mu_{0} \in \mathbb{N}$ and some sequence $\left(c_{j q^{\prime}}\right)_{j q^{\prime}}$ in $\ell_{j}^{2} \ell_{q^{\prime}}^{r}$ we have the estimate

$$
\begin{aligned}
\left\|\phi_{q^{\prime}} S_{j^{\prime}-1} S_{k-1} w \Gamma_{\ell}(t)\right\|_{L^{p}\left(\frac{d t}{t}, L^{\infty}(d x)\right)} & \\
& \leqslant C c_{j q^{\prime}} 2^{j^{\prime} d / 2+\left(j^{\prime}-\ell / 2\right) \alpha+\left(j^{\prime}-\ell\right) \alpha^{\prime}}\left(1+2^{j^{\prime}-\ell / 2}\right)^{\mu_{0}} .
\end{aligned}
$$


We write

$$
\begin{aligned}
& \left\|\phi_{q^{\prime}} S_{j^{\prime}-1} S_{k-1} w \Gamma_{\ell}(t)\right\|_{L^{p}\left(\frac{d t}{t}, L^{\infty}(d x)\right)} \\
& \quad \leqslant C_{N} \sum_{q^{\prime \prime}} \sum_{k^{\prime} \leqslant j^{\prime}-2}\left\langle q^{\prime}-q^{\prime \prime}\right\rangle^{-N} 2^{k^{\prime} d / 2}\left\|\phi_{q^{\prime \prime}} \Delta_{k^{\prime}} w \Gamma_{\ell}(t)\right\|_{L^{p}\left(\frac{d t}{t}, L^{2}(d x)\right)} .
\end{aligned}
$$

When $j^{\prime}<\ell / 2$ we get an estimate of type

$$
\sum_{k^{\prime} \leqslant j^{\prime}-2} 2^{k^{\prime} d / 2+\left(k^{\prime}-\ell / 2\right) \alpha+\left(k^{\prime}-\ell\right) \alpha^{\prime}} c_{k^{\prime} q^{\prime}}^{\prime}
$$

for some $\ell_{k^{\prime}}^{2} \ell_{q^{\prime}}^{r}$ sequence $\left(c_{k^{\prime} q^{\prime}}^{\prime}\right)_{k^{\prime} q^{\prime}}$. Since $\frac{d}{2}+\alpha+\alpha^{\prime}>0$, (3.2.29) follows. When $j^{\prime}>\ell / 2$, we estimate the left hand side of (3.2.29) by

$\sum_{k^{\prime}<\ell / 2} 2^{k^{\prime} d / 2+\left(k^{\prime}-\ell / 2\right) \alpha+\left(k^{\prime}-\ell\right) \alpha^{\prime}} c_{k^{\prime} q^{\prime}}^{\prime}+\sum_{k^{\prime} ; \ell / 2 \leqslant k^{\prime}} 2^{k^{\prime} d / 2+\left(k^{\prime}-\ell\right) \alpha^{\prime}-\left(k^{\prime}-\ell / 2\right) \mu_{1}} c_{k^{\prime} q^{\prime}}^{\prime} 2^{\left(j^{\prime}-\ell / 2\right) \mu_{2}}$

for large enough $\mu_{1}$ and $\mu_{2}$. This is smaller than

$$
2^{\frac{\ell}{2} \frac{d}{2}-\frac{\ell}{2} \alpha^{\prime}+\left(j^{\prime}-\frac{\ell}{2}\right) \mu_{2}} c_{\ell q^{\prime}}^{\prime \prime}=2^{j^{\prime} \frac{d}{2}+\left(j^{\prime}-\ell\right) \alpha^{\prime}} c_{\ell q^{\prime}}^{\prime \prime}{ }^{\left(j^{\prime}-\frac{\ell}{2}\right)\left(\mu_{2}-\alpha^{\prime}-\frac{d}{2}\right)}
$$

for some $\ell_{\ell}^{2} \ell_{q^{\prime}}^{r}$ sequence $\left(c_{\ell q^{\prime}}^{\prime \prime}\right)_{\ell q^{\prime}}$, whence (3.2.29).

Next, we compute from (3.2.10)

$$
\begin{aligned}
& \sum_{k \geqslant \ell / 2-N_{0}}\left\|\Delta_{j^{\prime}} g_{k} \Gamma_{\ell}(t)\right\|_{L^{\infty}\left(d t, L^{2}\right)} \\
& \leqslant C 2^{-j^{\prime} d / 2} \sum_{k \geqslant \ell / 2-N_{0}} 2^{\left(j^{\prime}+k-\ell\right) d}\left(1+2^{j^{\prime}+k-\ell}\right)^{-N} 2^{-(k-\ell / 2) \rho} \\
& \leqslant C 2^{-j^{\prime} d / 2} 2^{\left(j^{\prime}-\ell / 2\right) \zeta^{\prime}}\left(1+2^{j^{\prime}-\ell / 2}\right)^{-N+d-\zeta^{\prime}}
\end{aligned}
$$

where $\zeta^{\prime}$ is any number $\zeta^{\prime}<\min [\rho, d]$. Then (3.2.29) and (3.2.30) show that (3.2.28) is smaller than

$$
C c_{j q} 2^{(j-\ell / 2)\left(\alpha+\zeta^{\prime}\right)} 2^{(j-\ell) \alpha^{\prime}}\left(1+2^{j-\ell / 2}\right)^{-N^{\prime}}
$$

for any $N^{\prime}$ i.e. the contribution of (3.2.18) is in $E_{\alpha+\zeta^{\prime}, \alpha^{\prime}, r}^{+\infty}$.

Let us now study the case $\frac{\alpha}{2}+\alpha^{\prime}<0, \alpha+\alpha^{\prime}+\frac{d}{2}+\nu>0$. We have

$$
\begin{aligned}
& \sum_{k \geqslant \ell / 2-N_{0}}\left\|\Delta_{j^{\prime}} g_{k} \Gamma_{\ell}(t)\right\|_{L^{\infty}\left(d t, L^{2}\right)} \\
\leqslant & C 2^{-j^{\prime} \frac{d}{2}} \sum_{k \geqslant \ell / 2-N_{0}} 2^{\left(j^{\prime}+k-\ell\right) d}\left(1+2^{j^{\prime}+k-\ell}\right)^{-N} 2^{-\left(k-\frac{\ell}{2}\right) \rho}\left[2^{k-\ell}\left(1+2^{k-\ell}\right)^{-1}\right]^{\nu} .
\end{aligned}
$$

When $j^{\prime}<\ell / 2$, we estimate the sum by

$$
\begin{aligned}
\sum_{k ; \ell / 2-N_{0} \leqslant k<\ell-j^{\prime}} 2^{\left(j^{\prime}+k-\ell\right) d} 2^{-(k-\ell / 2) \rho-j^{\prime} \nu}+\sum_{k ; k \geqslant \ell-j^{\prime}} 2^{-\left(j^{\prime}+k-\ell\right)(N-d)-(k-\ell / 2) \rho+(k-\ell) \nu} \\
\leqslant C 2^{\left(j^{\prime}-\ell / 2\right) \zeta^{\prime}-j^{\prime} \nu}
\end{aligned}
$$


with $\zeta^{\prime}<\min (\rho, d)$. When $j^{\prime}>\ell / 2$, the sum is smaller than

$$
\sum_{k ; k \geqslant \ell / 2-N_{0}} 2^{-\left(j^{\prime}+k-\ell\right)(N-d)-(k-\ell / 2) \rho+(k-\ell) \nu} \leqslant C 2^{-\left(j^{\prime}-\ell / 2\right) N^{\prime}-j^{\prime} \nu}
$$

for a convenient $N^{\prime}$. Consequently, (3.2.31) is smaller than

$$
C_{N^{\prime}} 2^{\left(j^{\prime}-\ell / 2\right) \zeta^{\prime}}\left(1+2^{j^{\prime}-\ell / 2}\right)^{-N} 2^{-j^{\prime}(\nu+d / 2)}
$$

for any $N$. If we use (3.1.19), we get also

$$
\left\|\phi_{q^{\prime}} S_{j^{\prime}-1} S_{k} w \Gamma_{\ell}(t)\right\|_{L^{p}\left(\frac{d t}{t}, L^{\infty}(d x)\right)} \leqslant C\left(1+2^{j^{\prime}-\ell / 2}\right)^{\mu_{0}} c_{j q}
$$

with $\left(c_{j q}\right)_{j q}$ in $\ell_{j}^{\infty} \ell_{q}^{r}$. Combining with (3.2.32), we obtain that (3.2.28) is smaller than

$$
C_{N} 2^{(j-\ell / 2)\left(\alpha+\zeta^{\prime}\right)+(j-\ell) \alpha^{\prime}}\left(1+2^{j-\ell / 2}\right)^{-N} c_{j q \ell}
$$

with $c_{j q \ell}=2^{\ell\left(\frac{\alpha}{2}+\alpha^{\prime}\right)} 2^{-j\left(\nu+\frac{d}{2}+\alpha+\alpha^{\prime}\right)} c_{j q}$. Since $\frac{\alpha}{2}+\alpha^{\prime}<0, \nu+\frac{d}{2}+\alpha+\alpha^{\prime}>0$, this sequence is smaller than a $\ell_{j}^{2} \ell_{q}^{r}$ sequence independent of $\ell$. This shows that contribution (3.2.18) is in $E_{\alpha+\zeta^{\prime}, \alpha^{\prime}, r}^{+\infty}$.

We have proved up to now that (3.2.7) belongs to $E_{\alpha+\zeta, \alpha^{\prime}, r}^{s+\rho, s^{\prime}} \subset E_{\alpha, \alpha^{\prime}, r}^{s+\rho, s^{\prime}}$. We still have to show that we can replace $E$ by $\widetilde{E}$.

When $\frac{\alpha}{2}+\alpha^{\prime}>0$, lemma 3.1.2 together with the assumption $\alpha^{\prime}>d / 2$, shows that these two spaces are identical. Let us assume now that $\frac{\alpha}{2}+\alpha^{\prime}<0$. By (3.2.8) we have to estimate the quantity

$$
\begin{aligned}
\phi_{q} \chi(D / \sqrt{t}) & \left(\sum_{k ; 2^{k}>c \sqrt{t}} S_{k-1} w g_{k}\right) \\
= & \sum_{k ; 2^{k}>c \sqrt{t}} \phi_{q} \chi(D / \sqrt{t})\left[\left(\chi(D / 10 \sqrt{t}) S_{k-1} w\right) g_{k}\right] \\
& \quad+\sum_{k ; 2^{k}>c \sqrt{t}} \phi_{q} \chi(D / \sqrt{t})\left[\left((1-\chi(D / 10 \sqrt{t})) S_{k-1} w\right) g_{k}\right]
\end{aligned}
$$

with $\chi \in C_{0}^{\infty}\left(\mathbb{R}^{d}\right)$, Supp $\chi \subset B(0,1), \chi \equiv 1$ on $B(0,1 / 2)$. The first term in the right hand side may be written

$$
\sum_{k} \sum_{q^{\prime}}\left(\phi_{q} \chi(D / \sqrt{t}) \widetilde{\phi}_{q^{\prime}}\right)\left(\left(\phi_{q^{\prime}} \chi(D / 10 \sqrt{t}) S_{k-1} w\right) g_{k}\right) .
$$

Since $w \in \widetilde{E}_{\alpha, \alpha^{\prime}, r}^{s, s^{\prime}}$, we have, by lemma 3.1.2

$$
\left\|\phi_{q^{\prime}} \chi(D / 10 \sqrt{t}) S_{k-1} w\right\|_{L^{\infty}(d t d x)} \leqslant C c_{q^{\prime}}
$$

for a constant $C$ and a $\ell_{q^{\prime}}^{r}$ sequence $\left(c_{q^{\prime}}\right)_{q^{\prime}}$ independent of $k$. Moreover, by (3.2.11) and the assumption $\rho-\mu>0, \sum_{k ; 2^{k}>c \sqrt{t}}\left\|g_{k}\right\|_{L^{\infty}(d t d x)} \leqslant C$. We thus deduce from 
(3.2.35), (3.2.36) that the first term in the right hand side of (3.2.34) has $L^{\infty}$ norm in $\ell_{q}^{r}$. The last sum in (3.2.34) may be written

$$
\sum_{k} \sum_{q^{\prime}}\left(\phi_{q} \chi(D / \sqrt{t}) \widetilde{\phi}_{q^{\prime}}\right)\left[\left(\phi_{q^{\prime}}(1-\chi)(D / 10 \sqrt{t}) S_{k-1} w\right)(1-\chi)(D / \sqrt{t}) g_{k}\right] .
$$

as follows from inspection of the supports of the Fourier transforms. We estimate

$$
\begin{aligned}
& \left\|\left(\phi_{q^{\prime}}(1-\chi)(D / 10 \sqrt{t})\right) S_{k-1} w(t, \cdot)\right\|_{L^{\infty}(d x)} \\
& \quad \leqslant \sum_{q^{\prime \prime}} \sum_{k^{\prime} ; c \sqrt{t}<2^{k^{\prime}}<2^{k-1}} C_{N}\left\langle q^{\prime}-q^{\prime \prime}\right\rangle^{-N} 2^{k^{\prime} d / 2}\left\|\phi_{q^{\prime \prime}} \Delta_{k^{\prime}} w(t, \cdot)\right\|_{L^{2}} .
\end{aligned}
$$

When $2^{k}<t$ we get an upper bound

$$
\sum_{k^{\prime} ; c \sqrt{t}<2^{k^{\prime}}<2^{k-1}} 2^{k^{\prime} d / 2}\left(\frac{2^{k^{\prime}}}{\sqrt{t}}\right)^{-s}\left(\frac{2^{k^{\prime}}}{t}\right)^{\alpha^{\prime}} c_{k^{\prime} q^{\prime}} \leqslant \sum_{k^{\prime} ; c \sqrt{t}<2^{k^{\prime}}<2^{k-1}} 2^{k^{\prime}\left(d / 2-\alpha^{\prime}\right)}\left(\frac{2^{k^{\prime}}}{\sqrt{t}}\right)^{\mu_{0}} c_{k^{\prime} q^{\prime}}
$$

where $\left(c_{k^{\prime} q^{\prime}}\right)_{k^{\prime} q^{\prime}}$ is in $\ell_{k^{\prime}}^{2} \ell_{q^{\prime}}^{r}$ and $\mu_{0}$ is chosen large enough. If $\mu_{0}+\frac{d}{2}-\alpha^{\prime}>0$, we get an upper bound of type

$$
C 2^{k\left(d / 2-\alpha^{\prime}\right)} c_{k q^{\prime}}^{\prime}\left(\frac{2^{k}}{\sqrt{t}}\right)^{\mu_{0}} \leqslant C \widetilde{c}_{q^{\prime}}\left(\frac{2^{k}}{\sqrt{t}}\right)^{\mu_{0}}
$$

where $\left(c_{k q^{\prime}}^{\prime}\right)_{k q^{\prime}} \in \ell_{k}^{2} \ell_{q^{\prime}}^{r},\left(\widetilde{c}_{q^{\prime}}\right)_{q^{\prime}} \in \ell^{r}$ independent of $k, t$.

When $2^{k}>t$, we control (3.2.38) by

$$
\sum_{k^{\prime} ; c \sqrt{t}<2^{k^{\prime}}<t} 2^{k^{\prime}\left(d / 2-\alpha^{\prime}\right)}\left(\frac{2^{k^{\prime}}}{\sqrt{t}}\right)^{\mu_{0}} c_{k^{\prime} q^{\prime}}+\sum_{k^{\prime} ; t<2^{k^{\prime}}<2^{k-1}} 2^{k^{\prime} d / 2}\left(\frac{2^{k^{\prime}}}{\sqrt{t}}\right)^{-s}\left(\frac{2^{k^{\prime}}}{t}\right)^{-s^{\prime}} c_{k^{\prime} q^{\prime}}
$$

which is also controlled by $C \widetilde{c}_{q^{\prime}}\left(\frac{2^{k}}{\sqrt{t}}\right)^{\mu_{0}}$. We thus have

$$
\left\|\phi_{q^{\prime}}(1-\chi)(D / 10 \sqrt{t}) S_{k-1} w(t, \cdot)\right\|_{L^{\infty}(d x)} \leqslant C \widetilde{c}_{q^{\prime}}\left(\frac{2^{k}}{\sqrt{t}}\right)^{\mu_{0}}
$$

for some $\mu_{0}$ and some $\ell_{q^{\prime}}^{r}$ sequence $\left(\widetilde{c}_{q^{\prime}}\right)_{q^{\prime}}$. Moreover we deduce from (3.2.10)

$$
\left\|(1-\chi)(D / \sqrt{t}) g_{k}\right\|_{L^{\infty}(d x)} \leqslant C \sum_{2^{j}>c \sqrt{t}} 2^{j d / 2}\left\|\Delta_{j} g_{k}\right\|_{L^{2}(d x)} \leqslant C_{N}\left(2^{k} / \sqrt{t}\right)^{-N} .
$$

We deduce from these two inequalities that the $L^{\infty}(d x d t)$ norm of (3.2.37) is smaller than a $\ell_{q}^{r}$ sequence. This shows that the $L^{\infty}(d x d t)$ norm of (3.2.34) is in $\ell_{q}^{r}$ and concludes the proof of proposition 3.2.2.

Our next objective is to estimate quantities of type

$$
T_{a(t, x) w} b(t, D) e^{i m \theta}
$$

where $m \in \mathbb{Z}^{*}, a(t, x)$ is a smooth function of $x$ satisfying for any $\gamma \in \mathbb{N}^{d}$

$$
\left|\left(D_{x} / \sqrt{t}\right)^{\gamma} a(t, x)\right| \leqslant C_{\gamma}(1+\sqrt{t}|x|)^{m_{1}-|\gamma|}(1+|x|)^{m_{1}^{\prime}}
$$


and where $b(t, \xi)$ is in $\Sigma_{\nu}^{m_{2}}$, using the notations of definition 3.2.1.

Proposition 3.2.4. - Let $m_{1}, m_{1}^{\prime}, m_{2}, \nu$ be real numbers satisfying the inequalities $m_{1} \leqslant 0, m_{1}^{\prime} \leqslant 0$, and let $a$ be a symbol verifying (3.2.40) and $b \in \Sigma_{\nu}^{m_{2}}$.

(i) Take $s, s^{\prime}, \alpha, \alpha^{\prime}, r$ such that $s, s^{\prime}, \alpha, \alpha^{\prime} \in \mathbb{R}, r \in[1,+\infty]$,

$$
-d<\alpha+\alpha^{\prime}<-\frac{d}{2}, \quad s<\alpha+2 \alpha^{\prime}, \quad s+s^{\prime}<-1 / 2, \quad \nu=\frac{d}{2} .
$$

Let $w \in E_{\alpha, \alpha^{\prime}, r}^{s, s^{\prime}}$ be such that $a(t, x) w \in E_{\alpha, \alpha^{\prime}, r}^{s, s^{\prime}}$. Then (3.2.39) belongs to $E_{\alpha+2 d, \alpha^{\prime}, r}^{s-m_{1}-s^{\prime}-m_{1}^{\prime}}$.

(ii) Assume instead of (3.2.41),

$$
-\frac{d}{2}<\alpha+\alpha^{\prime}, \quad s<\alpha^{\prime}-\frac{d}{2}, \quad s+s^{\prime}<-\frac{1}{2}, \quad \nu=0 .
$$

Then if $w \in E_{\alpha, \alpha^{\prime}, r}^{s, s^{\prime}}$ and $a(t, x) w \in E_{\alpha, \alpha^{\prime}, r}^{s, s^{\prime}}$, (3.2.39) belongs to the space $E_{\alpha+d, \alpha^{\prime}, r}^{s-m_{2}, s^{\prime}-m_{1}^{\prime}}$.

We can in the above statements replace operator $T$ by operator $\widetilde{T}$ of (3.1.16).

We first prove the following lemmas:

LEMma 3.2.5. - Let a be a symbol satisfying (3.2.40) with $m_{1} \leqslant 0, m_{1}^{\prime} \leqslant 0, b \in$ $\Sigma_{\nu}^{m_{2}}, m \in \mathbb{Z}^{*}$. We have the following estimates

$$
\left\|a(t, x) \Delta_{j} b(t, D) e^{i m \theta}\right\|_{L^{2}(d x)} \leqslant C\left(\frac{2^{j}}{t}\right)^{\nu+d / 2}\left(1+\frac{2^{j}}{t}\right)^{-\nu+m_{1}^{\prime}}\left(1+\frac{2^{j}}{\sqrt{t}}\right)^{m_{1}+m_{2}}
$$

$$
\left\|a(t, x) \Delta_{j} b(t, D) e^{i m \theta}\right\|_{L^{\infty}(d x)} \leqslant C\left(\frac{2^{j}}{t}\right)^{\nu}\left(1+\frac{2^{j}}{t}\right)^{-\nu+m_{1}^{\prime}}\left(1+\frac{2^{j}}{\sqrt{t}}\right)^{m_{1}+m_{2}} .
$$

Proof. - Remark first that since $m_{1} \leqslant 0, m_{1}^{\prime} \leqslant 0,(3.2 .40)$ implies that

$$
\left\|\mathbb{1}_{\left\{|x| \geqslant c 2^{j} / t\right\}} a(t, x)\right\|_{L^{\infty}} \leqslant C\left(1+\frac{2^{j}}{\sqrt{t}}\right)^{m_{1}}\left(1+\frac{2^{j}}{t}\right)^{m_{1}^{\prime}} .
$$

Moreover, a direct computation and the assumption $b(t, \xi) \in \Sigma_{\nu}^{m_{2}}$ shows that

$$
\begin{gathered}
\left\|\Delta_{j} b(t, D) e^{i m \theta}\right\|_{L^{2}(d x)} \leqslant C\left(\frac{2^{j}}{t}\right)^{\nu+d / 2}\left(1+\frac{2^{j}}{t}\right)^{-\nu}\left(1+\frac{2^{j}}{\sqrt{t}}\right)^{m_{2}} \\
\left\|\Delta_{j} b(t, D) e^{i m \theta}\right\|_{L^{\infty}(d x)} \leqslant C\left(\frac{2^{j}}{t}\right)^{\nu}\left(1+\frac{2^{j}}{t}\right)^{-\nu}\left(1+\frac{2^{j}}{\sqrt{t}}\right)^{m_{2}} .
\end{gathered}
$$

Together with (3.2.45), these inequalities show that (3.2.43), (3.2.44) hold true if we replace in the left hand side $a(t, x)$ by $\mathbb{1}_{\left\{|x| \geqslant c 2^{j} / t\right\}} a(t, x)$. Consequently, we are left with estimating in $L^{2}$ or $L^{\infty}$ the quantity

$$
\left|\mathbb{1}_{\left\{|x|<c 2^{j} / t\right\}} a(t, x) \Delta_{j} b(t, D) e^{i m \theta}\right| \leqslant C\left|\mathbb{1}_{\left\{|x|<c 2^{j} / t\right\}} \Delta_{j} b(t, D) e^{i m \theta}\right|,
$$


where we used that $a$ is bounded since $m_{1} \leqslant 0, m_{1}^{\prime} \leqslant 0$. When $2^{j}<\sqrt{t}$, the wanted upper bound follows from the above estimate of the $L^{2}$ or $L^{\infty}$ norms of $\Delta_{j} b(t, D) e^{i m \theta}$. When $2^{j} \geqslant \sqrt{t}$, we write $\Delta_{j} b(t, D) e^{i m \theta}$ as a constant multiple of

$$
\int e^{i x \xi-i \xi^{2} / m t} \varphi\left(2^{-j} \xi\right) b(t, \xi) \frac{d \xi}{t^{d / 2}}=\left(\frac{2^{j}}{\sqrt{t}}\right)^{d} \int e^{i 2^{j}\left[x \xi-\frac{2^{j} \xi^{2}}{m t}\right] \widetilde{b}_{j}}(t, \xi) d \xi
$$

where $\widetilde{b}_{j}(t, \xi)=\varphi(\xi) b\left(t, 2^{j} \xi\right)$ is supported in a fixed ring of size 1 and satisfies by (3.2.2) for any $\gamma \in \mathbb{N}^{d}$

$$
\left|\partial_{\xi}^{\gamma} \widetilde{b}_{j}(t, \xi)\right| \leqslant C_{\gamma}\left(\frac{2^{j}}{t}\right)^{\nu}\left(1+\frac{2^{j}}{t}\right)^{-\nu}\left(1+\frac{2^{j}}{\sqrt{t}}\right)^{m_{2}} .
$$

Define the operator

$$
L_{j}\left(t, x, \xi, D_{\xi}\right)=\left[1+2^{2 j}\left(x-2^{j+1} \frac{\xi}{m t}\right)^{2}\right]^{-1}\left[1+2^{j}\left(x-2^{j+1} \frac{\xi}{m t}\right) \cdot D_{\xi}\right] .
$$

Remark that when $\xi \in \operatorname{Supp} \varphi$ and $x$ stays in the domain $|x| \leqslant c 2^{j} / t$ with $c$ small enough, this operator is of form $L_{j}=\alpha_{j}^{0}(t, x, \xi)+\alpha_{j}^{1}(t, x, \xi) \cdot D_{\xi}$ where $\alpha_{j}^{0}, \alpha_{j}^{1}$ are smooth coefficients satisfying for $\ell=0,1$ and $\gamma \in \mathbb{N}^{d}$

$$
\left|\partial_{\xi}^{\gamma} \alpha_{j}^{\ell}(t, x, \xi)\right| \leqslant C_{\gamma}\left(1+2^{j} / \sqrt{t}\right)^{-2} .
$$

Consequently, ${ }^{t} L_{j}$ is of the same type, and integrating by parts, we write (3.2.48) as

$$
\left(\frac{2^{j}}{\sqrt{t}}\right)^{d} \int e^{i 2^{j}\left[x \xi-\frac{2^{j} \xi^{2}}{m t}\right]}\left({ }^{t} L_{j}\right)^{M} \widetilde{b}_{j}(t, \xi) d \xi
$$

when $x$ stays in the domain $\left\{|x|<c 2^{j} / t\right\}$, for any $M \in \mathbb{N}$. It follows then from (3.2.49), (3.2.51) that (3.2.47) is smaller than

$$
C_{N} \mathbb{1}_{\left\{|x|<c 2^{j} / t\right\}}\left(\frac{2^{j}}{t}\right)^{\nu}\left(1+\frac{2^{j}}{\sqrt{t}}\right)^{-N}
$$

for any $N$. This implies immediately an upper bound of the $L^{\infty}$ (resp. $L^{2}$ ) norm of this quantity by the right hand side of (3.2.44) (resp. (3.2.43)) and concludes the proof.

Lemma 3.2.6. - If $s^{\prime}$ is a real number, define $s_{2}^{\prime}=s^{\prime}+\frac{1}{2}, s_{\infty}^{\prime}=s^{\prime}$. For $r \in[1,+\infty]$, set $r_{2}=r, r_{\infty}=\widetilde{r}$, with $\widetilde{r}$ defined in definition 3.1.\%.

(i) Let $s, s^{\prime}, \alpha, \alpha^{\prime}, r$ be real numbers, with $r \in[1,+\infty]$, and assume that

$$
-d<\alpha+\alpha^{\prime}<-d / 2, \quad s<\alpha+2 \alpha^{\prime}, \quad s+s^{\prime}<-1 / 2 .
$$

If $w \in E_{\alpha, \alpha^{\prime}, r}^{s, s^{\prime}}$, one has the following estimates for $p=2$ or $p=\infty$ :

$$
\left\|\phi_{q}(x) \chi(D / \sqrt{t}) w \Gamma_{\ell}(t)\right\|_{L^{p}\left(\frac{d t}{t}, L^{\infty}\right)} \leqslant C 2^{-s(j-\ell / 2)+(j-\ell)\left(\alpha^{\prime}-d\right)} c_{j q}
$$

when $\ell / 2 \leqslant j \leqslant \ell$,

$$
\left\|\phi_{q}(x) S_{j} w \Gamma_{\ell}(t)\right\|_{L^{p}\left(\frac{d t}{t}, L^{\infty}\right)} \leqslant C 2^{-\frac{\ell}{2} \alpha-\ell \alpha^{\prime}} c_{q}
$$


when $j \leqslant \ell / 2$,

$$
\left\|\phi_{q}(x) \chi(D / \sqrt{t}) w \Gamma_{\ell}(t)\right\|_{L^{p}\left(\frac{d t}{t}, L^{2}\right)} \leqslant C 2^{-s(j-\ell / 2)-s_{p}^{\prime}(j-\ell)} c_{j q}
$$

when $\ell \leqslant j$,

$$
\begin{aligned}
\| \phi_{q}(x)(1-\chi(D / \sqrt{t})) S_{j} w \Gamma_{\ell}(t) & \|_{L^{p}\left(\frac{d t}{t}, L^{2}\right)} \\
& \leqslant C 2^{-s(j-\ell / 2)+(j-\ell) \alpha^{\prime}}\left(1+2^{j-\ell}\right)^{-\alpha^{\prime}-s_{p}^{\prime}} c_{j q}
\end{aligned}
$$

when $\ell / 2 \leqslant j$, where $\left(c_{j q}\right)_{j q}$ (resp. $\left.\left(c_{q}\right)_{q}\right)$ is a sequence in the unit ball of $\ell_{j}^{2} \ell_{q}^{r_{p}}$ (resp. $\left.\ell_{q}^{r_{p}}\right)$.

(ii) Assume instead of (3.2.53)

$$
-d / 2<\alpha+\alpha^{\prime}, \quad s<\alpha^{\prime}-d / 2, \quad s+s^{\prime}<-1 / 2 .
$$

If $w \in E_{\alpha, \alpha^{\prime}, r}^{s, s^{\prime}}$, one has for $p=2$ or $p=\infty$,

$$
\left\|\phi_{q}(x) \chi(D / \sqrt{t}) w \Gamma_{\ell}(t)\right\|_{L^{p}\left(\frac{d t}{t}, L^{\infty}\right)} \leqslant C 2^{-s(j-\ell / 2)+(j-\ell)\left(\alpha^{\prime}-d / 2\right)} c_{j q}
$$

when $\ell / 2 \leqslant j \leqslant \ell$,

$$
\left\|\phi_{q}(x) S_{j} w \Gamma_{\ell}(t)\right\|_{L^{p}\left(\frac{d t}{t}, L^{\infty}\right)} \leqslant C 2^{\left(j-\frac{\ell}{2}\right) \alpha+(j-\ell) \alpha^{\prime}} c_{j q} 2^{j \frac{d}{2}}
$$

when $j \leqslant \ell / 2$, and (3.2.56), (3.2.57) when respectively $\ell \leqslant j$ and $\ell / 2 \leqslant j$, for some sequence $\left(c_{j q}\right)_{j q}$ in the unit ball of $\ell_{j}^{2} \ell_{q}^{r}$.

Proof. - Let us prove first (3.2.54) and (3.2.55). We estimate, with some $N_{1} \in \mathbb{N}$,

$$
\begin{aligned}
\| \phi_{q}(x) \chi & (D / \sqrt{t}) w \Gamma_{\ell}(t) \|_{L^{p}\left(\frac{d t}{t}, L^{\infty}\right)} \\
& \leqslant \sum_{q^{\prime}} \sum_{j^{\prime} ; j^{\prime} \leqslant \ell / 2+N_{1}}\left\|\left(\phi_{q}(x) \chi(D / \sqrt{t}) \widetilde{\phi}_{q^{\prime}}\right)\left(\phi_{q^{\prime}} \Delta_{j^{\prime}} w \Gamma_{\ell}(t)\right)\right\|_{L^{p}\left(\frac{d t}{t}, L^{\infty}\right)} \\
& \leqslant \sum_{q^{\prime}} \sum_{j^{\prime} ; j^{\prime} \leqslant \ell / 2+N_{1}} C_{N} 2^{j^{\prime} d / 2}\left\langle q-q^{\prime}\right\rangle^{-N}\left\|\phi_{q^{\prime}} \Delta_{j^{\prime}} w \Gamma_{\ell}(t)\right\|_{L^{p}\left(\frac{d t}{t}, L^{2}\right)} .
\end{aligned}
$$

Using the definition of $E_{\alpha, \alpha^{\prime}, r}^{s, s^{\prime}}$, we get the upper bound

$$
C_{N} \sum_{q^{\prime}} \sum_{j^{\prime} ; j^{\prime} \leqslant \ell / 2+N_{1}} 2^{j^{\prime} d / 2}\left\langle q-q^{\prime}\right\rangle^{-N} 2^{\alpha\left(j^{\prime}-\ell / 2\right)+\alpha^{\prime}\left(j^{\prime}-\ell\right)} c_{j^{\prime} q^{\prime}}
$$

for a $\ell_{j^{\prime}}^{2} \ell_{q^{\prime}}^{r_{p}}$ sequence $\left(c_{j^{\prime} q^{\prime}}\right)_{j^{\prime} q^{\prime}}$. In case of assertion (i) of the lemma, under assumption (3.2.53), (3.2.62) is smaller than $C 2^{-\frac{\ell}{2} \alpha-\ell \alpha^{\prime}} c_{q}^{\prime}$ for a $\ell_{q}^{r_{p}}$ sequence $\left(c_{q}^{\prime}\right)_{q}$. This last expression may be written

$$
C 2^{-s(j-\ell / 2)+(j-\ell)\left(\alpha^{\prime}-d\right)} c_{j q \ell}
$$

with

$$
c_{j q \ell}=2^{j\left(s-\alpha^{\prime}+d\right)} 2^{\ell(-s / 2-d-\alpha / 2)} c_{q}^{\prime} .
$$

This quantity is smaller than $2^{-j\left(\alpha+\alpha^{\prime}+d\right)} c_{q}^{\prime}$ when $s / 2+d+\alpha / 2 \leqslant 0, \ell / 2 \leqslant j \leqslant \ell$, and than $2^{j\left(s / 2-\alpha^{\prime}-\alpha / 2\right)} c_{q}^{\prime}$ when $s / 2+d+\alpha / 2 \geqslant 0, \ell / 2 \leqslant j \leqslant \ell$. In both cases, because 
of (3.2.53), we get an upper bound by a $\ell_{j}^{2} \ell_{q}^{r_{p}}$ sequence, whence (3.2.54). One proves $(3.2 .55)$ in the same way.

Let us now prove inequality (3.2.59) of assertion (ii) of the lemma. Because of (3.2.58), the quantity (3.2.62) is smaller than $C 2^{\frac{\ell}{2}\left(\frac{d}{2}-\alpha^{\prime}\right)} c_{\ell q}$ with $\left(c_{\ell q}\right)_{\ell q} \in \ell_{\ell}^{2} \ell_{q}^{r}$. We write this upper bound $C 2^{-s(j-\ell / 2)+(j-\ell)\left(\alpha^{\prime}-d / 2\right)} c_{j q \ell}$ with

$$
c_{j q \ell}=2^{(j-\ell / 2)\left(s-\alpha^{\prime}+d / 2\right)} c_{\ell q} .
$$

Since $s-\alpha^{\prime}+d / 2<0$, the supremum for $\ell \leqslant 2 j$ of this sequence is in $\ell_{j}^{2} \ell_{q}^{r}$. This gives the conclusion (3.2.59). One gets (3.2.60) by a similar computation.

Let us prove now (3.2.56). Arguing as in (3.2.61) we write

$$
\begin{aligned}
& \left\|\phi_{q}(x) \chi(D / \sqrt{t}) w \Gamma_{\ell}(t)\right\|_{L^{p}\left(\frac{d t}{t}, L^{2}\right)} \\
& \leqslant C_{N} \sum_{q^{\prime}} \sum_{j^{\prime} ; j^{\prime} \leqslant \ell / 2+N_{1}}\left\langle q-q^{\prime}\right\rangle^{-N} 2^{\alpha\left(j^{\prime}-\ell / 2\right)+\alpha^{\prime}\left(j^{\prime}-\ell\right)} c_{j^{\prime} q^{\prime}} .
\end{aligned}
$$

Let us consider first the case $\alpha+\alpha^{\prime} \leqslant 0$. Then the right hand side of (3.2.63) is smaller than $C 2^{-\frac{\ell}{2} \alpha-\ell \alpha^{\prime}+\ell \varepsilon} c_{q}^{\prime}$ where $\left(c_{q}^{\prime}\right)_{q} \in \ell_{q}^{r}, \varepsilon=0$ if $\alpha+\alpha^{\prime}<0, \varepsilon>0$ arbitrarily small if $\alpha+\alpha^{\prime}=0$. We write this quantity $C 2^{-(j-\ell / 2) s-(j-\ell) s_{p}^{\prime}} c_{j q \ell}$ where

$$
c_{j q \ell}=2^{j\left(s+s_{p}^{\prime}\right)} 2^{-\frac{\ell}{2}\left(\alpha+2 \alpha^{\prime}+s+2 s_{p}^{\prime}-2 \varepsilon\right)} c_{q}^{\prime} \leqslant 2^{j(\varepsilon+\delta)} c_{q}^{\prime}
$$

if $\ell \leqslant j$ and $\delta=\max \left[s+s_{p}^{\prime}, \frac{s}{2}-\frac{\alpha}{2}-\alpha^{\prime}\right]$. The assumptions (3.2.53) or (3.2.58) imply that $\delta<0$, so the right hand side of (3.2.64) is in $\ell_{j}^{2} \ell_{q}^{r}$ if $\varepsilon<-\delta$, whence (3.2.56) in this case. When $\alpha+\alpha^{\prime}>0$ we are necessarily in case (ii) and the right hand side of (3.2.63) is smaller than $c 2^{-\frac{\ell}{2} \alpha^{\prime}} c_{\ell q}^{\prime}$ for a $\ell_{\ell}^{2} \ell_{q}^{r}$ sequence $\left(c_{\ell q}^{\prime}\right)_{\ell q}$. We write this quantity $2^{-s(j-\ell / 2)-s_{p}^{\prime}(j-\ell)} c_{j q \ell}$, with for $\ell \leqslant j$

$$
c_{j q \ell}=2^{j\left(s+s_{p}^{\prime}\right)} 2^{-\frac{\ell}{2}\left[s+2 s_{p}^{\prime}+\alpha^{\prime}\right]} c_{\ell q}^{\prime} \leqslant 2^{j \max \left[s+s_{p}^{\prime}, \frac{s}{2}-\frac{\alpha^{\prime}}{2}\right]} c_{\ell q}^{\prime} .
$$

Since by (3.2.58) $s+s_{p}^{\prime}<0, s<\alpha^{\prime}$, the supremum in $\ell \leqslant j$ of this last sequence belongs to $\ell_{j}^{2} \ell_{q}^{r}$. This concludes the proof of (3.2.56).

Finally, let us prove (3.2.57) in cases (i) and (ii). We write

$$
\begin{aligned}
& \left\|\phi_{q}(x)(1-\chi)(D / \sqrt{t}) S_{j} w \Gamma_{\ell}(t)\right\|_{L^{p}\left(\frac{d t}{t}, L^{2}\right)} \\
& \leqslant \sum_{q^{\prime}} \sum_{j^{\prime} ; \ell / 2+N_{1} \leqslant j^{\prime} \leqslant j-1}\left\|\left(\phi_{q}(1-\chi)(D / \sqrt{t}) \widetilde{\phi}_{q^{\prime}}\right)\left(\phi_{q^{\prime}} \Delta_{j^{\prime}} w \Gamma_{\ell}(t)\right)\right\|_{L^{p}\left(\frac{d t}{t}, L^{2}\right)} \\
& \leqslant C_{N} \sum_{q^{\prime}}\left(\sum_{j^{\prime} ; \ell / 2+N_{1} \leqslant j^{\prime} \leqslant \inf (\ell, j-1)}\left\langle q-q^{\prime}\right\rangle^{-N} 2^{\left(j^{\prime}-\ell\right) \alpha^{\prime}-s\left(j^{\prime}-\ell / 2\right)} c_{j^{\prime} q^{\prime}}\right. \\
& \left.\quad+\sum_{j^{\prime} ; \ell<j^{\prime}<j}\left\langle q-q^{\prime}\right\rangle^{-N} 2^{-s\left(j^{\prime}-\ell / 2\right)-s_{p}^{\prime}\left(j^{\prime}-\ell\right)} c_{j^{\prime} q^{\prime}}\right)
\end{aligned}
$$


where $\left(c_{j^{\prime} q^{\prime}}\right)_{j^{\prime} q^{\prime}} \in \ell_{j^{\prime}}^{2} \ell_{q^{\prime}}^{r}$ and where we used the definition of $E_{\alpha, \alpha^{\prime}, r}^{s, s^{\prime}}$. When $j-1 \leqslant \ell$, since in both cases (i) and (ii) $\alpha^{\prime}-s>0$, we get for (3.2.65) the upper bound

$$
C 2^{(j-\ell) \alpha^{\prime}} 2^{-s(j-\ell / 2)} c_{j q}^{\prime}
$$

for a $\ell_{j}^{2} \ell_{q}^{r}$ sequence $\left(c_{j q}^{\prime}\right)_{j q}$. When $\ell<j-1$, using that $s+s_{p}^{\prime}<0, \alpha^{\prime}-s>0$ we obtain the upper bound

$$
C 2^{-s(j-\ell / 2)-s_{p}^{\prime}(j-\ell)}\left[c_{j q}^{\prime}+c_{\ell q}^{\prime \prime} 2^{\left(s+s_{p}^{\prime}\right)(j-\ell)}\right]
$$

for a $\ell_{j}^{2} \ell_{q}^{r}\left(\right.$ resp. $\left.\ell_{\ell}^{2} \ell_{q}^{r}\right)$ sequence $\left(c_{j q}^{\prime}\right)_{j q}$ (resp. $\left.\left(c_{\ell q}^{\prime \prime}\right)_{\ell q}\right)$. The conclusion follows from these estimates.

Proof of proposition 3.2.4. - We want to estimate

$$
\phi_{q} \Delta_{j}\left[T_{a(t, x) w} b(t, D) e^{i m \theta}\right] \Gamma_{\ell}(t)
$$

in $L^{2}\left(\frac{d t}{t}, L^{2}(d x)\right)$ and $L^{\infty}\left(d t, L^{2}(d x)\right)$. We study successively the following cases.

Estimate for $j \leqslant \ell / 2$. - We write for $\left|j-j^{\prime}\right| \leqslant N_{0}, N_{0}$ a fixed integer, and $p=2$ or $p=\infty$

$$
\begin{aligned}
& \left\|\phi_{q} \Delta_{j}\left[S_{j^{\prime}-1}(a(t, x) w) \Gamma_{\ell}(t) \Delta_{j^{\prime}} b(t, D) e^{i m \theta}\right]\right\|_{L^{p}\left(\frac{d t}{t}, L^{2}\right)} \\
& \leqslant \sum_{q^{\prime}} C_{N}\left\langle q-q^{\prime}\right\rangle^{-N} \| \phi_{q^{\prime}} S_{j^{\prime}-1}[a(t, x) w] \\
& \Gamma_{\ell}(t) \|_{L^{p}\left(\frac{d t}{t}, L^{\infty}\right)} \\
& \times\left\|\Delta_{j^{\prime}}\left[b(t, D) e^{i m \theta}\right]\right\|_{L^{\infty}\left(L^{2}\right)} .
\end{aligned}
$$

In case (i), we use inequality (3.2.55) (for aw instead of $w$ ) together with (3.2.43) with $\nu=d / 2$. We get the upper bound

$$
C 2^{-\frac{\ell}{2} \alpha-\ell \alpha^{\prime}} c_{q} 2^{(j-\ell) d}=C 2^{(j-\ell / 2)(\alpha+2 d)} 2^{(j-\ell) \alpha^{\prime}} c_{j q}
$$

with $\left(c_{q}\right)_{q} \in \ell_{q}^{r_{p}}$ and $c_{j q}=2^{-j\left(\alpha+\alpha^{\prime}+d\right)} c_{q}$, a sequence in $\ell_{j}^{2} \ell_{q}^{r_{p}}$, as we have $\alpha+\alpha^{\prime}+d>0$ by (3.2.41). In case (ii), we apply (3.2.60) together with (3.2.43) with $\nu=0$. We get the upper bound

$$
C 2^{(j-\ell / 2) \alpha+(j-\ell) \alpha^{\prime}} c_{j q} 2^{j d / 2} 2^{(j-\ell) d / 2}=C 2^{(j-\ell / 2)(\alpha+d)+(j-\ell) \alpha^{\prime}} c_{j q}
$$

for a $\ell_{j}^{2} \ell_{q}^{r_{p}}$ sequence $\left(c_{j q}\right)_{j q}$. The expressions (3.2.68), (3.2.69) show that (3.2.66) belongs to the spaces indicated in the statement of the proposition.

Estimate for $\ell / 2<j \leqslant \ell$. - For $j^{\prime}$ with $\left|j-j^{\prime}\right|$ smaller than some given integer $N_{1}$, we write

$$
S_{j^{\prime}-1}(a w) \Delta_{j^{\prime}}\left(b(t, D) e^{i m \theta}\right)=U_{j^{\prime}} \cdot V_{j^{\prime}}
$$

where, if $c(t, x)=\left(1+t x^{2}\right)^{-m_{1} / 2}\left(1+x^{2}\right)^{-m_{1}^{\prime} / 2}$, we set

$$
\begin{aligned}
U_{j^{\prime}} & =c(t, x) S_{j^{\prime}-1}(a w) \\
V_{j^{\prime}} & =c(t, x)^{-1} \Delta_{j^{\prime}}\left[b(t, D) e^{i m \theta}\right] .
\end{aligned}
$$


We further decompose, choosing $N_{2}$ such that $j^{\prime \prime} \geqslant j^{\prime}+N_{2}$ and $j \geqslant \ell / 2$ imply $(1-\chi)(D / \sqrt{t}) \Delta_{j^{\prime \prime}} \Gamma_{\ell}=\Delta_{j^{\prime \prime}} \Gamma_{\ell}$,

$$
\begin{aligned}
U_{j^{\prime}} & =U_{j^{\prime}}^{\prime}+U_{j^{\prime}}^{\prime \prime}+\sum_{j^{\prime \prime} \geqslant j^{\prime}+N_{2}} U_{j^{\prime}}^{j^{\prime \prime}} \\
U_{j^{\prime}}^{\prime} & =c(t, x) S_{j^{\prime}-1}[a \chi(D / \sqrt{t}) w] \\
U_{j^{\prime}}^{\prime \prime} & =c(t, x) S_{j^{\prime}-1}\left[a(1-\chi)(D / \sqrt{t}) S_{j^{\prime}+N_{2}} w\right] \\
U_{j^{\prime}}^{j^{\prime \prime}} & =c(t, x) S_{j^{\prime}-1}\left[a \Delta_{j^{\prime \prime}} w\right]
\end{aligned}
$$

when $t$ is in the support of $\Gamma_{\ell}(t)$. We have the estimate

$$
\begin{aligned}
& \left\|\phi_{q}(x) U_{j^{\prime}} V_{j^{\prime}} \Gamma_{\ell}(t)\right\|_{L^{p}\left(\frac{d t}{t}, L^{2}(d x)\right)} \\
& \leqslant\left\|\phi_{q}(x) U_{j^{\prime}}^{\prime} \Gamma_{\ell}(t)\right\|_{L^{p}\left(\frac{d t}{t}, L^{\infty}(d x)\right)}\left\|V_{j^{\prime}} \Gamma_{\ell}(t)\right\|_{L^{\infty}\left(d t, L^{2}(d x)\right)} \\
& \quad+\left\|\phi_{q}(x) U_{j^{\prime}}^{\prime \prime} \Gamma_{\ell}(t)\right\|_{L^{p}\left(\frac{d t}{t}, L^{2}(d x)\right)}\left\|V_{j^{\prime}} \Gamma_{\ell}(t)\right\|_{L^{\infty}(d t d x)} \\
& \quad+\sum_{j^{\prime \prime} \geqslant j^{\prime}}\left\|\phi_{q}(x) U_{j^{\prime}}^{j^{\prime \prime}} \Gamma_{\ell}(t)\right\|_{L^{p}\left(\frac{d t}{t}, L^{2}(d x)\right)}\left\|V_{j^{\prime}} \Gamma_{\ell}(t)\right\|_{L^{\infty}(d t d x)} .
\end{aligned}
$$

We write

$$
\begin{aligned}
\phi_{q} U_{j^{\prime}}^{\prime} & =\sum_{q^{\prime}} K_{j^{\prime} q q^{\prime}}\left[\phi_{q^{\prime}} \chi(D / \sqrt{t}) w\right] \\
\phi_{q} U_{j^{\prime}}^{\prime \prime} & =\sum_{q^{\prime}} K_{j^{\prime} q q^{\prime}}\left[\phi_{q^{\prime}}(1-\chi)(D / \sqrt{t}) S_{j^{\prime}+N_{2}} w\right] \\
\phi_{q} U_{j^{\prime}}^{j^{\prime \prime}} & =\sum_{q^{\prime}} K_{j^{\prime} q q^{\prime}}^{j^{\prime \prime}}\left[\phi_{q^{\prime}} \Delta_{j^{\prime \prime}} w\right]
\end{aligned}
$$

where the operator $K_{j^{\prime} q q^{\prime}},\left(\right.$ resp. $\left.K_{j^{\prime} q q^{\prime}}^{j^{\prime \prime}}\right)$ is given by

$$
K_{j^{\prime} q q^{\prime}} w(x)=\phi_{q}(x) c(t, x) 2^{j^{\prime} d} \int h\left(2^{j^{\prime}}(x-y)\right) a(t, y) \widetilde{\phi}_{q^{\prime}}(y) w(y) d y
$$

(resp.

$$
\left.K_{j^{\prime} q q^{\prime}}^{j^{\prime \prime}} w(x)=\phi_{q}(x) c(t, x) 2^{j^{\prime} d} \int h\left(2^{j^{\prime}}(x-y)\right) a(t, y) \widetilde{\Delta}_{j^{\prime \prime}}\left(\widetilde{\phi}_{q^{\prime}}(y) w(y)\right) d y\right)
$$

for $h \in \mathcal{S}\left(\mathbb{R}^{d}\right)$ with $\widehat{h} \in C_{0}^{\infty}\left(\mathbb{R}^{d}\right)$. Let us remark that the kernel $k_{j^{\prime} q q^{\prime}}(x, y)$ of $K_{j^{\prime} q q^{\prime}}$ satisfies for any $N \in \mathbb{N}$

$$
\begin{aligned}
& \sup _{x} \int\left|k_{j^{\prime} q q^{\prime}}(x, y)\right| d y \leqslant C_{N}\left\langle q-q^{\prime}\right\rangle^{-N}, \\
& \sup _{y} \int\left|k_{j^{\prime} q q^{\prime}}(x, y)\right| d x \leqslant C_{N}\left\langle q-q^{\prime}\right\rangle^{-N},
\end{aligned}
$$

since, on the domain $|y| \geqslant \varepsilon|x|(\varepsilon>0)|c(t, x) a(t, y)| \leqslant C$ as $m_{1} \leqslant 0, m_{1}^{\prime} \leqslant 0$, and on the domain $|y|<\varepsilon|x|$, the decay of $h$ compensates the growth of $c(t, x)$, since 
$2^{j^{\prime}} \geqslant c 2^{\ell / 2} \geqslant c \sqrt{t}$ on the support of $\Gamma_{\ell}(t)$. Consequently, for $t \sim 2^{\ell}, \sigma \in[1,+\infty]$

$$
\left\|K_{j^{\prime} q q^{\prime}}\right\|_{\mathcal{L}\left(L^{\sigma}, L^{\sigma}\right)} \leqslant C_{N}\left\langle q-q^{\prime}\right\rangle^{-N}
$$

for any $N$, uniformly in $j^{\prime}, q, q^{\prime}$. By integration by parts, we can write (3.2.76)

$$
\begin{aligned}
\phi_{q}(x) c(t, x) 2^{j^{\prime} d} \int\left(2^{-j^{\prime \prime}} D_{y}\right)^{M}\left[h \left(2^{j^{\prime}}(x-\right.\right. & y)) a(t, y)] \\
& \times\left(2^{-j^{\prime \prime}} D_{y}\right)^{-M} \widetilde{\Delta}_{j^{\prime \prime}}\left[\widetilde{\phi}_{q^{\prime}}(y) w(y)\right] d y
\end{aligned}
$$

for any $M \in \mathbb{N}$. Since, by (3.2.40),

$$
\left|D_{y}^{\gamma} a(t, y)\right| \leqslant C t^{|\gamma| / 2}(1+\sqrt{t}|y|)^{m_{1}}(1+|y|)^{m_{1}^{\prime}}
$$

and, for $t \sim 2^{\ell}, \sqrt{t} \leqslant C 2^{\ell / 2} \leqslant C 2^{j}$, we deduce from (3.2.79) that the kernel $k_{j^{\prime} q q^{\prime}}^{j^{\prime \prime}}(x, y)$ of $K_{j^{\prime} q q^{\prime}}^{j^{\prime \prime}}$ satisfies

$$
\begin{aligned}
& \sup _{x} \int\left|k_{j^{\prime} q q^{\prime}}^{j^{\prime \prime}}(x, y)\right| d y \leqslant C_{N}\left\langle q-q^{\prime}\right\rangle^{-N} 2^{-N\left(j^{\prime \prime}-j^{\prime}\right)}, \\
& \sup _{y} \int\left|k_{j^{\prime} q q^{\prime}}^{j^{\prime \prime}}(x, y)\right| d x \leqslant C_{N}\left\langle q-q^{\prime}\right\rangle^{-N} 2^{-N\left(j^{\prime \prime}-j^{\prime}\right)},
\end{aligned}
$$

whence, for $t \sim 2^{\ell}, \sigma \in[1,+\infty]$

$$
\left\|K_{j^{\prime} q q^{\prime}}^{j^{\prime \prime}}\right\|_{\mathcal{L}\left(L^{\sigma}, L^{\sigma}\right)} \leqslant C_{N}\left\langle q-q^{\prime}\right\rangle^{-N} 2^{-N\left(j^{\prime \prime}-j^{\prime}\right)} .
$$

Let us estimate the first contribution to the right hand side of (3.2.73). Using (3.2.74), (3.2.78) we see that

$$
\begin{aligned}
& \left\|\phi_{q}(x) U_{j^{\prime}}^{\prime} \Gamma_{\ell}(t)\right\|_{L^{p}\left(\frac{d t}{t}, L^{\infty}(d x)\right)}\left\|V_{j^{\prime}} \Gamma_{\ell}(t)\right\|_{L^{\infty}\left(d t, L^{2}(d x)\right)} \\
& \qquad C_{N} \sum_{q^{\prime}}\left\langle q-q^{\prime}\right\rangle^{-N}\left\|\phi_{q^{\prime}}(x) \chi(D / \sqrt{t}) w \Gamma_{\ell}(t)\right\|_{L^{p}\left(\frac{d t}{t}, L^{\infty}(d x)\right)} \\
& \quad \times\left\|V_{j^{\prime}} \Gamma_{\ell}(t)\right\|_{L^{\infty}\left(d t, L^{2}(d x)\right)} .
\end{aligned}
$$

Applying (3.2.54) and (3.2.43) with $a=c^{-1}, \nu=d / 2$ in case (i) (resp. (3.2.59) and (3.2.43) with $a=c^{-1}, \nu=0$ in case (ii)), we get the upper bound

$$
C 2^{-\left(s-m_{1}-m_{2}\right)(j-\ell / 2)} 2^{(j-\ell) \alpha^{\prime}} c_{j q}
$$

in both cases, for a $\ell_{j}^{2} \ell_{q}^{r_{p}}$ sequence $\left(c_{j q}\right)_{j q}$. This is the wanted conclusion. To study the second contribution to the right hand side of (3.2.73) we write using again (3.2.74), $(3.2 .78)$

$$
\begin{aligned}
& \left\|\phi_{q}(x) U_{j^{\prime}}^{\prime \prime} \Gamma_{\ell}(t)\right\|_{L^{p}\left(\frac{d t}{t}, L^{2}(d x)\right)}\left\|V_{j^{\prime}} \Gamma_{\ell}(t)\right\|_{L^{\infty}(d t d x)} \\
& \leqslant C_{N} \sum_{q^{\prime}}\left\langle q-q^{\prime}\right\rangle^{-N}\left\|\phi_{q^{\prime}}(x)(1-\chi)(D / \sqrt{t}) S_{j^{\prime}+N_{2}} w \Gamma_{\ell}(t)\right\|_{L^{p}\left(\frac{d t}{t}, L^{2}(d x)\right)} \\
& \times\left\|V_{j^{\prime}} \Gamma_{\ell}(t)\right\|_{L^{\infty}(d t d x)} .
\end{aligned}
$$


Since (3.2.44) with $a=c^{-1}$ implies in any case $\left\|V_{j^{\prime}} \Gamma_{\ell}(t)\right\|_{L^{\infty}(d t d x)} \leqslant C 2^{(j-\ell / 2)\left(m_{1}+m_{2}\right)}$ we obtain from (3.2.57) the upper bound

$$
C 2^{-\left(s-m_{1}-m_{2}\right)(j-\ell / 2)} 2^{(j-\ell) \alpha^{\prime}} c_{j q}^{\prime}
$$

for a $\ell_{j}^{2} \ell_{q}^{r_{p}}$ sequence $\left(c_{j q}^{\prime}\right)_{j q}$. This is again the wanted conclusion.

To study the third contribution to the right hand side of (3.2.73), we write using $(3.2 .74),(3.2 .81)$

$$
\begin{aligned}
& \sum_{j^{\prime \prime} \geqslant j^{\prime}}\left\|\phi_{q}(x) U_{j^{\prime}}^{j^{\prime \prime}} \Gamma_{\ell}(t)\right\|_{L^{p}\left(\frac{d t}{t}, L^{2}(d x)\right)}\left\|V_{j^{\prime}} \Gamma_{\ell}(t)\right\|_{L^{\infty}(d t d x)} \\
& \leqslant C_{N} \sum_{q^{\prime}} \sum_{j^{\prime \prime} \geqslant j^{\prime}}\left\langle q-q^{\prime}\right\rangle^{-N} 2^{-N\left(j^{\prime \prime}-j^{\prime}\right)}\left\|\phi_{q^{\prime}}(x) \Delta_{j^{\prime \prime}} w \Gamma_{\ell}(t)\right\|_{L^{p}\left(\frac{d t}{t}, L^{2}(d x)\right)} \\
& \times\left\|V_{j^{\prime}} \Gamma_{\ell}(t)\right\|_{L^{\infty}(d t d x)} .
\end{aligned}
$$

Using (3.2.44) and the definition of $E_{\alpha, \alpha^{\prime}, r}^{s, s^{\prime}}$, we estimate (3.2.83) by

$$
\begin{array}{r}
C_{N} \sum_{q^{\prime}} \sum_{j^{\prime \prime} \geqslant j^{\prime}}\left\langle q-q^{\prime}\right\rangle^{-N} 2^{-N\left(j^{\prime \prime}-j^{\prime}\right)} 2^{-\left(j^{\prime \prime}-\ell / 2\right) s} 2^{\left(j^{\prime \prime}-\ell\right) \alpha^{\prime}}\left(1+2^{j^{\prime \prime}-\ell}\right)^{-s_{p}^{\prime}-\alpha^{\prime}} c_{j^{\prime \prime} q^{\prime}} \\
\times 2^{\left(j^{\prime}-\ell / 2\right)\left(m_{1}+m_{2}\right)} \\
\leqslant C 2^{-(j-\ell / 2)\left(s-m_{1}-m_{2}\right)} 2^{(j-\ell) \alpha^{\prime}} c_{j q}^{\prime}
\end{array}
$$

with a $\ell_{j^{\prime \prime}}^{2} \ell_{q^{\prime}}^{r_{p}}\left(\operatorname{resp} . \ell_{j^{2}}^{2} \ell_{q}^{r_{p}}\right)$ sequence $\left(c_{j^{\prime \prime} q^{\prime}}\right)_{j^{\prime \prime} q^{\prime}}\left(\operatorname{resp} .\left(c_{j q}^{\prime}\right)_{j q}\right)$. This gives the wanted conclusion.

Estimate for $\ell \leqslant j$. — Using decomposition (3.2.72), we estimate

$$
\begin{aligned}
\left\|\phi_{q} U_{j^{\prime}} V_{j^{\prime}} \Gamma_{\ell}(t)\right\|_{L^{p}\left(\frac{d t}{t}, L^{2}\right)} \leqslant\left\|\phi_{q} U_{j^{\prime}}^{\prime} \Gamma_{\ell}(t)\right\|_{L^{p}\left(\frac{d t}{t}, L^{2}\right)}\left\|\Gamma_{\ell}(t) V_{j^{\prime}}\right\|_{L^{\infty}(d t d x)} \\
+\left\|\phi_{q} U_{j^{\prime}}^{\prime \prime} \Gamma_{\ell}(t)\right\|_{L^{p}\left(\frac{d t}{t}, L^{2}\right)}\left\|\Gamma_{\ell}(t) V_{j^{\prime}}\right\|_{L^{\infty}(d t d x)} \\
+\sum_{j^{\prime \prime} \geqslant j^{\prime}}\left\|\phi_{q} U_{j^{\prime}}^{j^{\prime \prime}} \Gamma_{\ell}(t)\right\|_{L^{p}\left(\frac{d t}{t}, L^{2}\right)}\left\|\Gamma_{\ell}(t) V_{j^{\prime}}\right\|_{L^{\infty}(d t d x)} .
\end{aligned}
$$

We estimate the first term in the right hand side using (3.2.74), (3.2.78), (3.2.56) and $(3.2 .44)$ by

$$
C 2^{-\left(s-m_{1}-m_{2}\right)(j-\ell / 2)-\left(s_{p}^{\prime}-m_{1}^{\prime}\right)(j-\ell)} c_{j q}
$$

for a $\ell_{j}^{2} \ell_{q}^{r_{p}}$ sequence $\left(c_{j q}\right)_{j q}$. This is the wanted conclusion. The second contribution to the right hand side of (3.2.84) is estimated in the same way, using (3.2.57) instead of (3.2.56). Finally, the third contribution is smaller, by (3.2.81), (3.2.44) and the definition of $E_{\alpha, \alpha^{\prime}, r}^{s, s^{\prime}}$, than

$$
C_{N} \sum_{j^{\prime \prime} \geqslant j^{\prime}} \sum_{q^{\prime}}\left\langle q-q^{\prime}\right\rangle^{-N} 2^{-N\left(j^{\prime \prime}-j^{\prime}\right)} 2^{\left(-j^{\prime \prime}-\ell / 2\right) s-\left(j^{\prime \prime}-\ell\right) s_{p}^{\prime}} c_{j^{\prime \prime} q^{\prime}} 2^{(j-\ell) m_{1}^{\prime}} 2^{(j-\ell / 2)\left(m_{1}+m_{2}\right)}
$$

for a $\ell_{j^{\prime \prime}}^{2} \ell_{q^{\prime}}^{r_{p}}$ sequence $\left(c_{j^{\prime \prime} q^{\prime}}\right)_{j^{\prime \prime} q^{\prime}}$. This is again smaller than $(3.2 .85)$, which concludes the proof of the proposition. 
We close this chapter with a corollary of proposition 3.2.4 which will be used in the rest of this paper. Remind that we defined in definition 3.1.7 spaces $\mathcal{E}_{\alpha, \alpha^{\prime}, r}^{s, s^{\prime}}(M)$, $\widetilde{\mathcal{E}}_{\alpha, \alpha^{\prime}, r}^{s, s^{\prime}}(M)$.

Corollary 3.2.7. - Take $d=2$ and let $b(t, \xi) \in \Sigma_{\nu}^{m_{2}}$. Let $m \in \mathbb{Z}^{*}, M \in 2 \mathbb{N}$. Let $w$ be an element of $\widetilde{\mathcal{E}}_{\alpha, \alpha^{\prime}, r}^{s, s^{\prime}}(M)$ such that for a given $\mu \in[0,4],\langle\sqrt{t} x\rangle^{\mu} w \in \widetilde{\mathcal{E}}_{\alpha, \alpha^{\prime}, r}^{s-\mu, s^{\prime}}(M)$. Assume

(3.2.86) $\quad-2<\alpha+\alpha^{\prime}<-1, \quad s-\mu<\alpha+2 \alpha^{\prime}, \quad s+s^{\prime}-\mu-M<-1 / 2, \quad \nu=1$ or

$$
-1<\alpha+\alpha^{\prime}, \quad s-\mu<\alpha^{\prime}-1, \quad s+s^{\prime}-\mu-M<-1 / 2, \quad \nu=0 .
$$

Then under assumptions (3.2.86), (resp. (3.2.87)) $T_{w} b(t, D)\left(e^{i m \theta}\right)$ belongs to $\mathcal{E}_{\alpha+4, \alpha^{\prime}, r}^{s-m_{2}, s^{\prime}}(M)\left(\operatorname{resp} . \mathcal{E}_{\alpha+2, \alpha^{\prime}, r}^{s-m_{2}, s^{\prime}}(M)\right)$.

Proof. - Take $\gamma \in \mathbb{N}^{d}$ with $|\gamma| \leqslant M$. We have to show that the expression $x^{\gamma} T_{w} b(t, D)\left(e^{i m \theta}\right)$ belongs to $E_{\alpha+4, \alpha^{\prime}, r}^{s-m_{2}, s^{\prime}-|\gamma|}$ (resp. $\left.E_{\alpha+2, \alpha^{\prime}, r}^{s-m_{2}, s^{\prime}-|\gamma|}\right)$. Using the notations introduced in (3.1.16), we can write this expression as a linear combination of quantities $\widetilde{T}_{x \gamma^{\prime} w} b(t, D)\left(e^{i m \theta}\right)$ for $\left|\gamma^{\prime}\right| \leqslant|\gamma|$. Write $x^{\gamma^{\prime}} w=a \widetilde{w}$ with

$$
\begin{aligned}
a(t, x) & =x^{\gamma^{\prime}}\left(1+x^{2}\right)^{-M / 2}\langle\sqrt{t} x\rangle^{-\mu} \\
\widetilde{w} & =\langle\sqrt{t} x\rangle^{\mu}\left(1+x^{2}\right)^{M / 2} w .
\end{aligned}
$$

We see that $a$ satisfies (3.2.40) with $m_{1}=-\mu \leqslant 0, m_{1}^{\prime}=-M+\left|\gamma^{\prime}\right| \leqslant-M+|\gamma| \leqslant 0$. Moreover, by assumption, $\widetilde{w} \in \widetilde{E}_{\alpha, \alpha^{\prime}, r}^{s-\mu, s^{\prime}-M}$. When (3.2.86), (resp. (3.2.87)) is satisfied, we deduce from (i) (resp. from (ii)) of proposition 3.2.4 that $T_{a \widetilde{w}} b(t, D)\left(e^{i m \theta}\right)$ belongs to $E_{\alpha+4, \alpha^{\prime}, r}^{s-m_{2}, s^{\prime}-|\gamma|}$ (resp. $E_{\alpha+2, \alpha^{\prime}, r}^{s-m_{2}, s^{\prime}-|\gamma|}$ ) which is the wanted conclusion. 



\section{CHAPTER 4}

\section{PROOF OF THE MAIN THEOREM}

\subsection{Main reductions}

Let us recall that we have seen in section 1.2 that theorem 1.1.1 will be proved if we succeed to construct a solution to the equation

$$
\begin{aligned}
\left(D_{t}+\frac{D_{x}^{2}}{t^{2}}\right) w & =\frac{1}{t} e^{i \theta(t, x)} Q_{1}(w, Z w)+\frac{1}{t} e^{-3 i \theta(t, x)} Q_{2}(\bar{w}, \overline{Z w}) . \\
\left.w\right|_{t=1} & =\varepsilon w_{0}
\end{aligned}
$$

defined for $t \geqslant 1$, when $\varepsilon$ is small enough and $w_{0}$ stays in the unit ball of $\mathcal{H}^{M+4}\left(\mathbb{R}^{2}\right)$.

Using the definition of $Z=\frac{D_{x}}{t}+\frac{x}{2}$ and the assumptions on $Q_{1}, Q_{2}$, we can rewrite the first equation (4.1.1) as

$$
\left(D_{t}+\frac{D_{x}^{2}}{t^{2}}\right) w=\frac{1}{t} \sum_{|\gamma| \leqslant 2} q_{1}^{\gamma}\left(w, \frac{D_{x}}{t} w\right) x^{\gamma} e^{i \theta}+\frac{1}{t} \sum_{|\gamma| \leqslant 2} q_{2}^{\gamma}\left(\bar{w}, \frac{D_{x}}{t} \bar{w}\right) x^{\gamma} e^{-3 i \theta}
$$

where $\gamma \in \mathbb{N}^{d},|\gamma| \leqslant 2$ in the summation, and $q_{1}^{\gamma}\left(Y_{0}, Y_{1}\right), q_{2}^{\gamma}\left(Y_{0}, Y_{1}\right)$ are homogeneous of degree 2 in $\left(Y_{0}, Y_{1}\right)$, their degree in $Y_{1} \operatorname{deg}_{Y_{1}} q_{j}^{\gamma}\left(Y_{0}, Y_{1}\right)$ satisfying the relation

$$
0<\operatorname{deg}_{Y_{1}} q_{j}^{\gamma}\left(Y_{0}, Y_{1}\right)+|\gamma| \leqslant 2, \quad j=1,2 .
$$

The spaces we will use to look for a solution are the spaces $\widetilde{\mathcal{E}}_{\alpha, \alpha^{\prime}, r}^{s, s^{\prime}}(M)$ and $\underline{\mathcal{E}}_{\alpha, \alpha^{\prime}, r}^{s, s^{\prime}}(M)$ introduced in (3.1.49). Let us recall their definition

$$
\begin{aligned}
& \widetilde{\mathcal{E}}_{\alpha, \alpha^{\prime}, r}^{s, s^{\prime}}(M)=\left\{v \in \widetilde{E}_{\alpha, \alpha^{\prime}, r}^{s, s^{\prime}} ; \forall \gamma \in \mathbb{N}^{d},|\gamma| \leqslant M, x^{\gamma} v \in \widetilde{E}_{\alpha, \alpha^{\prime}, r}^{s, s^{\prime}-|\gamma|}\right\} \\
& \underline{\widetilde{\mathcal{E}}}_{\alpha, \alpha^{\prime}, r}^{s, s^{\prime}}(M)=\left\{v \in \underline{\widetilde{E}}_{\alpha, \alpha^{\prime}, r}^{s, s^{\prime}} ; \forall \gamma \in \mathbb{N}^{d},|\gamma| \leqslant M, x^{\gamma} v \in \underline{\widetilde{E}}_{\alpha, \alpha^{\prime}, r}^{s, s^{\prime}-|\gamma|}\right\} .
\end{aligned}
$$

Since we have relations

$$
\begin{aligned}
& Z^{\gamma}=\sum_{\gamma^{\prime}+\gamma^{\prime \prime} \leqslant \gamma} a_{\gamma^{\prime}, \gamma^{\prime \prime}}\left(D_{x} / t\right)^{\gamma^{\prime}} x^{\gamma^{\prime \prime}} \\
& x^{\gamma}=\sum_{\gamma^{\prime}+\gamma^{\prime \prime} \leqslant \gamma} b_{\gamma^{\prime}, \gamma^{\prime \prime}}\left(D_{x} / t\right)^{\gamma^{\prime}} Z^{\gamma^{\prime \prime}}
\end{aligned}
$$


with coefficients $a_{\gamma^{\prime}, \gamma^{\prime \prime}}, b_{\gamma^{\prime}, \gamma^{\prime \prime}}$ uniformly bounded for $t \geqslant 1$, we see that these spaces are also characterized by

$$
\begin{aligned}
& \widetilde{\mathcal{E}}_{\alpha, \alpha^{\prime}, r}^{s, s^{\prime}}(M)=\left\{v \in \widetilde{E}_{\alpha, \alpha^{\prime}, r}^{s, s^{\prime}} ; \forall \gamma \in \mathbb{N}^{d},|\gamma| \leqslant M, Z^{\gamma} v \in \widetilde{E}_{\alpha, \alpha^{\prime}, r}^{s, s^{\prime}-|\gamma|}\right\} \\
& \widetilde{\mathcal{E}}_{\alpha, s^{\prime}, r}^{s, r}(M)=\left\{v \in \underline{\widetilde{E}}_{\alpha, \alpha^{\prime}, r}^{s, s^{\prime}} ; \forall \gamma \in \mathbb{N}^{d},|\gamma| \leqslant M, Z^{\gamma} v \in \underline{\underline{E}}_{\alpha, \alpha^{\prime}, r}^{s, s^{\prime}-|\gamma|}\right\} .
\end{aligned}
$$

We also have an injection $\widetilde{\mathcal{E}}_{\alpha, \alpha^{\prime}, \infty}^{s, s^{\prime}}(M) \subset \underline{\widetilde{\mathcal{E}}}_{\alpha, \alpha^{\prime}, \infty}^{s, s^{\prime}}(M)$ because of the corresponding property $\widetilde{H}_{\alpha, \alpha^{\prime}, 2}^{s, s^{\prime}} \subset \underline{\widetilde{H}}_{\alpha, \alpha^{\prime}}^{s, s^{\prime}}$.

The indices $s, s^{\prime}, \alpha, \alpha^{\prime}, M$ used in (4.1.4), (4.1.5) will be fixed satisfying the following conditions

$$
\begin{gathered}
\alpha+2 \alpha^{\prime}+4>s>\alpha^{\prime}+2>3, \quad \alpha+\alpha^{\prime}<-1, \quad M=s+s^{\prime}-4 \geqslant 6 \\
\alpha+2 \alpha^{\prime}+4>s>2\left(\alpha+2 \alpha^{\prime}+2\right) .
\end{gathered}
$$

Remark that the conditions concerning $\left(\alpha, \alpha^{\prime}\right)$ read

$$
-2<\alpha+\alpha^{\prime}<-1, \quad \alpha^{\prime}>1, \quad \frac{\alpha}{2}+\alpha^{\prime}<0
$$

i.e. $\left(\alpha, \alpha^{\prime}\right)$ has to stay in the shaded area in the following figure and $s$ must stay in the

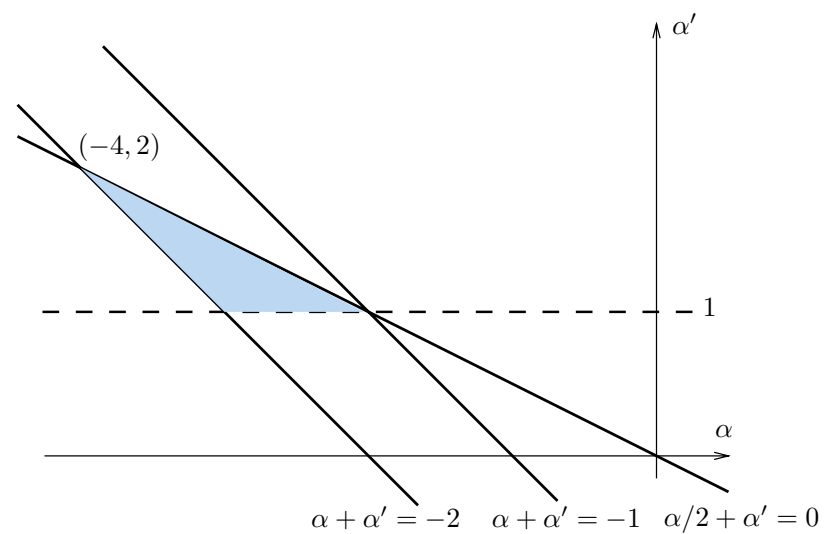

two intervals defined in (4.1.6). One should think of $\left(\alpha, \alpha^{\prime}\right)$ as being close to $(-4,2)$, and in the shaded area, and $s$ being close to $4-0$, in the intervals (4.1.6). The fact that $s$ has to be pretty large is imposed by the nonlinear estimates we will need to study the right hand side of (4.1.2). We cannot hope to put this right hand side in a space $\widetilde{\mathcal{E}}_{\alpha, \alpha^{\prime}, r}^{s, s^{\prime}}(M)$ for such a large $s$ : in fact, this would impose to be able to make act on it $s \frac{D_{x}}{\sqrt{t}}$ derivatives, which is incompatible (if one wants uniform time estimates) with the presence of the exponentials $e^{i \theta}, e^{-3 i \theta}$. To get around this difficulty, the main idea is to get rid of these exponentials exploiting the fact that their phases are non-characteristic for the operator $D_{t}+\frac{D_{x}^{2}}{t^{2}}$. 
We fix now $s, s^{\prime}, \alpha, \alpha^{\prime}, M$ satisfying (4.1.6), we take $\left.\mu \in\right] 0,2[$, close enough to 2 , and introduce the following notations:

$$
\begin{aligned}
& \mathcal{G}=\left\{v \in \widetilde{\mathcal{E}}_{\alpha, \alpha^{\prime}, \infty}^{s, s^{\prime}}(M) ;\left(t D_{t}\right)^{k} v \in \widetilde{\mathcal{E}}_{\alpha, \alpha^{\prime}, \infty}^{s-2 k, s^{\prime}}(M), k=0,1,2\right\} \\
& \mathcal{G}^{\prime}=\left\{f \in \mathcal{E}_{\beta, \alpha^{\prime}, 1}^{s,-1}(M) ; t D_{t} f \in \mathcal{E}_{\beta, \alpha^{\prime}, 1}^{s-2, s^{\prime}-1}(M)\right\} \\
& \mathcal{V}=\left\{V \in \widetilde{\mathcal{E}}_{\beta, \alpha^{\prime}, 1}^{s+2, s^{\prime}-1}(M) ; t D_{t} V \in \widetilde{\mathcal{E}}_{\beta, \alpha^{\prime}, 1}^{s, s^{\prime}-1}(M), \forall c \in S^{\mu}, \forall \gamma \in \mathbb{N}^{2},|\gamma| \leqslant 1\right. \\
&\left.c(\sqrt{t} x)(D / t)^{\gamma}\left(t D_{t}\right)^{k} V \in \widetilde{\mathcal{E}}_{\alpha+2|\gamma|, \alpha^{\prime}, 1}^{s+2-\mu-2 k, s^{\prime}-1-|\gamma|}(M), k=0,1\right\}
\end{aligned}
$$

where the index $\beta$ is defined in terms of $\alpha$ by

$$
\beta=2\left(\alpha+\alpha^{\prime}+1\right)
$$

We will look for the solution $w$ of (4.1.2) as

$$
w=v+V_{1} e^{i \theta}+V_{-3} e^{-3 i \theta}
$$

with $v \in \mathcal{G}, V_{1}, V_{-3} \in \mathcal{V}$. This expression should be understood as the beginning of an expansion of $w$, since the definition of $\mathcal{V}$ in (4.1.8) shows that $V_{1}, V_{-3}$ will decay like $\langle\sqrt{t} x\rangle^{-\mu}$. The two functions $V_{1}, V_{-3}$ will be determined as functions of $v$ in order to cancel the worst oscillating terms in the right hand side of (4.1.2). Let us compute the expression we get when substituting (4.1.10) in the right hand side of (4.1.2). Set the following notations for sets of indices

$$
I_{1}=\{-4,-2,2,0\}, \quad I_{2}=\{-5,-1,3\} .
$$

Denote by $V$ the pair $\left(V_{1}, V_{-3}\right)$. We have the following lemma:

LEMMA 4.1.1. - There are

- quadratic polynomials $P_{\gamma}^{1}\left(Y_{0}, Y_{1}\right), P_{\gamma}^{-3}\left(\bar{Y}_{0}, \bar{Y}_{1}\right)$ in two indeterminates, indexed by $\gamma \in \mathbb{N}^{2}$, satisfying

$$
0<\operatorname{deg}_{Y_{1}} P_{\gamma}^{1}+|\gamma| \leqslant 2,0<\operatorname{deg}_{\bar{Y}_{1}} P_{\gamma}^{-3}+|\gamma| \leqslant 2
$$

- quadratic polynomials $P_{\gamma}^{m}\left(Y_{0}, W_{0}, \bar{Y}_{0}, \bar{W}_{0} ; Y_{1}, W_{1}, \bar{Y}_{1}, \bar{W}_{1}\right)$ in indeterminates $Y_{0}, \bar{Y}_{0} \in \mathbb{C}, Y_{1}, \bar{Y}_{1}, W_{0}, \bar{W}_{0} \in \mathbb{C}^{2}, W_{1}, \bar{W}_{1} \in \mathbb{C}^{4}$, linear in the variables $\left(Y_{0}, \bar{Y}_{0}, Y_{1}, \bar{Y}_{1}\right)$ and $\left(W_{0}, \bar{W}_{0}, W_{1}, \bar{W}_{1}\right)$, indexed by $m \in I_{1}$ and $\gamma \in \mathbb{N}^{2}$, satisfying

$$
0<\operatorname{deg}_{\left(Y_{1}, W_{1}, \bar{Y}_{1}, \bar{W}_{1}\right)} P_{\gamma}^{m}+|\gamma| \leqslant 2,
$$

- quadratic polynomials $P_{\gamma}^{m}\left(W_{0}, \bar{W}_{0} ; W_{1}, \bar{W}_{1}\right)$ indexed by $m \in I_{2}$ and $\gamma \in \mathbb{N}^{2}$ satisfying

$$
0<\operatorname{deg}_{\left(W_{1}, \bar{W}_{1}\right)} P_{\gamma}^{m}+|\gamma| \leqslant 2
$$


such that, if $w$ is given by (4.1.10) we have

$$
\begin{aligned}
\sum_{|\gamma| \leqslant 2} q_{1}^{\gamma}\left(w, \frac{D}{t} w\right) x^{\gamma} e^{i \theta}+\sum_{|\gamma| \leqslant 2} q_{2}^{\gamma}\left(\bar{w}, \frac{D}{t} \bar{w}\right) x^{\gamma} e^{-3 i \theta}=S_{1}+S_{2}+S_{3} \\
S_{1}=\sum_{|\gamma| \leqslant 2} P_{\gamma}^{1}\left(v, \frac{D}{t} v\right) x^{\gamma} e^{i \theta}+\sum_{|\gamma| \leqslant 2} P_{\gamma}^{-3}\left(\bar{v}, \frac{D}{t} \bar{v}\right) x^{\gamma} e^{-3 i \theta} \\
S_{2}=\sum_{m \in I_{1}} \sum_{|\gamma| \leqslant 2} P_{\gamma}^{m}\left(v, V, \bar{v}, \bar{V} ; \frac{D}{t} v, \frac{D}{t} V, \frac{D}{t} \bar{v}, \frac{D}{t} \bar{V}\right) x^{\gamma} e^{i m \theta} \\
S_{3}=\sum_{m \in I_{2}} \sum_{|\gamma| \leqslant 2} P_{\gamma}^{m}\left(V, \bar{V} ; \frac{D}{t} V, \frac{D}{t} \bar{V}\right) x^{\gamma} e^{i m \theta} .
\end{aligned}
$$

The lemma is proved substituting (4.1.10) into the left hand side of (4.1.15) and sorting terms according to the degree of homogeneity in $\left(v, \bar{v}, \frac{D}{t} v, \frac{D}{t} \bar{v}\right)$. Our first task will be to write $S_{1}, S_{2}, S_{3}$ in (4.1.15) making appear the main contributions and remainders. We have to introduce some notations.

Definition 4.1.2. — A quadratic remainder will be a continuous function $v \mapsto R(v)$ (resp. $(v, V) \mapsto R(v, V)$, resp. $V \mapsto R(V))$ defined on the open unit ball of $\mathcal{G}$ (resp. $\mathcal{G} \times \mathcal{V}$, resp. $\mathcal{V})$, with values in $\mathcal{G}^{\prime}$, vanishing at the origin, and such that one has the following estimates for $v, v^{\prime}$ in the unit ball of $\mathcal{G}$ (resp. $(v, V),\left(v^{\prime}, V^{\prime}\right)$ in the unit ball of $\mathcal{G} \times \mathcal{V}$, resp. $V, V^{\prime}$ in the unit ball of $\left.\mathcal{V}\right)$

$$
\begin{gathered}
\left\|R(v)-R\left(v^{\prime}\right)\right\|_{\mathcal{G}^{\prime}} \leqslant C\left(\|v\|_{\mathcal{G}}+\left\|v^{\prime}\right\|_{\mathcal{G}}\right)\left\|v-v^{\prime}\right\|_{\mathcal{G}} \\
\left\|R(v, V)-R\left(v^{\prime}, V^{\prime}\right)\right\|_{\mathcal{G}^{\prime}} \leqslant C\left(\|(v, V)\|_{\mathcal{G} \times \mathcal{V}}+\left\|\left(v^{\prime}, V^{\prime}\right)\right\|_{\mathcal{G} \times \mathcal{V}}\right) \\
\times\left\|\left(v-v^{\prime}, V-V^{\prime}\right)\right\|_{\mathcal{G} \times \mathcal{V}} \\
\left\|R(V)-R\left(V^{\prime}\right)\right\|_{\mathcal{G}^{\prime}} \leqslant C\left(\|V\|_{\mathcal{V}}+\left\|V^{\prime}\right\|_{\mathcal{V}}\right)\left\|V-V^{\prime}\right\|_{\mathcal{V}}
\end{gathered}
$$

(Actually $\mathcal{V}$ is not endowed with a norm, but with a family of semi-norms. We should thus speak not of the unit ball of $\mathcal{V}$, but of a bounded set, and not use a notation like $\|\cdot\| \mathcal{V}$. Anyway, to avoid introducing some more notations, we shall use such an abuse of notations).

Remind that we defined in definition 3.2.1 the class $\Sigma_{\nu}^{\mu}$ of symbols $b(t, \xi)$. Let us denote by $\mathcal{E}, \mathcal{E}^{\prime}, \mathcal{E}^{\prime \prime}$ three generic spaces. We set the following definition:

Definition 4.1.3. - A quadratic symbol $A(v, t, \xi)$ with values in $\mathcal{E} \otimes \Sigma_{\nu}^{\mu}$, such that $t D_{t} A \in \mathcal{E}^{\prime} \otimes \Sigma_{\nu}^{\mu}$, and $\left(t D_{t}\right)^{2} A \in \mathcal{E}^{\prime \prime} \otimes \Sigma_{\nu}^{\mu}$, will be a linear combination of functions $v \mapsto a(v) b(t, \xi)$ with $b \in \Sigma_{\nu}^{\mu}$ and where $a$ is a function defined on the unit ball of $\mathcal{G}$, with values in $\mathcal{E}$, vanishing identically at $v=0$, and such that

$$
\begin{array}{r}
\left\|a(v)-a\left(v^{\prime}\right)\right\|_{\mathcal{E}} \leqslant C\left(\|v\|_{\mathcal{G}}+\left\|v^{\prime}\right\|_{\mathcal{G}}\right)\left\|v-v^{\prime}\right\|_{\mathcal{G}} \\
\left\|t D_{t}\left(a(v)-a\left(v^{\prime}\right)\right)\right\|_{\mathcal{E}^{\prime}} \leqslant C\left(\|v\|_{\mathcal{G}}+\left\|v^{\prime}\right\|_{\mathcal{G}}\right)\left\|v-v^{\prime}\right\|_{\mathcal{G}} \\
\left\|\left(t D_{t}\right)^{2}\left(a(v)-a\left(v^{\prime}\right)\right)\right\|_{\mathcal{E}^{\prime \prime}} \leqslant C\left(\|v\|_{\mathcal{G}}+\left\|v^{\prime}\right\|_{\mathcal{G}}\right)\left\|v-v^{\prime}\right\|_{\mathcal{G}}
\end{array}
$$


for any $v, v^{\prime}$ in the unit ball of $\mathcal{G}$.

We use a similar terminology for symbols $A(v, V, t, \xi)$ defined on the unit ball of $\mathcal{G} \times \mathcal{V}$.

Before beginning the study of equation (4.1.2) let us introduce a last notation. If $A=a \otimes b$ with $a$ a function of $(t, x)$ belonging to a space $\mathcal{E}$ and $b$ a symbol in $\Sigma_{\nu}^{\mu}$, we shall denote by $\operatorname{Op}^{\mathcal{B}}(A)$ the paradifferential operator of symbol $A$, defined using (3.1.12) by

$$
\mathrm{Op}^{\mathcal{B}}(A) u=T_{a(t, x)}(b(t, D) u) .
$$

We shall also use the generic notation $\widetilde{\mathrm{Op}}^{\mathcal{B}}(A)$ for the operator

$$
\widetilde{\mathrm{Op}}^{\mathcal{B}}(A) u=\widetilde{T}_{a(t, x)}(b(t, D) u) .
$$

The study of equation (4.1.2) will be done in several steps. For each of them, we will present the underlying idea on the example used in the introduction, namely the equation

$$
\left(D_{t}+\frac{D_{x}^{2}}{t^{2}}\right) w=\frac{1}{t} e^{i \theta} w\left(\frac{D_{x}}{t} w\right)
$$

where $D_{x}$ denotes generically one of the derivatives $D_{x_{j}}$. The right hand side in the above equation is one of the contributions to the right hand side of (4.1.2).

\section{Step 1: Expression of the equation in terms of $v, V$}

Let us indicate the idea of this first step, when $w$ solves (4.1.20), and when we look for it as $w=v+V_{1} e^{i \theta}$ with some $V_{1}$ to be determined in function of $v$ in a foregoing step. The assumptions $v \in \mathcal{G}, V_{1} \in \mathcal{V}$ mean essentially that $v, V_{1}$ are smooth, and moreover that $V_{1}$ decays essentially like $\langle\sqrt{t} x\rangle^{-2}$. We plug the expression for $w$ in (4.1.20) and get

$$
\begin{aligned}
\left(D_{t}+\frac{D_{x}^{2}}{t^{2}}\right) v= & \frac{1}{t} e^{i \theta} v \frac{D_{x}}{t} v+\frac{1}{t} e^{2 i \theta}\left[v \frac{D_{x}}{t} V_{1}+V_{1} \frac{D_{x}}{t} v\right] \\
& +\frac{1}{t} e^{3 i \theta} V_{1} \frac{D_{x}}{t} V_{1}-\left(D_{t}+\frac{D_{x}^{2}}{t^{2}}\right)\left(V_{1}(v) e^{i \theta}\right) \\
& + \text { other terms. }
\end{aligned}
$$

We look at the different terms in the right hand side of this equation and explain how they appear in (4.1.26) - which is the expression we shall obtain in general. First of all write

$$
V_{1} \frac{D_{x}}{t} V_{1} e^{i \theta}=T_{e^{i \theta}}\left(V_{1} \frac{D_{x}}{t} V_{1}\right)+R\left(e^{i \theta}, V_{1} \frac{D_{x}}{t} V_{1}\right)+T_{V_{1} \frac{D_{x}}{t} V_{1}} e^{i \theta} .
$$

Since $V_{1}$ is smooth, so is $V_{1} \frac{D_{x}}{t} V_{1}$ (with a loss of one $D_{x} / t$-derivative). Consequently, the first two terms in the right hand side of (4.1.22) are smooth, i.e. will contribute to the $R$-term in (4.1.26). The third term in the right hand side of (4.1.22) is also 
smooth: this will be the contains of lemma 4.1.9. Actually, modulo remainders, it can be written

$$
T_{\left(\langle\sqrt{t} x\rangle^{2} V_{1}\right)\left(\langle\sqrt{t} x\rangle^{2} D_{x} V_{1} / t\right)}\left(\langle\sqrt{t} x\rangle^{-4} e^{i \theta}\right) .
$$

Since $\langle\sqrt{t} x\rangle^{-4} e^{i \theta}$ accepts four $D_{x} / \sqrt{t}$-derivatives, and since $V_{1}$ decays essentially like $\langle\sqrt{t} x\rangle^{-2}$, this expression will belong to $\mathcal{G}^{\prime}$ (this uses the fact that the index $s$ satisfies $s<4)$. Consequently we get again a $R$-type contribution to (4.1.26).

Let us look now at

$$
v \frac{D_{x}}{t} v e^{i \theta}=T_{e^{i \theta}}\left(v \frac{D_{x}}{t} v\right)+R\left(e^{i \theta}, v \frac{D_{x}}{t} v\right)+T_{v \frac{D_{x}}{t} v} e^{i \theta} .
$$

The first two terms in the right hand side can be handled as above, and give a $R$-contribution to (4.1.26). The last term $T_{v} \frac{D_{x}}{t} v e^{i \theta}$ has no chance at all of being smooth, as $v$ has no decay (unlike $V_{1}$ ). We thus keep this term in the right hand side of (4.1.26), writing it as $\mathrm{Op}^{\mathcal{B}}\left(A_{1}(v)\right) e^{i \theta}$ where $A_{1}(v)=v \frac{D_{x}}{t} v$ is a symbol independent of $\xi$. Finally, arguing in the same way, we decompose the $e^{2 i \theta}$-term in (4.1.21) as a term of type $\mathrm{Op}^{\mathcal{B}}\left(A_{2}(v, V)\right) e^{2 i \theta}$ and a $R(v, V)$-like term.

Let us turn now to the general case. Our main goal in this first step will be the proof of the following proposition.

Proposition 4.1.4. - Set $M^{\prime}=2 M-5$. There are

- a real number $\mu \in] 0,2[$,

- quadratic symbols $A_{m}(v, t, \xi), m=1,-3$, with values in the space

$$
\widetilde{\mathcal{E}}_{\beta, \alpha^{\prime}, 1}^{s, s^{\prime}-1}\left(M^{\prime}\right) \otimes \Sigma_{0}^{0}+\widetilde{\mathcal{E}}_{\alpha, \alpha^{\prime}, 1}^{s, s^{\prime}-1}\left(M^{\prime}\right) \otimes \Sigma_{1}^{0},
$$

such that, for $k=1,2,\left(t D_{t}\right)^{k} A_{m}(v, t, \xi)$ belongs to

$$
\widetilde{\mathcal{E}}_{\beta, \alpha^{\prime}, 1}^{s-2 k, s^{\prime}-1}\left(M^{\prime}\right) \otimes \Sigma_{0}^{0}+\widetilde{\mathcal{E}}_{\alpha, \alpha^{\prime}, 1}^{s-2 k, s^{\prime}-1}\left(M^{\prime}\right) \otimes \Sigma_{1}^{0},
$$

- quadratic symbols $A_{m}(v, V, t, \xi), m \in I_{1}-\{0\}$, with values in the space $\widetilde{\mathcal{E}}_{\beta, \alpha^{\prime}, 1}^{s, s^{\prime}-1}\left(M^{\prime}\right) \otimes \Sigma_{0}^{0}+\widetilde{\mathcal{E}}_{\alpha, \alpha^{\prime}, 1}^{s, s^{\prime}-1}\left(M^{\prime}\right) \otimes \Sigma_{1}^{0}$, such that for any $c \in S^{\mu^{\prime}}, \mu^{\prime} \in[0, \mu]$ and $k=0,1$

$$
c(\sqrt{t} x)\left(t D_{t}\right)^{k} A_{m}(v, V, t, \xi) \in \widetilde{\mathcal{E}}_{\beta, \alpha^{\prime}, 1}^{s-2 k-\mu^{\prime}, s^{\prime}-1}\left(M^{\prime}\right) \otimes \Sigma_{0}^{0}+\widetilde{\mathcal{E}}_{\alpha, \alpha^{\prime}, 1}^{s-2 k-\mu^{\prime}, s^{\prime}-1}\left(M^{\prime}\right) \otimes \Sigma_{1}^{0},
$$

- two bilinear forms $(v, V) \mapsto H_{j}(v, V), j=1,2$, defined on $\mathcal{G} \times \mathcal{V}$ with values in $\widetilde{\mathcal{E}}_{\alpha, \alpha^{\prime}, 1}^{s, s^{\prime}}(M+1)$, satisfying, for $k=0,1, c \in S^{\mu^{\prime}}, \mu^{\prime} \in[0, \mu]$

$$
\left\|c(\sqrt{t} x)\left(t D_{t}\right)^{k} H_{j}(v, V)\right\|_{\widetilde{\mathcal{E}}_{\alpha, \alpha^{\prime}, 1}^{s-\mu^{\prime}-2 k, s^{\prime}}(M+1)} \leqslant C\|v\|_{\mathcal{G}}\|V\|_{\mathcal{V}}
$$

- quadratic remainders $v \mapsto R(v),(v, V) \mapsto R(v, V)$ defined on the unit ball of $\mathcal{G}$ and $\mathcal{G} \times \mathcal{V}$ respectively, satisfying the conditions of definition 4.1.2, 
such that if $(v, V)$ satisfies the equation

$$
\begin{aligned}
\left(D_{t}+\frac{D_{x}^{2}}{t^{2}}\right) v=\frac{1}{t} \sum_{m \in\{1,-3\}}\left[\mathrm{Op}^{\mathcal{B}}\left(A_{m}(v, t, \xi)\right)\left(e^{i m \theta}\right)-\left(D_{t}+\frac{D_{x}^{2}}{t^{2}}\right)\left(V_{m} e^{i m \theta}\right)\right] \\
+\frac{1}{t} \sum_{m \in I_{1}-\{0\}} \operatorname{Op}^{\mathcal{B}}\left(A_{m}(v, V, t, \xi)\right)\left(e^{i m \theta}\right) \\
+\frac{1}{t} \sum_{j=1}^{2} x_{j} H_{j}(v, V)+\frac{1}{t} R(v)+\frac{1}{t} R(v, V),
\end{aligned}
$$

then $w$ given by (4.1.10) is a solution to (4.1.2).

The proof will be made of several lemmas showing that $S_{1}, S_{2}, S_{3}$ in (4.1.15) can be reduced to terms in the right hand side of (4.1.26).

Lemma 4.1.5. - We fix $\lambda>2$ close to 2 . Let $\gamma_{1}, \gamma_{2}, \gamma^{\prime} \in \mathbb{N}^{2}$ with $\left|\gamma_{1}\right| \leqslant 1,\left|\gamma_{2}\right| \leqslant 1$, $\left|\gamma^{\prime}\right| \leqslant 2 M-3$.

(i) Let $v_{1}, v_{2}$ be two elements of $\mathcal{G}$. Then for $k=0,1,2$

$$
\left(t D_{t}\right)^{k}\left[x^{\gamma^{\prime}}\left((D / t)^{\gamma_{1}} v_{1}\right)\left((D / t)^{\gamma_{2}} v_{2}\right)\right]
$$

belongs to the space $\widetilde{E}_{\beta, \alpha^{\prime}, 1}^{s-2 k, s^{\prime}-\left(\lambda+\left|\gamma^{\prime}\right|-s^{\prime}+2+\delta\right)_{+}-1}$ when $\left|\gamma_{1}\right|+\left|\gamma_{2}\right|>0$, and to the space $\widetilde{E}_{\alpha, \alpha^{\prime}, 1}^{s-2 k, s^{\prime}-\left(\lambda+\left|\gamma^{\prime}\right|-s^{\prime}+2+\delta\right)_{+}}$when $\gamma_{1}=\gamma_{2}=0$ for any $\delta>0$. Moreover the norm of (4.1.27) in these spaces is estimated by $C\left\|v_{1}\right\|_{\mathcal{G}}\left\|v_{2}\right\|_{\mathcal{G}}$.

(ii) Let $v_{1} \in \mathcal{G}, v_{2} \in \mathcal{V}$. Then for $k=0,1, c \in S^{\mu^{\prime}}, \mu^{\prime} \in[0, \mu]$

$$
c(\sqrt{t} x)\left(t D_{t}\right)^{k}\left[x^{\gamma^{\prime}}\left((D / t)^{\gamma_{1}} v_{1}\right)\left((D / t)^{\gamma_{2}} v_{2}\right)\right]
$$

belongs to the space $\widetilde{E}_{\beta, \alpha^{\prime}, 1}^{s-2 k-\mu^{\prime}, s^{\prime}-\left(\lambda+\left|\gamma^{\prime}\right|-s^{\prime}+2+\delta\right)_{+}-1}$ when $\left|\gamma_{1}\right|+\left|\gamma_{2}\right|>0$ and to the space $\widetilde{E}_{\alpha, \alpha^{\prime}, 1}^{s-2 k-\mu^{\prime}, s^{\prime}-\left(\lambda+\left|\gamma^{\prime}\right|-s^{\prime}+2+\delta\right)_{+}}$when $\gamma_{1}=\gamma_{2}=0$ for any $\delta>0$. Moreover the norm of (4.1.28) in these spaces is estimated by $C\left\|v_{1}\right\|_{\mathcal{G}}\left\|v_{2}\right\|_{\mathcal{V}}$.

(iii) Let $v_{1} \in \mathcal{V}, v_{2} \in \mathcal{V}$. Then for $k=0,1, c \in S^{\mu^{\prime}}, \mu^{\prime} \in[0, \mu]$

$$
c(\sqrt{t} x)\left(t D_{t}\right)^{k}\left[x^{\gamma^{\prime}}\left((D / t)^{\gamma_{1}} v_{1}\right)\left((D / t)^{\gamma_{2}} v_{2}\right)\right]
$$

belongs to the space $\widetilde{E}_{\beta, \alpha^{\prime}, 1}^{s-2 k-\mu^{\prime}, s^{\prime}-\left(\lambda+\left|\gamma^{\prime}\right|-s^{\prime}+2+\delta\right)_{+}-1}$ when $\left|\gamma_{1}\right|+\left|\gamma_{2}\right|>0$ and to the space $\widetilde{E}_{\alpha, \alpha^{\prime}, 1}^{s-2 k-\mu^{\prime}, s^{\prime}-\left(\lambda+\left|\gamma^{\prime}\right|-s^{\prime}+2+\delta\right)_{+}}$when $\gamma_{1}=\gamma_{2}=0$, and for $c \in S^{2 \mu^{\prime}}, \mu^{\prime} \in[0, \mu]$

$$
c(\sqrt{t} x)\left[x^{\gamma^{\prime}}\left((D / t)^{\gamma_{1}} v_{1}\right)\left((D / t)^{\gamma_{2}} v_{2}\right)\right]
$$

belongs to the space $\widetilde{E}_{\beta, \alpha^{\prime}, 1}^{s-2 \mu^{\prime} s^{\prime}-\left(\lambda+\left|\gamma^{\prime}\right|-s^{\prime}+2+\delta\right)_{+-1}^{-1}}$ when $\left|\gamma_{1}\right|+\left|\gamma_{2}\right|>0$ and to the space $\widetilde{E}_{\alpha, \alpha^{\prime}, 1}^{s-2 \mu^{\prime}, s^{\prime}-\left(\lambda+\left|\gamma^{\prime}\right|-s^{\prime}+2+\delta\right)_{+}}$when $\gamma_{1}=\gamma_{2}=0$. Moreover the norm of (4.1.28) in these spaces is estimated by $C\left\|v_{1}\right\| \mathcal{V}\left\|v_{2}\right\|_{\mathcal{V}}$. 
Proof

(i) The quantity to study may be written as a combination of expressions $x^{\gamma^{\prime}} w_{1} w_{2}$ with

$$
\begin{aligned}
& w_{1}=\left(t D_{t}\right)^{k_{1}}\left(\frac{D}{t}\right)^{\gamma_{1}} v_{1} \in \widetilde{\mathcal{E}}_{\alpha, \alpha^{\prime}+\left|\gamma_{1}\right|, \infty}^{s-2 k_{1}, s^{\prime}-\left|\gamma_{1}\right|}(M) \subset \widetilde{\mathcal{E}}_{\alpha+2\left|\gamma_{1}\right|, \alpha^{\prime}, \infty}^{s-2 k_{1}, s^{\prime}-\left|\gamma_{1}\right|}(M) \\
& w_{2}=\left(t D_{t}\right)^{k_{2}}\left(\frac{D}{t}\right)^{\gamma_{2}} v_{2} \in \underline{\widetilde{\mathcal{E}}}_{\alpha, \alpha^{\prime}+\left|\gamma_{2}\right|, \infty}^{s-2 k_{2}, s^{\prime}-\left|\gamma_{2}\right|}(M) \subset \underline{\widetilde{\mathcal{E}}}_{\alpha+2\left|\gamma_{2}\right|, \alpha^{\prime}, \infty}^{s-2 k_{2}, s^{\prime}-\left|\gamma_{2}\right|}(M)
\end{aligned}
$$

for $k_{1}+k_{2}=k$. When $\left|\gamma_{1}\right|+\left|\gamma_{2}\right|>0$, one of the first lower indices of the above spaces equals $\alpha+2$, so we can apply (i) of corollary 3.1 .8 with $\sigma_{j}=2 k_{j}, \sigma_{j}^{\prime}=\left|\gamma_{j}\right|$. One checks that assumptions (3.1.50), (3.1.51) are satisfied because of (4.1.6) and of $\lambda>2$. Moreover, condition (3.1.52) is satisfied as long as

$$
s+s^{\prime}>2+\frac{1}{2}\left(\left|\gamma^{\prime}\right|+\left|\gamma_{1}\right|+\left|\gamma_{2}\right|\right)+1
$$

(since $\sigma_{1}+\sigma_{2} \leqslant 4$ ), which is true since $s+s^{\prime}=M+4,\left|\gamma^{\prime}\right| \leqslant 2 M-3$. By (3.1.53) we get that $x^{\gamma^{\prime}} w_{1} w_{2}$ is in the wanted space. When $\gamma_{1}=\gamma_{2}=0$, we use (ii) of corollary 3.1.8, and get also the conclusion of the lemma, since in this case $\max \left(\sigma_{1}^{\prime}, \sigma_{2}^{\prime}\right)=0$.

(ii) We write expression (4.1.28) in terms of $x^{\gamma^{\prime}} w_{1} w_{2}$ with

$$
\begin{aligned}
& w_{1}=\left(t D_{t}\right)^{k_{1}}\left(\frac{D}{t}\right)^{\gamma_{1}} v_{1} \in \underline{\widetilde{\mathcal{E}}}_{\alpha, \alpha^{\prime}+\left|\gamma_{1}\right|, \infty}^{s-2 k_{1}, s^{\prime}-\left|\gamma_{1}\right|}(M) \subset \underline{\widetilde{\mathcal{E}}}_{\alpha+2\left|\gamma_{1}\right|, \alpha^{\prime}, \infty}^{s-2 k_{1}, s^{\prime}-\left|\gamma_{1}\right|}(M) \\
& w_{2}=c(\sqrt{t} x)\left(t D_{t}\right)^{k_{2}}\left(\frac{D}{t}\right)^{\gamma_{2}} v_{2} \in \widetilde{\mathcal{E}}_{\alpha+2\left|\gamma_{2}\right|, \alpha^{\prime}, \infty}^{s-\mu^{\prime}-2 k_{2}, s^{\prime}-\left|\gamma_{2}\right|}(M) \subset \underline{\mathcal{E}}_{\alpha+2\left|\gamma_{2}\right|, \alpha^{\prime}, \infty}^{s-\mu^{\prime}-2 k_{2}, s^{\prime}-\left|\gamma_{2}\right|}(M)
\end{aligned}
$$

for $k_{1}+k_{2}=k$, where the last inclusion in the first line comes from the definition of the spaces. We then apply corollary 3.1 .8 as in the proof of (i), taking here $\sigma_{1}=2 k_{1}$, $\sigma_{2}=2 k_{2}+\mu^{\prime}$ which still satisfies $\sigma_{1}+\sigma_{2} \leqslant 4$ since $k \leqslant 1, \mu^{\prime} \leqslant 2$.

(iii) In this case, (4.1.29) is estimated as (4.1.28). For (4.1.30), we write this expression in terms of $x^{\gamma^{\prime}} w_{1} w_{2}$ where, if $c_{j} \in S^{\mu^{\prime}}$ with $c_{1} c_{2}=c$,

$$
w_{j}=c_{j}(\sqrt{t} x)\left(\frac{D}{t}\right)^{\gamma_{j}} v_{j} \in \widetilde{\mathcal{E}}_{\alpha+2\left|\gamma_{j}\right|, \alpha^{\prime}, \infty}^{s-\mu^{\prime}, s^{\prime}-\left|\gamma_{j}\right|}(M)
$$

and we apply again corollary 3.1.8.

Let us study now term $S_{1}$ of (4.1.15).

LEMMA 4.1.6. - Set $M^{\prime}=2 M-5$. There are quadratic symbols $A_{m}(v, t, \xi), m=$ $1,-3$, with values in $\widetilde{\mathcal{E}}_{\beta, \alpha^{\prime}, 1}^{s, s^{\prime}-1}\left(M^{\prime}\right) \otimes \Sigma_{0}^{0}+\widetilde{\mathcal{E}}_{\alpha, \alpha^{\prime}, 1}^{s, s^{\prime}-1}\left(M^{\prime}\right) \otimes \Sigma_{1}^{0}$ such that for $k=1,2$, $\left(t D_{t}\right)^{k} A_{m} \in \widetilde{\mathcal{E}}_{\beta, \alpha^{\prime}, 1}^{s-2 k, s^{\prime}-1}\left(M^{\prime}\right) \otimes \Sigma_{0}^{0}+\widetilde{\mathcal{E}}_{\alpha, \alpha^{\prime}, 1}^{s-2 k, s^{\prime}-1}\left(M^{\prime}\right) \otimes \Sigma_{1}^{0}$, and a quadratic remainder $v \mapsto R(v)$ such that

$$
S_{1}=\sum_{m \in\{1,-3\}} \operatorname{Op}^{\mathcal{B}}\left(A_{m}(v, t, \xi)\right)\left(e^{i m \theta}\right)+R(v) .
$$

Proof. - We will study the case of contribution $P_{\gamma}^{1}(v,(D / t) v) e^{i \theta} x^{\gamma}$ to $S_{1}$, the other contribution being treated in the same way. Because of (4.1.12) such an expression is a linear combination of quantities of type $\left((D / t)^{\gamma_{1}} v\right)\left((D / t)^{\gamma_{2}} v\right) x^{\gamma_{3}} e^{i \theta}$ for $\gamma_{1}, \gamma_{2}, \gamma_{3}$ 
satisfying $\left|\gamma_{1}\right| \leqslant 1,\left|\gamma_{2}\right| \leqslant 1,0<\left|\gamma_{1}\right|+\left|\gamma_{2}\right|+\left|\gamma_{3}\right| \leqslant 2$. We decompose such an expression as

$$
\begin{aligned}
\mathrm{Op}^{\mathcal{B}}\left(x^{\gamma_{3}} e^{i \theta}\right)\left[\left((D / t)^{\gamma_{1}} v\right)\left((D / t)^{\gamma_{2}} v\right)\right]+R\left(x^{\gamma_{3}} e^{i \theta},\left((D / t)^{\gamma_{1}} v\right)\left((D / t)^{\gamma_{2}} v\right)\right) \\
+\mathrm{Op}^{\mathcal{B}}\left((D / t)^{\gamma_{1}} v(D / t)^{\gamma_{2}} v\right)\left(x^{\gamma_{3}} e^{i \theta}\right)=W_{1}+W_{2}+W_{3} .
\end{aligned}
$$

To show that $W_{1}$ is a quadratic remainder, we just have to prove by definition of $\mathcal{G}^{\prime}$ that for any $\gamma \in \mathbb{N}^{2}$ with $|\gamma| \leqslant M$, any $k=0,1$, any $v_{1}, v_{2} \in \mathcal{G}$,

$$
\left(t D_{t}\right)^{k} x^{\gamma} \mathrm{Op}^{\mathcal{B}}\left(x^{\gamma_{3}} e^{i \theta}\right)\left[\left((D / t)^{\gamma_{1}} v_{1}\right)\left((D / t)^{\gamma_{2}} v_{2}\right)\right]
$$

belongs to $E_{\beta, \alpha^{\prime}, 1}^{s-2 k, s^{\prime}-|\gamma|-1}$, and that its norm in this space is estimated by $C\left\|v_{1}\right\| \mathcal{G}\left\|v_{2}\right\|_{\mathcal{G}}$. Remark that it follows from formula (3.1.12) that when we multiply a paraproduct $T_{a} b$ by $x, x T_{a} b$ may be written, commuting $x$ with the $\Delta_{j}$ 's, as a linear combination of terms of type $\widetilde{T}_{a} b, T_{a}(x b)$. In the same way, an expression $T_{(x a)} b$ is a linear combination of expressions $\widetilde{T}_{a} b, T_{a}(x b)$. Applying this to (4.1.33), we see that this term may be written as a linear combination of expressions

$$
\mathrm{Op}^{\mathcal{B}}\left(x^{\gamma_{3}^{\prime}}\left(t D_{t}\right)^{k^{\prime}}\left(e^{i \theta}\right)\right)\left[\left(t D_{t}\right)^{k^{\prime \prime}} x^{\gamma^{\prime}}\left((D / t)^{\gamma_{1}} v_{1}\right)\left((D / t)^{\gamma_{2}} v_{2}\right)\right]
$$

with $k^{\prime}+k^{\prime \prime}=k,\left|\gamma^{\prime}\right| \leqslant|\gamma|+1 \leqslant M+1 \leqslant 2 M-3, \gamma_{3}^{\prime}=0$ when $\left|\gamma_{1}\right|+\left|\gamma_{2}\right|>0,\left|\gamma_{3}^{\prime}\right|=1$ when $\left(\gamma_{1}, \gamma_{2}\right)=(0,0)$. We apply (4.1.27) to the term inside the bracket of (4.1.34). Since $s^{\prime}=M+4-s>5$ as $M>5$, assertion (i) of lemma 4.1.5 shows that this term belongs to $E_{\beta, \alpha^{\prime}, 1}^{s-2 k^{\prime \prime}, s^{\prime}-|\gamma|-1}$ when $\left|\gamma_{1}\right|+\left|\gamma_{2}\right|>0$, and to $E_{\alpha, \alpha^{\prime}, 1}^{s-2 k^{\prime \prime}, s^{\prime}-|\gamma|}$ when $\left(\gamma_{1}, \gamma_{2}\right)=(0,0)$. Moreover, we have the estimate

$$
\begin{aligned}
\left\|S_{j}\left(x^{\gamma_{3}^{\prime}}\left(t D_{t}\right)^{k^{\prime}} e^{i \theta}\right)\right\|_{L^{\infty}} & =\left\|S_{j}\left(\left(\frac{2 D_{x}}{t}\right)^{\gamma_{3}^{\prime}}\left(\frac{D_{x}^{2}}{t}+\frac{i}{2}\right)^{k^{\prime}} e^{i \theta}\right)\right\|_{L^{\infty}} \\
& \leqslant C\left(\frac{2^{j}}{t}\right)^{\left|\gamma_{3}^{\prime}\right|}\left(1+\frac{2^{j}}{\sqrt{t}}\right)^{2 k^{\prime}} .
\end{aligned}
$$

We conclude that when $\left|\gamma_{1}\right|+\left|\gamma_{2}\right|>0, \gamma_{3}^{\prime}=0$, (4.1.34) is in $E_{\beta, \alpha^{\prime}, 1}^{s-2, s^{\prime}-|\gamma|-1}$ and when $\left(\gamma_{1}, \gamma_{2}\right)=(0,0),\left|\gamma_{3}^{\prime}\right|=1,(4.1 .34)$ is in

$$
E_{\alpha, \alpha^{\prime}+1,1}^{s-2 k, s^{\prime}-|\gamma|-1} \subset E_{\alpha+2, \alpha^{\prime}, 1}^{s-2 k, s^{\prime}-|\gamma|-1} \subset E_{\beta, \alpha^{\prime}, 1}^{s-2, s^{\prime}-|\gamma|-1} .
$$

This is the wanted conclusion, which shows that $W_{1}$ is a quadratic remainder.

Let us show that $W_{2}$ is also a quadratic remainder, proving that for any $\gamma \in \mathbb{N}^{2}$, $|\gamma| \leqslant M$, any $k=0,1$, any $v_{1}, v_{2} \in \mathcal{G}$

$$
\left(t D_{t}\right)^{k} x^{\gamma} R\left(x^{\gamma_{3}} e^{i \theta},(D / t)^{\gamma_{1}} v_{1}(D / t)^{\gamma_{2}} v_{2}\right)
$$

belongs to $E_{\beta, \alpha^{\prime}, 1}^{s-2 k s^{\prime}-|\gamma|-1}$, with estimates by $\left\|v_{1}\right\|_{\mathcal{G}}\left\|v_{2}\right\|_{\mathcal{G}}$. As for (4.1.33), we can write (4.1.35) as a linear combination of quantities

$$
\widetilde{R}\left(\left(t D_{t}\right)^{k^{\prime}} x^{\gamma_{3}^{\prime}} e^{i \theta},\left(t D_{t}\right)^{k^{\prime \prime}} x^{\gamma^{\prime}}(D / t)^{\gamma_{1}} v_{1}(D / t)^{\gamma_{2}} v_{2}\right)
$$

with $k^{\prime}+k^{\prime \prime}=k,\left|\gamma^{\prime}\right| \leqslant|\gamma|+1 \leqslant M+1 \leqslant 2 M-3, \gamma_{3}^{\prime}=0$ if $\left|\gamma_{1}\right|+\left|\gamma_{2}\right|>0,\left|\gamma_{3}^{\prime}\right|=1$ if $\left(\gamma_{1}, \gamma_{2}\right)=(0,0)$. We have seen above that $w=\left(t D_{t}\right)^{k^{\prime \prime}} x^{\gamma^{\prime}}(D / t)^{\gamma_{1}} v_{1}(D / t)^{\gamma_{2}} v_{2}$ belongs 
to $E_{\beta, \alpha^{\prime}, 1}^{s-2 k^{\prime \prime}, s^{\prime}-|\gamma|-1}$ when $\left|\gamma_{1}\right|+\left|\gamma_{2}\right|>0$ and to $E_{\alpha, \alpha^{\prime}, 1}^{s-2 k^{\prime \prime}, s^{\prime}-|\gamma|}$ when $\left(\gamma_{1}, \gamma_{2}\right)=(0,0)$. In the first case, we apply (i) of proposition 3.1.6 with $\kappa_{1}=k^{\prime}, \kappa_{2}=0$ and $\alpha$, (resp. $s$, resp. $s^{\prime}$ ) replaced by $\beta$ (resp. $s-2 k^{\prime \prime}$, resp. $s^{\prime}-|\gamma|-1$ ). By (4.1.6), conditions (3.1.36) are satisfied, so (4.1.36) belongs to $E_{\beta, \alpha^{\prime}, 1}^{s-2 k, s^{\prime}-|\gamma|-1}$. When $\left(\gamma_{1}, \gamma_{2}\right)=(0,0)$, we use (ii) of proposition 3.1.6 with $\kappa_{1}=k^{\prime} \leqslant 1, \kappa_{2}=\gamma_{3}^{\prime}$ and $s$ (resp. $s^{\prime}$ ) replaced by $s-2 k^{\prime \prime}$ (resp. $s^{\prime}-|\gamma|$ ). Since (4.1.6) is satisfied, assumptions (3.1.39) are realized, so (4.1.36) again belongs to $E_{\beta, \alpha^{\prime}, 1}^{s-2 k, s^{\prime}-|\gamma|-1}$. This gives the conclusion.

Finally, let us show that $W_{3}$ gives a contribution to the sum in the right hand side of (4.1.31). This term is the quadratic form associated to the bilinear form

$$
\mathrm{Op}^{\mathcal{B}}\left((D / t)^{\gamma_{1}} v_{1}(D / t)^{\gamma_{2}} v_{2}\right)\left(x^{\gamma_{3}} e^{i \theta}\right) \text {. }
$$

Consider first the case $\gamma_{3}=0$ whence $\left|\gamma_{1}\right|+\left|\gamma_{2}\right|>0$. We write then (4.1.37) as $\mathrm{Op}^{\mathcal{B}}(a(t, x) \otimes b(t, \xi))\left(e^{i \theta}\right)$ where $b \equiv 1 \in \Sigma_{0}^{0}$, and where $a=(D / t)^{\gamma_{1}} v_{1}(D / t)^{\gamma_{2}} v_{2}$ satisfies by lemma 4.1 .5 (i) for $k=0,1,2\left(t D_{t}\right)^{k} a \in \mathcal{E}_{\beta, \alpha^{\prime}, 1}^{s-2 k s^{\prime}-1}\left(M^{\prime}\right)$. In other words, $W_{3}$ is of form $\mathrm{Op}^{\mathcal{B}}\left(A_{1}\right)\left(e^{i \theta}\right)$ for a quadratic symbol $A_{1}$. When $\gamma_{3} \neq 0$, we take $\chi \in C_{0}^{\infty}\left(\mathbb{R}^{2}\right), \chi \equiv 1$ close to 0 and decompose (4.1.37) as

$$
\begin{aligned}
\mathrm{Op}^{\mathcal{B}}\left((D / t)^{\gamma_{1}} v_{1}(D / t)^{\gamma_{2}} v_{2} \chi(\xi / t)\right)\left((2 D / t)^{\gamma_{3}} e^{i \theta}\right) \\
+\mathrm{Op}^{\mathcal{B}}\left((D / t)^{\gamma_{1}} v_{1}(D / t)^{\gamma_{2}} v_{2}(1-\chi)(\xi / t)\right)\left((2 D / t)^{\gamma_{3}} e^{i \theta}\right)
\end{aligned}
$$

The first term in (4.1.38) may be written $\mathrm{Op}^{\mathcal{B}}(a \otimes b)\left(e^{i \theta}\right)$, with $b=\chi(\xi / t)(2 \xi / t)^{\gamma_{3}}$, element of $\Sigma_{1}^{0}$, and $a=(D / t)^{\gamma_{1}} v_{1}(D / t)^{\gamma_{2}} v_{2}$. We just have to use lemma 4.1 .5 (i), to conclude that $\left(t D_{t}\right)^{k} a \in \mathcal{E}_{\alpha, \alpha^{\prime}, 1}^{s-2 k, s^{\prime}-1}\left(M^{\prime}\right)$, i.e. that $a \otimes b$ is a quadratic symbol of wanted type.

The second contribution to (4.1.38) may be written as a linear combination of terms

$$
\widetilde{\mathrm{Op}}^{\mathcal{B}}\left(x^{\gamma_{3}^{\prime}}(D / t)^{\gamma_{1}} v_{1}(D / t)^{\gamma_{2}} v_{2}(1-\chi)(\xi / t)\right)\left(e^{i \theta}\right)
$$

with $\left|\gamma_{3}^{\prime}\right| \leqslant 1$ if $\left|\gamma_{1}\right|+\left|\gamma_{2}\right|>0,\left|\gamma_{3}^{\prime}\right| \leqslant 2$ if $\gamma_{1}=\gamma_{2}=0$. For $\gamma \in \mathbb{N}^{2}$ with $|\gamma| \leqslant M^{\prime}$, we have $|\gamma|+\left|\gamma_{3}^{\prime}\right| \leqslant M^{\prime}+2 \leqslant 2 M-3$. Again, lemma 4.1.5 (i) shows that (4.1.39) may be written $\mathrm{Op}^{\mathcal{B}}(a \otimes b)\left(e^{i \theta}\right)$ with $b=(1-\chi)(\xi / t) \in \Sigma_{1}^{0},\left(t D_{t}\right)^{k} a \in \widetilde{\mathcal{E}}_{\alpha, \alpha^{\prime}, 1}^{s-2 k, s^{\prime}-1}\left(M^{\prime}\right)$ for $k=0,1,2$. This concludes the proof of the lemma.

We study now the contribution to $S_{2}$ in (4.1.15) indexed by $m \in I_{1}-\{0\}$.

Lemma 4.1.7. - Set $M^{\prime}=2 M-5$. Denote by $S_{2}^{\prime}$ the contribution to $S_{2}$ given by the second sum (4.1.15) limited to $m \in I_{1}-\{0\}$. There are quadratic symbols $A_{m}(v, V, t, \xi)$ $m \in I_{1}-\{0\}$, with values in $\widetilde{\mathcal{E}}_{\beta, \alpha^{\prime}, 1}^{s, s^{\prime}-1}\left(M^{\prime}\right) \otimes \Sigma_{0}^{0}+\widetilde{\mathcal{E}}_{\alpha, \alpha^{\prime}, 1}^{s, s^{\prime}-1}\left(M^{\prime}\right) \otimes \Sigma_{1}^{0}$, satisfying (4.1.24), and there is a quadratic remainder $R(v, V)$ satisfying the second condition (4.1.16), such that

$$
S_{2}^{\prime}=\sum_{m \in I_{1}-\{0\}} \operatorname{Op}^{\mathcal{B}}\left(A_{m}(v, V, t, \xi)\right)\left(e^{i m \theta}\right)+R(v, V)
$$


Proof. - The general term of $S_{2}^{\prime}$ may be written

$$
\left((D / t)^{\gamma_{1}} v\right)\left((D / t)^{\gamma_{2}} V\right)\left(x^{\gamma_{3}} e^{i m \theta}\right)
$$

where $\left|\gamma_{1}\right| \leqslant 1,\left|\gamma_{2}\right| \leqslant 1,0<\left|\gamma_{1}\right|+\left|\gamma_{2}\right|+\left|\gamma_{3}\right| \leqslant 2$, and where $v$ (resp. $V$ ) should be read indifferently $v$ or $\bar{v}$ (resp. $V$ or $\bar{V}$ ). We decompose (4.1.41) as

$$
\begin{aligned}
\mathrm{Op}^{\mathcal{B}}\left(x^{\gamma_{3}} e^{i m \theta}\right)\left[\left(\left(\frac{D}{t}\right)^{\gamma_{1}} v\right)\left(\left(\frac{D}{t}\right)^{\gamma_{2}} V\right)\right] & +R\left(x^{\gamma_{3}} e^{i m \theta},\left(\left(\frac{D}{t}\right)^{\gamma_{1}} v\right)\left(\left(\frac{D}{t}\right)^{\gamma_{2}} V\right)\right) \\
& +\mathrm{Op}^{\mathcal{B}}\left(\left(\frac{D}{t}\right)^{\gamma_{1}} v\left(\frac{D}{t}\right)^{\gamma_{2}} V\right)\left(x^{\gamma_{3}} e^{i m \theta}\right) .
\end{aligned}
$$

Remark that the assumption $V \in \mathcal{V}$ implies

$$
\left(t D_{t}\right)^{k} V \in \widetilde{\mathcal{E}}_{\alpha, \alpha^{\prime}, \infty}^{s-2 k, s^{\prime}}(M) \subset \underline{\widetilde{\mathcal{E}}}_{\alpha, \alpha^{\prime}, \infty}^{s-2 k, s^{\prime}}(M), k=0,1,
$$

i.e. $V$ satisfies the same assumptions as $v$. Consequently, the proof of the preceding lemma implies that the first two terms in (4.1.42) provide a contribution to the quadratic remainder $R(v, V)$ (the fact that $v$ admits two $t D_{t}$ derivatives by definition of $\mathcal{G}$, instead of only one for $V$, does not play any role, since this is not used in the study of the first two terms in (4.1.32)). Let us study the last term in (4.1.42). When $\gamma_{3}=0$, so $\left|\gamma_{1}\right|+\left|\gamma_{2}\right|>0$, (ii) of lemma 4.1 .5 implies that for $k=0,1, c \in S^{\mu^{\prime}}$, $\mu^{\prime} \in[0, \mu], a=(D / t)^{\gamma_{1}} v(D / t)^{\gamma_{2}} V$ satisfies

$$
c(\sqrt{t} x)\left(t D_{t}\right)^{k} a \in \widetilde{\mathcal{E}}_{\beta, \alpha^{\prime}, 1}^{s-2 k-\mu^{\prime}, s^{\prime}-1}\left(M^{\prime}\right) .
$$

Consequently, the last term of (4.1.42) is of type $\mathrm{Op}^{\mathcal{B}}(a \otimes b)\left(e^{i m \theta}\right)$ with $b \in \Sigma_{0}^{0}$, i.e. satisfies (4.1.24). When $\gamma_{3} \neq 0$, write the last term in (4.1.42) as

$$
\begin{aligned}
\mathrm{Op}^{\mathcal{B}}\left((D / t)^{\gamma_{1}} v(D / t)^{\gamma_{2}} V \chi(\xi / t)\right)\left((2 D / m t)^{\gamma_{3}} e^{i m \theta}\right) \\
+\mathrm{Op}^{\mathcal{B}}\left((D / t)^{\gamma_{1}} v(D / t)^{\gamma_{2}} V(1-\chi)(\xi / t)\right)\left(x^{\gamma_{3}} e^{i m \theta}\right) .
\end{aligned}
$$

The first term may be written $\operatorname{Op}^{\mathcal{B}}(a \otimes b)\left(e^{i m \theta}\right)$ with a symbol

$$
b(t, \xi)=\chi(\xi / t)(2 \xi / t m)^{\gamma_{3}} \in \Sigma_{1}^{0},
$$

since $\left|\gamma_{3}\right| \geqslant 1$, and with $a$ satisfying

$$
c(\sqrt{t} x)\left(t D_{t}\right)^{k} a \in \widetilde{\mathcal{E}}_{\alpha, \alpha^{\prime}, 1}^{s-2 k-\mu^{\prime}, s^{\prime}-1}\left(M^{\prime}\right)
$$

for $k=0,1, c \in S^{\mu^{\prime}}, \mu^{\prime} \in[0, \mu]$ by (ii) of lemma 4.1.5. The second contribution to (4.1.43) may be written as linear combination of terms

$$
\widetilde{\mathrm{Op}}^{\mathcal{B}}\left(x^{\gamma_{3}^{\prime}}(D / t)^{\gamma_{1}} v(D / t)^{\gamma_{2}} V \chi_{1}(\xi / t)\right)\left(e^{i m \theta}\right)
$$

where $\chi_{1} \in C^{\infty}$ is bounded as well as its derivatives, $\chi_{1} \equiv 0$ close to 0 and $\left|\gamma_{3}^{\prime}\right| \leqslant\left|\gamma_{3}\right|$. This may be written as $\operatorname{Op}^{\mathcal{B}}(a \otimes b)\left(e^{i m \theta}\right)$ with $b=\chi_{1}(\xi / t) \in \Sigma_{1}^{0}$ and $a$ satisfying (4.1.44) because of (ii) of lemma 4.1.5 and of the fact that we have $s^{\prime}>5$, since $s^{\prime}=M+4-s$ and $M>5$. The conclusion of the lemma follows.

Let us examine now the non-oscillating contribution to $S_{2}$. 
LEMMA 4.1.8. - The sum $\sum_{|\gamma| \leqslant 2} P_{\gamma}^{0}\left(v, V, \bar{v}, \bar{V} ; \frac{D}{t} v, \frac{D}{t} V, \frac{D}{t} \bar{v}, \frac{D}{t} \bar{V}\right)$ may be written

$$
\sum_{j=1}^{2} x_{j} H_{j}(v, V)+R(v, V)
$$

where $H_{j}$ satisfies (4.1.25) and $R(v, V)$ is a quadratic remainder.

Proof. - The general term of the expression to be studied is of type

$$
\left((D / t)^{\gamma_{1}} v\right)\left((D / t)^{\gamma_{2}} V\right) x^{\gamma_{3}}
$$

for $\left|\gamma_{1}\right| \leqslant 1,\left|\gamma_{2}\right| \leqslant 1,0<\left|\gamma_{1}\right|+\left|\gamma_{2}\right|+\left|\gamma_{3}\right| \leqslant 2$, and where $v$ (resp. $V$ ) should be read indifferently $v$ or $\bar{v}$ (resp. $V$ or $\bar{V}$ ). When $\left|\gamma_{1}\right|+\left|\gamma_{2}\right|>0$, and so $\left|\gamma_{3}\right| \leqslant 1$, (ii) of lemma 4.1.5 shows that this expression belongs to $\mathcal{G}^{\prime}$, and so provides a contribution to the quadratic remainder $R(v, V)$. When $\gamma_{1}=\gamma_{2}=0$, we have $1 \leqslant\left|\gamma_{3}\right| \leqslant 2$, so we can write (4.1.46) $\sum_{j=1}^{2} x_{j} H_{j}(v, V)$ where $H_{j}$ is of form $x^{\gamma_{3}^{\prime}} v V$ for $\gamma_{3}^{\prime}$ satisfying $\left|\gamma_{3}^{\prime}\right| \leqslant\left|\gamma_{3}\right|-1 \leqslant 1$. By (ii) of lemma 4.1.5, and the assumption $s^{\prime}>5$, which follows from $M>5$, we obtain

$$
c(\sqrt{t} x)\left(t D_{t}\right)^{k} H_{j} \in \widetilde{\mathcal{E}}_{\alpha, \alpha^{\prime}, 1}^{s-2 k-\mu^{\prime}, s^{\prime}}(M+1)
$$

for $c \in S^{\mu^{\prime}}, \mu^{\prime} \in[0, \mu]$, which is the wanted conclusion.

Finally, let us study contribution $S_{3}$ to (4.1.15).

LEMMA 4.1.9. - If $\mu$ is in ]0,2[ close enough to 2, the contribution $S_{3}$ to (4.1.15) is a quadratic remainder $R(V)$ satisfying the last condition (4.1.16).

Proof. - We write $S_{3}$ as a linear combination of expressions

$$
x^{\gamma_{3}}\left((D / t)^{\gamma_{1}} V\right)\left((D / t)^{\gamma_{2}} V\right) e^{i m \theta}
$$

with $m \in I_{2}, 0<\left|\gamma_{1}\right|+\left|\gamma_{2}\right|+\left|\gamma_{3}\right| \leqslant 2$, and where again one should read indifferently $V$ or $\bar{V}$ for $V$. We decompose (4.1.47) as

$$
\begin{aligned}
\mathrm{Op}^{\mathcal{B}}\left(x^{\gamma_{3}} e^{i m \theta}\right)\left[\left(\left(\frac{D}{t}\right)^{\gamma_{1}} V\right)\left(\left(\frac{D}{t}\right)^{\gamma_{2}} V\right)\right] & +R\left(x^{\gamma_{3}} e^{i m \theta},\left(\left(\frac{D}{t}\right)^{\gamma_{1}} V\right)\left(\left(\frac{D}{t}\right)^{\gamma_{2}} V\right)\right) \\
& +\mathrm{Op}^{\mathcal{B}}\left(\left(\frac{D}{t}\right)^{\gamma_{1}} V\left(\frac{D}{t}\right)^{\gamma_{2}} V\right)\left(x^{\gamma_{3}} e^{i m \theta}\right) .
\end{aligned}
$$

We have seen in the proof of lemma 4.1.7 that $V$ satisfies the same assumptions as $v$, and so that the first two terms are quadratic remainders of type $R(V)$. We are thus left with the study of the bilinear term in $\left(V^{\prime}, V^{\prime \prime}\right) \in \mathcal{V} \times \mathcal{V}$

$$
\mathrm{Op}^{\mathcal{B}}\left(\left((D / t)^{\gamma_{1}} V^{\prime}\right)\left((D / t)^{\gamma_{2}} V^{\prime \prime}\right)\right)\left(x^{\gamma_{3}} e^{i m \theta}\right)
$$

When $\gamma_{3}=0$, so $\left|\gamma_{1}\right|+\left|\gamma_{2}\right|>0$, lemma 4.1 .5 (iii) shows that $w=(D / t)^{\gamma_{1}} V^{\prime}(D / t)^{\gamma_{2}} V^{\prime \prime}$ satisfies

$$
\langle\sqrt{t} x\rangle^{2 \mu} w \in \widetilde{\mathcal{E}}_{\beta, \alpha^{\prime}, 1}^{s-2 \mu, s^{\prime}-1}(M),\langle\sqrt{t} x\rangle^{\mu} t D_{t} w \in \widetilde{\mathcal{E}}_{\beta, \alpha^{\prime}, 1}^{s-2-\mu, s^{\prime}-1}(M) .
$$

We can exploit these decay assumptions on $w$ to prove that (4.1.49) is actually a remainder: one checks immediately using (4.1.6) that if $\mu$ is chosen in ]0,2[ close enough to 2, assumption (3.2.87) is satisfied for $w$ (replacing in (3.2.87) $\left(\alpha, \mu, s^{\prime}\right)$ by 
$\left.\left(\beta, 2 \mu, s^{\prime}-1\right)\right)$ and for $t D_{t} w$ (replacing in $(3.2 .87)\left(\alpha, s, s^{\prime}\right)$ by $\left.\left(\beta, s-2, s^{\prime}-1\right)\right)$. We thus deduce from corollary 3.2.7 that

$$
\begin{aligned}
\mathrm{Op}^{\mathcal{B}}(w)\left(e^{i m \theta}\right) & \in \mathcal{E}_{\beta+2, \alpha^{\prime}, 1}^{s, s^{\prime}-1}(M) \subset \mathcal{E}_{\beta, \alpha^{\prime}, 1}^{s, s^{\prime}-1}(M) \\
\mathrm{Op}^{\mathcal{B}}\left(t D_{t} w\right)\left(e^{i m \theta}\right) & \in \mathcal{E}_{\beta+2, \alpha^{\prime}, 1}^{s-2, s^{\prime}-1}(M) \subset \mathcal{E}_{\beta, \alpha^{\prime}, 1}^{s-2, s^{\prime}-1}(M) .
\end{aligned}
$$

Since $\mathrm{Op}^{\mathcal{B}}(w)\left(t D_{t} e^{i m \theta}\right)$ belongs also to this last space, and since the norms in these spaces are estimated by $C\left\|V^{\prime}\right\|_{\mathcal{V}}\left\|V^{\prime \prime}\right\|_{\mathcal{V}}$, this shows that (4.1.49) is a quadratic remainder.

When $\gamma_{3} \neq 0$, we write (4.1.49) as a combination of terms

$$
\begin{gathered}
\mathrm{Op}^{\mathcal{B}}\left(\left((D / t)^{\gamma_{1}} V^{\prime}\right)\left((D / t)^{\gamma_{2}} V^{\prime \prime}\right) \chi(\xi / t)\right)\left[(2 D / m t)^{\gamma_{3}} e^{i m \theta}\right], \\
\mathrm{Op}^{\mathcal{B}}\left(x^{\gamma_{3}^{\prime}}\left((D / t)^{\gamma_{1}} V^{\prime}\right)\left((D / t)^{\gamma_{2}} V^{\prime \prime}\right) \chi_{1}(\xi / t)\right)\left[e^{i m \theta}\right]
\end{gathered}
$$

with $\chi_{1} \in C^{\infty}\left(\mathbb{R}^{d}\right)$, bounded as well as all its derivatives, $\chi_{1} \equiv 0$ close to 0 , and $\left|\gamma_{3}^{\prime}\right| \leqslant\left|\gamma_{3}\right|$. All these expressions can be written as quantities of type $\widetilde{\mathrm{Op}}^{\mathcal{B}}(w(t, x) b(t, \xi))\left(e^{i m \theta}\right)$ with $b \in \Sigma_{1}^{0}$, and $w$ satisfying, because of (iii) of lemma 4.1.5,

$$
\langle\sqrt{t} x\rangle^{2 \mu} w \in \widetilde{\mathcal{E}}_{\alpha, \alpha^{\prime}, 1}^{s-2 \mu s^{\prime}-1}(M),\langle\sqrt{t} x\rangle^{\mu}\left(t D_{t}\right) w \in \widetilde{\mathcal{E}}_{\alpha, \alpha^{\prime}, 1}^{s-2-\mu, s^{\prime}-1}(M) .
$$

Since (3.2.86) (with $s^{\prime}$ replaced by $s^{\prime}-1$ ) is satisfied by (4.1.6), we get from corollary 3.2.7 that (4.1.50) belongs to $\mathcal{E}_{\alpha+4, \alpha^{\prime}, 1}^{s, s^{\prime}-1}(M) \subset \mathcal{E}_{\beta, \alpha^{\prime}, 1}^{s, s^{\prime}-1}(M)$, and its image by $t D_{t}$ to $\mathcal{E}_{\beta, \alpha^{\prime}, 1}^{s-2, s^{\prime}-1}(M)$.

Proposition 4.1.4 now follows from (4.1.2), (4.1.10), (4.1.15) and lemmas 4.1.6 to 4.1.9.

\section{Step 2: Cancelation of main oscillating terms}

We want now to choose $V_{1}, V_{-3}$ to eliminate in the right hand side of (4.1.26) the main oscillating contributions, namely $\mathrm{Op}^{\mathcal{B}}\left(A_{m}\right)\left(e^{i m \theta}\right), m=1,-3$. In the case of example (4.1.20), the oscillating term to get rid of is $T_{\left(v \frac{D_{x}}{t} v\right)} e^{i \theta}$. To do so, we remark that an explicit computation shows that

$$
\left(D_{t}+\frac{D_{x}^{2}}{t^{2}}\right) e^{i \theta}=\frac{1}{t} \Lambda(t, D) e^{i \theta}
$$

where $\Lambda(t, \xi)=2 \frac{\xi^{2}}{t}+i$ is an elliptic symbol of order 2 in $\xi / \sqrt{t}$. We thus set

$$
V_{1}(v)=e^{-i \theta} T_{\left(v \frac{D_{x}}{t} v\right)}\left(\Lambda(t, D)^{-1} e^{i \theta}\right) .
$$

The equality (4.1.51) allows one to prove that modulo remainders

$$
\left(D_{t}+\frac{D_{x}^{2}}{t^{2}}\right)\left[V_{1}(v) e^{i \theta}\right]=\frac{1}{t} T_{\left(v \frac{D_{x}}{t} v\right)} e^{i \theta}
$$

i.e. this choice of $V_{1}$ eliminates the $\mathrm{Op}^{\mathcal{B}}\left(A_{1}\right)\left(e^{i \theta}\right)$ contribution to (4.1.26) (this is the contains of lemma 4.1.11). Moreover $V_{1}$ will satisfy the wanted decay and smoothness requirements: this is established in general in proposition 4.1.10. The idea is that $\Lambda(t, D)^{-1} e^{i \theta}$ in (4.1.52) admits two $D_{x} / \sqrt{t}$-derivatives. But when acting on an 
oscillatory exponential $e^{i \theta}$, a gain of $\left(D_{x} / \sqrt{t}\right)^{-2}$ in smoothness is equivalent to a gain of $\langle\sqrt{t} x\rangle^{-2}$ in decay. Let us now proceed to the general case.

Proposition 4.1.10. - There exists for $m=1,-3$ Lipschitz functions $v \mapsto V_{m}(v)$ defined on the unit ball of $\mathcal{G}$, with values in $\mathcal{V}$, vanishing at 0 , and quadratic symbols $v \mapsto A_{m}^{\prime}(v, t, \xi)$ with values in

$$
\sum_{\ell=0}^{1} \widetilde{\mathcal{E}}_{\beta, \alpha^{\prime}, 1}^{s-2-\ell, s^{\prime}-1}\left(M^{\prime}\right) \otimes \Sigma_{0}^{-2-\ell}+\sum_{\ell=0}^{1} \widetilde{\mathcal{E}}_{\alpha, \alpha^{\prime}, 1}^{s-2, s^{\prime}-1}\left(M^{\prime}\right) \otimes \Sigma_{1}^{-2-\ell}
$$

satisfying

$$
t D_{t} A_{m}^{\prime} \in \sum_{\ell=0}^{1} \widetilde{\mathcal{E}}_{\beta, \alpha^{\prime}, 1}^{s-4, s^{\prime}-1}\left(M^{\prime}\right) \otimes \Sigma_{0}^{-2-\ell}+\sum_{\ell=0}^{1} \widetilde{\mathcal{E}}_{\alpha, \alpha^{\prime}, 1}^{s-4-\ell, s^{\prime}-1}\left(M^{\prime}\right) \otimes \Sigma_{1}^{-2-\ell}
$$

such that for $m=1,-3$

$$
\left(D_{t}+\frac{D_{x}^{2}}{t^{2}}\right)\left(V_{m} e^{i m \theta}\right)=\frac{1}{t} \mathrm{Op}^{\mathcal{B}}\left(A_{m}(v, t, \xi)\right)\left(e^{i m \theta}\right)-\frac{1}{t} \mathrm{Op}^{\mathcal{B}}\left(A_{m}^{\prime}(v, t, \xi)\right)\left(e^{i m \theta}\right) .
$$

We first prove the following lemma.

Lemma 4.1.11. - Let $\ell \in \mathbb{N}, m \in \mathbb{Z}-\{0,-1\}, k \in\{1,2\}, M^{\prime}=2 M-5$.

(i) Let $A(t, x, \xi)$ be a symbol satisfying for $k^{\prime}=0, \cdots, k$

$$
\left(t D_{t}\right)^{k^{\prime}} A \in \widetilde{\mathcal{E}}_{\alpha, \alpha^{\prime}, 1}^{s-\ell-2 k^{\prime}, s^{\prime}-1}\left(M^{\prime}\right) \otimes \Sigma_{1}^{-\ell}
$$

(resp.

$$
\left.\left(t D_{t}\right)^{k^{\prime}} A \in \widetilde{\mathcal{E}}_{\beta, \alpha^{\prime}, 1}^{s-\ell-2 k^{\prime}, s^{\prime}-1}\left(M^{\prime}\right) \otimes \Sigma_{0}^{-\ell}\right) .
$$

There are $B(t, x, \xi), C(t, x, \xi)$ satisfying

$$
\begin{aligned}
& \left(t D_{t}\right)^{k^{\prime}} B \in \sum_{\ell^{\prime}=0}^{1} \widetilde{\mathcal{E}}_{\alpha, \alpha^{\prime}, 1}^{s-\ell-\ell^{\prime}-2 k^{\prime}, s^{\prime}-1}\left(M^{\prime}\right) \otimes \Sigma_{1}^{-\ell-\ell^{\prime}-2}, \quad k^{\prime}=0, \cdots, k \\
& \left(t D_{t}\right)^{k^{\prime}} C \in \sum_{\ell^{\prime}=0}^{1} \widetilde{\mathcal{E}}_{\alpha, \alpha^{\prime}, 1}^{s-\ell-\ell^{\prime}-2 k^{\prime}-2, s^{\prime}-1}\left(M^{\prime}\right) \otimes \Sigma_{1}^{-\ell-\ell^{\prime}-2}, \quad k^{\prime}=0, \cdots, k-1
\end{aligned}
$$

(resp.

$$
\begin{aligned}
& \left(t D_{t}\right)^{k^{\prime}} B \in \sum_{\ell^{\prime}=0}^{1} \widetilde{\mathcal{E}}_{\beta, \alpha^{\prime}, 1}^{s-\ell-\ell^{\prime}-2 k^{\prime}, s^{\prime}-1}\left(M^{\prime}\right) \otimes \Sigma_{0}^{-\ell-\ell^{\prime}-2}, \quad k^{\prime}=0, \cdots, k \\
& \left.\left(t D_{t}\right)^{k^{\prime}} C \in \sum_{\ell^{\prime}=0}^{1} \widetilde{\mathcal{E}}_{\beta, \alpha^{\prime}, 1}^{s-\ell-\ell^{\prime}-2 k^{\prime}-2, s^{\prime}-1}\left(M^{\prime}\right) \otimes \Sigma_{0}^{-\ell-\ell^{\prime}-2}, \quad k^{\prime}=0, \cdots, k-1\right)
\end{aligned}
$$

with $B$ supported in the domain $\{\xi ;|\xi|>c \sqrt{t}\}$, and such that

$$
\left(D_{t}+\frac{D_{x}^{2}}{t^{2}}\right)\left[\mathrm{Op}^{\mathcal{B}}(B)\left(e^{i m \theta}\right)\right]=\frac{1}{t} \mathrm{Op}^{\mathcal{B}}(A)\left(e^{i m \theta}\right)+\frac{1}{t} \mathrm{Op}^{\mathcal{B}}(C)\left(e^{i m \theta}\right) .
$$

Moreover, the semi-norms of $B$ and $C$ are controlled linearly by the semi-norms of $A$. 
(ii) Assume that $A$ satisfies for $k^{\prime}=0,1$, and any $c \in S^{\mu^{\prime}}, \mu^{\prime} \in[0, \mu]$

$$
c(\sqrt{t} x)\left(t D_{t}\right)^{k^{\prime}} A \in \widetilde{\mathcal{E}}_{\alpha, \alpha^{\prime}, 1}^{s-\ell-\mu^{\prime}-2 k^{\prime}, s^{\prime}-1}\left(M^{\prime}\right) \otimes \Sigma_{1}^{-\ell}
$$

(resp.

$$
\left.c(\sqrt{t} x)\left(t D_{t}\right)^{k^{\prime}} A \in \widetilde{\mathcal{E}}_{\beta, \alpha^{\prime}, 1}^{s-\ell-\mu^{\prime}-2 k^{\prime}, s^{\prime}-1}\left(M^{\prime}\right) \otimes \Sigma_{0}^{-\ell}\right) .
$$

Then the symbols $B$ and $C$ satisfy for any $c \in S^{\mu^{\prime}}, \mu^{\prime} \in[0, \mu]$

$$
\begin{aligned}
c(\sqrt{t} x)\left(t D_{t}\right)^{k^{\prime}} B & \in \sum_{\ell^{\prime}=0}^{1} \widetilde{\mathcal{E}}_{\alpha, \alpha^{\prime}, 1}^{s-\ell-\ell^{\prime}-\mu^{\prime}-2 k^{\prime}, s^{\prime}-1}\left(M^{\prime}\right) \otimes \Sigma_{1}^{-\ell-\ell^{\prime}-2}, \quad k^{\prime}=0,1 \\
c(\sqrt{t} x) C & \in \sum_{\ell^{\prime}=0}^{1} \widetilde{\mathcal{E}}_{\alpha, \alpha^{\prime}, 1}^{s-\ell-\ell^{\prime}-\mu^{\prime}-2, s^{\prime}-1}\left(M^{\prime}\right) \otimes \Sigma_{1}^{-\ell-\ell^{\prime}-2},
\end{aligned}
$$

(resp.

$$
\begin{aligned}
c(\sqrt{t} x)\left(t D_{t}\right)^{k^{\prime}} B & \in \sum_{\ell^{\prime}=0}^{1} \widetilde{\mathcal{E}}_{\beta, \alpha^{\prime}, 1}^{s-\ell-\ell^{\prime}-\mu^{\prime}-2 k^{\prime}, s^{\prime}-1}\left(M^{\prime}\right) \otimes \Sigma_{0}^{-\ell-\ell^{\prime}-2}, \quad k^{\prime}=0,1 \\
c(\sqrt{t} x) C & \left.\in \sum_{\ell^{\prime}=0}^{1} \widetilde{\mathcal{E}}_{\beta, \alpha^{\prime}, 1}^{s-\ell-\ell^{\prime}-\mu^{\prime}-2, s^{\prime}-1}\left(M^{\prime}\right) \otimes \Sigma_{0}^{-\ell-\ell^{\prime}-2}\right) .
\end{aligned}
$$

Proof. - The lemma asserts that if $A$ is given, we can find a $B$, which has the same smoothness and a better $\xi / \sqrt{t}$-decay than $A$, so that (4.1.60) is true, with a remainder $C$ that is two degree less regular than $A$ but has a better $\xi / \sqrt{t}$ decay.

Assume that $A$ satisfies (4.1.56). Take $\chi \in C_{0}^{\infty}\left(\mathbb{R}^{2}\right), \chi \equiv 1$ close to 0 and write

$$
A(t, x, \xi)=A(t, x, \xi)(1-\chi(\xi / \sqrt{t}))+A(t, x, \xi) \chi(\xi / \sqrt{t}) .
$$

We incorporate the last term in $C$ i.e. we assume that $A$ vanishes for $|\xi|<c \sqrt{t}$. For $m \in \mathbb{Z}-\{0,-1\}$, set

$$
\Lambda_{m}(t, \xi)=\left(1+\frac{1}{m}\right) \frac{\xi^{2}}{t}+i
$$

so that $\left(D_{t}+\frac{D_{x}^{2}}{t^{2}}\right) e^{i m \theta}=\frac{1}{t} \Lambda_{m}(t, D) e^{i m \theta}$. We define

$$
B(t, x, \xi)=A(t, x, \xi) \Lambda_{m}(t, \xi)^{-1}-2 \frac{D_{x}}{\sqrt{t}} A(t, x, \xi) \cdot \frac{\xi}{\sqrt{t}} \Lambda_{m}(t, \xi)^{-2}
$$

Then $B$ satisfies (4.1.58), and a direct computation shows that

with

$$
\left(D_{t}+\frac{D_{x}^{2}}{t^{2}}\right)\left[\mathrm{Op}^{\mathcal{B}}(B)\left(e^{i m \theta}\right)\right]-\frac{1}{t} \mathrm{Op}^{\mathcal{B}}(A)\left(e^{i m \theta}\right)=\frac{1}{t} \mathrm{Op}^{\mathcal{B}}(C)\left(e^{i m \theta}\right)
$$

$$
C(t, x, \xi)=\left(t D_{t}+\frac{D_{x}^{2}}{t}\right)(B(t, x, \xi))-4 \frac{D_{x}^{2}}{t} A(t, x, \xi)\left(\frac{\xi}{\sqrt{t}}, \frac{\xi}{\sqrt{t}}\right) \Lambda_{m}^{-2}
$$

which satisfies (4.1.58). The proof under assumption (4.1.57) is identical. Assertion (ii) of the lemma follows from the above expressions for $B$ and $C$. 
REMARK. - We use in the above proof the assumption $m \neq-1$ in an essential way. If $m=-1, \Lambda_{m}(t, \xi)$ is no longer of order 2 , and we can no longer make the asymptotic construction in the proof of the above lemma. In other words we are not able to eliminate oscillatory terms of type $\mathrm{Op}^{\mathcal{B}}(A)\left(e^{-i \theta}\right)$. These terms do not appear in the right hand side of our equation (4.1.26) because of the restrictive assumption on the form of the nonlinearity in the right hand side of (1.1.2).

Proof of proposition 4.1.10. - We apply lemma 4.1.11 (i) to the symbol $A=$ $A_{m}, m=1,-3$, with $k=2, \ell=0$. We denote by $-A_{m}^{\prime}$ the symbols $C$ of lemma 4.1.11: they satisfy conditions (4.1.53), (4.1.54). Consequently, if we set

$$
V_{m}=e^{-i m \theta} \mathrm{Op}^{\mathcal{B}}\left(B_{m}\right)\left(e^{i m \theta}\right)
$$

where $B_{m}$ satisfies (4.1.58), or (4.1.59), we just have to see that $V_{m}$ is a Lipschitz function of $v$ on the unit ball of $\mathcal{G}$, with values in $\mathcal{V}$. The Lipschitz dependence will follow from the fact that $A_{m}$, and thus $B_{m}$, are Lipschitz in $v$ by construction. The main fact to prove is that $V_{m}$ fulfills the conditions (4.1.8) defining $\mathcal{V}$. We will make use of proposition 3.2.2. Consider first the case corresponding to (4.1.58) i.e.

$$
\left(t D_{t}\right)^{k^{\prime}} B_{m} \in \sum_{\ell=0}^{1} \widetilde{\mathcal{E}}_{\alpha, \alpha^{\prime}, 1}^{s-\ell-2 k^{\prime}, s^{\prime}-1}\left(M^{\prime}\right) \otimes \Sigma_{1}^{-\ell-2} .
$$

We apply proposition 3.2.2 with $\rho=2$ or $\rho=3, \nu=1, b \equiv 1, \mu=0$. We check that because of (4.1.7), condition (3.2.4) and the second condition (3.2.6) are satisfied. We deduce from (3.2.7) that

$$
V_{m} \in \widetilde{E}_{\alpha+2-0, \alpha^{\prime}, 1}^{s+2, s^{\prime}-1} \subset \widetilde{E}_{\beta, \alpha^{\prime}, 1}^{s+2, s^{\prime}-1} .
$$

Writing for $\gamma \in \mathbb{N}^{2},|\gamma| \leqslant M, x^{\gamma} V_{m}$ as a linear combination of expressions $e^{-i m \theta} \mathrm{Op}^{\mathcal{B}}\left(x^{\gamma^{\prime}} B_{m}\right)\left(e^{i m \theta}\right)$ for $\left|\gamma^{\prime}\right| \leqslant|\gamma|$, and using (4.1.67), we get in the same way that $V_{m} \in \widetilde{\mathcal{E}}_{\beta, \alpha^{\prime}, 1}^{s+2, s^{\prime}-1}(M)$.

Let us study now the derivative

$$
t D_{t} V_{m}=e^{-i m \theta} \mathrm{Op}^{\mathcal{B}}\left(t D_{t} B_{m}\right)\left(e^{i m \theta}\right)-e^{-i m \theta}\left[m t x^{2} / 4, \mathrm{Op}^{\mathcal{B}}\left(B_{m}\right)\right]\left(e^{i m \theta}\right) .
$$

Using (4.1.67) and proposition 3.2.2, we get that the first term in the right hand side belongs to $\widetilde{\mathcal{E}}_{\beta, \alpha^{\prime}, 1}^{s, s^{\prime}-1}(M)$. Going back to the definition of $\mathrm{Op}^{\mathcal{B}}(\cdot)$ we see that the last term in (4.1.68) is the opposite of

$$
e^{-i m \theta} \sum_{j} S_{j-1} B_{m}(t, x, D)\left[m t x^{2} / 4, \Delta_{j}\right]\left(e^{i m \theta}\right) .
$$

If for $\Delta_{j}=\varphi\left(2^{-j} D\right)$, we set $\Delta_{j}^{(1)}=(\nabla \varphi)\left(2^{-j} D\right), \Delta_{j}^{(2)}=(\Delta \varphi)\left(2^{-j} D\right)$, we have that

$$
\left[\frac{m t x^{2}}{4}, \Delta_{j}\right]\left(e^{i m \theta}\right)=\left[-\frac{m}{4}\left(2^{-j} \sqrt{t}\right)^{2} \Delta_{j}^{(2)}+i \Delta_{j}^{(1)} \cdot\left(2^{-j} D\right)\right]\left(e^{i m \theta}\right) .
$$


Since $B_{m}$ is supported in $\{|\xi|>c \sqrt{t}\}$, the sum in (4.1.69) is restricted to $2^{j}>c \sqrt{t}$, so (4.1.70) shows that (4.1.69) is of type $e^{-i m \theta} \widetilde{\mathrm{Op}}^{\mathcal{B}}\left(B_{m}\right)\left(e^{i m \theta}\right)$, and so belongs to $\widetilde{\mathcal{E}}_{\beta, \alpha^{\prime}, 1}^{s+2, s^{\prime}-1}(M) \subset \widetilde{\mathcal{E}}_{\beta, \alpha^{\prime}, 1}^{s, s^{\prime}-1}(M)$.

To study

$$
c(\sqrt{t} x)(D / t)^{\gamma}\left(t D_{t}\right)^{k} V_{m}, \quad k=0,1,|\gamma| \leqslant 1
$$

with $c \in S^{\mu}$, we argue as above when $\gamma=0$ : we apply proposition 3.2 .2 remarking that in (3.2.7) we can take $\zeta>0$ since $\mu<2$. We get that (4.1.71) belongs to $\widetilde{\mathcal{E}}_{\alpha, \alpha^{\prime}, 1}^{s+2-\mu-2 k, s^{\prime}-1}(M)$. When $|\gamma|=1$ in (4.1.71), we write this expression as

$$
(D / t)^{\gamma}\left[c(\sqrt{t} x)\left(t D_{t}\right)^{k} V_{m}\right]+\frac{1}{\sqrt{t}} c_{1}(\sqrt{t} x)\left(t D_{t}\right)^{k} V_{m}
$$

where $c_{1} \in S^{\mu-1}$. The first contribution belongs to

$$
\widetilde{\mathcal{E}}_{\alpha, \alpha^{\prime}+|\gamma|, 1}^{s+2-\mu-2 k, s^{\prime}-1-|\gamma|}(M) \subset \widetilde{\mathcal{E}}_{\alpha+2|\gamma|, \alpha^{\prime}, 1}^{s+2-\mu-2 k, s^{\prime}-1-|\gamma|}(M)
$$

which is the wanted conclusion. To study the second contribution, we apply again proposition 3.2 .2 with $\mu$ replaced by $\mu-1$. In this case, in (3.2.7), we can take $\zeta=1$ since $\mu-1<1$ whence $c_{1}(\sqrt{t} x)\left(t D_{t}\right)^{k} V_{m} \in \widetilde{\mathcal{E}}_{\alpha+1, \alpha^{\prime}, 1}^{s+2-(\mu-1)-2 k, s^{\prime}-1}(M)$. Coming back to the definitions of these spaces, we deduce that the second term in (4.1.72) belongs to the same space as the first one.

When instead of (4.1.67) one assumes that

$$
\left(t D_{t}\right)^{k^{\prime}} B_{m} \in \sum_{\ell=0}^{1} \widetilde{\mathcal{E}}_{\beta, \alpha^{\prime}, 1}^{s-\ell-2 k^{\prime}, s^{\prime}-1}\left(M^{\prime}\right) \otimes \Sigma_{0}^{-\ell-2}
$$

(i.e. when we study the case corresponding to (4.1.59)), we use proposition 3.2.2 with $\nu=0$, and $\alpha$ replaced by $\beta$. We remark that the first condition (3.2.6), which reads $\frac{\beta}{2}+\alpha^{\prime}>0, \beta+\alpha^{\prime}+1>0$ is satisfied by (4.1.6). We deduce from (3.2.7) with $\zeta=0+$ (which is possible as $\frac{d}{2}-\beta-\alpha^{\prime}>0$ ) that

$$
\left(t D_{t}\right)^{k} V_{m} \in \widetilde{\mathcal{E}}_{\beta, \alpha^{\prime}, 1}^{s+2-2 k, s^{\prime}-1}(M)
$$

which is the wanted conclusion. The study of (4.1.71) when $\gamma=0$ is similar, and shows that this term belongs to $\widetilde{\mathcal{E}}_{\beta, \alpha^{\prime}, 1}^{s+2-\mu-2 k, s^{\prime}-1}(M)$. When $|\gamma|=1$, we use decomposition (4.1.72). The first term will belong to

$$
\widetilde{\mathcal{E}}_{\beta, \alpha^{\prime}+1,1}^{s+2-\mu-2 k, s^{\prime}-1-|\gamma|}(M) \subset \widetilde{\mathcal{E}}_{\beta+2, \alpha^{\prime}, 1}^{s+2-\mu-2 k, s^{\prime}-1-|\gamma|}(M)
$$

which gives the conclusion as $\beta+2>\alpha+2$. To study the second contribution to (4.1.72), we remark first that

$$
c_{1}(\sqrt{t} x)\left(t D_{t}\right)^{k} V_{m} \in \widetilde{\mathcal{E}}_{\beta, \alpha^{\prime}, 1}^{s+2-(\mu-1)-2 k, s^{\prime}-1}(M) \subset \widetilde{\mathcal{E}}_{\alpha+1, \alpha^{\prime}, 1}^{s+2-\mu-2 k, s^{\prime}-1}(M)
$$

again by (3.2.7) and since (4.1.6) implies $\beta>\alpha+1$. Exploiting the $1 / \sqrt{t}$ factor in the second term of (4.1.72), we see that this expression belongs to $\widetilde{\mathcal{E}}_{\alpha+2, \alpha^{\prime}, 1}^{s+2-\mu-2 k, s^{\prime}-1-|\gamma|}(M)$. This concludes the proof. 
Corollary 4.1.12. - There exists:

- for $m=1,-3$ Lipschitz functions $v \mapsto V_{m}(v)$ defined on the unit ball of $\mathcal{G}$, with values in $\mathcal{V}$, vanishing at $v=0$,

- for $m=1,-3$ quadratic symbols $v \mapsto A_{m}^{\prime}(v, t, \xi)$ with values in (4.1.53), satisfying (4.1.54),

- for $m \in I_{1}-\{0\}$, quadratic symbols $v \mapsto A_{m}^{\prime}(v, t, \xi)$ satisfying for $k=0,1$, $c \in S^{\mu^{\prime}}, \mu^{\prime} \in[0, \mu]$

$$
\begin{aligned}
c(\sqrt{t} x)\left(t D_{t}\right)^{k} A_{m}^{\prime}(v, t, \xi) & \\
& \in \widetilde{\mathcal{E}}_{\beta, \alpha^{\prime}, 1}^{s-2 k-\mu^{\prime}, s^{\prime}-1}\left(M^{\prime}\right) \otimes \Sigma_{0}^{0}+\widetilde{\mathcal{E}}_{\alpha, \alpha^{\prime}, 1}^{s-2 k-\mu^{\prime}, s^{\prime}-1}\left(M^{\prime}\right) \otimes \Sigma_{1}^{0},
\end{aligned}
$$

- A function $v \mapsto H(v)=\sum_{j=1}^{2} x_{j} H_{j}(v)$ where $H_{j}(v)$ are defined on the unit ball of $\mathcal{G}$, vanish at 0 , and satisfy for $k=0,1, c \in S^{\mu^{\prime}}, \mu^{\prime} \in[0, \mu], v, v^{\prime}$ in this unit ball

$$
\begin{aligned}
\left\|c(\sqrt{t} x)\left(t D_{t}\right)^{k}\left[H_{j}(v)-H_{j}\left(v^{\prime}\right)\right]\right\|_{\widetilde{\mathcal{E}}_{\alpha, \alpha^{\prime}, 1}^{s-\mu^{\prime}-2 k, s^{\prime}}(M+1)} & \leqslant C\left(\|v\|_{\mathcal{G}}+\left\|v^{\prime}\right\|_{\mathcal{G}}\right)\left\|v-v^{\prime}\right\|_{\mathcal{G}},
\end{aligned}
$$

- A quadratic remainder $v \mapsto R(v)$ defined on the unit ball of $\mathcal{G}$, with values in $\mathcal{G}^{\prime}$, such that, if we set $I=\{1,-3\} \cup\left(I_{1}-\{0\}\right)=\{-4,-3,-2,2,1\}$ and

$$
F(v)=\sum_{m \in I} \mathrm{Op}^{\mathcal{B}}\left(A_{m}^{\prime}(v, t, \xi)\right)\left(e^{i m \theta}\right)+H(v)+R(v),
$$

for any element $v$ of the unit ball of $\mathcal{G}$ which is a solution of

$$
\left(D_{t}+\frac{D_{x}^{2}}{t^{2}}\right) v=\frac{1}{t} F(v),
$$

the function $w$ given by (4.1.10) is a solution to (4.1.2).

Proof. - We define $V_{m}(v)$ by proposition 4.1.10 and denote $V(v)=\left(V_{1}(v), V_{-3}(v)\right)$. We just have to see that if $v$ satisfies (4.1.77) with a convenient definition of $A_{m}^{\prime}, H, R$ in (4.1.76), then $(v, V)$ is a solution to (4.1.26). By (4.1.55), the first sum in the right hand side of (4.1.26) is $\frac{1}{t} \sum_{m \in\{1,-3\}} \mathrm{Op}^{\mathcal{B}}\left(A_{m}^{\prime}\right)\left(e^{i m \theta}\right)$. For $m \in I_{1}-\{0\}$ we define $A_{m}^{\prime}(v, t, \xi)=A_{m}(v, V, t, \xi)$. Since $v \mapsto V(v)$ is Lipschitz with values in $\mathcal{V}$, (4.1.24) implies that $A_{m}^{\prime}$ satisfies (4.1.74) for $m \in I_{1}-\{0\}$. We define also the function $H_{j}(v)$ satisfying (4.1.75) from the bilinear forms of (4.1.25) by the formula $H_{j}(v)=H_{j}(v, V(v))$. Since $v \mapsto V(v)$ is Lipschitz, (4.1.75) follows from (4.1.25). In the same way, if we substitute $V(v)$ into the last term $R(v, V)$ of (4.1.26), we get a quadratic remainder. A solution $v$ to (4.1.77) thus allows us to construct a solution $(v, V)$ to (4.1.26), and so a solution $w$ to (4.1.2).

Our next task is to find a global solution to (4.1.77) for small enough Cauchy data. We cannot do that directly on equation (4.1.77): actually we want to find a solution $v$, which belongs in particular to $\underline{E}_{\alpha, \alpha^{\prime}, \infty}^{s, s^{\prime}}$. In the right hand side, $R(v)$ belongs to 
$E_{\beta, \alpha^{\prime}, 1}^{s, s^{\prime}-1} \subset E_{\alpha, \alpha^{\prime}, 1}^{s, s^{\prime}-1} \subset F_{\alpha, \alpha^{\prime}, 1}^{s, s^{\prime}-1 / 2}$. The smoothing property of theorem 2.2.3 tells us that the corresponding linear solution belongs to $\underline{E}_{\alpha, \alpha^{\prime}, \infty}^{s, s^{\prime}}$, which is nice. The trouble comes from the $\mathrm{Op}^{\mathcal{B}}\left(A_{m}^{\prime}\right)\left(e^{i m \theta}\right)$ terms in (4.1.76). We cannot hope to put these terms in $F_{\alpha, \alpha^{\prime}, 1}^{s, s^{\prime}-1 / 2}$. In fact, when $m=1,-3, A_{m}^{\prime}$ belongs to $\Sigma_{0}^{-2}$ : this means that the gain of smoothness we can get when applying $\mathrm{Op}^{\mathcal{B}}\left(A_{m}^{\prime}\right)$ to $e^{i m \theta}$ is at most 2 i.e. these terms cannot be in any $F_{\alpha, \alpha^{\prime}, 1}^{s, s^{\prime}-1 / 2}$ space for $s>2$. In the same way, for $m \in I_{1}-\{0\}$, $A_{m}^{\prime}$ is a symbol of order 0 , which decays like $\langle\sqrt{t} x\rangle^{-2+0}$ relatively to space. Since the action of $D_{x} / \sqrt{t}$ over $e^{i m \theta}$ results in a loss of $\sqrt{t} x$, this shows that the best that can be expected for $\mathrm{Op}^{\mathcal{B}}\left(A_{m}^{\prime}\right)\left(e^{i m \theta}\right)\left(m \in I_{1}\right)$ is to lie in a $F_{\alpha, \alpha^{\prime}, 1}^{s, s^{\prime}-1 / 2}$ space with $s<2$. On the other hand, because of conditions (4.1.6), we do need to be able to solve our problem in a space of that type with $s>2$ (actually $s$ close to 4 ). Because of that we will exploit again the fact that the phases of oscillations in (4.1.76) are non-characteristic ones to further reduce the equation.

\section{Step 3: Last reduction of the equation}

Up to now, we have eliminated by an appropriate choice of $V$ the first line in the right hand side of (4.1.26). We still have to eliminate the $\mathrm{Op}^{\mathcal{B}}\left(A_{m}(v, V, t, \xi)\right)\left(e^{i m \theta}\right)$ terms (or equivalently, the terms of type $\mathrm{Op}^{\mathcal{B}}\left(A_{m}^{\prime}(v, t, \xi)\right)\left(e^{i m \theta}\right)$ in $\left.(4.1 .76)\right)$. In the case of example (4.1.20) these are coming from the rectangular terms in (4.1.21) i.e. are expressions of type $\mathrm{Op}^{\mathcal{B}}\left(A_{2}(v, V)\right)\left(e^{2 i \theta}\right)$, where $A_{2}(v, V)$ is bilinear. In particular, this $A_{2}$ decays like $\langle\sqrt{t} x\rangle^{-2}$, which means that the corresponding term admits two $D_{x} / \sqrt{t}$-derivatives. This is not enough to incorporate it to the remainders in (4.1.26), since the latter have to accept about four of these derivatives. In this third step, we thus repeat the procedure of step 2, to get rid of these last annoying terms.

Proposition 4.1.13. - There exists a function $v \mapsto K(v)$ (resp. $v \mapsto \widetilde{R}(v)$ ) defined on the unit ball of $\mathcal{G}$, with values in $\widetilde{\mathcal{E}}_{\alpha, \alpha^{\prime}, 1}^{s, s^{\prime}}(M)$ (resp. $\mathcal{E}_{\beta, \alpha^{\prime}, 1}^{s, s^{\prime}}(M)$ ), such that for any $v, v^{\prime}$ in the unit ball of $\mathcal{G}$

$$
\begin{aligned}
&\left\|K(v)-K\left(v^{\prime}\right)\right\|_{\widetilde{\mathcal{E}}_{\widetilde{\alpha}^{\prime, \alpha^{\prime}, 1}}^{s, M}(M)} \leqslant C\left\|v-v^{\prime}\right\|_{\mathcal{G}}\left(\|v\|_{\mathcal{G}}+\left\|v^{\prime}\right\|_{\mathcal{G}}\right) \\
&\left\|\widetilde{R}(v)-\widetilde{R}\left(v^{\prime}\right)\right\|_{\mathcal{E}_{\beta, \alpha^{\prime}, 1}^{s, s^{\prime}}(M)} \leqslant C\left\|v-v^{\prime}\right\|_{\mathcal{G}}\left(\|v\|_{\mathcal{G}}+\left\|v^{\prime}\right\|_{\mathcal{G}}\right),
\end{aligned}
$$

and satisfying the following: if $v$ and $v^{\prime}$ are two elements of the unit ball of $\mathcal{G}$, solutions to

$$
\left(D_{t}+\frac{D_{x}^{2}}{t^{2}}\right)\left(v-K\left(v^{\prime}\right)\right)=\frac{1}{t} H\left(v^{\prime}\right)+\frac{1}{t} \widetilde{R}\left(v^{\prime}\right)
$$

then $\left(v, v^{\prime}\right)$ solves

$$
\left(D_{t}+\frac{D_{x}^{2}}{t^{2}}\right) v=\frac{1}{t} F\left(v^{\prime}\right)
$$

where $F$ and $H$ are defined in (4.1.76). 
Proof. - We have to define $K\left(v^{\prime}\right)$ in such a way that the contribution

$$
\sum_{m \in I} \mathrm{Op}^{\mathcal{B}}\left(A_{m}^{\prime}\right)\left(e^{i m \theta}\right)
$$

in (4.1.76) is canceled. Apply first lemma 4.1.11 (i) to $A_{m}^{\prime}, m=1,-3$. Since then $A_{m}^{\prime}$ belongs to (4.1.53), and $t D_{t} A_{m}^{\prime}$ to (4.1.54), we get from this lemma symbols $B_{m}^{\prime}$ and $C_{m}^{\prime}$ satisfying for $m=1,-3$

$$
\begin{aligned}
& B_{m}^{\prime} \in \sum_{\ell=0}^{2} \widetilde{\mathcal{E}}_{\beta, \alpha^{\prime}, 1}^{s-2-\ell, s^{\prime}-1}\left(M^{\prime}\right) \otimes \Sigma_{0}^{-4-\ell}+\sum_{\ell=0}^{2} \widetilde{\mathcal{E}}_{\alpha, \alpha^{\prime}, 1}^{s-2-\ell, s^{\prime}-1}\left(M^{\prime}\right) \otimes \Sigma_{1}^{-4-\ell} \\
& C_{m}^{\prime} \in \sum_{\ell=0}^{2} \widetilde{\mathcal{E}}_{\beta, \alpha^{\prime}, 1}^{s-4-\ell, s^{\prime}-1}\left(M^{\prime}\right) \otimes \Sigma_{0}^{-4-\ell}+\sum_{\ell=0}^{2} \widetilde{\mathcal{E}}_{\alpha, \alpha^{\prime}, 1}^{s-4-\ell s^{\prime}-1}\left(M^{\prime}\right) \otimes \Sigma_{1}^{-4-\ell} .
\end{aligned}
$$

When $m \in I_{1}-\{0\}, A_{m}^{\prime}$ satisfies (4.1.74) and lemma 4.1.11 (ii) provides symbols $B_{m}^{\prime}$, $C_{m}^{\prime}$ such that for $c \in S^{\mu^{\prime}}, \mu^{\prime} \in[0, \mu]$

$$
\begin{aligned}
& c(\sqrt{t} x) B_{m}^{\prime} \in \sum_{\ell=0}^{1} \widetilde{\mathcal{E}}_{\beta, \alpha^{\prime}, 1}^{s-\mu^{\prime}-\ell, s^{\prime}-1}\left(M^{\prime}\right) \otimes \Sigma_{0}^{-2-\ell}+\sum_{\ell=0}^{1} \widetilde{\mathcal{E}}_{\alpha, \alpha^{\prime}, 1}^{s-\mu^{\prime}-\ell, s^{\prime}-1}\left(M^{\prime}\right) \otimes \Sigma_{1}^{-2-\ell} \\
& c(\sqrt{t} x) C_{m}^{\prime} \in \sum_{\ell=0}^{1} \widetilde{\mathcal{E}}_{\beta, \alpha^{\prime}, 1}^{s-\mu^{\prime}-2-\ell, s^{\prime}-1}\left(M^{\prime}\right) \otimes \Sigma_{0}^{-2-\ell}+\sum_{\ell=0}^{1} \widetilde{\mathcal{E}}_{\alpha, \alpha^{\prime}, 1}^{s-\mu^{\prime}-2-\ell, s^{\prime}-1}\left(M^{\prime}\right) \otimes \Sigma_{1}^{-2-\ell} .
\end{aligned}
$$

With these notations, we set

$$
\begin{aligned}
K\left(v^{\prime}\right) & =\sum_{m \in I} \mathrm{Op}^{\mathcal{B}}\left(B_{m}^{\prime}\right)\left(e^{i m \theta}\right) \\
R_{1}\left(v^{\prime}\right) & =\sum_{m \in I} \mathrm{Op}^{\mathcal{B}}\left(C_{m}^{\prime}\right)\left(e^{i m \theta}\right)
\end{aligned}
$$

and (4.1.60) shows that (4.1.80) implies (4.1.79) if we can prove that $K\left(v^{\prime}\right)$ belongs to $\widetilde{\mathcal{E}}_{\alpha, \alpha^{\prime}, 1}^{s, s^{\prime}}(M)$ and $R_{1}\left(v^{\prime}\right)$ to $\mathcal{E}_{\beta, \alpha^{\prime}, 1}^{s, s^{\prime}-1}(M)$ (the quadratic estimates (4.1.78) follow then from the linear dependence of (4.1.83) in $B_{m}^{\prime}, C_{m}^{\prime}$, and the quadratic nature of these terms).

Consider first the contribution to $K\left(v^{\prime}\right)$ coming from terms of type $\mathrm{Op}^{\mathcal{B}}\left(B_{m}^{\prime}\right)\left(e^{i m \theta}\right)$, $m=1,-3$. Condition (4.1.81) implies

$$
B_{m}^{\prime} \in \sum_{\ell=0}^{2} \widetilde{\mathcal{E}}_{\beta, \alpha^{\prime}, 1}^{s-4, s^{\prime}}\left(M^{\prime}\right) \otimes \Sigma_{0}^{-4-\ell}+\sum_{\ell=0}^{2} \widetilde{\mathcal{E}}_{\alpha, \alpha^{\prime}, 1}^{s-4-\ell, s^{\prime}}\left(M^{\prime}\right) \otimes \Sigma_{1}^{-4-\ell} .
$$

We apply corollary 3.2.7 with $b \in \Sigma_{0}^{-4-\ell}$ (resp. $b \in \Sigma_{1}^{-4-\ell}$ ), $\mu=0, s$ replaced by $s-4-\ell, M$ replaced by $M^{\prime}=2 M-5$, and we check that conditions (3.2.87), (3.2.86) which read respectively

$$
\begin{gathered}
-1<\beta+\alpha^{\prime}, \quad s-4-\ell<\alpha^{\prime}-1, \quad s+s^{\prime}-4-\ell-M^{\prime}<-1 / 2 \\
-2<\alpha+\alpha^{\prime}<-1, \quad s-4-\ell<\alpha+2 \alpha^{\prime}, \quad s+s^{\prime}-4-\ell-M^{\prime}<-1 / 2
\end{gathered}
$$


are satisfied because of (4.1.6). We deduce that the corresponding contribution to $K\left(v^{\prime}\right)$ belongs to $\mathcal{E}_{\alpha+2, \alpha^{\prime}, 1}^{s, s^{\prime}}(M)$. Since $\frac{1}{2}(\alpha+2)+\alpha^{\prime}>0$, the last assertion of lemma 3.1.2 implies that this space equals $\widetilde{\mathcal{E}}_{\alpha+2, \alpha^{\prime}, 1}^{s, s^{\prime}}(M) \subset \widetilde{\mathcal{E}}_{\alpha, \alpha^{\prime}, 1}^{s, s^{\prime}}(M)$. Let us examine now the contribution to $K\left(v^{\prime}\right)$ coming from $\mathrm{Op}^{\mathcal{B}}\left(B_{m}^{\prime}\right)\left(e^{i m \theta}\right), m \in I_{1}-\{0\}$. We deduce from (4.1.82) that for $c \in S^{\mu}$

$$
c(\sqrt{t} x) B_{m}^{\prime} \in \sum_{\ell=0}^{1} \widetilde{\mathcal{E}}_{\beta, \alpha^{\prime}, 1}^{s-\mu-2-\ell, s^{\prime}}\left(M^{\prime}\right) \otimes \Sigma_{0}^{-2-\ell}+\sum_{\ell=0}^{1} \widetilde{\mathcal{E}}_{\alpha, \alpha^{\prime}, 1}^{s-\mu-2-\ell, s^{\prime}}\left(M^{\prime}\right) \otimes \Sigma_{1}^{-2-\ell} .
$$

We apply corollary 3.2.7. We check that conditions (3.2.87), (3.2.86) are satisfied if $\mu \in] 0,2[$ is taken close enough to 2 , because of (4.1.6). We obtain that the corresponding contribution to $K\left(v^{\prime}\right)$ is in $\mathcal{E}_{\alpha+2, \alpha^{\prime}, 1}^{s, s^{\prime}}(M) \subset \widetilde{\mathcal{E}}_{\alpha, \alpha^{\prime}, 1}^{s, s^{\prime}}(M)$.

Next, let us consider $R_{1}\left(v^{\prime}\right)$. To study the contribution coming from $\mathrm{Op}^{\mathcal{B}}\left(C_{m}^{\prime}\right)\left(e^{i m \theta}\right)$, $m=1,-3$, we use assumption (4.1.81) together with corollary 3.2.7. We get that these terms belong to $\mathcal{E}_{\beta, \alpha^{\prime}, 1}^{s, 1}(M)$. For the contribution of $\mathrm{Op}^{\mathcal{B}}\left(C_{m}^{\prime}\right)\left(e^{i m \theta}\right), m \in I_{1}-\{0\}$, we use (4.1.82) and corollary 3.2.7 and get the same conclusion. The proposition is proved.

To close this chapter, we prove a lemma concerning the quantity $F(v)$ defined in (4.1.76).

Lemma 4.1.14. - Denote by $S(v)$ the difference $S(v)=F(v)-H(v)$. For $v, v^{\prime}$ in the unit ball of $\mathcal{G}$, we have the following inequalities:

$$
\begin{aligned}
& \sum_{k=0}^{1}\left\|\left(t D_{t}\right)^{k}\left(S(v)-S\left(v^{\prime}\right)\right)\right\|_{\widetilde{\mathcal{E}}_{\beta, \alpha^{\prime}, 1}^{s-2-2 k, s^{\prime}}(M)} \leqslant C\left\|v-v^{\prime}\right\|_{\mathcal{G}}\left(\|v\|_{\mathcal{G}}+\left\|v^{\prime}\right\|_{\mathcal{G}}\right) \\
& \sum_{k=0}^{1}\left\|\left(t D_{t}\right)^{k}\left(F(v)-F\left(v^{\prime}\right)\right)\right\|_{\widetilde{\mathcal{E}}_{\alpha, \alpha^{\prime}, 1}^{s-2, s^{\prime}}(M)} \leqslant C\left\|v-v^{\prime}\right\|_{\mathcal{G}}\left(\|v\|_{\mathcal{G}}+\left\|v^{\prime}\right\|_{\mathcal{G}}\right) .
\end{aligned}
$$

Proof. - In (4.1.76) we have, because of the definition (4.1.8) of $\mathcal{G}^{\prime}$,

$$
\begin{aligned}
R(v) \in \mathcal{E}_{\beta, \alpha^{\prime}, 1}^{s, s^{\prime}-1}(M) & =\widetilde{\mathcal{E}}_{\beta, \alpha^{\prime}, 1}^{s, s^{\prime}-1}(M) \subset \widetilde{\mathcal{E}}_{\beta, \alpha^{\prime}, 1}^{s-2, s^{\prime}}(M) \\
t D_{t} R(v) \in \mathcal{E}_{\beta, \alpha^{\prime}, 1}^{s-2, s^{\prime}-1}(M) & =\widetilde{\mathcal{E}}_{\beta, \alpha^{\prime}, 1}^{s-2, s^{\prime}-1}(M) \subset \widetilde{\mathcal{E}}_{\beta, \alpha^{\prime}, 1}^{s-4, s^{\prime}}(M)
\end{aligned}
$$

where the equality between $\mathcal{E}$ and $\widetilde{\mathcal{E}}$ spaces follows from $\frac{\beta}{2}+\alpha^{\prime}>0$ and lemma 3.1.2. Consequently, the contributions of $R(v)$ to the left hand sides of (4.1.84) satisfy the wanted conclusion.

Since $H(v) \in \widetilde{\mathcal{E}}_{\alpha, \alpha^{\prime}, 1}^{s, s^{\prime}}(M) \subset \widetilde{\mathcal{E}}_{\alpha, \alpha^{\prime}, 1}^{s-2, s^{\prime}}(M), t D_{t} H(v) \in \widetilde{\mathcal{E}}_{\alpha, \alpha^{\prime}, 1}^{s-2, s^{\prime}-1}(M) \subset \widetilde{\mathcal{E}}_{\alpha, \alpha^{\prime}, 1}^{s-4, s^{\prime}}(M)$ by (4.1.75), the contribution of $H(v)$ to the left hand side of the second inequality (4.1.84) satisfies also the inequality. We are thus reduced to the study of 
$\sum_{m \in I} \mathrm{Op}^{\mathcal{B}}\left(A_{m}^{\prime}(v, t, \xi)\right)\left(e^{i m \theta}\right)$. For $m=1,-3,(4.1 .53)$ and (4.1.54) imply

$$
\left(t D_{t}\right)^{k} A_{m}^{\prime} \in \sum_{\ell=0}^{1} \widetilde{\mathcal{E}}_{\beta, \alpha^{\prime}, 1}^{s-2 k-\ell, s^{\prime}}\left(M^{\prime}\right) \otimes \Sigma_{0}^{-2-\ell}+\sum_{\ell=0}^{1} \widetilde{\mathcal{E}}_{\alpha, \alpha^{\prime}, 1}^{s-4-2 k-\ell, s^{\prime}}\left(M^{\prime}\right) \otimes \Sigma_{1}^{-2-\ell} .
$$

We apply corollary 3.2 .7 with $\mu=0, s$ replaced by $s-4-2 k-\ell, M$ replaced by $M^{\prime}$, and $\alpha$ replaced by $\beta$ for the contributions of the first sum in (4.1.85). One checks that (3.2.86) and (3.2.87) (with $\alpha$ replaced by $\beta$ ) are satisfied because of (4.1.6), whence the conclusion

$$
\begin{aligned}
\mathrm{Op}^{\mathcal{B}}\left(\left(t D_{t}\right)^{k} A_{m}^{\prime}\right)\left(e^{i m \theta}\right) & \in \mathcal{E}_{\beta+2, \alpha^{\prime}, 1}^{s-2-2 k, s^{\prime}}(M)+\mathcal{E}_{\alpha+4, \alpha^{\prime}, 1}^{s-2-2, s^{\prime}}(M) \\
& \subset \mathcal{E}_{\beta, \alpha^{\prime}, 1}^{s-2-2 k, s^{\prime}}(M)=\widetilde{\mathcal{E}}_{\beta, \alpha^{\prime}, 1}^{s-2-2 k, s^{\prime}}(M) .
\end{aligned}
$$

Since $\mathrm{Op}^{\mathcal{B}}\left(A_{m}^{\prime}\right)\left(t D_{t} e^{i m \theta}\right)$ belongs also to $\widetilde{\mathcal{E}}_{\beta, \alpha^{\prime}, 1}^{s-4, s^{\prime}}(M)$, as one sees writing $t D_{t}\left(e^{i m \theta}\right)=$ $\frac{1}{m}\left(i m+\frac{D_{x}^{2}}{t}\right) e^{i m \theta}$ and applying again corollary 3.2.7, we see that the contributions of $\left(t D_{t}\right)^{k} \mathrm{Op}^{\mathcal{B}}\left(A_{m}^{\prime}\right)\left(e^{i m \theta}\right), m=1,-3$ to $\left(t D_{t}\right)^{k} S(v), k=0,1$ belong to $\widetilde{\mathcal{E}}_{\beta, \alpha^{\prime}, 1}^{s-2-2 k, s^{\prime}}(M)$. The fact that these contributions satisfy the first inequality (4.1.84) follows from the quadratic nature of $A_{m}^{\prime}$.

Let us consider now $\operatorname{Op}^{\mathcal{B}}\left(A_{m}^{\prime}\right)\left(e^{i m \theta}\right)$ for $m \in I_{1}-\{0\}$. By (4.1.74), for $k=0,1$, $\mu^{\prime} \in[0, \mu]$

$$
\text { (4.1.86) }\langle\sqrt{t} x\rangle^{\mu^{\prime}}\left(t D_{t}\right)^{k} A_{m}^{\prime} \in \widetilde{\mathcal{E}}_{\beta, \alpha^{\prime}, 1}^{s-\mu^{\prime}-2 k-2, s^{\prime}}\left(M^{\prime}\right) \otimes \Sigma_{0}^{0}+\widetilde{\mathcal{E}}_{\alpha, \alpha^{\prime}, 1}^{s-\mu^{\prime}-2 k-2, s^{\prime}}\left(M^{\prime}\right) \otimes \Sigma_{1}^{0} \text {. }
$$

We apply again corollary 3.2.7 with $s$ replaced by $s-2 k-2, m_{2}=0, \alpha$ replaced by $\beta$ for the contribution of the first term in the right hand side of (4.1.86). If $\mu \in] 0,2[$ is close enough to 2, conditions (3.2.86), (3.2.87) are satisfied by (4.1.6), and we obtain

$$
\mathrm{Op}^{\mathcal{B}}\left(\left(t D_{t}\right)^{k} A_{m}^{\prime}\right)\left(e^{i m \theta}\right) \in \widetilde{\mathcal{E}}_{\beta, \alpha^{\prime}, 1}^{s-2-2 k, s^{\prime}}(M)
$$

which again gives the wanted conclusion.

\subsection{Existence of the global solution}

The main remaining step in the proof of theorem 1.1.1 is to prove the following result: denote by $\mathcal{G}_{0}$ the space

$$
\begin{aligned}
\mathcal{G}_{0} & =\left\{v \in H^{M+4}\left(\mathbb{R}^{2}\right) ; \forall \gamma,|\gamma| \leqslant M,(D+x / 2)^{\gamma} v \in H^{M+4-|\gamma|}\right\} \\
& =\left\{v \in H^{M+4}\left(\mathbb{R}^{2}\right) ; \forall \gamma,|\gamma| \leqslant M, x^{\gamma} v \in H^{M+4-|\gamma|}\right\}
\end{aligned}
$$

THEOREM 4.2.1. - There is $\varepsilon_{0}>0$ such that for any $v_{0} \in \mathcal{G}_{0}$ with $\left\|v_{0}\right\|_{\mathcal{G}_{0}}<\varepsilon_{0}$, the problem

$$
\begin{aligned}
\left(D_{t}+\frac{D_{x}^{2}}{t^{2}}\right)(v-K(v)) & =\frac{1}{t} H(v)+\frac{1}{t} \widetilde{R}(v) \\
\left.v\right|_{t=1} & =v_{0}
\end{aligned}
$$

has a global solution $v \in \mathcal{G}$. 
We shall construct $v$ using the standard iterative scheme

$$
\begin{aligned}
\left(D_{t}+\frac{D_{x}^{2}}{t^{2}}\right)\left(v^{\nu+1}-K\left(v^{\nu}\right)\right) & =\frac{1}{t} H\left(v^{\nu}\right)+\frac{1}{t} \widetilde{R}\left(v^{\nu}\right) \\
\left.v^{\nu+1}\right|_{t=1} & =v_{0}
\end{aligned}
$$

for $\nu \geqslant 0, v^{0}$ being defined as the solution to $\left(D_{t}+\frac{D_{x}^{2}}{t^{2}}\right) v^{0}=0,\left.v^{0}\right|_{t=1}=v_{0}$. The main step is to prove:

Proposition 4.2.2. - There is a constant $C>0$ such that if $v^{\nu}$ belongs to the unit ball of $\mathcal{G}, v^{\nu+1}$ satisfies

$$
\begin{aligned}
\left\|v^{\nu+1}\right\|_{\mathcal{G}} & \leqslant C\left[\left\|v_{0}\right\|_{\mathcal{G}_{0}}+\left\|v^{\nu}\right\|_{\mathcal{G}}^{2}\right] \quad(\nu \geqslant 0) \\
\left\|v^{\nu+1}-v^{\nu}\right\|_{\mathcal{G}} & \leqslant C\left\|v^{\nu}-v^{\nu-1}\right\|_{\mathcal{G}}\left(\left\|v^{\nu-1}\right\|_{\mathcal{G}}+\left\|v^{\nu}\right\|_{\mathcal{G}}\right) \quad(\nu \geqslant 1) .
\end{aligned}
$$

The above inequalities imply in a standard way the existence of a global solution $v \in \mathcal{G}$ to (4.2.2) if the Cauchy data are taken small enough. To prove the proposition, we will study successively the different components of the norms of $\mathcal{G}$.

Lemma 4.2.3. - The norm $\left\|v^{\nu+1}\right\|_{\underline{\mathcal{E}}_{\alpha, \alpha^{\prime}, \infty}^{s, s^{\prime}}(M)}$ (resp. the norm $\left.\left\|v^{\nu+1}-v^{\nu}\right\|_{\underline{\mathcal{E}}_{\alpha, \alpha^{\prime}, \infty}^{s, s^{\prime}}(M)}\right)$ is estimated by the right hand side of the first (resp. second) inequality (4.2.3).

Proof. - Since $Z$ commutes to $D_{t}+\frac{D_{x}^{2}}{t^{2}}$, we deduce from (4.2.2) that for any $\gamma \in$ $\mathbb{N}^{2},|\gamma| \leqslant M$

$$
\left(D_{t}+\frac{D_{x}^{2}}{t^{2}}\right)\left(Z^{\gamma} v^{\nu+1}-Z^{\gamma} K\left(v^{\nu}\right)\right)=\frac{1}{t} Z^{\gamma} H\left(v^{\nu}\right)+\frac{1}{t} Z^{\gamma} \widetilde{R}\left(v^{\nu}\right) .
$$

Since by corollary 4.1.12 $H\left(v^{\nu}\right) \in \widetilde{\mathcal{E}}_{\alpha, \alpha^{\prime}, 1}^{s, s^{\prime}-1}(M)$ and $\widetilde{R}\left(v^{\nu}\right) \in \mathcal{E}_{\beta, \alpha^{\prime}, 1}^{s, s^{\prime}-1}(M)$, the characterization (4.1.5) of these spaces shows that $Z^{\gamma} H\left(v^{\nu}\right)$ and $Z^{\gamma} \widetilde{R}\left(v^{\nu}\right)$ belong to $E_{\alpha, \alpha^{\prime}, 1}^{s, s^{\prime}-|\gamma|-1} \subset F_{\alpha, \alpha^{\prime}, 1}^{s, s^{\prime}-|\gamma|-\frac{1}{2}}$, their norms in these spaces being controlled by $C\left\|v^{\nu}\right\|_{\mathcal{G}}^{2}$. The smoothing inequality (2.2.15) of theorem 2.2.3 implies

$$
\begin{aligned}
\left\|Z^{\gamma}\left(v^{\nu+1}-K\left(v^{\nu}\right)\right)\right\|_{\underline{E}_{\alpha, \alpha^{\prime}, \infty}^{s, s^{\prime}-\gamma \mid}} & \leqslant C\left[\left\|\left.Z^{\gamma}\left(v^{\nu+1}-K\left(v^{\nu}\right)\right)\right|_{t=1}\right\|_{H^{s+s^{\prime}-|\gamma|}}\right. \\
& \left.+\left\|Z^{\gamma} H\left(v^{\nu}\right)\right\|_{F_{\alpha, \alpha^{\prime}, 1}^{s, s^{\prime}-|\gamma|-\frac{1}{2}}}+\left\|Z^{\gamma} \widetilde{R}\left(v^{\nu}\right)\right\|_{F_{\alpha, \alpha^{\prime}, 1}^{s, s^{\prime}-|\gamma|-\frac{1}{2}}}\right]
\end{aligned}
$$

if the inequalities (2.2.13) are satisfied i.e. $\frac{\alpha}{2}+\alpha^{\prime}<0, s+\alpha \geqslant 0, s^{\prime}-|\gamma|+\alpha^{\prime} \geqslant 0$. The first two of these inequalities follow from (4.1.6). Since $|\gamma| \leqslant M=s+s^{\prime}-4$ the last one is satisfied if $s \leqslant \alpha^{\prime}+4$, which follows also from (4.1.6). The last two norms in the right hand side of (4.2.4) are controlled by $C\left(\left\|H\left(v^{\nu}\right)\right\|_{\widetilde{\mathcal{E}}_{\alpha, \alpha^{\prime}, 1}^{s, s^{\prime}-1}(M)}+\left\|\widetilde{R}\left(v^{\nu}\right)\right\|_{\mathcal{E}_{\alpha, \alpha^{\prime}, 1}^{s, s^{\prime}}(M)}\right)$ and so by $C\left\|v^{\nu}\right\|_{\mathcal{G}}^{2}$ because of the properties of $H$ and $\widetilde{R}$ listed in (4.1.75) and (4.1.78). 
In the first term of the right hand side we have

$$
\begin{aligned}
\left\|\left.Z^{\gamma} v^{\nu+1}\right|_{t=1}\right\|_{H^{s+s^{\prime}-|\gamma|}} & =\left\|\left(D_{x}+\frac{x}{2}\right)^{\gamma} v_{0}\right\|_{H^{s+s^{\prime}-|\gamma|}} \leqslant\left\|v_{0}\right\|_{\mathcal{G}_{0}} \\
\left\|\left.Z^{\gamma} K\left(v^{\nu}\right)\right|_{t=1}\right\|_{H^{s+s^{\prime}-|\gamma|}} & \leqslant\left\|Z^{\gamma} K\left(v^{\nu}\right)\right\|_{E_{\alpha, \alpha^{\prime}, \infty}^{s, s^{\prime}-|\gamma|}} \\
& \leqslant\left\|K\left(v^{\nu}\right)\right\|_{\widetilde{\mathcal{E}}_{\left.\widetilde{\mathcal{C}}_{\alpha, \alpha^{\prime}, 1}, M\right)} \leqslant C\left\|v^{\nu}\right\|_{\mathcal{G}}^{2} .}^{2}
\end{aligned}
$$

Consequently, (4.2.4) implies

$$
\left\|Z^{\gamma} v^{\nu+1}\right\|_{\underline{E}_{\alpha, \alpha^{\prime}, \infty}^{s, s^{\prime}|\gamma|}} \leqslant\left\|Z^{\gamma} K\left(v^{\nu}\right)\right\|_{\underline{E}_{\alpha, \alpha^{\prime}, \infty}^{s, s^{\prime}-|\gamma|}}+\left\|v_{0}\right\|_{\mathcal{G}_{0}}+C\left\|v^{\nu}\right\|_{\mathcal{G}}^{2}
$$

which implies the wanted estimate for $\left\|v^{\nu+1}\right\|_{\mathcal{E}_{\alpha, \alpha^{\prime}, \infty}^{s, s^{\prime}}(M)}$ using again inequality (4.1.78) and the injection $\mathcal{E}_{\alpha, \alpha^{\prime}, \infty}^{s, s^{\prime}}(M) \subset \underline{\mathcal{E}}_{\alpha, \alpha^{\prime}, \infty}^{s, s^{\prime}}(M)$. The estimate for $v^{\nu+1}-v^{\nu}$ is obtained in the same way using the equation

$$
\begin{aligned}
\left(D_{t}+\frac{D_{x}^{2}}{t^{2}}\right)\left(v^{\nu+1}-v^{\nu}-\left(K\left(v^{\nu}\right)\right.\right. & \left.\left.-K\left(v^{\nu-1}\right)\right)\right) \\
& =\frac{1}{t}\left(H\left(v^{\nu}\right)-H\left(v^{\nu-1}\right)\right)+\frac{1}{t}\left(\widetilde{R}\left(v^{\nu}\right)-\widetilde{R}\left(v^{\nu-1}\right)\right) .
\end{aligned}
$$

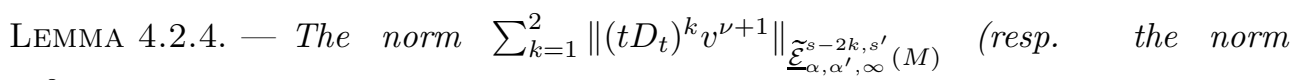
$\left.\sum_{k=1}^{2}\left\|\left(t D_{t}\right)^{k}\left(v^{\nu+1}-v^{\nu}\right)\right\|_{{\underline{\mathcal{E}_{\alpha, \alpha^{\prime}, \infty}^{s}}}^{s-2 k, s^{\prime}}(M)}\right)$ is estimated by the right hand side of the first (resp. second) inequality (4.2.3).

Proof. - By proposition 4.1.13, a solution $\left(v^{\nu}, v^{\nu+1}\right)$ of (4.2.2) solves also

$$
\left(D_{t}+\frac{D_{x}^{2}}{t^{2}}\right) v^{\nu+1}=\frac{1}{t} F\left(v^{\nu}\right)
$$

whence commuting $Z^{\gamma},|\gamma| \leqslant M$ to the equation

$$
\begin{aligned}
t D_{t}\left(Z^{\gamma} v^{\nu+1}\right) & =-\frac{D_{x}^{2}}{t}\left(Z^{\gamma} v^{\nu+1}\right)+Z^{\gamma} F\left(v^{\nu}\right) \\
\left(t D_{t}\right)^{2}\left(Z^{\gamma} v^{\nu+1}\right) & =-\left(t D_{t}\right) \frac{D_{x}^{2}}{t}\left(Z^{\gamma} v^{\nu+1}\right)+t D_{t}\left(Z^{\gamma} F\left(v^{\nu}\right)\right) .
\end{aligned}
$$

We have

$$
\frac{D_{x}^{2}}{t}\left(Z^{\gamma} v^{\nu+1}\right) \in \underline{E}_{\alpha+2, \alpha^{\prime}, \infty}^{s-2, s^{\prime}-|\gamma|}=\underline{\widetilde{E}}_{\alpha+2, \alpha^{\prime}, \infty}^{s-2, s^{\prime}-|\gamma|} \subset \underline{\widetilde{E}}_{\alpha, \alpha^{\prime}, \infty}^{s-2, s^{\prime}-|\gamma|}
$$

when $v^{\nu+1} \in \underline{\mathcal{E}}_{\alpha, \alpha^{\prime}, \infty}^{s, s^{\prime}}(M)$. Consequently, $\left\|\frac{D_{x}^{2}}{t}\left(Z^{\gamma} v^{\nu+1}\right)\right\|_{\underline{\underline{E}}_{\alpha, \alpha^{\prime}, \infty}^{s-2, s^{\prime}-|\gamma|}}$ is controlled in terms of $\left\|v^{\nu+1}\right\|_{\underline{\mathcal{E}}_{\alpha, \alpha^{\prime}, \infty}^{s, s^{\prime}}(M)}$, and so, by lemma 4.2.3, by the right hand side of the first inequality (4.2.3). By lemma 4.1.14, the quantity $\left\|Z^{\gamma} F\left(v^{\nu}\right)\right\|_{\widetilde{E}_{\alpha, \alpha^{\prime}, \infty}^{s-2, s^{\prime}-|\gamma|}}$ is also controlled by $C\left\|v^{\nu}\right\|_{\mathcal{G}}^{2}$. The first equality (4.2.6) thus shows that $\left\|t D_{t} v^{\nu+1}\right\|_{\underline{\mathcal{E}}_{\alpha, \alpha^{\prime}, \infty}^{s-s^{\prime}}(M)}$ is estimated by the wanted quantity. One studies in the same way $\left(t D_{t}\right)^{\frac{2}{\alpha} v^{\nu+1}}$ and $\left(t D_{t}\right)^{k}\left(v^{\nu+1}-v^{\nu}\right), k=1,2$. 
The preceding lemma shows that to estimate the left hand side of (4.2.3) we just need to bound $\left\|v^{\nu+1}\right\|_{\widetilde{\mathcal{E}}_{\alpha, \alpha^{\prime}, \infty}^{s, s^{\prime}}(M)}$ and $\left\|v^{\nu+1}-v^{\nu}\right\|_{{\widetilde{\mathcal{E}_{\alpha, \alpha^{\prime}, \infty}}}^{s, s^{\prime}}(M)}$ instead of the $\underline{\mathcal{E}}_{\alpha, \alpha^{\prime}, \infty}^{s, s^{\prime}}(M)$ norms which have been yet studied in lemma 4.2.3. In other words, we must consider for $|\gamma| \leqslant M$

$$
\begin{gathered}
\left\|\chi(D / \sqrt{t})\left(Z^{\gamma} v^{\nu+1}\right)\right\|_{L^{\infty}(d t d x)} \\
\left\|\chi(D / \sqrt{t})\left(Z^{\gamma}\left(v^{\nu+1}-v^{\nu}\right)\right)\right\|_{L^{\infty}(d t d x)} .
\end{gathered}
$$

LEMMA 4.2.5. - The first (resp. second) norm (4.2.7) is estimated by the right hand side of the first (resp. second) inequality (4.2.3).

Proof. - We shall prove that there is $\delta>0$, and for any $\gamma \in \mathbb{N}^{2}$ with $|\gamma| \leqslant M$, a constant $C>0$, such that for any $t \geqslant 1$

$$
\begin{gathered}
\left|D_{t}\left[\chi(D / \sqrt{t}) Z^{\gamma} v^{\nu+1}\right]\right| \leqslant \frac{C}{t^{1+\delta}}\left[\left\|v_{0}\right\|_{\mathcal{G}_{0}}+\left\|v^{\nu}\right\|_{\mathcal{G}}^{2}\right], \\
\left|D_{t}\left[\chi(D / \sqrt{t}) Z^{\gamma}\left(v^{\nu+1}-v^{\nu}\right)\right]\right| \leqslant \frac{C}{t^{1+\delta}}\left\|v^{\nu}-v^{\nu-1}\right\|_{\mathcal{G}}\left(\left\|v^{\nu}\right\|_{\mathcal{G}}+\left\|v^{\nu-1}\right\|_{\mathcal{G}}\right) .
\end{gathered}
$$

The control of (4.2.7) by the right hand side of (4.2.3) will follow from the time integration of the above inequalities (and the fact that at $t=1$ we have a trivial estimate for $\left.\chi(D)\left(D_{x}+\frac{x}{2}\right)^{\gamma} v_{0}\right)$.

To prove (4.2.8), we deduce from (4.2.5) the equalities

$$
\begin{aligned}
& D_{t}\left[\chi(D / \sqrt{t}) Z^{\gamma} v^{\nu+1}\right]=\frac{i}{2 t} \frac{D}{\sqrt{t}} \cdot \chi^{\prime}\left(\frac{D}{\sqrt{t}}\right) Z^{\gamma} v^{\nu+1} \\
& \quad-\frac{1}{t} \chi\left(\frac{D}{\sqrt{t}}\right) \frac{D_{x}^{2}}{t} Z^{\gamma} v^{\nu+1}+\frac{1}{t} \chi\left(\frac{D}{\sqrt{t}}\right) Z^{\gamma} F\left(v^{\nu}\right) \\
& \begin{aligned}
& D_{t}\left[\chi(D / \sqrt{t}) Z^{\gamma}\left(v^{\nu+1}-v^{\nu}\right)\right]=\frac{i}{2 t} \frac{D}{\sqrt{t}} \cdot \chi^{\prime}\left(\frac{D}{\sqrt{t}}\right) Z^{\gamma}\left(v^{\nu+1}-v^{\nu}\right) \\
&-\frac{1}{t} \chi\left(\frac{D}{\sqrt{t}}\right) \frac{D_{x}^{2}}{t} Z^{\gamma}\left(v^{\nu+1}-v^{\nu}\right)+\frac{1}{t} \chi\left(\frac{D}{\sqrt{t}}\right) Z^{\gamma}\left(F\left(v^{\nu}\right)-F\left(v^{\nu-1}\right)\right) .
\end{aligned}
\end{aligned}
$$

We have that $\frac{D}{\sqrt{t}} \cdot \chi^{\prime}\left(\frac{D}{\sqrt{t}}\right) Z^{\gamma} v^{\nu+1}$ and $\chi\left(\frac{D}{\sqrt{t}}\right) \frac{D_{x}^{2}}{t} Z^{\gamma} v^{\nu+1}$ belong to the space $\underline{E}_{\alpha+2, \alpha^{\prime}, \infty}^{+\infty,+\infty}$. Since $\frac{\alpha}{2}+1+\alpha^{\prime}>0$, property (3.1.6) of lemma 3.1.2 implies that the modulus of the first two terms in the right hand side of (4.2.9) is bounded by $C t^{-1-\delta}\left\|v^{\nu+1}\right\|_{\underline{\mathcal{E}}_{\alpha, \alpha^{\prime}, \infty}^{s,(M)}}$, and so by the right hand side of the first inequality (4.2.8), using lemma 4.2.3. One has a similar statement for the first two contributions to the right hand side of (4.2.10). Let us study now the contribution of $F\left(v^{\nu}\right)$, $F\left(v^{\nu}\right)-F\left(v^{\nu-1}\right)$. We decomposed in lemma 4.1.14 $F\left(v^{\nu}\right)=H\left(v^{\nu}\right)+S\left(v^{\nu}\right)$ with $S\left(v^{\nu}\right) \in \widetilde{\mathcal{E}}_{\beta, \alpha^{\prime}, 1}^{s-2, s^{\prime}}(M)$ whence $\chi(D / \sqrt{t}) Z^{\gamma} S\left(v^{\nu}\right) \in E_{\beta, \alpha^{\prime}, \infty}^{+\infty,+\infty}$. Using $\beta / 2+\alpha^{\prime}>0$ and property (3.1.6) we get for $\frac{1}{t}\left|\chi\left(\frac{D}{\sqrt{t}}\right) Z^{\gamma} S\left(v^{\nu}\right)\right|$ an estimate by the right hand side 
of the first equation (4.2.8). A similar statement holds true for the contributions of $S\left(v^{\nu}\right)-S\left(v^{\nu-1}\right)$ to $(4.2 .10)$. To conclude the proof, we need to estimate

$$
\begin{gathered}
\frac{1}{t}\left|\chi(D / \sqrt{t}) Z^{\gamma} H\left(v^{\nu}\right)\right| \\
\frac{1}{t}\left|\chi(D / \sqrt{t}) Z^{\gamma}\left(H\left(v^{\nu}\right)-H\left(v^{\nu-1}\right)\right)\right| .
\end{gathered}
$$

Using the definition of $Z$, we can write

$$
Z^{\gamma} H\left(v^{\nu}\right)=x^{\gamma} H\left(v^{\nu}\right)+\sum_{\substack{\gamma^{\prime}, \gamma^{\prime \prime}, \ell \\\left|\gamma^{\prime}\right|+\left|\gamma^{\prime \prime}\right|+\ell \leqslant|\gamma| \\\left|\gamma^{\prime \prime}\right|+\ell>0}} t^{-\ell} a_{\gamma^{\prime}, \gamma^{\prime \prime}, \ell}\left(D_{x} / t\right)^{\gamma^{\prime \prime}}\left(x^{\gamma^{\prime}} H\left(v^{\nu}\right)\right)
$$

for convenient coefficients $a_{\gamma^{\prime}, \gamma^{\prime \prime}, \ell}$. By corollary 4.1.12, we know that $H\left(v^{\nu}\right) \in$ $\widetilde{\mathcal{E}}_{\alpha, \alpha^{\prime}, 1}^{s, s^{\prime}-1}(M)$, whence

$$
t^{-\ell}\left(D_{x} / t\right)^{\gamma^{\prime \prime}}\left(x^{\gamma^{\prime}} H\left(v^{\nu}\right)\right) \in \widetilde{E}_{\alpha, \alpha^{\prime}+\left|\gamma^{\prime \prime}\right|+\ell, 1}^{s, s^{\prime}-|\gamma|-1} \subset \widetilde{E}_{\alpha+2, \alpha^{\prime}, 1}^{s, s^{\prime}-|\gamma|-1}
$$

if $\left|\gamma^{\prime \prime}\right|+\ell \geqslant 1$. We then deduce from (3.1.6) the inequality, for $\left|\gamma^{\prime}\right|+\left|\gamma^{\prime \prime}\right|+\ell \leqslant|\gamma|$, $\left|\gamma^{\prime \prime}\right|+\ell>0$

$$
\left|\chi(D / \sqrt{t}) t^{-\ell}(D / t)^{\gamma^{\prime \prime}}\left(x^{\gamma^{\prime}} H\left(v^{\nu}\right)\right)\right| \leqslant \frac{C}{t^{\delta}}\left\|H\left(v^{\nu}\right)\right\|_{\widetilde{\mathcal{E}}_{\alpha, \alpha^{\prime}, 1}^{s, s^{\prime}-1}(M)} \leqslant \frac{C}{t^{\delta}}\left\|v^{\nu}\right\|_{\mathcal{G}}^{2}
$$

for some $\delta>0$, where the last inequality comes from (4.1.75). To estimate (4.2.11) by the right hand side of the first inequality (4.2.8), we just have to study the first term in the right hand side of (4.2.13). Write $H(v)=\sum_{j=1}^{2} x_{j} H_{j}(v)$. We know by (4.1.75) that $\langle\sqrt{t} x\rangle H_{j}(v) \in \widetilde{\mathcal{E}}_{\alpha, \alpha^{\prime}, 1}^{s-1, s^{\prime}}(M+1)$. We shall deduce below from that the inequality

$$
\left|x^{\gamma} H_{j}\left(v^{\nu}\right)\right| \leqslant C\langle\sqrt{t} x\rangle^{-1}\left\|v^{\nu}\right\|_{\mathcal{G}}^{2} .
$$

This will imply

$$
\begin{aligned}
\left|\chi(D / \sqrt{t}) x_{j} x^{\gamma} H_{j}\left(v^{\nu}\right)\right| & \leqslant\left\|x_{j}\langle\sqrt{t} x\rangle^{-1}\right\|_{L^{\infty}(d x)}\left\|v^{\nu}\right\|_{\mathcal{G}}^{2} \\
& \leqslant C t^{-1 / 2}\left\|v^{\nu}\right\|_{\mathcal{G}}^{2} .
\end{aligned}
$$

This shows that the contribution of $x^{\gamma} H\left(v^{\nu}\right)$ to the left hand side of (4.2.11) is controlled by the right hand side of the first equation (4.2.8).

To prove (4.2.14), write that $w=\langle\sqrt{t} x\rangle x^{\gamma} H_{j}(v) \in \widetilde{E}_{\alpha, \alpha^{\prime}, 1}^{s-1, s^{\prime}-|\gamma|}$ and decompose

$$
\phi_{q}(x) w=\phi_{q}(x) \chi(D / \sqrt{t}) w+\sum_{q^{\prime}} \sum_{k ; 2^{k}>c \sqrt{t}}\left(\phi_{q}(x) \widetilde{\Delta}_{k} \widetilde{\phi}_{q^{\prime}}\right)\left(\phi_{q^{\prime}} \Delta_{k} w\right) .
$$

By definition of $\widetilde{E}_{\alpha, \alpha^{\prime}, 1}^{s-1, s^{\prime}-|\gamma|}$, the first term is smaller than $C c_{q}\left\|v^{\nu}\right\|_{\mathcal{G}}^{2}$ for a $\ell^{1}$ sequence $\left(c_{q}\right)_{q}$. The modulus of the second contribution is bounded, using the definition of 
$H_{\alpha, \alpha^{\prime}, 1}^{s-1, s^{\prime}-|\gamma|}$ by

$$
\begin{aligned}
C \sum_{q^{\prime}}\left\langle q-q^{\prime}\right\rangle^{-N} \sum_{k ; 2^{k}>c \sqrt{t}} 2^{k}\left\|\phi_{q^{\prime}} \Delta_{k} w(t, \cdot)\right\|_{L^{2}(d x)} \\
\leqslant C\left\|v^{\nu}\right\|_{\mathcal{G}}^{2} \sum_{q^{\prime}}\left\langle q-q^{\prime}\right\rangle^{-N}\left(\sum_{k ; c \sqrt{t}<2^{k}<t} 2^{k} c_{k q^{\prime}}\left(\frac{2^{k}}{\sqrt{t}}\right)^{-s+1}\left(\frac{2^{k}}{t}\right)^{\alpha^{\prime}}\right. \\
\left.+\sum_{k ; t<2^{k}} 2^{k} c_{k q^{\prime}}\left(\frac{2^{k}}{\sqrt{t}}\right)^{-s+1}\left(\frac{2^{k}}{t}\right)^{-s^{\prime}+|\gamma|}\right)
\end{aligned}
$$

for a $\ell_{k}^{2} \ell_{q^{\prime}}^{1}$ sequence $\left(c_{k q^{\prime}}\right)_{k q^{\prime}}$. Since by (4.1.6) $s>\alpha^{\prime}+2>3, s+s^{\prime}-|\gamma|-2 \geqslant$ $s+s^{\prime}-M-2 \geqslant 2$, we bound (4.2.15) by a $\ell_{q}^{1}$ sequence times $\left\|v^{\nu}\right\|_{\mathcal{G}}^{2}$, whence (4.2.14).

The corresponding estimate for (4.2.12) being obtained in the same way, this concludes the proof of lemma 4.2.5.

Lemmas 4.2.3, 4.2.4, and 4.2.5 imply immediately proposition 4.2 .2 , by definition of the norm on $\mathcal{G}$. As remarked earlier, theorem 4.2.1 is then an immediate consequence of proposition 4.2.2.

Proof of theorem 1.1.1. - If we are given $v_{0} \in \mathcal{G}_{0}$ with small enough norm in that space, theorem 4.2.1 provides a solution $v \in \mathcal{G}$ defined for $t \geqslant 1$ to (4.2.1). By proposition 4.1.13, $v$ is then a solution to

$$
\left(D_{t}+\frac{D_{x}^{2}}{t^{2}}\right) v=\frac{1}{t} F(v)
$$

and by corollary 4.1.12, the function $w=v+V_{1}(v) e^{i \theta}+V_{-3}(v) e^{-3 i \theta}$ is a solution to (4.1.2), hence to (4.1.1), defined for $t \geqslant 1$. Since this in turn implies theorem 1.1.1, the only thing we still have to prove is that for any $w_{0} \in \mathcal{H}^{M+4}$ in a small enough neighborhood of 0 , there is $v_{0}$ in a small enough neighborhood of 0 in $\mathcal{H}^{M+4} \subset \mathcal{G}_{0}$ such that

$$
w_{0}=v_{0}+V_{1}\left(v_{0}\right) e^{i x^{2} / 4}+V_{-3}\left(v_{0}\right) e^{-3 i x^{2} / 4} .
$$

LEMMA 4.2.6. - The map $v_{0} \mapsto w_{0}$ given by (4.2.16) is a local diffeomorphism from a neighborhood of 0 in $\mathcal{H}^{M+4}$ to a neighborhood of 0 in $\mathcal{H}^{M+4}$.

Proof. - Since multiplication by $e^{i \lambda x^{2}}$ leaves $\mathcal{H}^{M+4}$ unchanged, we just have to see that $v_{0} \mapsto V_{m}\left(v_{0}\right), m=1,-3$ is bounded from $\mathcal{H}^{M+4}$ to $\mathcal{H}^{M+4}$ and satisfies $\left\|V_{m}\left(v_{0}\right)\right\|_{\mathcal{H}^{M+4}} \leqslant C\left\|v_{0}\right\|_{\mathcal{H}^{M+4}}^{2}$ when $v_{0}$ stays in the unit ball of $\mathcal{H}^{M+4}$. The conclusion will then follow from the local inversion theorem.

Remind the construction of $V_{1}, V_{-3}$ at fixed time $t=1$ : in lemma 4.1.6, we defined symbols $A_{m} m=1,-3$, which are at $t=1$ linear combinations of expressions $a \otimes b$ where $b(\xi)$ is a symbol of order 0 , and $a$ is a quadratic expression of type $a=x^{\gamma_{3}}\left(D_{x}^{\gamma_{1}} v_{0}\right)\left(D_{x}^{\gamma_{2}} v_{0}\right)$ with $\left|\gamma_{1}\right| \leqslant 1,\left|\gamma_{2}\right| \leqslant 1,0<\left|\gamma_{1}\right|+\left|\gamma_{2}\right|+\left|\gamma_{3}\right| \leqslant 2$. One gets 
immediately that $a \in \mathcal{H}^{M+3}$. One then constructs from $A_{m}$ symbols $B_{m}$, through formula (4.1.66), which at $t=1$ gives

$$
B_{m} \in \mathcal{H}^{M+3} \otimes S^{-2}+\mathcal{H}^{M+2} \otimes S^{-3}
$$

and one define $V_{m}\left(v_{0}\right)$ as

$$
V_{m}\left(v_{0}\right)=e^{-i m x^{2} / 4} \mathrm{Op}^{\mathcal{B}}\left(B_{m}\right)\left(e^{i m x^{2} / 4}\right) .
$$

Then proposition 3.2.2, applied at $t=1$, shows that $V_{m} \in \mathcal{H}^{M+4}$. Moreover, since $A_{m}$ is quadratic in $v_{0}$, we have the wanted quadratic estimate. This concludes the proof.

Proof of theorem 1.1.2. - Using the change of unknown (1.2.2), we have to find $v_{\infty}$ such that for any $\gamma \in \mathbb{N}^{2}$ with $|\gamma| \leqslant M$

$$
\left\|x^{\gamma}\left(w(t, x)-v_{\infty}(x)\right)\right\|_{L^{\infty}(d x)}=O\left(t^{-\delta}\right), t \longrightarrow+\infty .
$$

By (4.1.10), $w(t, x)=v(t, x)+V_{1} e^{i \theta}+V_{-3} e^{-3 i \theta}$, where $V_{1}, V_{-3}$ lie in $\mathcal{V} \subset \widetilde{\mathcal{E}}_{\beta, \alpha^{\prime}, 1}^{s+2, s^{\prime}-1}(M)$. We shall choose the indices in (4.1.6) as optimal as possible i.e. $\left(\alpha, \alpha^{\prime}\right)$ close to $(-4,2)$, in the shaded area of the figure of page 64 . Then $s$ will be close to 4 and $\beta=2\left(\alpha+\alpha^{\prime}+1\right)$ will be close to -2 . If $|\gamma| \leqslant M, m=1,-3$, we thus have $x^{\gamma} V_{m} \in \widetilde{E}_{\beta, \alpha^{\prime}, 1}^{s+2, s^{\prime}-1-|\gamma|}$ whence by (3.1.5)

$$
\left\|\chi(D / \sqrt{t}) x^{\gamma} V_{m}\right\|_{L^{\infty}(d x)}=O\left(t^{-\delta}\right), t \longrightarrow+\infty
$$

for any $\delta \in] 0,1 / 2[$. Moreover

$$
\begin{aligned}
\left\|(1-\chi)(D / \sqrt{t}) x^{\gamma} V_{m}\right\|_{L^{\infty}(d x)} \leqslant C \sum_{k ; c \sqrt{t}<2^{k}<t} 2^{k}\left(\frac{2^{k}}{\sqrt{t}}\right)^{-s-2}\left(\frac{2^{k}}{t}\right)^{\alpha^{\prime}} \\
+C \sum_{k ; t<2^{k}} 2^{k}\left(\frac{2^{k}}{\sqrt{t}}\right)^{-s-2}\left(\frac{2^{k}}{t}\right)^{-s^{\prime}+1+|\gamma|}
\end{aligned}
$$

using Sobolev injections and the definition of $E_{\beta, \alpha^{\prime}, 1}^{s+2, s^{\prime}-1-|\gamma|}$. Since $s>\alpha^{\prime}-1, s+s^{\prime}>$ $M \geqslant|\gamma|$, we get an $O\left(t^{-\delta}\right)$ upper bound. Consequently, the asymptotic behaviour of $x^{\gamma} w$ will be given by the one of $x^{\gamma} v$. Since $v \in \underline{\mathcal{E}}_{\alpha, \alpha^{\prime}, \infty}^{s, s^{\prime}}(M)$, we have for $|\gamma| \leqslant M$

$$
\begin{aligned}
\left\|(1-\chi)(D / \sqrt{t}) x^{\gamma} v\right\|_{L^{\infty}(d x)} \leqslant C \sum_{k ; c \sqrt{t}<2^{k}<t} 2^{k}\left(\frac{2^{k}}{\sqrt{t}}\right)^{-s}\left(\frac{2^{k}}{t}\right)^{\alpha^{\prime}} \\
+C \sum_{k ; t<2^{k}} 2^{k}\left(\frac{2^{k}}{\sqrt{t}}\right)^{-s}\left(\frac{2^{k}}{t}\right)^{-s^{\prime}+|\gamma|}
\end{aligned}
$$

and since $s>\alpha^{\prime}+1, s+s^{\prime}>M+1 \geqslant|\gamma|+1$, we get a $O\left(t^{-\delta}\right)$ estimate for this term also. We thus have to study $\chi(D / \sqrt{t})\left(x^{\gamma} v\right)$. Since any term of type $\chi(D / \sqrt{t})\left((D / t) x^{\gamma^{\prime}} v\right)$, 
$\left|\gamma^{\prime}\right| \leqslant|\gamma|-1$ will be also $O\left(t^{-\delta}\right)$ in $L^{\infty}$ (still because $\frac{D}{t}\left(x^{\gamma^{\prime}} v\right) \in \underline{E}_{\alpha+2, \alpha^{\prime}, \infty}^{s, s^{\prime}-\left|\gamma^{\prime}\right|-1}$ ), we reduce ourselves to the study of $\chi(D / \sqrt{t})\left(Z^{\gamma} v\right)$. But it follows from (4.2.8) that

$$
\left|D_{t}\left(\chi(D / \sqrt{t})\left(Z^{\gamma} v\right)\right)\right| \leqslant C t^{-1-\delta}
$$

for any $\delta \in] 0,1 / 2[$ (the fact that $\delta$ can be taken as close as we want to $1 / 2$ follows by inspection of the proof of lemma 4.2.5, using that we took $\left(\alpha, \alpha^{\prime}\right)$ near $\left.(-4,2)\right)$. We deduce from (4.2.18) that $\chi(D / \sqrt{t})\left(Z^{\gamma} v\right)$ has a limit in $L^{\infty}$ when $t \rightarrow+\infty$, and that the distance between the function and its limit is $O\left(t^{-\delta}\right)$. This concludes the proof. 



\section{BIBLIOGRAPHY}

[1] J.-M. Bony - Calcul symbolique et propagation des singularités pour les équations aux dérivées partielles nonlinéaires, Ann. Scient. Éc. Norm. Sup. 14, (1981) 209-256.

[2] A. P. Calderón \& R. Vaillancourt - On the boundedness of pseudodifferential operators, J. Math. Soc. Japan 23, (1971), 374-378.

[3] J.-Y. Chemin - Fluides parfaits incompressibles, Astérisque 230, (1995).

[4] H. Chinara - Local existence for semi-linear Schrödinger equations, Math. Japonica 42, (1995), 35-52.

[5] _ Global existence of small solutions to semi-linear Schrödinger equations, Comm. Partial Differential Equations 21, (1996), 63-78.

[6] - The initial value problem for cubic semi-linear Schrödinger equations, Publ. RIMS, Kyoto Univ. 32, (1996), 445-471.

[7] S. CoHN - Global existence for the nonresonant Schrödinger equation in two space dimensions, Canad. Appl. Math. Quart. 2, (1994), 247-282.

[8] J.-M. DELORT - Existence globale et comportement asymptotique pour l'équation de Klein-Gordon quasi linéaire à données petites en dimension 1, Ann. Scient. Éc. Norm. Sup. 34, (2001), 1-61.

[9] S. DoI - On the Cauchy problem for Schrödinger type equations and the regularity of the solutions, J. Math. Kyoto Univ. 34, (1994), 319-328.

[10] N. HayAshi \& H. HiRATA - Global existence of small solutions to nonlinear Schrödinger equations, Nonlinear Analysis, Theory, Methods and Applications 31, (1998), 671-685.

[11] N. HAYASHI \& K. KATO - Global existence of small analytic solutions to Schrödinger equations with quadratic nonlinearities, Comm. Partial Differential Equations 22, (1997), 773-798. 
[12] N. Hayashi, C. Miao \& P.I. NAUMkin - Global existence of small solutions to the generalized derivative nonlinear Schrödinger equation, Asymptotic Analysis 21, (1999), 133-147.

[13] N. HAYASHI \& P.I. NAUMKIN - On the quadratic nonlinear Schrödinger equation in three space dimensions, Internat. Math. Res. Notices (2000), 115-132.

[14] _ Asymptotics of small solutions to nonlinear Schrödinger equations with cubic nonlinearities, preprint, $12 \mathrm{pp}$.

[15] _ Global existence of small solutions to the quadratic nonlinear Schrödinger equation in two space dimensions, SIAM J. Math. Anal. 32 (2001), no. 6, 13901409 .

[16] _ Asymptotic expansion of small analytic solutions to the quadratic nonlinear Schrödinger equation in two space dimensions, preprint, $15 \mathrm{pp}$.

[17] _ A quadratic nonlinear Schrödinger equation in one space dimension, preprint, $18 \mathrm{pp}$.

[18] N. Hayashi \& T. OzaWA - Remarks on nonlinear Schrödinger equations in one space dimension, Diff. Integral Eqs 7, (1994), 453-461.

[19] C. Kenig, G. Ponce \& L. VeGA - Small solutions to nonlinear Schrödinger equations, Ann. Inst. Henri Poincaré 10, (1993), 255-288.

[20] S Smoothing effects and local existence theory for the generalized nonlinear Schrödinger equations, Invent. Math. 134 (1998), no. 3, 489-545. 